\title{
DYNAMICAL SYSTEMS COUPLED WITH MONOTONE SET-VALUED OPERATORS: FORMALISMS, APPLICATIONS, WELL-POSEDNESS, AND STABILITY*
}

\author{
BERNARD BROGLIATO $^{\dagger}$ AND ANEEL TANWANI ${ }^{\ddagger}$
}

\begin{abstract}
This survey article addresses the class of continuous-time systems where a system modeled by ordinary differential equations (ODEs) is coupled with a static and time-varying setvalued operator in the feedback. Interconnections of this form model certain classes of nonsmooth systems including sweeping processes, differential inclusions with maximal monotone right-hand side, complementarity systems, differential and evolution variational inequalities, projected dynamical systems, some piecewise linear switching systems. Such mathematical models have seen applications in electrical circuits, mechanical systems, hysteresis effects, and many more. When we impose a passivity assumption on the open-loop system, and regard the set-valued operator in the feedback as maximally monotone, we obtain a set-valued Lur'e dynamical system. In this article we review the mathematical formalisms, their relationships, main application fields, well-posedness (existence, uniqueness, continuous dependence of solutions), and stability of equilibria. An exhaustive bibliography is provided.
\end{abstract}

Key words. Lur'e systems, set-valued systems, passivity, well-posedness, differential inclusions, normal cones, tangent cones, maximal monotone operators, prox-regular sets, Moreau's sweeping process, complementarity problems, complementarity systems, projected dynamical systems, piecewise linear systems, Filippov's differential inclusions, Lyapunov stability, absolute stability, Lagrangian systems, circuits.

AMS subject classifications. 34A36, 34A60, 49K21, 49J52, 49J53, 93D15, 93D20.

\section{Contents}

1 Introduction

2 Modeling frameworks $\quad 5$

2.1 Moreau's sweeping processes $(\mathrm{SwP}) \ldots \ldots \ldots \ldots \ldots$

2.2 Maximal monotone differential inclusions . . . . . . . . . . . . . . 15

2.3 Differential variational inequalities (DVI) $\ldots \ldots \ldots \ldots \ldots$

2.4 Complementarity systems . . . . . . . . . . . . . . . . . . . . . . 19

2.5 Projected dynamical systems (PDS) $\ldots \ldots \ldots \ldots \ldots \ldots$

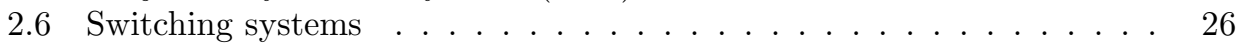

3 Relationships between various formalisms $\quad 29$

3.1 Maximal monotone DIs, complementarity systems, FOSwP, and DVIs 30

3.2 FOSwP, SOSwP, EVIs and QVIs . . . . . . . . . . . . . . . . 31

3.3 FOSwP and compact DIs . . . . . . . . . . . . . . . . . . 32

3.4 Complementarity systems, FOSwP, relay systems and maximal mono-

tone DIs . . . . . . . . . . . . . . . . . 32

3.5 ZOSwP, complementarity systems, projected systems . . . . . . . . 35

3.6 Degenerate FOSwP, complementarity systems . . . . . . . . . . . 35

3.7 SOSwP and complementarity systems . . . . . . . . . . . 36

*Submitted to the editors DATE. This work is partially supported by the ANR project CONVAN with grant number ANR-17-CE40-0019.

${ }^{\dagger}$ Univ. Grenoble Alpes, Inria, CNRS, Grenoble INP, LJK, 38000 Grenoble, France. (bernard.brogliato@inria.fr).

${ }^{\ddagger}$ LAAS - CNRS, 7 Avenue du Colonel Roche, 31400 Toulouse, France. (aneel.tanwani@laas.fr). 
3.8 Projected dynamical systems, DIs and complementarity systems . . . 36

3.9 HOSwP, complementarity systems, VIs . . . . . . . . . . . . . . 38

3.10 Relay systems and LCS . . . . . . . . . . . . . . . . . . 38

3.11 PWL systems and complementarity systems . . . . . . . . . . . . . 39

3.12 Complementarity systems and nonsmooth DAEs . . . . . . . . . 43

3.13 Maximal monotone DIs and convex processes . . . . . . . . . . . . 43

3.14 Mechanical systems with impacts and Filippov's DIs . . . . . . . . . . 44

3.15 Hysteresis operators, DIs, complementarity systems, FOSwP . . . . . 44

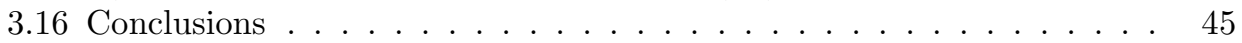

4 Applications $\quad 46$

4.1 Electrical circuits . . . . . . . . . . . . . . . . . . . 46

4.2 Multibody mechanical systems with unilateral contact . . . . . . . . 49

4.3 Other Applications . . . . . . . . . . . . . . . 56

5 Well-posedness results $\quad 58$

5.1 Regularization of FOSwP . . . . . . . . . . . . . . . . . . 59

5.2 Discretization via Time-Stepping Algorithm . . . . . . . . . . . . 63

5.3 Maximal monotone DIs . . . . . . . . . . . . . . . . . 69

5.4 Solutions of SOSwP . . . . . . . . . . . . . . . . 72

5.5 Higher order sweeping processes . . . . . . . . . . . . . . . 74

5.6 Extensions and Perspectives . . . . . . . . . . . . . 76

6 Stability of equilibria $\quad \mathbf{7 7}$

6.1 Characterization of equilibria . . . . . . . . . . . . . 77

6.2 Sufficient Lyapunov Conditions . . . . . . . . . . . . . . . . . . 80

6.3 Krasovskii-LaSalle invariance principle . . . . . . . . . . . . . . 84

6.4 Passivity and absolute stability . . . . . . . . . . . . . . . 86

$\begin{array}{llr}7 & \text { Conclusions } & 88\end{array}$

$\begin{array}{lr}\text { Appendices } & 89\end{array}$

$\begin{array}{ll}\text { A Some tools from convex analysis } & 89\end{array}$

$\begin{array}{ll}\text { B Some tools from complementarity theory } & 98\end{array}$

$\begin{array}{lr}\text { C Relative degree } & 99\end{array}$

$\begin{array}{lr}\text { D Dissipative systems } & 100\end{array}$

$\begin{array}{ll}\text { E Basic definitions, notation, and results } & 101\end{array}$

$\begin{array}{lr}\text { References } & 102\end{array}$ 
1. Introduction. The central object of this survey article is a class of dynamical systems where a system of nonlinear differential equations is interconnected with setvalued mappings as depicted in Figure 1.1. The resulting object, in general, is a differential inclusion of the form:

$$
\left\{\begin{array}{l}
\dot{x}=f(t, x)+G(t, x) w \\
w \in-\mathcal{F}(t, H x, J w) \\
x(0)=x_{0},
\end{array}\right.
$$

where $x: \mathbb{R}_{+} \rightarrow \mathbb{R}^{n}$ is the state trajectory, $f: I \times \mathbb{R}^{n} \rightarrow \mathbb{R}^{n}$ and $G: I \times \mathbb{R}^{n} \rightarrow \mathbb{R}^{n \times m}$ are single-valued, $H$ and $J$ are constant matrices of appropriate dimensions. In (1.1), $w \in \mathbb{R}^{m}$ is a solution of the inclusion $w+\mathcal{F}(t, H x, J w) \ni 0$, where $\mathcal{F}(t, H x, J w)$ is a set-valued function and in Section 2, it will be shown that the multivalued mapping $\mathcal{F}$ can take different forms and that, $w$ can be obtained as a solution to an underlying optimization problem. The framework of (1.1) originates from the study of Lur'e systems [401], where a linear time-invariant system is coupled with a static nonlinearity in the feedback loop. The absolute stability problem, studied in the context of such systems, relates to analyzing a class of nonlinearities such that the resulting coupled system is globally asymptotically stable. It has been extensively used for feedback control problems, see [392] for a survey. Studying the stability of such interconnections when the feedback loop is a "relation" (i.e., something more general than a single-valued nonlinearity) was advocated in [573, 572, 574, 581, 582]. Imposing certain sector bounds on the static nonlinearity, and requiring the (open-loop) linear system to be passive (or the transfer matrix to be positive real), allows us to formulate constructive stability conditions.

The approach adopted in this article for analyzing the differential inclusion (1.1) is somewhat inspired by this underlying philosophy behind Lur'e systems [401, 575], so that seeing the evolution equation as a sum of a vector field and set-valued mapping with nice enough properties, makes the interconnection amenable for analysis. The ease of analysis is not the only reason for opting the framework (1.1) as the central object; it is also their broad applicability across various domains of applied mathematics and engineering. As we will see, different mathematical models for evolution processes can be written out in this form. These processes appear in numerous applications ranging from modeling physical phenomenon to synthesizing control laws which are discontinuous or set-valued mappings of the state.

Our interest in writing this article mainly lies in studying the nonsmooth phenomenon that arises in these models, which of course is motivated either by physical phenomenon or by engineering control systems. In particular, the maximal monotonicity

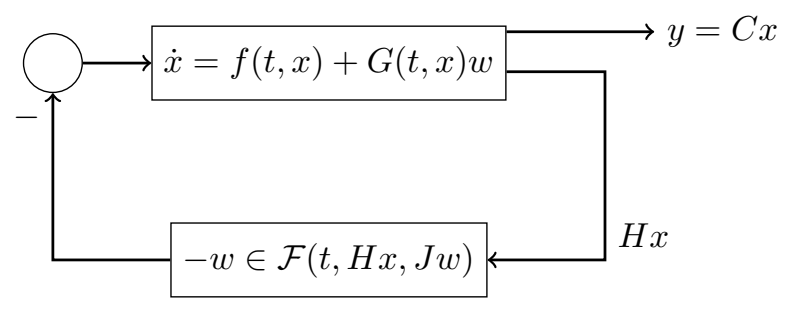

Fig. 1.1: Dynamical systems with multivalued nonlinearities in feedback. 
of multivalued mappings and the convexity of sets (or notions which are close to these ones in a certain sense), are central properties, as well as the passivity of the vector field $f(t, x)$ for stability purposes. This is the reason why not all differential inclusions will be, by far, considered in this article, but only specific classes. This article deals with finite-dimensional systems, hence the state space is $\mathbb{R}^{n}$. Clearly extensions to infinite-dimensional systems and Hilbert or Banach spaces are worth investigating and will usually constitute a qualitative "jump" in the analysis. However there is another important qualitative "jump" on which we shall focus: relaxing convexity. When nonsmooth dynamical systems are the subject, making such a step is not obvious.

The way this article is organized is as follows: In Section 2, we visit different classes of differential inclusions which can be modeled within the framework of (1.1). These include models ranging from sweeping processes of different order, maximal monotone differential inclusions, evolution inequalities, complementarity systems, projected dynamical systems, and more related formalisms. In Section 3, we study the equivalences between these models in an attempt to draw connections between approaches that exist for analyzing them. The applications of these models are then studied in Section 4 . We focus our attention on applications in electrical circuits and mechanical systems (with and without friction), and also provide a list of other applications with references which have been studied using the frameworks described in Section 2. Then, in Section 5, we study the tools developed in the literature for proving the existence of solutions of such systems, and in particular, we visit in detail the methods based on constructing discretized solutions and the analysis carried out in proving the convergence (with respect to the time-step) of these solutions. Lastly, in Section 6, we study the stability of the dynamical systems from the control-theoretic perspective. We highlight the interesting aspects that arise in stability of nonsmooth dynamical and provide a brief account of the generalization of conventional control-theoretic methods to set-valued systems. Useful mathematical tools from convex and nonsmooth analysis, complementarity theory, dissipative systems, are provided in the appendices.

\section{Notation and Abbreviations.}

$\mathcal{N}_{K}(x)$

$\mathcal{T}_{K}(x)$

$\mathcal{T}_{K}^{h}(x)$

$\partial \varphi$

$\psi^{*}$

$\psi_{\mathcal{S}}$

$\sigma_{\mathcal{S}}$

$d_{\mathcal{S}}(x)$

$d_{\text {Haus }}\left(\mathcal{S}_{1}, \mathcal{S}_{2}\right)$

$K^{\circ}$

$K^{\star}$

$V(q)$

$\operatorname{proj}[K ; x]$

$\mathrm{AC}$

D/EVI

DAE

DI

FOSwP

HOSwP

LCCP/LCCS
Normal cone to the set $K$ at point $x$

Tangent cone to the set $K$ at point $x$

Tangent linearization cone to the set $K$ at point $x$

Subdifferential of the function $\varphi$

Convex conjugate of the function $\psi$

Indicator function of the set $\mathcal{S}$

support function of the set $\mathcal{S}$

Euclidean distance of $x$ from the set $\mathcal{S}$, i.e., $d_{\mathcal{S}}(x)=\inf _{y \in \mathcal{S}}|x-y|$

Hausdorff distance between the sets $\mathcal{S}_{1}$ and $\mathcal{S}_{2}$

The polar cone of the set $K$

The dual cone of the set $K$

Velocity cone $\mathcal{T}_{\Phi}^{h}(q)$ at $q \in \Phi$

Orthogonal projection of $x$ on the set $K$

Absolutely continuous

Differential/Evolution variational inequality

Differential-algebraic equation

Differential inclusion

First order sweeping process

Higher order sweeping process

Linear cone complementarity problem/system 


$\begin{array}{ll}\text { LCP/LCS } & \text { Linear complementarity problem/system } \\ \text { LSC } & \text { Lower semicontinuous } \\ \text { MDI } & \text { Measure differential inclusion } \\ \text { MFCQ } & \text { Mangasarian-Fromovitz constraint qualification } \\ \text { MLCS } & \text { Mixed linear complementarity system } \\ \text { NLCS } & \text { Nonlinear complementarity system } \\ \text { PDS } & \text { Projected dynamical systems } \\ \text { PWL } & \text { Piecewise linear } \\ \text { RC(L)BV } & \text { Right-continuous locally of bounded variation } \\ \text { SOSwP } & \text { Second order sweeping process }\end{array}$

In addition, we use the following conventional notation: $\operatorname{bd}(\mathcal{S})$ for boundary of the set $\mathcal{S} ; \operatorname{int}(\mathcal{S})$ for interior of the set $\mathcal{S} ; \operatorname{rint}(\mathcal{S})$ for relative interior of the set $\mathcal{S} ; \operatorname{cl}(\mathcal{S})$ for the closure of the set $\mathcal{S}$; $\operatorname{dom}(f)$ and $\operatorname{im}(f)$ for the domain and range, respectively, of a single-valued or set-valued function $f ; \mathbb{B}_{r}^{n}(x)$ for the closed ball of radius $r$ in $\mathbb{R}^{n}$ centered at $x ; P \succ 0$ (or, $P \succcurlyeq 0$ ) to denote that the matrix $P$ is positive definite (or, positive semi-definite), not necessarily symmetric; $\mathcal{C}^{0}\left([0, T], \mathbb{R}^{n}\right)$ for the space of continuous functions from $[0, T]$ to $\mathbb{R}^{n}$ equipped with maximum norm; $\mathcal{L}^{p}\left([0, T], \mathbb{R}^{n}, d \nu\right)$ for the space of functions $f(\cdot)$ with $\int_{[0, T]}|f|^{p} d \nu<\infty$. For a matrix $A \in \mathbb{R}^{n \times m}, A_{i \bullet}$ is its $i$ th row, $A_{\bullet} i$ is its $i$ th column, $A_{\mathcal{I}} \bullet$ with $\mathcal{I} \subseteq\{1, \ldots, n\}$ is the submatrix made of rows indexed in $\mathcal{I}, A_{\bullet} \mathcal{I}$ is the submatrix made of columns indexed in $\mathcal{I}$. The reader may consult Appendix A and Appendix E for definitions and further clarifications.

2. Modeling frameworks. In this section, we take the first step in interpreting the utility of the system class proposed in (1.1). This is done by recalling the mathematical models of different classes of nonsmooth set-valued systems and recasting them in the form of (1.1).

2.1. Moreau's sweeping processes (SwP). The so-called system class of sweeping processes was introduced by Jean Jacques Moreau in [434, 435, 436, 439]. The basic idea of these processes is to describe the movement of a point contained inside a set, and as the set moves with time, the point is swept by it. The mathematical model for describing this motion consists of a family of time-varying DIs and comprises several subclasses which can be defined from the "order" of the sweeping process, depending on how the underlying set is parameterized. In the remainder of Section 2.1, we describe the basic structure of these different sweeping processes, and a glimpse of how the analysis gets more complicated as the order increases.

2.1.1. First order sweeping process (FOSwP). The simplest class of sweeping processes proposed in $[434,435,436,439]$ are now more commonly referred as those of first order. In the simplest setting, we consider a time-varying set-valued mapping $\mathcal{S}: \mathbb{R}_{+} \rightarrow \mathbb{R}^{n}$, such that $\mathcal{S}(t) \subseteq \mathbb{R}^{n}$ is a closed non empty and convex set for each $t \geq 0$. We also assume some regularity for the set-valued mapping $\mathcal{S}$ with respect to time in the sense that, there exists an AC function $\mu: \mathbb{R}_{+} \rightarrow \mathbb{R}$ such that

$$
d_{\text {Haus }}\left(\mathcal{S}\left(t_{1}\right), \mathcal{S}\left(t_{2}\right)\right) \leq\left|\mu\left(t_{1}\right)-\mu\left(t_{2}\right)\right|,
$$

where $d_{\text {Haus }}\left(\mathcal{S}_{1}, \mathcal{S}_{2}\right)$ denotes the Hausdorff distance between the sets $\mathcal{S}_{1}$ and $\mathcal{S}_{2}$. The FOSwP (also called the perturbed sweeping process [237, 238]) corresponds to finding a function $x: \mathbb{R}_{+} \rightarrow \mathbb{R}^{n}$ which satisfies the following set of inclusions:

$$
\left\{\begin{array}{l}
x(t) \in \mathcal{S}(t), \text { for all } t \geq 0 \\
\dot{x}-f(t, x) \in-\mathcal{N}_{\mathcal{S}(t)}(x),
\end{array}\right.
$$


for a given time-varying vector field $f: \mathbb{R}_{+} \times \mathbb{R}^{n} \rightarrow \mathbb{R}^{n}$ (originally Moreau studied unperturbed FOSwP with $f(t, x) \equiv 0)$. Under the stated assumptions on $\mathcal{S}(t)$, the normal cone defines a maximal monotone mapping for each fixed $t$. A solution of (2.2) corresponds to finding a function $x$, and a selection $\eta: \mathbb{R}_{\geq 0} \rightarrow \mathbb{R}^{n}$ such that $\eta(t) \in \mathcal{N}_{\mathcal{S}(t)}(x(t))$ and $\dot{x}(t)-f(t, x(t))=-\eta(t)$ holds for Lebesgue almost every $t \geq 0$. Roughly speaking, the meaning of $(2.2)$ is that for each $t \geq 0$, if $x(t) \in \operatorname{int}(\mathcal{S}(t))$ then $\mathcal{N}_{\mathcal{S}(t)}(x(t))=\{0\}$ and $(2.2 \mathrm{~b})$ becomes an ODE $\dot{x}=f(t, x)$. When $x(t) \in \operatorname{bd}(\mathcal{S}(t))$, and $\dot{x}(t)$ points outside $\mathcal{S}(t)$, it is possible to choose $\eta\left(t^{+}\right)$that points strictly inside the set $\mathcal{S}(t)$ and corrects the vector field so that $x(\cdot)$ does not violate the constraint (2.2a), see Figure 2.1a. This is closely related to viability [62], and such systems are sometimes called systems with reflection at the boundary $[505,506]$.

REMARK 1. To simplify the presentation, in the sequel the normal and tangent cones to a convex set $K$ at $x$, will be denoted generically as $\mathcal{N}_{K}(x)$ and $\mathcal{T}_{K}(x)$ without further details, except when it is necessary. See Appendix A for a summary on various useful tools from Convex and Nonsmooth Analysis, in particular section A.1 for normal and tangent cones. Also, it is understood that when solutions are $A C$, then equalities or inclusions for the derivatives are satisfied Lebesgue almost everywhere. This will not be repeated often to lighten the presentation (thus avoiding $\stackrel{\text { a.e. }}{=}, \stackrel{\text { a.e. }}{\in}, \stackrel{\text { a.e. }}{\geq}$, etc).

It is noted that under the absolute continuity condition on the mapping $\mathcal{S}(\cdot)$ and the fact that $x(0) \in \mathcal{S}(0)$, the resulting solution $x(\cdot)$ is an AC function. In case, the regularity on $\mathcal{S}$ is relaxed in the sense that (2.1) holds with right-hand side replaced by $\left.d \mu(] t_{1}, t_{2}\right]$ ), with $\mu$ being locally RCBV (see Appendix E), then it is still possible to reformulate the FOSwP given in (2.2). In this case, we call a locally RCBV function $x: \mathbb{R}_{+} \rightarrow \mathbb{R}^{n}$ a solution to FOSwP if there exists a positive measure $d \nu$ (AC with respect to $d t+d \mu$ ), relative to which the Stieltjes (or differential) measure $d x$ possesses a density $\dot{x}$, i.e., $d x=\dot{x}(t) d \nu$, and $(2.2 \mathrm{~b})$ is replaced by the Measure Differential Inclusion (MDI)

$$
\frac{d x}{d \nu}(t)-f(t, x) \frac{d t}{d \nu}(t)=\eta(t), \quad \nu \text { a.e. } t \geq 0,
$$

in which $\eta(t)$ takes values in $\mathcal{N}_{\mathcal{S}(t)}(x(t))$. In the $\mathrm{RCBV}$ case, $x(\cdot)$ may jump at

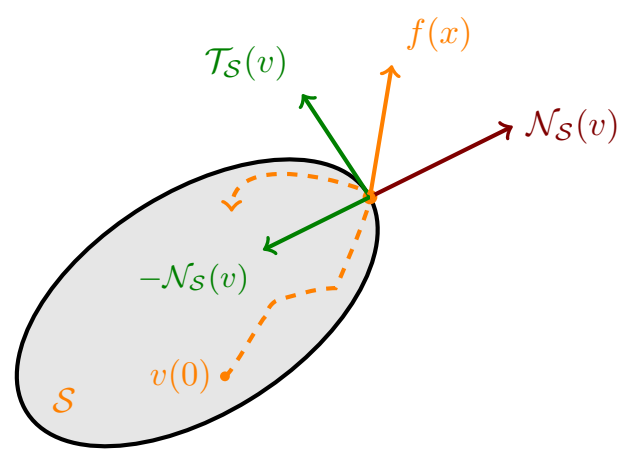

(a) Vector fields of interest in FOSwP.

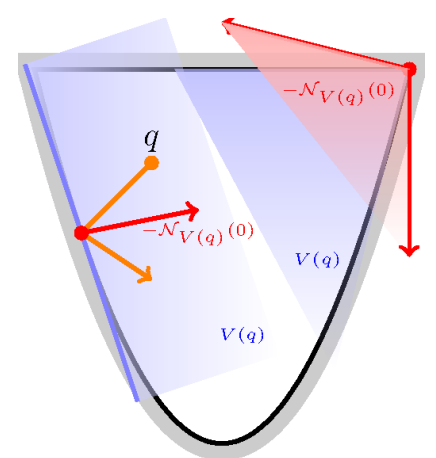

(b) A SOSwP with velocity jump.

Fig. 2.1: Graphical description of first and second order sweeping processes. 
countably many time instants and $\nu$ has atoms at discontinuity times of $\nu[439,427$, $27,238]$. The MDI (2.3) can be rewritten compactly and equivalently as the measure inclusion

$$
d x-f(t, x) d t \in-\mathcal{N}_{\mathcal{S}(t)}(x)
$$

by choosing $\nu=\mu+d t$ in (2.3) [238], where $d x$ is the differential, or Stieltjes measure associated with the BV function $x(\cdot)$ (see Appendix $\mathrm{E}$ and $[427,6]$ for a rigorous definition of the inclusion of a measure in a convex cone, and [522, section 4.1] for the interpretation of (2.3)). Comparing with the formulation presented in (1.1), we have

$$
w=\eta, \quad \mathcal{F}(t, H x, J w)=\mathcal{N}_{\mathcal{S}(t)}(x), \quad G(t, x)=I_{n} .
$$

$\rightsquigarrow A$ strong feature of the FOSwP and all its variants, is that the solutions, when they exist, are continuous provided the set $\mathcal{S}(t)$ is continuous and the initial conditions are properly chosen. In other words, jumps in the state $x(\cdot)$ can only be forced through exogenous actions that make $\mathcal{S}(\cdot)$ discontinuous, or with specific initial data. This is in big contrast with the SOSwP and HOSwP of the next sections where state jumps are autonomous and state-triggered. Another feature is the underlying complementarity between two variables, depending on whether $x(t) \in \mathrm{bd}(\mathcal{S}(t)$ ) (then one may have $\eta(t) \neq 0$ ), or $x(t) \in \operatorname{int}(\mathcal{S}(t)$ ) (in this case it follows that $\eta(t)=0$ since one has $\left.\mathcal{N}_{\mathcal{S}(t)}(x(t))=\{0\}\right)$. More details are given in section 3.4.

Generalisations: Many extensions of the basic FOSwP have been studied since the original work of Moreau [512,372]. Let us propose a short summary with the main directions. The basic FOSwP could a prori be generalized in several directions: from finite to infinite dimensions in Hilbert or Banach spaces, from convex to various kinds of nonconvex sets $\mathcal{S}(t)$ (with variation described by AC, BV, or stochastic functions), from time-varying $\mathcal{S}(t)$ to state-dependent (or state and velocity dependent) $\mathcal{S}(t, x)$, with single-valued or set-valued perturbations $f(t, x)$, and from the relative degree -or order- one to higher orders (this last extension will be made clear later). Let us describe few of these extensions such that the system remains in the FOSwP framework. Notice that the following results focus mainly on existence and uniqueness of solutions.

1. (Degenerate FOSwP) If, instead of $x$, the vector $H x$ is constrained by the set $\mathcal{S}(t)$, for some operator $H$ (possibly set-valued, Lipschitz continuous and maximal strongly monotone), then we work with $\mathcal{N}_{\mathcal{S}(t)}(H x)$ in $(2.2 \mathrm{~b})$, a setup which is referred to as degenerate FOSwP $[25,369,371,522]$. For instance we may have $H(x)=\partial \varphi(x)$ for some LSC convex proper function $\varphi(\cdot)$ (including the case $H=H^{T} \succcurlyeq 0$ if $H \in \mathbb{R}^{n \times n}$ is a constant matrix), the difficulty here is that the composition of two monotone operators is not monotone in general. The case with $\mathcal{N}_{\mathcal{S}(t)}(H x+G \eta)$ is analysed in [524] and could be seen as an extension of the basic degenerate FOSwP (see Section 3.4 for more comments).

2. (Non-convex $\mathcal{S}(t)$ ) The first issue with non convex $\mathcal{S}(\cdot)$, concerns the choice of the normal cone. Consider for instance the set $K_{3}$ in Example 10, Section A.1.2. Clearly changing the normal cone definition will modify significantly the obtained DI (in this particular case, Clarke's normal cone is too big to be of any interest, while Fréchet's cone looks more appropriate). The first nonconvex FOSwP were analysed in $[543,544,545,169]$, with $\mathcal{S}(t)=\mathbb{R}^{n} \backslash \operatorname{int}(K(t))$, and $K(t) \subseteq \mathbb{R}^{n}$ convex (or just Lispchitzean multifunction [169]) set with nonempty interior. Clarke's normal cone was used. The well-posedness is studied in $[42,38,143$, $204,238,241,293,530,117,444,446,504]$ assuming that $\mathcal{S}(t)$ is uniformly 
prox-regular for each $t \geq 0$ (hence any of the normal cones $\mathcal{N}_{\mathcal{S}(t)}^{p}(x), \widehat{\mathcal{N}}_{\mathcal{S}(t)}(x)$, $\mathcal{N}_{\mathcal{S}(t)}^{s}(x)$, or $\mathcal{N}_{\mathcal{S}(t)}^{C}(x)$ can be considered, see Section A.1.2). Prox-regularity is a property of interest in nonconvex setup that is amenable for stability analysis $[522,562]$, because, loosely speaking, it possesses some kind of local convexity and normal cones are locally hypomonotone (see Definition A.2 (iii) (iv)). The perturbation $f(t, x)$ takes the form of a maximal monotone set-valued operator $A(x)$ in [38], hence modifying $\mathcal{F}(t, x)=A(x)+\mathcal{N}_{\mathcal{S}(t)}(x)$, and it takes the form $\partial V(x)+U(t, x)$ for some convex bounded function $V(\cdot)$ and multivalued mapping $U(\cdot, \cdot)$ with closed values, in $[535,539]$, with polyhedral $\mathcal{S}(t)$ (a case also analysed in [202] without perturbation). The possibility of Brownian $\mathcal{S}(\cdot)$ with unbounded variation, is pointed out in [204, Example 1]. Hausdorff-Lipschitzean set functions $\mathcal{S}(\cdot)$ may be considered [99], as well as positively $\alpha$-far sets [295, 296, 338], with an upper semicontinuous set-valued perturbation and state-dependent $\mathcal{S}(t, x)$ in [296]. The FOSwP with $f(t, x) \equiv 0$ is shown to be equivalent to a time-varying unconstrained DI in [295], the time-variation stemming from the variation rate of the $\mathrm{AC}$ set $\mathcal{S}(t)$.

3. (State-dependent $\mathcal{S}(t, x)$ ) The first contribution dealing with $\mathcal{S}(t, x)$ is in [370], followed by $[37,170,293,27,339,293,67,186,340]$, degenerate FOSwP with prox-regular sets are studied in [25] in this framework. Convex compact Lipschitzean sets $\mathcal{S}(t, x)$ are considered in [186]. In [339] the set $\mathcal{S}(t, x)$ is supposed uniformly subsmooth, and has Lipschitz-like and compactness properties (the Clarke normal cone is considered), [37, 376] assume Lipschitzean sets, while in $[67,186,293], \mathcal{S}(t, x)$ is assumed to be prox-regular and to vary in an AC way. A fundamental assumption in state-dependent FOSwP is that $\mathcal{S}(t, x)$ has to be Lipschitz continuous in both variables in the Hausdorff distance, with the $x$ Lipschitz constant $L_{x}$ satisfying $0 \leq L_{x}<1$. As proved in [370] this is a necessary condition for existence of solutions (this stems from the fact that $x \in \mathcal{S}(t, x)$ for all $t>0$ which may not hold if $L_{x} \geq 1$ [370, Example 3.6]).

4. (Generalized FOSwP) The so-called generalized FOSwP is introduced in [338] and takes the form: $\dot{x} \in-g(x) \mathcal{N}_{\mathcal{S}(t)}(h(x))+F(t, x), x(0) \in h^{-1}(\mathcal{S}(0))$, where $g(\cdot)$ is a single-valued continuous function satisfying a linear growth condition, $h(\cdot)$ is differentiable with bounded Jacobian, and $\frac{\partial h}{\partial x} g(x) \succ 0$. Particular forms of generalized FOSwP have been studied also in [143, Section 4], with different assumptions.

5. (Retarded FOSwP) FOSwP with time-delays in the perturbation $f(t, x)$ are studied in $[177,117]$ (compact prox-regular $\mathcal{S}(t)$ ), [294] (prox-regular $\mathcal{S}(t, x)$ ), [174] $(\mathrm{AC}$ and Lipschitz $\mathcal{S}(t))[236,235]$ (AC and prox-regular $\mathcal{S}(t)$ ).

6. (Zero Order SwP) Another variant of the FOSwP may be found in [114]: $\dot{x} \in$ $-\mathcal{N}_{\mathcal{S}(t)}(\dot{x})$ ( $\Leftrightarrow \dot{x}=\operatorname{proj}[\mathcal{S}(t) ; 0]$ from (A.6) in case $\mathcal{S}(t)$ is closed convex), and: $x \in-\mathcal{N}_{\mathcal{S}(t)}(\dot{x})\left(\Leftrightarrow \dot{x} \in \partial \sigma_{\mathcal{S}(t)}(x)\right.$, see Section A.2.4 for this equivalence in case $\mathcal{S}(t)$ is closed convex). This was extended in [27], with a FOSwP that takes the implicit form (in finite dimensional setting): $A_{1} \dot{x}+A_{0} x+F(t) \in-\mathcal{N}_{\mathcal{S}(t)}(\dot{x})$ for some matrices $A_{1}=A_{1}^{T} \succcurlyeq 0$ and $A_{0}=A_{0}^{T} \succcurlyeq 0$, and $\mathcal{S}(t)$ bounded for all $t$ (hence $\mathcal{N}_{\mathcal{S}(t)}(\cdot)$ is surjective). We see that this DI can be rewritten equivalently as: $\dot{x} \in$ $\left(A_{1}+\mathcal{N}_{\mathcal{S}(t)}\right)^{-1}\left(-A_{0} x-F(t)\right)$, so that its analysis relies heavily on the properties of the time-varying operator $\left(A_{1}+\mathcal{N}_{\mathcal{S}(t)}\right)^{-1}(\cdot)$ (conditions under which this is a single-valued operator for each $t$ even with $A_{1} \succcurlyeq 0$ are studied in [135] in the finite-dimensional case). In particular the surjectivity of $\mathcal{N}_{\mathcal{S}(t)}(\cdot)$, guaranteed by the boundedness of $\mathcal{S}(t)$, allows to choose arbitrary initial condition $x(0)$, because 
$\operatorname{Im}\left(A_{1}+\mathcal{N}_{\mathcal{S}(t)}\right)=\operatorname{dom}\left(\left(A_{1}+\mathcal{N}_{\mathcal{S}(t)}\right)^{-1}\right)=\mathbb{R}^{n}$. The boundedness of $\mathcal{S}(t)$ is relaxed in [39], where the surjectivity of the operator $x \mapsto A_{1} x+\mathcal{N}_{\mathcal{S}(t)}(x)$ is guaranteed by other means. The case of state-dependent closed convex Lipschitzean set $\mathcal{S}(t, x)$ is analysed in [24] (contrarily to the state-dependent FOSwP, the Lipschitz constant $L_{x}$ needs not satisfy $L_{x}<1$ ). In [23] a further variant of the FOSwP: $\dot{x} \in-\mathcal{N}_{\mathcal{S}(t)}(A \dot{x}+B x)$ is analysed, with $A=A^{T} \succ 0$ and $B=B^{T} \succcurlyeq 0$ (in the finite-dimensional setting), $\mathcal{S}(\cdot)$ closed convex for each $t$ and AC. This can be rewritten equivalently as $\dot{x} \in-\left(A+\mathcal{N}_{\mathcal{S}(t)}^{-1}\right)^{-1} B x$, hence this time the analysis relies on the properties of the operator $\left(A+\mathcal{N}_{\mathcal{S}(t)}^{-1}\right)^{-1}$, where we recall that $\mathcal{N}_{\mathcal{S}(t)}^{-1}=$ $\partial \sigma_{\mathcal{S}(t)}$ (see Section A.2.4). This is extended in [26] with state dependent $\mathcal{S}(t, x)$, and in [341] who considers nonlinear perturbation $f(t, x)$ and $A \dot{x}+h(t, x)$ [341, Theorem 5.1]. Roughly speaking, [23, 341] and [27] treat inverse problems, where the normal cone is replaced by the support function. These last two systems may be named Zero Order Sweeping Processes $(\mathrm{ZOSwP})$, for a reason that will be made clear in Section 3.5. In [65], the normal cone operator is replaced by a general operator $A(t)(\cdot)$ that is maximal monotone for each $t$.

7. (Stochastic SwP) Stochastic FOSwP has been studied in [166, 167] (and also in the framework of the stochastic Skorokhod problem), and more recently in $[175,176,204,103,225]$, where the stochasticity appears in the perturbation $f(\cdot)$ $[103,175,176]$ or in a prox-regular set $\mathcal{S}(\cdot)[204$, Section 5].

8. (Perturbation) Classical FOSwP involves single-valued perturbations. However many articles consider set-valued perturbations $f(t, x)[67,65,64,169,241,296$, $338,444,446,535]$ (initiated in [425], see also [169] for references to the Séminaire d'Analyse Convexe de Montpellier), integral perturbations are studied in [203, 119].

2.1.2. Second order sweeping process (SOSwP). Building up on the formalism given in (2.2) for modeling trajectories with constraints, J.J. Moreau also introduced an extension in the form of SOSwP in [441, 442] to model Lagrangian systems with unilateral constraints and impacts which induce velocity discontinuities. We shall come back on Lagrangian systems in Section 4.2.1. However in its simplest form (constant unit mass matrix), the SOSwP, with position variable $q \in \mathbb{R}^{\frac{n}{2}}$, and the velocity variable $v \in \mathbb{R}^{\frac{n}{2}}$, is described as:

$$
\left\{\begin{array}{l}
q(t) \in \Phi, v(t) \in V(q(t)) \text { for all } t \geq 0 \\
\dot{q}=v \text { Lebesgue almost everywhere } \\
d v-F(t, q, v) d t \in-\mathcal{N}_{V(q)}\left(v_{e}(t)\right)
\end{array}\right.
$$

where $v_{e}(t)=\frac{v\left(t^{+}\right)+e v\left(t^{-}\right)}{1+e}, x=\left(q^{T}, v^{T}\right)^{T}$ is the system's state, $d v$ is the differential measure of the right-continuous function $v(\cdot), e \in[0,1]$ is a restitution coefficient. The dynamics in (2.6) is an MDI, i.e., the left-hand side in (2.6c) is a measure included in the normal cone. The admissible domain is the finitely represented set $\Phi=\{q \in$ $\left.\mathbb{R}^{\frac{n}{2}} \mid h(q) \geq 0\right\}$, where $h_{i}: \mathbb{R}^{\frac{n}{2}} \rightarrow \mathbb{R}, 1 \leq i \leq m$, are continuously differentiable functions such that $\nabla h(q) \neq 0$ on $\operatorname{bd}(\Phi)$. The tangent cone to $\Phi$ at $q$ is denoted by $\mathcal{T}_{\Phi}(q)$ and the closed convex polyhedral cone $V(q)$ appearing in (2.6), is the tangent linearization cone $\mathcal{T}_{\Phi}^{h}(q)^{1}$. That is (see (A.3)),

$$
V(q)=\left\{v \in \mathbb{R}^{\frac{n}{2}} \mid v^{T} \nabla h_{i}(q) \geq 0, i \in \mathcal{I}(q)\right\} \quad\left(=\mathcal{T}_{\Phi}^{h}(q)\right),
$$

\footnotetext{
${ }^{1}$ Here we keep the notation $V(q)$ adopted by J.J. Moreau in his articles.
} 
and $\mathcal{I}(q) \triangleq\left\{i \in 1, \ldots, m \mid h_{i}(q) \leq 0\right\}$ is the set of indices of active constraints. Thus, a SOSwP can be seen as a particular case of (1.1) with

$$
\mathcal{F}(t, H x, J w)=\mathcal{N}_{V(q)}\left(v_{e}\right), \quad f(t, x)=\left(\begin{array}{c}
v \\
F(t, q, v)
\end{array}\right) \quad G(t, x)=\left(\begin{array}{c}
0 \\
I_{n}
\end{array}\right) .
$$

In this case $w \in \mathbb{R}^{n}$ represents the generalized contact forces. If the unilateral constraints are properly defined with the signed gap functions, constructed from the socalled local kinematics $[4,130]$, then $w=\nabla h(q) \lambda_{\mathrm{n}}, \lambda_{\mathrm{n}} \in \mathbb{R}^{m}$ is the vector of Lagrange multipliers associated with the unilateral constraints, and represents the contact forces in the local frames. When $q(t) \in \operatorname{int}(\Phi)$ then $V(q(t))=\mathbb{R}^{n} \Rightarrow \mathcal{N}_{V(q)}(\cdot)=\{0\}$, when $q(t) \in \operatorname{bd}(\Phi)$ and $v_{e}(t) \in \operatorname{int}\left(V(q(t))\right.$ then $\mathcal{N}_{V(q)}\left(v_{e}(t)\right)=\{0\}$. Outside discontinuity times, $v_{e}(t)=v\left(t^{+}\right)=v(t)$ (solutions are right-continuous). The major difference between (2.2) and (2.6) is that the convex set is the tangent cone $V(q)$, which depends on the position $q$, while the normal cone is calculated at the velocity variable $v_{e}$. Solutions of (2.6) therefore possess an intrinsic non-regularity $(v(\cdot)$ jumps at impact times when $\nabla h(q(t))^{T} \dot{q}\left(t^{-}\right)<0$ and $h(q(t))=0$, see Figure $2.1 \mathrm{~b}$ ), while solutions of $(2.2)$ are continuous if $\mathcal{S}(\cdot)$ is (even in case of state-dependent $\mathcal{S}(t, x)[37,293,370]$ ). As we shall see later, the sweeping process order is intimately related to the relative degree between two complementary variables which rule the contact/non contact with $\operatorname{bd}(\Phi)$ phases of motion. To get a quick glance into these complementarity variables for system (2.6), we introduce the outward linearization normal cone to $\Phi$ at $q$, given by $\mathcal{N}_{\Phi}^{h}(q) \triangleq(V(q))^{\circ}$, which by definition of $V(q)$, is seen to be generated by $\nabla h_{i}(q), i \in \mathcal{I}(q)$ (see Appendix A). If an element $\eta \in \mathcal{N}_{\Phi}^{h}(q)$ is represented as $\eta \triangleq \sum_{i=1}^{m} \lambda_{\mathrm{n}, i} \nabla h_{i}(q)$, then the underlying complementary problem is made of the complementarity conditions

$$
0 \leq \lambda_{\mathrm{n}, i} \perp h_{i}(q) \geq 0, \quad 1 \leq i \leq m,
$$

so that the function $h_{i}(q)$ and the multiplier $\lambda_{\mathrm{n}, i}$ represent the two complementary variables. It also holds that $\mathcal{N}_{V(q)}\left(v_{e}\right) \subseteq \mathcal{N}_{\Phi}^{h}(q)$ (see [130, Appendix B.2.2]), and so we see from (2.6c) that the second time derivative of $h_{i}(q)$ contains $\lambda_{\mathrm{n}, i}$. This can be interpreted as a relative degree 2 for the underlying system with $\lambda_{\mathrm{n}, i}$ as input, and $h_{i}(q)$ as output. Some details are provided in Sections 4.2.1 and Appendix C for relative degree issues. The rationale behind Moreau's set in the right-hand side of (2.6c) is further clarified in Section 4.2.1, see Figure 4.3 (b) where one sees that it imposes a lexicographical inequality for the position and velocity constraints (see Appendix E for a definition). See [130, Chapter 5], [4] and [427, 372, 443] for more details on the SOSwP, its state jump mapping, its well-posedness and its time-discretization (which we will review later in this article).

SOSwP with AC solutions. A different class of SOSwP is introduced in $[168,169]$ (see [427, Chapter 5] for an exposition), as: $\ddot{u} \in-\mathcal{N}_{\mathcal{S}(u)}(\dot{u})$, with $\mathcal{S}(u)$ Lipschitz and bounded, and Lipschitz continuous solution $(u(\cdot), \dot{u}(\cdot))$. An extension is analyzed in [339]. It takes the form in the finite dimensional case: $\ddot{u} \in-B \mathcal{N}_{\mathcal{S}(t, u, \dot{u})}(\dot{u})+$ $F(t, u, \dot{u})+A u$, with $F(\cdot)$ set-valued with closed convex values, while the closed set $\mathcal{S}(\cdot)$ is uniformly subsmooth and satisfies Lipschitz-like and compactness properties. Solutions are such that $u(\cdot), \dot{u}(\cdot)$ and $\ddot{u}(\cdot)$ are Lebesgue integrable and $\dot{u}(\cdot)$ is AC: we are therefore in a totally different framework than (2.6). Results for this sort of SOSwP can be found in [43] who extend the well-posedness analysis made in [171, 170] to similar SOSwP with prox-regular, compact sets $\mathcal{S}(u)$, where $F(t, u, \dot{u})$ is set-valued, 
not necessarily bounded. See also $[47,66]$ for similar studies, where $\mathcal{S}(u)$ is Lipschitz, closed, prox-regular. The DI: $\ddot{u} \in-\mathcal{N}_{\mathcal{S}(t, u)}(\dot{u})$ is analysed in [115], with Lipschitz solutions, $\mathcal{S}(\cdot, \cdot)$ prox-regular Lispchitz sets. Upper semicontinuous convex-valued perturbations $F(t, u, \dot{u})$ and convex or prox-regular sets $\mathcal{S}(t, u)$ are considered in [37, 40], convex compact Lipschitz multifunctions $\mathcal{S}(u)$, and a set-valued perturbation with memory $F(t, T(t) u)$ are analysed in [292], equi-uniformly subsmooth sets $\mathcal{S}(t, u)$ (compact in the finite-dimensional case) are considered in [46], prox-regular AC and Lipschitzean $\mathcal{S}(t, u)$ and Lipschitz perturbations with linear growth are studied in [399]. Again, solutions of all these SOSwP are AC, or Lipschitz continuous, or BV continuous. We will give an insight of the deep discrepancy between this kind of SOSwP and (2.6), later in the article (see Section 3.7). Time-delays for such SOSwP have been considered in $[450,117]$.

2.1.3. Higher order sweeping process (HOSwP). As a natural extension of the FOSwP and SOSwP, the HOSP was introduced in [6]. Just like in SOSwP, with $q$ constrained to evolve in $\Phi$, the measure $\eta$ entered in the model equations in the second derivative of $q$, we can think of an $\mathrm{HOSwP}$, where some component of the state is constrained but the complementarity variable only enters in the dynamics via some higher-order derivative of the constrained variable. One way to formalize this idea, is to consider the system $\dot{x}=A x+B \lambda, C x \geq 0, A \in \mathbb{R}^{n \times n}, B \in \mathbb{R}^{n \times m}, C \in \mathbb{R}^{m \times n}$ and to design a suitable set-valued mapping $\mathcal{F}(x)$ so that trajectories remain in the admissible domain $\left\{x \in \mathbb{R}^{n} \mid C x \geq 0\right\}$. To this aim, it is convenient to work in a canonical state space with new state $z=W x, W \in \mathbb{R}^{n \times n}$ full rank, $z_{1}=C x$, that transforms the system to (see Appendix C):

$$
\left\{\begin{aligned}
\dot{z}_{1} & =z_{2}, \dot{z}_{2}=z_{3}, \ldots, \dot{z}_{r-1}=z_{r} \\
\dot{z}_{r} & =C A^{r} W^{-1} z+C A^{r-1} B \lambda \\
\dot{\zeta} & =A_{0} \zeta+B_{0} z_{1} \\
z_{1} & \geq 0
\end{aligned}\right.
$$

where $\bar{r}=(r, \ldots, r)^{T} \in \mathbb{R}^{m}, 1 \leq r$ and $r m \leq n$, is the vector relative degree of the triple $(A, B, C), z=\left(z_{1}^{T}, \ldots, z_{r}^{T}, \zeta^{T}\right)^{T}$, the $\zeta$-dynamics in $(2.8 \mathrm{c})$ is the so-called zero-dynamics which represents the system's behaviour on the submanifold $z_{1}=0$. A detailed presentation of the functional framework for $\mathrm{HOSwP}$ would be much too long. Let us mention that solutions are Schwartz' distributions constructed from the differentiation of RCLSBV functions (hence the HOSwP is basically a Distribution Differential Inclusion (DDI)), whose degree as distributions depends directly on $r[6$, Proposition 3]. This is easily seen as follows when $m=1$ : assume that $z_{1}(0)=0$, $z_{3}(0)=z_{4}(0)=\cdots=z_{r}(0)=0$ while $z_{2}\left(0^{-}\right)<0$. The only way to guarantee that $z_{1}(t) \geq 0$ in a right-neighborhood of $t=0$, is to impose $z_{2}\left(0^{+}\right) \geq 0$. This jump propagates in the chain (2.8a) until (2.8b), showing that $z_{3}$ is a Dirac measure, $z_{4}$ is a Dirac derivative, and so on, so that $\lambda$ is a distribution of degree $r+1$. However an equivalent MDI formulation exists [6, Section 4.2] which we use next, for which solutions are measures. The important point for us is that the chain of integrators in $(2.8 \mathrm{a})$ and $(2.8 \mathrm{~b})$ is constrained to satisfy an MDI of the form:

$$
\left\{\begin{array}{l}
z_{1}(t) \in \Phi_{m} \text { for all } t>0 \\
d z_{i}-z_{i+1}(t) d t \in-\mathcal{N}_{\mathcal{T}_{m}^{i-1}\left(Z_{i-1}\left(t^{-}\right)\right)}\left(z_{e, i}(t)\right), 1 \leq i \leq r-1 \\
d z_{r}-C A^{r} W^{-1} z d t \in-\left(C A^{r-1} B\right) \mathcal{N}_{\mathcal{T}_{\Phi_{m}}^{r-1}\left(Z_{r-1}\left(t^{-}\right)\right)}\left(z_{e, r}(t)\right)
\end{array}\right.
$$


where $Z_{i}=\left(z_{1}, z_{2}, \ldots, z_{i}\right)$, the tangent cones are defined as $\mathcal{T}_{\Phi_{m}}^{i}\left(Z_{i}\right)=\mathcal{T}_{\mathcal{T}_{\Phi_{m}}^{i-1}\left(Z_{i-1}\right)}\left(z_{i}\right)$, $1 \leq i \leq r, Z_{0}=0$ and $\mathcal{T}_{\Phi_{m}}^{0}\left(Z_{0}\right)=\Phi_{m}, \Phi_{m}=\mathbb{R}_{+}^{n}, z_{e, i}(t)=\frac{z_{i}\left(t^{+}\right)+e_{i} z_{i}\left(t^{-}\right)}{1+e_{i}}$ mimics the variable $v_{e}$ in $(2.6 \mathrm{c})$, and the $e_{i}$ 's may be thought of as restitution coefficients in an extended framework.

EXAMPLE 1. Consider system (2.9) for the case $m=1$. In this case, we observe that, by definition, $\mathcal{T}_{\Phi_{m}}^{0}\left(Z_{0}\right)=\Phi_{m}=\mathbb{R}_{+}$, and

$$
\begin{gathered}
\mathcal{T}_{\Phi_{m}}^{1}\left(Z_{1}\right)=\mathcal{T}_{\Phi_{m}}^{1}\left(z_{1}\right)= \begin{cases}\mathbb{R} & \text { if } z_{1}>0, \\
\mathbb{R}_{+} & \text {if } z_{1}=0\end{cases} \\
\mathcal{T}_{\Phi_{m}}^{2}\left(Z_{2}\right)=\mathcal{T}_{\Phi_{m}}^{2}\left(z_{1}, z_{2}\right)= \begin{cases}\mathbb{R} & \text { if } z_{1}>0 \\
\mathbb{R} & \text { if } z_{1}=0, z_{2}>0 \\
\mathbb{R}_{+} & \text {if } z_{1}=0, z_{2}=0 .\end{cases}
\end{gathered}
$$

In $(2.9 \mathrm{~b})(2.9 \mathrm{c}), d z_{i}$ is the differential measure associated with the function $z_{i}$. From (2.8) and (2.9) we have:

$$
\mathcal{F}(t, H z, J w)=\left(\begin{array}{c}
\mathcal{N}_{\mathcal{T}_{\Phi_{m}}^{0}\left(Z_{0}\left(t^{-}\right)\right.}\left(z_{e, 1}\right) \\
\mathcal{N}_{\mathcal{T}_{m}^{1}\left(Z_{1}\left(t^{-}\right)\right.}\left(z_{e, 2}\right) \\
\vdots \\
\mathcal{N}_{\mathcal{T}_{\Phi_{m}}^{r-1}\left(Z_{r-1}\left(t^{-}\right)\right.}\left(z_{e, r}\right)
\end{array}\right), \quad f(t, z)=\left(\begin{array}{c}
z_{2} \\
\vdots \\
z_{r} \\
C A^{r} W^{-1} z \\
A_{0} \zeta+B_{0} z_{1}
\end{array}\right)
$$

with $w=\left(w_{1}, \ldots, w_{r}\right)^{T}$, and $G(t, z)=\left(\begin{array}{cc}I_{(r-1) m} & 0 \\ 0 & C A^{r-1} B \\ 0 & 0\end{array}\right) \in \mathbb{R}^{n \times r m}$ (the zero dynamics is an ODE). In fact, it happens that outside the state reinitialization times, one has $w_{1}=w_{2}=\ldots=w_{r-1}=0$ (that is, the first $r-1$ multipliers or selections inside the normal cones play a role only as distributions to keep the "output" inside the admissible domain $\Phi_{m}$ ). In other words, the measures $d \nu_{i}=d z_{i}-z_{i+1} d t=$ $\chi_{i}(t) d t+d \mathcal{J}_{i}$ satisfy $\chi_{i}(t) \equiv 0$ for all $1 \leq i \leq r-1$, where $d \mathcal{J}_{i}$ is an atomic measure with countable set of atoms generated by a right-continuous jump function $\mathcal{J}_{i}(\cdot)$. In the framework of (1.1) which is stated only outside state jumps, it is better to set

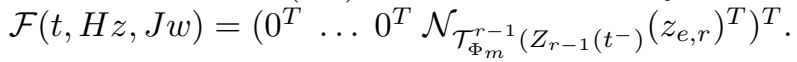

One major assumption for the well-posedness is that $C A^{r-1} B \in \mathbb{R}^{m \times m}$ is a Stieltjes matrix. The inclusions in (2.9b) (2.9c) imply the componentwise lexicographical inequality $\left(z_{1}\left(t^{+}\right), \ldots, z_{r}\left(t^{+}\right)\right) \succcurlyeq 0[6$, Remark 16]. Roughly speaking, in the same way as Moreau's set in the SOSwP (2.6c) takes care of rectifying the velocity (when needed) in order to keep trajectories inside the position admissible domain $\Phi$, the set-valued right-hand side in the HOSwP takes care (when needed) of rectifying some derivatives of $z_{1}$ to keep $z_{1}$ inside $\Phi_{m}$. The above HOSwP framework has been extended to the non-autonomous case in [132]. Globally, the well-posedness proof remains very close to that of the autonomous case.

2.1.4. Comments. The SOSwP is taylored to nonlinear Lagrangian systems, while the HOSwP essentially applies to linear invariant systems with linear constraints (the product of distributions of degree $\geq 2$ being mathematically unclear). However it is apparent from $(2.4),(2.6 \mathrm{~b})(2.6 \mathrm{c})$, and $(2.9 \mathrm{~b})(2.9 \mathrm{c})$, that there is a logic which prevails in the definition of the first order, second order and higher order sweeping processes: 
- their right-hand sides comprise normal cones to tangent cones of increasing "order", and this "order" is directly related to the relative degree that can be defined in an equivalent complementarity formalism (when it exists). Let us adopt the above convention that for a set $\Phi$ we have $\mathcal{T}_{\Phi}^{0}=\Phi$. In (2.2) the set-valued term is $\mathcal{N}_{\mathcal{T}_{\Phi}^{0}(x)}(x)$ with $\Phi=\mathcal{S}(t)$, in $(2.6)$ this is $\mathcal{N}_{\mathcal{T}_{\Phi}(q)}(v)$, and in $(2.9) \mathcal{F}(t, H z, J w)$ contains a sequence of normal cones $\mathcal{N}_{\mathcal{T}_{\Phi}^{i}\left(Z_{i}\right)}$ with $\Phi=\Phi_{m}$.

- As a consequence there is a common feature between $(2.2),(2.6)$ and (2.9): their set-valued right-hand sides define, for fixed $t$ in (2.2) (b), fixed $q$ in (2.6) (b), and fixed $Z_{i-1}\left(t^{-}\right)$in (2.9), maximal monotone mappings. Let $\mathcal{S}(t)$ be closed convex in (2.2), let also $C A^{r-1} B \succ 0$ in (2.9c). Hence (A.6) applies. This has important consequences at times of discontinuity in the state. Consider first the MDI (2.4). Assuming that its solutions are right-continuous, at a state discontinuity time $t$, the dynamics is equivalent to

$$
x\left(t^{+}\right)-x\left(t^{-}\right) \in-\mathcal{N}_{\mathcal{S}(t)}\left(x\left(t^{+}\right)\right) .
$$

This is a generalized equation with unknown $x\left(t^{+}\right)$whose solution is unique when $\mathcal{S}(t)$ is closed convex as: $x\left(t^{+}\right)=\operatorname{proj}\left[\mathcal{S}(t) ; x\left(t^{-}\right)\right]$, see (A.6) in Section A.2.2. We note that depending on $\mathcal{S}(t)$ this projection may not be easy to compute, but there are important cases for which it can be (see for instance Section 2.4.4). Consider now (2.9b) (2.9c), at a discontinuity of $z_{i}$ (which is an atom of $d z_{i}$ ) one gets:

$$
\begin{array}{ll} 
& z_{i}\left(t^{+}\right)-z_{i}\left(t^{-}\right) \in-\mathcal{N}_{\mathcal{T}_{\Phi}^{i-1}\left(Z_{i-1}\left(t^{-}\right)\right.}\left(z_{e, i}(t)\right), 1 \leq i \leq r-1, \\
\Longleftrightarrow \quad & z_{i}\left(t^{+}\right)=-e_{i} z_{i}\left(t^{-}\right)+\left(1+e_{i}\right) \operatorname{proj}\left[\mathcal{T}_{\Phi}^{i-1}\left(Z_{i-1}\left(t^{-}\right)\right) ; z_{i}\left(t^{-}\right)\right] \\
\text {and } \quad & z_{r}\left(t^{+}\right)-z_{r}\left(t^{-}\right) \in-\left(C A^{r-1} B\right) \mathcal{N}_{\mathcal{T}_{\Phi}^{r-1}\left(Z_{r-1}\left(t^{-}\right)\right)}\left(z_{e, r}(t)\right) \\
\Longleftrightarrow \quad & z_{r}\left(t^{+}\right)=-e_{r} z_{r}\left(t^{-}\right)+\left(1+e_{r}\right) \operatorname{proj}_{\left(C A^{r-1} B\right)^{-1}}\left[\mathcal{T}_{\Phi}^{r-1}\left(Z_{r-1}\left(t^{-}\right)\right) ; z_{r}\left(t^{-}\right)\right] .
\end{array}
$$

In (2.6c), at a velocity jump (which is an atom of $d v$ ) we obtain $v\left(t^{+}\right)-v\left(t^{-}\right) \in$ $-\mathcal{N}_{V(q(t))}\left(v_{e}\right)$, hence $v\left(t^{+}\right)=-e v\left(t^{-}\right)+(1+e) \operatorname{proj}\left[V(q(t)) ; v\left(t^{-}\right)\right]$. One can say that sweeping processes intrinsically define state jump mappings with the same structure (independently of the fact, whether or not these mappings possess a physical meaning).

- Jump maps in FOSwP with BV solutions can be extended from (2.12), by taking into account "intermediate" sets between $\mathcal{S}\left(t^{-}\right)$and $\mathcal{S}\left(t^{+}\right)$[488], in a way similar to filling-in the jumps by vertical segments in discontinuous functions. This is in fact already present in a particular case in the $\mathrm{HOSwP}_{\text {at }}$ the level of the variable $z_{1}$ in (2.13).

- Let us apply the SOSwP to a linear Lagrangian system with $h(q)=C q$, and rewrite (2.6b) (2.6c) in an HOSwP framework with $z_{1}=C q$ and $z_{2}=C v$. It reads as $d z_{1}=z_{2}, d z_{2}-f\left(t, z_{1}, z_{2}\right) \in-\mathcal{N}_{\mathcal{T}_{\Phi_{m}}^{1}}\left(Z_{1}\left(t^{-}\right)\right)\left(z_{e, 2}\right)$ : the absence of the set-valued right-hand side $\mathcal{N}_{\mathcal{T}_{\Phi}^{0}\left(Z_{0}\left(t^{-}\right)\right)}\left(z_{e, 1}\right)$ is a consequence of Moreau's viability Lemma [130, Lemma 5.1] [442], which states that if $q(0) \in \Phi$ and $v(t) \in V(q(t))$ Lebesgue almost everywhere, then $q(t) \in \Phi$ for all $t \geq 0$. Hence a jump in $z_{1}$ (i.e., in the position $q$ ) could occur only initially if $q\left(0^{-}\right) \notin \Phi$ : in Contact Mechanics such a case is never considered for obvious physical reasons. Consequenlty there is no need to resort to a non zero multiplier inside $\mathcal{N}_{\Phi}\left(z_{1}\right)$ (see also [6, Remark 14]).

- In this section we have taken the point of view of the structure of the set-valued right-hand sides to classify the sweeping processes of increasing order. As alluded 
to above, the notion of relative degree between two suitable complementarity variables is also an underlying fundamental characterisitic, see Sections 3.4 and 4.2.1 for more details on this aspect for the FOSwP and SOSwP.

- The SOSwP in (2.6c) and the HOSwP (2.9b) (2.9c) could be written equivalently as we did for the FOSwP in (2.3) with suitable measure $d \mu$ [427] [6, p.160].

- The notion of relative degree will be often met in this article. However in our setting we will require that the so-called decoupling matrix (equal to $C A^{r-1} B$, familiar to Control scientists in the input/output linearization problem [553, p. 460]) not only be full-rank, but also possesses positivity-like properties (P-matrices and variations [210], $\succ 0$ and $\succcurlyeq 0$ matrices, co-positive matrices, Stieltjes matrices etc).

- Solutions usually do not depend continuously on the initial data in SOSwP and HOSwP, except when the constraints satisfy some angle restrictions [464, 73, 130]. Solutions of the FOSwP, with prox-regular AC sets $\mathcal{S}(t)$, Lipschitz continuous $f(t, x)$ satisfying a linear growth condition, are such that the map $x_{0} \mapsto x_{x_{0}}(\cdot)$, which associates with each $x_{0} \in \mathcal{S}(0)$ the unique solution of the FOSwP with $x(0)=x_{0}$, is Lipschitz continuous [237, Proposition 2].

- The set $\mathcal{S}(t)$ may take various forms: $\mathcal{S}(t)=\mathcal{S}+u(t), \mathcal{S}=\left\{x \in \mathbb{R}^{n} \mid C x \geq 0\right\}$ [162], $\mathcal{S}(t)=\left\{x \in \mathbb{R}^{n} \mid C(t) x+B(t) \geq 0\right\}, C(\cdot)$ and $B(\cdot)$ AC [201], $\mathcal{S}(t)=\cap_{i} \mathcal{S}_{i}+u(t)$, $\mathcal{S}_{i}=\left\{x \in \mathbb{R}^{n} \mid k_{i}(x) \geq 0\right\}, 1 \leq i \leq m[161], \mathcal{S}(t)=\left\{x \in \mathbb{R}^{n} \mid \Psi(t, x) \in \mathcal{S}\right\}, \mathcal{S}$ locally bounded, $\Psi(t, \cdot) \in C^{2}[316], \mathcal{S}(t)=\left\{\left(x_{1}, x_{2}\right) \in \mathbb{R}^{2} \mid\left(x_{1}-w_{1}(t)\right)^{2}+\left(x_{2}-\right.\right.$ $\left.\left.w_{2}(t)\right)^{2} \geq 1\right\}, w_{i}(t)$ sample paths of Brownian motion with unbounded variation $[204], \mathcal{S}(t)=\left\{(x, y) \in \mathbb{R}^{2} \mid x^{2}+y^{2} \geq r(t)\right\}, r(t)=\max \left(f(t), \frac{1}{2}\right), f:[0,1] \rightarrow[0,1]$ the Cantor function [204].

- There is a close link between FOSwP and so-called Skorokhod problems. Indeed in the deterministic case, the Skorokhod problem boils down to a FOSwP: $-\dot{x} \in$ $\dot{h}(t)+\mathcal{N}_{\mathcal{S}(t)}(x)$, with $\mathcal{S}(t)=h(t)+K$, where $K$ is a fixed polyhedral set, and $h(\cdot)$ is differentiable $[365,364]$.

- A last comment is, as alluded to above, that there is a drastic difference between state dependent sets $\mathcal{S}(t, x)$ as in $[293,37,370]$, and the sets in the SOSwP and the HOSwP. Indeed in $[293,37,370] \mathcal{S}(t, x)$ is assumed to satisfy Lipschitz-like continuity in both arguments, which does not hold for tangent cones.

2.1.5. Perspectives. The research about FOSwP and SOSwP is a very active area, as witnessed by the number of recent articles cited in Section 2.1.1. Variants will continue to be studied by changing the properties of the aforementioned basic ingredients. Stochastic FOSwP could still offer open problems. It is however possible that the major unexplored fields for the FOSwP, are stability and invariance of equilibria, sets, and trajectories $[300,556]$, dynamical analysis (existence of periodic solutions $[172,173,343,344]$, bifurcations and chaos), and Systems and Control aspects (optimal control and the maximum principle [54, 160, 162, 161, 200, 202, 201, 205, 316, 217,536 ], controllability [122], observability, state observer design [522], trajectory tracking, etc). An important issue concerns the extension of the design of observers for time-dependent FOSwP $[138,522]$, to state-dependent FOSwP with right-hand side $\mathcal{N}_{\mathcal{S}(x, t)}(x)$. Indeed the estimated set $\mathcal{S}(\hat{x}, t)$ and the plant's set $\mathcal{S}(x, t)$ differ, in particular at eventual jump times, as well as the two normal cones. This creates additional difficulty in the stability analysis. One path could be to use the distance defined in (5.15), and a Lipschitz constraint on $d_{\text {haus }}(\mathcal{S}(\hat{x}, t), \mathcal{S}(x, t))$. Clearly the way the control input enters the dynamics (in the perturbation $f(t, x, u)$, or in the set $\mathcal{S}(t, u)$, or both) strongly influences the subsequent analysis. So-called Lyapunov 
pairs $[556,301,300]$ (which are made of a pair of functions satisfying a dissipation-like inequality similar to (D.2)), could be useful in this setting, though they have been used mainly for well-posedness analysis. In summary, the dynamical and control-oriented analysis of FOSwP: $\dot{x}-f(t, x, u) \in-\mathcal{N}_{\mathcal{S}(t, u)}(x)$, where $u(\cdot)$ is some feedback control, is a source of many open problems. Time delays in FOSwP and SOSwP may, or may not, be related to control issues, and seem to remain a largely open issue. Again, the delays may appear at various places: in the perturbation $f\left(t, x\left(t-\tau_{1}\right)\right)$, in the moving set $\mathcal{S}\left(t-\tau_{2}, x\left(t-\tau_{3}\right)\right)$, in the normal cone argument $\mathcal{N}_{\mathcal{S}(t)}\left(x\left(t-\tau_{4}\right)\right)$, in the derivative $\dot{x}\left(t-\tau_{5}\right)$, giving rise to several classes of retarded differential inclusions. The analysis in $[115,294,450,174,236,235]$ focus on delays in the perturbation. Finally, singular FOSwP: $P \dot{x}-f(t, x) \in-\mathcal{N}_{\mathcal{S}(t, x)}(x)$, with $P$ a singular matrix, and their relationship with switching DAE and MLCS as in (2.25) below, is a topic of interest in some hydraulic/thermodynamical models. Concerning the HOSwP, interesting extensions could concern the consideration of vector relative degrees $\bar{r}=\left(r_{1}, \ldots, r_{m}\right)^{T}, r_{i} \neq r_{j}$ when $i \neq j$, study cases of globally well-posed nonlinear HOSwP (involving products of distributions) relying on nonstandard analysis [101], analyse the case of mixed unilateral and bilateral (equality) constraints, and analyze switching between HOSwPs with varying state dimension (switching dynamic feedback controllers).

2.2. Maximal monotone differential inclusions. In the formalism of sweeping processes, we saw that the set-valued right-hand side in the DIs is determined by the normal cone operators. However, a more general class of such dynamical systems allows us to make connections with differential inclusions where the set-valued mapping on the right-hand side is maximal monotone with respect to the state variable. To see such a connection, consider the dynamical system

$$
\begin{gathered}
\dot{x}=f(t, x(t))+\eta(t) \\
v(t)=C x(t)+D \eta(t), \quad v(t) \in \mathcal{S}(t), \\
\left\langle v^{\prime}-v(t), \eta(t)\right\rangle \geq 0, \quad \forall v^{\prime} \in \mathcal{S}(t),
\end{gathered}
$$

where $\mathcal{S}:[0, \infty) \rightrightarrows \mathbb{R}^{n}$, with $\mathcal{S}(t)$ closed, convex, and nonempty, for each $t \geq 0$. The constraint in $(2.14 \mathrm{c})$ is a variational inequality (VI). The vector field $f:[0, \infty) \times \mathbb{R}^{n} \rightarrow$ $\mathbb{R}^{n}$ is Lebesgue integrable in time (the first argument), and globally Lipschitz in the state variable (with possibly time-varying Lipschitz modulus) and $D \in \mathbb{R}^{n \times n}$ is a positive semidefinite matrix.

Recalling from section A.2.4 the relation that the support function $\sigma_{\mathcal{S}}(\cdot)$ is the conjugate of the indicator function $\psi_{\mathcal{S}}(\cdot)$, it is observed that

$$
\begin{aligned}
& \left\langle v^{\prime}-v(t), \eta(t)\right\rangle \geq 0, \quad \forall v^{\prime} \in \mathcal{S}(t) \\
& \Leftrightarrow \eta(t) \in-\partial \psi_{S(t)}(C x(t)+D \eta(t)) \Leftrightarrow C x(t)+D \eta(t) \in \partial \sigma_{\mathcal{S}(t)}(-\eta(t)) \\
& \Leftrightarrow C x(t) \in\left(\partial \sigma_{\mathcal{S}(t)}+D\right)(-\eta(t)) \Leftrightarrow-\eta(t) \in\left(\partial \sigma_{\mathcal{S}(t)}+D\right)^{-1}(C x(t)) .
\end{aligned}
$$

Thus, if we introduce the operator $\mathcal{F}:[0, \infty) \times \mathbb{R}^{n} \rightrightarrows \mathbb{R}^{n}$, to be $\mathcal{F}(t, \cdot)=\left(\partial \sigma_{\mathcal{S}(t)}+\right.$ $D)^{-1}(C \cdot)$, then system $(2.14)$ can be equivalently written as

$$
\dot{x} \in f(t, x)-\mathcal{F}(t, x),
$$

with the initial condition $x(0) \in \operatorname{dom}(\mathcal{F}(0, \cdot))$. Let $C=I_{n}$. Then it is an easy exercise to show that the operator $\mathcal{F}(t, \cdot)$ is maximal monotone for each $t \geq 0$. Hence, we have a class of DIs described by the sum of a Lipschitz vector field and a time-dependent set-valued mapping, where the latter is maximal monotone with respect to the state 
of the system, for each $t \geq 0$. By choosing $\mathcal{F}(t, x)=\mathcal{N}_{\mathcal{S}(t)}(x)$, we see that (2.16) is a generalization of the FOSwP with closed convex $\mathcal{S}(t)$.

The solution theory for DIs (2.16) with time-invariant maximal monotone operators $\mathcal{F}: \mathbb{R}^{n} \rightrightarrows \mathbb{R}^{n}, x \mapsto \mathcal{F}(x)$, has been extensively studied since the work of Brézis [123], see e.g., [100]. Dealing with general time-varying maximal monotone DIs is more complex. Some of the earlier works in this direction [123, 346, 470] only allow us to deal with the inclusion $\dot{x} \in-\mathcal{F}(x)+\gamma x-b(t)$, where $\mathcal{F}(\cdot)$ is maximal monotone, $\gamma>0$ is a scalar, and $b:[0, \infty) \rightarrow \mathbb{R}^{n}$ is AC. Extensions to systems with $\gamma x$ replaced by the vector field $f(t, x)$ satisfying Lipschitz continuity in $x$ and boundedness conditions on the Jacobian may be found in $[84,86,92]$. Both articles $[179,121]$ relax the maximal monotonicity and consider that $\mathcal{F}(x)=\partial g(x)-F(x)$ with $g(\cdot)$ proper convex LSC and $F(x)$ cyclically monotone $(\Leftrightarrow F(x) \subseteq \partial V(x)$ for some proper convex LSC $V(\cdot))$. The case when the right-hand side is of the form $\Gamma(x) \mathcal{F}(x)$ for some matrix function $\Gamma(x)=$ $\Gamma^{T}(x) \succcurlyeq 0$ for all $x$, is analysed in $[17,422,421]$, for robust control applications. Here the operator $x \mapsto \Gamma(x) \mathcal{F}(x)$ is not necessarily monotone. The case when $\mathcal{F}(t, x)=$ $\partial \varphi_{1}(x)-\varphi_{2}(x), \varphi_{i}(\cdot)$ proper convex LSC functions, is analyzed in [537, 456], with $f(t, x)=A(t, x) u(t), u(t) \in U(t, x)$ for some set-valued mapping $U(\cdot)$ in [537]. It is also worth mentioning the results in $[126,135]$ and $[159]$, which deal with the negative feedback interconnection of a passive dynamical linear system $(A, B, C, D)$ (see Definition D.1) and a maximal monotone operator $\mathcal{M}(\cdot)$ (more specifically, [126, 135] consider $\mathcal{M}(\cdot)=\partial \varphi(\cdot)$ for a convex proper $\operatorname{LSC} \varphi(\cdot))$ :

$$
\left\{\begin{array}{l}
\dot{x}=A x+B \lambda \\
z=C x+D \lambda \\
\lambda \in-\mathcal{M}(z),
\end{array} \Leftrightarrow \dot{x} \in\left(A-B\left(D+\mathcal{M}^{-1}\right)^{-1} C\right)(x) .\right.
$$

The link with (2.14) and (2.16) is easily made. Here $w=\lambda$ and $\mathcal{F}(\cdot)=\mathcal{M}(\cdot)$. It is shown in $[126,135,159]$ that such an interconnection defines a new operator that is also maximal monotone. Therefore, this can be considered as a new operation that preserves maximal monotonicity, in addition to the classical ones [97, Proposition 20.22, 20.23, Theorem 24.2]. This is treated in an infinite-dimensional framework in $[29,28]$, and in a nonlinear case $(A x$ is replaced by $A(x), A(\cdot)$ Lipschitz) in [136].

Proposition 2.1. Assume that the system $(\Sigma)$ represented by the quadruple $(A, B, C, D)$ is passive, and that the set-valued mapping $\mathcal{M}: \mathbb{R}^{n} \rightrightarrows \mathbb{R}^{n}$ is maximal monotone. Assume further that $\operatorname{Im}(C) \cap \operatorname{rint}\left(\operatorname{Im}\left(D+\mathcal{M}^{-1}\right)\right) \neq \emptyset$. Then the operator $x \mapsto-\left(A-B\left(D+\mathcal{M}^{-1}\right)^{-1} C\right)(x)$, obtained by the negative feedback interconnection of the system $(A, B, C, D)$ and $\mathcal{M}(\cdot)$, is maximal monotone.

Thus the feedback passification of the controlled system with $\dot{x}=A x+B \lambda, z=$ $C x+D \lambda$ in (2.17), allows one to recast the closed-loop system into maximal monotone DIs.

The domain of the set-valued operator does not change with time in the above results. The first real contribution in the literature with time-varying domains is observed in the seminal work of J.J. Moreau [434, 436, 435, 439], as discussed in detail in previous sections. The systems studied here within the umbrella of sweeping process, comprise DIs with a special conic structure. Sweeping processes provide the first instance in the literature of inclusion with a particular kind of maximal monotone operators which depend on time, and the corresponding domain may vary. The analysis in [348] deals with $\mathcal{F}(t, x)=\partial \varphi(t, x), \varphi(t, \cdot)$ proper LSC for each $t$, and $f(t, x)=f(t)$. Existence and uniqueness of AC solutions hold under some assumptions on its Yosida approximation. The case $\mathcal{F}(t, H x)$ for some strongly monotone operator $H=\partial \phi, \phi(\cdot)$ 
continuous convex, is also treated in [348] (this is similar to the degenerate FOSwP in Section 2.1.1). To generalize the work of sweeping process, [560] studied more general evolution inclusions with time-varying domains, with the constraint that the set-valued mapping $\mathcal{F}(t, \cdot)$ is just maximal monotone for each $t \geq 0$, under certain regularity assumption. The assumptions were later relaxed by [368], and further relaxed in [124], replacing global estimates by local ones. Other results concerning the case $\mathcal{F}(t, x)=\partial \varphi(t, \cdot)$ with $\varphi(t, \cdot)$ proper convex LSC for each $t$, and with a set-valued, convex or non-convex, possibly unbounded $f(t, x)$ in $(2.16)$, are obtained in $[538,540]$ as a continuation of studies on polyhedral FOSwP with set-valued perturbations. All the above systems have time-continuous (AC or else) solutions. Well-posedness of (2.16) is proved in [524] under passivity-like conditions, in both cases of AC and LBV solutions, hence allowing jumps, and relying on [368, Theorem 3]: this implies to re-interpret (2.16) as an MDI, being $\dot{x}$ a measure, in a similar way as done for the FOSwP (see (2.3) and (2.4)). A more general case is studied in [64, Theorem 4.4] with $\mathcal{F}(t, x)=\mathcal{N}_{\mathcal{S}(t)}(x)$ and $f(t, x)=H(t)(x)$ for some maximal monotone set-valued operator $H(\cdot), \operatorname{dom}(H(t)) \subset \mathcal{S}(t)$ for each $t, \mathcal{S}(\cdot)$ closed and AC set-valued mapping. As alluded to above, one important class of such DIs is when $\mathcal{F}(x)=\partial \varphi(x)$ with $\varphi(\cdot)$ proper, convex LSC [100]. Relaxing the convexity to other classes of functions (for instance, prox-regular functions), is worth investigating. This has been done mainly for sweeping process-like DIs, i.e., $\varphi(x)=\psi_{K}(x) \Rightarrow \partial \varphi(x)=\mathcal{N}_{K}(x)$, with $K \subseteq \mathbb{R}^{n}$ a prox-regular set $[238,415,414,105]$ (in this case the DI $(2.16)$ is sometimes called a deterministic Skorokhod problem [105]). In [338, section 7], the DI (2.16) is analysed with $\mathcal{F}(t, x)=\partial \varphi(x), \varphi(\cdot)$ Lipschitz (hence $\partial=\partial_{C}$ is Clarke's subgradient, see Section A.2.1), and $f(t, x)$ set-valued upper semicontinuous (see Appendix E). Same "subdifferential" DIs were previously studied in [410] with $f(\cdot)$ proper LSC [410, Proposition 3.1] or primal lower nice [410, Theorem 3.2 $]^{2}$. Interestingly, it is shown in [31] [33, Corollary 4.1], that a DI as (2.16) with $\mathcal{F}(t, x)=\mathcal{F}(x)$ maximal monotone, $f(t, x)=f(x)+\frac{m}{r} x, m>0$, is equivalent to the DI with $\mathcal{F}(t, x)=\mathcal{N}_{\mathcal{S}}(x)$, $f(t, x)=f(x)$, with $\mathcal{S}$ an $r$-prox-regular set (the maximal monotone operator exists which satisfies $\mathcal{N}_{\mathcal{S}} \cap \mathbb{B}(0, m)+\frac{m}{r} x \subset \mathcal{F}(x) \subset \mathcal{N}_{\mathcal{S}}+\frac{m}{r} x$ for all $\left.x \in \mathcal{S}\right)$. Applications exist with $\varphi(\cdot)$ regular [254]. Time-delays in $f(t, x)$ are taken into account in [318]. Further extensions of (2.16) are proposed in [154], as:

$$
\left\{\begin{array}{l}
E \dot{x}=A x+B \lambda \\
\lambda \in-\mathcal{M}(C x+D \lambda)
\end{array}\right.
$$

where $\mathcal{M}: \mathbb{R}^{n} \rightrightarrows \mathbb{R}^{n}$ is a maximal monotone operator, $E$ is a singular matrix. Under a suitable passivity assumption on the matrix tuple $(E, A, B, C, D)$ [130, Section 3.1.7], this system can be equally described by an inclusion $P \dot{x} \in-\overline{\mathcal{M}}(x)$ for some matrix $P \succcurlyeq 0$, and maximal monotone operator $\overline{\mathcal{M}}(\cdot)$. Lagrangian systems with singular mass matrix and bilateral (holonomic) constraints, unilateral constraints [137], also fall into this class. Quite similar singular DIs are studied in [245]. Notice that the system (2.17) could be rewritten as a set-valued input/output system:

$$
\left\{\begin{array}{l}
\dot{x} \in\left(A-B\left(D+\mathcal{M}^{-1}\right)^{-1} C\right)(x)+B r(t) \\
z \in\left(C-D\left(\left(D+\mathcal{M}^{-1}\right)^{-1} C\right)(x)\right.
\end{array}\right.
$$

where $r(\cdot)$ is a reference signal, and one wants to analyse the operator $r \mapsto z$. One may also consider a measured output $y=E x+F \lambda \in\left(E-F\left(\left(D+\mathcal{M}^{-1}\right)^{-1} C\right)(x)\right.$

\footnotetext{
${ }^{2}$ Primal lower nice functions have subdifferentials -Fréchet, Clarke, proximal or Mordukhovich are equal- which satisfy some hypomonotonicity property.
} 
(in some applications like circuits, see Section 4.1, $\lambda$ may be a physical quantity). Thus (2.17) gives rise to a family of set-valued I/O systems.

Further extensions of (2.16) are considered in [557] for hysteresis applications, and are known as double inclusions: $\left(\partial \psi_{K_{1}}\right)^{-1}(\dot{x})+\partial \psi_{K_{2}}(x) \ni u(t)$, or $\left(\partial \psi_{K_{1}}\right)^{-1}(\dot{x})+\partial \varphi(x) \ni$ $u(t), K_{1}$ and $K_{2}$ closed convex sets, $\varphi(\cdot)$ proper convex LSC function.

2.3. Differential variational inequalities (DVI). Another class of mathematical models which could be embedded in the framework of (1.1) are the differential variational inequalities (DVIs). They comprise an ODE and a Variational Inequality (VI), and were introduced in [462] and subsequently analysed and used in $[520,527,461,388,420,347,462,298,463,389,395,394,564,565,566]$. By definition, for a given mapping $\Psi(\cdot)$, and a set $K$, we say that $\lambda$ solves $\operatorname{VI}(K, \Psi)$, denoted $\lambda \in \operatorname{VI}(K, \Psi)$, if $\lambda \in K$ satisfies

$$
\left\langle\lambda^{\prime}-\lambda, \Psi(\lambda)\right\rangle \geq 0, \quad \forall \lambda^{\prime} \in K .
$$

For the system class DVI, we are basically interested in studying the models where the nonsmooth behavior results from the solution of a VI, and are described as [462]

$$
\left\{\begin{array}{l}
\dot{x}=f(t, x)+G(t, x) w \\
w \in \operatorname{VI}(K, \Psi(t, x, \cdot))
\end{array}\right.
$$

and evidently, in comparison with $(1.1)$, we have $\mathcal{F}(t, x, w)=-\mathrm{VI}(K, \Psi(t, x, \cdot))$. The reason for choosing a vector field in (2.21a) which is affine in $w$, instead of a general nonlinear function in $w$, is because as we increase the index of the DVI, $w$ would be a distribution in general, and nonlinearities in $w$ would not be well-defined mathematical objects in that case. The regularity of the solution to a DVI $(2.21)$ is determined by its index [520]. Under certain assumptions on the map $\Psi(t, x, \cdot)$, the static VI in (2.21b) has a solution $w$ and the map $(t, x) \mapsto w(t, x)$ may be a single-valued Lipschitz function. Indeed using (2.20) and the normal cone definition (see (A.2)), the static VI is rewritten equivalently as the generalized equation $0 \in \Psi(t, x, w)+\mathcal{N}_{K}(w)$. Then $w$ can be replaced in (2.21a) by a single-valued function of $x$, resulting in an ODE: this corresponds to the index 0 case. In case of index 1 DVIs, the mapping $\Psi(\cdot)$ is independent of $w$, and one needs to compute the derivative of $\Psi(\cdot)$ to solve for $w$. In such a case, the selection $w$ is a possibly discontinuous function. The notion of index in DVIs was introduced in [520] and is very close to the notion of relative degree in complementarity systems (Section 2.4) and in HOSwP (Section 2.1.3).

REMARK 2. In [462] it is mainly the case of index 0 that is analysed. This makes the DVIs in [462], depart from the DVIs analysed in [62, Chapter 5, §6] which read: $\langle\dot{x}-f(t, x), x-y\rangle \leq 0$ for all $y \in \mathcal{S}, \mathcal{S}$ a closed convex set, $x(t) \in \mathcal{S}$ for all $t$. This in turn is equivalent to: $\dot{x}-f(t, x) \in-\mathcal{N}_{\mathcal{S}}(x), x(0) \in \mathcal{S}$. Letting $\Psi(t, x, w)=w$ in (2.21), $K$ being a closed convex cone, and using (B.1) allows one to recover these DVIs from (2.20). The DVIs in [62, Chapter 5, §6] are therefore closer to the maximal monotone DIs of Section 2.2, see however Section 3.1 for more comments.

The results in [462] essentially rely on convexity of the time-invariant set $K$, with $\Psi(t, x, w)=F(w)+B(t, x), F(\cdot)$ continuous, $B(t, x)$ and $G(t, x)$ are Lipschitz continuous, $G(t, x)$ is bounded. Convexity is relaxed to prox-regularity in [293, Section 4] $[238,414,415]$ for other classes of DVIs (in [293], $K=K(t, x)$ ). However [462] consider Lipschitz continuous mappings $G(t, x)$, while [293, 415] essentially assume $G(t, x)=I$, one exception being generalized FOSwP [143, 338], which involve the term $g(x) \mathcal{N}_{\mathcal{S}(t)}(h(x))$ in the right-hand side, with various regularity conditions on 
both $g(\cdot)$ and $h(\cdot)[143$, Section 4] [338, Section 4]. Constant time delays in the vector field (2.21a) are considered in [566]. DVIs of the second kind are considered in [283, 14, 389, 395, 394], see Section 3.1 for more details. Other "natural" extensions of DVIs consist of replacing the classical VI in (2.20) by other kinds of VIs like hemi-VI [390], quasi-VI (this is the case of the SOSwP and HOSwP), see Section A.2.2 for definitions, or to consider fractional order derivatives in (2.21a) [396, 347, 420].

2.4. Complementarity systems. Complementarity conditions in the finitedimensional setting of Lagrangian dynamics have been introduced by J.J. Moreau in [432, 433], where he used Convex Analysis tools to analyze the extension of Gauss' principle to mechanical systems subjected to unilateral constraints.

2.4.1. Linear Complementarity Systems (LCS). So-called LCS have been introduced formally in [548] and analyzed subsequently in [304, 151, 307, 143]. They take the form:

$$
\left\{\begin{array}{l}
\dot{x}=A x+B \lambda+E_{1} u+E_{2} \\
0 \leq \lambda \perp z=C x+D \lambda+E_{3} u+E_{4} \geq 0 \\
\text { State jump rule }
\end{array}\right.
$$

where $u: \mathbb{R}_{+} \rightarrow \mathbb{R}^{p}$ is a control input, $z$ and $\lambda$ are complementary $m$-vector slack variables. Clearly (2.22) is not a linear system: it is strongly nonlinear and nonsmooth (one can interpret (2.22b) as a sort of nonsmooth constraint on $x$, see Section 3.12). Recalling (1.1), the set-valued mapping $\mathcal{F}(t, H x, J w)$ is represented implicitly by the complementarity conditions in (2.22b), with $w=\lambda, G(t, x)=B$, and $J=D$. An important parameter in the description of the LCS is the relative degree $r$ between $\lambda$ (seen as an input) and $z$ (seen as an output), or the index of the transfer matrix $D+C\left(s I_{n}-A\right)^{-1} B$ [306] (see Appendix C for definitions). As we shall see later, this is related to the order of the sweeping process and essentially determines the degree of the solution seen as a distribution. Obviously $r$ can take arbitrary values $\leq n$ in (2.22), similarly as for the HOSwP in Section 2.1.3. Let us make a qualitative description of the influence of $r$ on the LCS' dynamics, in case $m \geq 1$. If $r=0$, the state $x$ is unconstrained (the initial condition can be chosen in $\left.\mathbb{R}^{n}\right)$, and $\lambda \in \operatorname{SOL}(\operatorname{LCP}(C x+$ $\left.E_{3} u+E_{4}, D\right)$ ) (see Definition B.1). If $r \geq 1$, then $z=C x+E_{3} u+E_{4}$, hence $x \in \Phi=\left\{w \in \mathbb{R}^{n} \mid C w+E_{3} u+E_{4} \geq 0\right\}$ : the state lives in a convex closed polyhedral set. A closer analysis shows that when $r=1$ and $C B \succ 0$, then a bounded $\lambda(t)$ is always sufficient to keep the state in $\Phi$, provided that $x(0) \in \Phi$ and $u(\cdot)$ is continuous ${ }^{3}$. When $r \geq 2$, state re-initializations are necessary to render $\Phi$ invariant, independently of $u$. Hence the presence of (2.22c), that we will study in more details in Section 2.4.4. Let us now analyse what happens on the constraint boundary. Suppose that $z(t)=0$ for all $t \in\left[t_{0}, t_{1}\right], t_{1}>t_{0}$; then for each $t \in\left(t_{0}, t_{1}\right)$, assuming the right limit exists at $t$, one has $\left.\lambda\left(t^{+}\right) \in \operatorname{SOL}(\operatorname{LCP}(\underbrace{C A^{r} x(t)+E_{3} u^{(r)}+\sum_{i=1}^{r-1} C A^{i} E_{1} u^{(r-1+i)}\left(t^{+}\right.}_{\triangleq_{q_{r}}\left(x, \mathbf{u}\left(t^{+}\right)\right)}), \underbrace{C A^{r-1} B}_{\triangleq_{M_{r}}})\right)$, where $\mathbf{u}=\left(u, \dot{u}, \ddot{u}, \ldots, u^{(r)}\right)$ (we chose $E_{2}=0$ to simplify the expression). The LCP may be named the contact $L C P\left(q_{r}(x, \mathbf{u}), M_{r}\right)$, stemming from $0 \leq \lambda\left(t^{+}\right) \perp z^{(r)}\left(t^{+}\right) \geq$ 0 .

\footnotetext{
${ }^{3}$ State jumps, if they exist, are due to discontinuities in $u(\cdot)$.
} 
$\rightsquigarrow$ In LCS the leading Markov parameter (or decoupling matrix) has to be more than non singular: complementarity requires that it has to satisfy some positivity constraints (P-matrix, copositivity).

Thus let us assume that $M_{r}$ is a P-matrix. Another feature of LCS, lies in the following. Assume again that the system evolves on the constraint boundary, that is $z(t)=0$ for every ${ }^{4} t \in\left[t_{0}, t_{1}\right], t_{1}>t_{0}$. Whether or not $z(t)$ becomes positive in a right-neighborhood of $t_{1}$, can be tested through the lexicographical inequality $\left(z\left(t_{1}\right), \dot{z}\left(t_{1}^{+}\right), \ddot{z}\left(t_{1}^{+}\right), \ldots, z^{(i)}\left(t_{1}^{+}\right), \ldots\right) \succcurlyeq 0$ (see Appendix E). On such an interval, however, $\lambda(t)$ is the solution of the contact $\operatorname{LCP}\left(q_{r}(x, \mathbf{u}(t)), M_{r}\right)$. Assume for simplicity that $m=1$. (i) If $q_{r}\left(x\left(t_{1}\right), \mathbf{u}\left(t_{1}^{+}\right)\right)>0$ then $\lambda\left(t_{1}^{+}\right)=0$ and $z^{(r)}\left(t_{1}^{+}\right)>0$ : the system quits the constraint boundary in a right-neighborhood of $t_{1}$. (ii) If $q_{r}\left(x\left(t_{1}\right), \mathbf{u}\left(t_{1}^{+}\right)\right)<0$ then $\lambda\left(t_{1}^{+}\right)>0$ and $z^{(r)}\left(t_{1}^{+}\right)=0$ : the system stays on the constraint boundary in a right-neighborhood of $t_{1}$. (iii) If $q_{r}\left(x\left(t_{1}\right), \mathbf{u}\left(t_{1}^{+}\right)\right)=0$ then $\lambda\left(t_{1}^{+}\right)=0$ and $z^{(r)}\left(t_{1}^{+}\right)=0$ : this is the degenerated mode of the contact LCP, which is more delicate to analyse. One way to tackle this issue is to consider the canonical form in (2.8a)-(2.8c) extended to the case with non-zero $u(\cdot)$ (see also Appendix C) and the higher order derivatives of the "output" $z_{1}=z$. Let us assume that all variables, including $u(\cdot)$, are analytic on $\left(t_{0}, t_{1}\right)$ and also in the right-neighborhood of $t_{1}$. Using the fact that $z_{1}(t)=0$ on $\left(t_{0}, t_{1}\right)$ implies $z_{1}^{(i)}(t)=0$ for all $i \geq 1$ and all $t \in\left(t_{0}, t_{1}\right)$, we obtain that $q_{r}(x, \mathbf{u})=\bar{A} \zeta+\bar{B} \mathbf{u}$, and $\dot{\zeta}=A_{0} \zeta+G_{0} \mathbf{u}$, for some $\bar{A}, \bar{B}, G_{0}$, and $A_{0}$ is in (2.8c). Therefore $z_{1}^{(r+i)}\left(t^{+}\right)=\bar{A} A_{0}^{i} \zeta(t)+\bar{B} \mathbf{u}^{(i)}\left(t^{+}\right)+\sum_{j=0}^{i-1} \bar{A} A_{0}^{j} \mathbf{u}^{(i-j-1)}+M_{r} \lambda^{(i)}(t)$, $i \geq 0$. If $\lambda^{(j)}\left(t_{1}^{+}\right)=0$ and $z_{1}^{(r+j)}=0$ for all $j<i$, one can form the contact $\operatorname{LCP}\left(q_{r}^{i}\left(\zeta, \mathbf{u}^{(i)}\right), M_{r}\right): 0 \leq z_{1}^{(r+i)}\left(t^{+}\right) \perp \lambda^{(i)}\left(t^{+}\right) \geq 0$ and compute its solution. One can proceed iteratively starting with $i=1$ until one finds an $i$ such that $\lambda^{(r+i)}\left(t_{1}^{+}\right)>0$ or $z_{1}^{(r+i)}\left(t_{1}^{+}\right)>0$. Otherwise, if such an index does not exist, all contact LCPs are degenerated and the systems continues to "graze" the constraint boundary.

$\rightsquigarrow$ The crucial fact here is that all the contact LCPs hence built have the matrix $M_{r}$. If $M_{r}$ is a P-matrix, they all are well-posed. Another feature is that even if $u(t)=0$ for all $t$, the zero dynamics state $\zeta$ plays a specific role in the system's behaviour.

This shows that LCS are different from systems with "virtual" unilateral constraints which have to be respected using a control action $u(\cdot)$ solely [528]. In LCS with $r \geq 1$, $u(\cdot)$ can be used to control the system inside $\Phi$, but the invariance of $\Phi$ is guaranteed by the multiplier $\lambda$, which is in general a distribution.

REMARK 3. The above analysis leads naturally to so-called dynamical complementarity problems (not to be confused with dynamical complementarity systems) used in [549, 398]. What we described is a way to find a solution to: $\left(z_{1}, \dot{z}_{1}, \ldots, z_{1}^{(i)}, \ldots,\right)=0$ and $\left(\lambda, \dot{\lambda}, \ldots, \lambda^{(i)}, \ldots\right) \succ 0$ (sustained contact), or $\left(z_{1}, \dot{z}_{1}, \ldots, z_{1}^{(i)}, \ldots,\right) \succ 0$ and $\left(\lambda, \ldots, \lambda^{(i)}, \ldots\right)=0$ (detachment), or $\left(z_{1}, \ldots, z_{1}^{(i)}, \ldots,\right)=0$ and $\left(\lambda, \ldots, \lambda^{(i)}, \ldots\right)=0$ (grazing contact). Lexicographical inequalities are ubiquitous in the analysis of systems which evolve on the boundary of some domain of their state space [6, 528, 532, 569]. They are closely related to the so-called semi-observability cones $\{x \in$ $\left.\mathbb{R}^{n} \mid\left(C x, C A x, \ldots, C A^{n-1} x\right) \succcurlyeq 0\right\}[461,156]$, which stem from the observation that $\left(z, \dot{z}, \ldots, z^{(n)}\right)=\left(C x, C \dot{x}, \ldots, C x^{(n)}\right) \succcurlyeq 0$, and are used to state the (local) invariance of conic cells [156, Lemma 2.3].

REMARK 4. Since we have assumed analycity in a right-neighborhood of $t_{1}$, we could also carry out the analysis using Taylor expansions of $z_{1}(t)$ and of $\lambda(t)$. From

\footnotetext{
${ }^{4}$ It is assumed that solutions have left and right limits everywhere.
} 
(2.22b) and letting $h=t-t_{1}>0$ it follows that:

$0 \leq z(t)=z\left(t_{1}\right)+h \dot{z}\left(t_{1}^{+}\right)+\frac{h^{2}}{2} \ddot{z}\left(t_{1}^{+}\right)+\ldots \perp \lambda(t)=\lambda\left(t_{1}^{+}\right)+h \dot{\lambda}\left(t_{1}^{+}\right)+\frac{h^{2}}{2} \ddot{\lambda}\left(t_{1}^{+}\right)+\ldots \geq 0$

For $h$ small enough both inequalities imply lexicographical inequalities as the above ones.

In general an LCS as in (2.22) is a set-valued system. Exceptions are if the fundamental operator $x \mapsto\left(D+\mathcal{N}_{\mathbb{R}_{+}^{m}}\right)^{-1}\left(C x+E_{3} u+E_{4}\right)$, obtained from (2.22a) (2.22b), using (B.1) and the material in Section A.2.4, is single valued (see conditions in [135, Propositions 1, 2, 3, Corollary 1] [136] and [26, Lemma 3.4] which guarantee that this operator is single-valued Lipschitz continuous, under positive-definitenesslike conditions on $D)$, or if the set $B \operatorname{SOL}\left(\operatorname{LCP}\left(K, C x+E_{3} u+E_{4}, D\right)\right)=B \lambda$ with $\lambda \in-\left(D+\mathcal{N}_{\mathbb{R}_{-}^{m}}\right)^{-1}\left(C x+E_{3} u+E_{4}\right)$, is a singleton [156, 240]. The basic consistency condition for this operator to make sense, is $\operatorname{Im}\left(C+E_{3} u(t)+E_{4}\right) \cap \operatorname{Im}\left(\left(D+\mathcal{N}_{\mathbb{R}_{+}^{m}}\right)\right) \neq \emptyset$, using the fact that $\operatorname{dom}\left(\left(D+\mathcal{N}_{\mathbb{R}_{+}^{m}}\right)^{-1}\right)=\operatorname{Im}\left(D+\mathcal{N}_{\mathbb{R}_{+}^{m}}\right)$. In case of $(2.22)$, a sufficient condition for the single-valuedness is that $D$ is a P-matrix $(\Rightarrow r=0$, see Appendix C and Theorem B.3), so that the LCP in (2.22) (b) has for any $C x+E_{3} u+E_{4}$ a unique solution that is Lipschitz continuous in $C x+E_{3} u+E_{4}$ [210, Theorem 7.3.10]. In this case (2.22) (a) (b) is an ODE with Lipschitz continuous right-hand side, hence it is well-posed. In fact the well-posedness with $C^{1}$ solutions holds if and only if $B \operatorname{SOL}\left(\operatorname{LCP}\left(C x+E_{3} u+E_{4}, D\right)\right)$ is a singleton [462, Proposition 5.1] [157]: this is a very interesting point, that the well-posedness of the static feedback loop term in (1.1), implies the well-posedness of the whole dynamics. The case $D=0$ and $C B$ is a strict semicopositive matrix (see Theorem B.4), is studied in [510]. In case $r \geq 1$, solutions gets less regular and may be AC if $r=1$, or Bohl distributions if $r \geq 2$ [307] (which are a particular case of the Schwartz' distributions constructed in [6] for the HOSwP in Section 2.1.3). In such a case (2.22a) has to be rewritten as an equality of distributions (in a similar way to the HOSwP in Section 2.1.3), and (2.22b) has to be given a meaning at the state jumps.

2.4.2. Nonlinear Complementarity Systems (NLCS). A class of NLCS is introduced as:

$$
\left\{\begin{array}{l}
\dot{x}=f(x, \lambda, u, t) \\
0 \leq \lambda \perp z=Z(x, \lambda, u, t) \geq 0 \\
\text { State jump rule. }
\end{array}\right.
$$

We have $\mathcal{F}(t, H x, J w)=\mathcal{N}_{\mathbb{R}_{+}^{m}}(z), w=\lambda$. The case $f(x, \lambda, u, t)=f(x)+g(x) \lambda$, $Z(x, \lambda, u, t)=h(x), f(\cdot), g(\cdot), h(\cdot)$ smooth vector fields, is studied in [549], with local existence and uniqueness of smooth solutions. See also [462, Theorem 5.2] for local existence of smooth solutions, under a relative degree 0 condition. The case $f(x, \lambda, u, t)=a(x)+B \lambda+e(x, u), Z(x, \lambda, u, t)=c(x)+g(u), a(\cdot), e(\cdot)$ continuous, $g(\cdot)$ locally Lipschitz, $c(x)=B \nabla V(x)$ for some $V \in \mathcal{C}^{3}\left(\mathbb{R}^{n} ; \mathbb{R}_{+}\right)$, is studied in [143], with global existence and uniqueness of AC or RCLBV solutions. The uniform relative degree between the "input" $\lambda$ and the "output" $z$ (when it exists) is once again shown to play a crucial role in the system's dynamics. Passivity-like conditions of the form $c^{T}(x)=\frac{\partial V}{\partial x}(x) B$ for some function $V \in \mathcal{C}^{3}\left(\mathbb{R}^{n} ; \mathbb{R}^{+}\right)$with Hessian $\frac{\partial^{2} V}{\partial x^{2}}(x) \succ 0$, allow to recover a FOSwP with a prox-regular set $\mathcal{S}(t)$ [143]. This is extended in [338, Theorems 9.3, 9.5] where some regularity assumptions are relaxed, global existence of 
AC solutions is shown, and $f(x, \lambda, u, t)=f(t, x)+g(x) \lambda$, while $(2.24 \mathrm{~b})$ is extended to a CCP: $K^{\star} \ni \lambda \perp z=h(x)+d(t) \in K, K$ a closed convex cone. Optimal control formulations are used to numerically solve some classes of NLCS in [576], using Fischer-Burmeister complementarity functions to design the objective function. One important example of NLCS are complementarity Lagrangian systems, see Section 4.2.1. NLCS have the intrinsic mathematical difficulty that products of distributions are not defined, except in very particular cases. This certainly puts strong restrictions on the classes of NLCS which can be studied.

2.4.3. Perspectives and Extensions. Let $K \subseteq \mathbb{R}^{m}$ be a non empty convex cone, the complementarity conditions in $(2.22 \mathrm{~b})$ can be extended to a linear cone complementarity problem (LCCP) as: $K^{\star} \ni \lambda \perp z=C x+D \lambda+E_{3} u+E_{4} \in K$, where $K^{\star}$ is the dual cone, yielding a Linear Cone Complementarity System (LCCS) [157]. The LCCP defines a set-valued operator, and we have $\mathcal{F}(t, H x, J w)=\partial \psi_{K}(z), w=\lambda$. Going a step further, $K$ being a closed convex cone, $\lambda \in-\partial \psi_{K}(z) \Leftrightarrow z \in \mathcal{N}_{K^{\circ}}(-\lambda)$, hence $C x+E_{3} u+E_{4} \in\left(D+\mathcal{N}_{K^{\circ}}\right)(-\lambda) \Leftrightarrow \lambda \in-\left(D+\mathcal{N}_{K^{\circ}}\right)^{-1}\left(C x+E_{3} u+E_{4}\right)$, which provides an alternative form for $\mathcal{F}(t, H x, J w)$. Note that in (1.1), the setvalued element $w$ can be described by $w \in\left(I_{m}+\mathcal{F}(t, H x, J w)\right)^{-1}(0)$, and in case of LCCS we have $\left(I_{m}+\mathcal{F}(t, H x, J w)\right)^{-1}(0)=\left(I_{m}+\mathcal{N}_{K}\left(C x+E_{3} u+E_{4}+D^{\cdot}\right)\right)^{-1}(0)=$ $\left(D+\mathcal{N}_{K^{\star}}\right)^{-1}\left(-C x-E_{3} u-E_{4}\right)=-\left(D+\mathcal{N}_{K^{\circ}}\right)^{-1}\left(C x+E_{3} u+E_{4}\right)$. Just like LCS, an LCCS is therefore fundamentally a set-valued system, except for particular cases. The basic idea in $[135,136]$ is as follows: since an LCCS can be viewed as an LTI system with a set-valued mapping $-w \in \partial \psi_{K}(C x+D \lambda+G u+H)$ for some closed convex cone $K$, in negative feedback, we may consider mappings $-w \in \partial \varphi\left(C x+D \lambda+E_{3} u+E_{4}\right)$ with $\varphi(\cdot)$ a convex, LSC proper function (in $(2.22)$ we have $\varphi=\partial \psi_{\mathbb{R}_{+}^{m}}$, or $\varphi=\partial \psi_{K}$ ). Thus $\mathcal{F}(t, H x, J w)=\partial \varphi\left(C x+D \lambda+E_{3} u+E_{4}\right)$. Proceeding similarly as for LCS, we get $\lambda \in-\partial \varphi(z) \Leftrightarrow z \in \partial \varphi^{\star}(-\lambda)$, consequently $C x+E_{3} u+E_{4} \in\left(D+\partial \varphi^{\star}\right)(-\lambda) \Leftrightarrow \lambda \in$ $-\left(D+\partial \varphi^{\star}\right)^{-1}\left(C x+E_{3} u+E_{4}\right)$. Hence $\mathcal{F}(t, H x, J w)=\left(D+\partial \varphi^{\star}\right)^{-1}\left(C x+E_{3} u+E_{4}\right)$. Depending on $\varphi(\cdot), D, C, E_{3}$ and $E_{4}$, this may be equivalent to a FOSwP, or to a maximal monotone DI as in (2.16), or to an ODE with Lipschitz right-hand side. We note that LCS can also be seen as switching DAEs, where the switches are state dependent and ruled by complementarity conditions. Further extension is in [159] where a general maximal monotone operator (not necessarily the subdifferential of a convex LSC function) is considered. Possible future generalizations could be to replace convex functions $\varphi(\cdot)$ by prox-regular functions [492], as done for FOSwP in $[204,238,530,143]$. One important issue lies in the fact that in the non convex case, the biconjugate $\left(\varphi^{\star}\right)^{\star}$ may not equal $\varphi$, so that manipulations used to invert the set-valued part may no longer be valid (one path could be to use the results in [31]). Other extensions which have not been tackled, could be: consider a mixed LCP instead of an LCP in (2.22) (b), yielding the MLCS:

$$
\left\{\begin{array}{l}
\dot{x}=A x+B_{1} \lambda_{1}+B_{2} \lambda_{2}+E_{1} u+E_{2} \\
A_{1} \lambda_{1}+A_{2} \lambda_{2}+E_{5} x+E_{6}=0 \\
0 \leq \lambda \perp z=C x+D_{1} \lambda_{1}+D_{2} \lambda_{2}+E_{3} u+E_{4} \geq 0 \\
\text { State jump rule. }
\end{array}\right.
$$

Inserting PWL characteristics in dynamical systems, yields such MLCS (see [125, Examples 4-7], and Section 3.15). If the linear equation in (2.25b) can be inverted for $\lambda_{1}$ or for $\lambda_{2}$, then we are back to (2.22). But the MLCP (2.25b), (2.25c) may have solutions without $(2.25 \mathrm{~b})$ being invertible. In any case, the notion of relative 
degree between $z$ and $\lambda$ for the dynamics (2.25a)-(2.25c) is unclear in general. Since LCPs are central in the analysis of LCS, it is expected that MLCPs and their wellposedness could be central for MLCS. It could be interesting to investigate the properties of particular dynamical structures (systems with various PWL interconnections: in sandwich, forward block, negative feedback, etc) and of classes of PWL mappings. However it is noteworthy that physical systems like circuits, are usually represented by a set of evolution equation and constraints, far more complex than (2.22) or even (2.25), see [2, Section 5.1]. As another extension one may consider that $C=C(u)$ in $\left(2.25 \mathrm{c}\right.$ ), which has a different effect on the state space partitioning than $E_{3} u$ (see Section 3.4). Getting back to LCS, open issues are in delay systems (as for FOSwP, the delay can act at different places: the vector field in (2.22a), $\lambda$ in (2.22a) or/and in (2.22b), $z$ in $(2.22 \mathrm{~b})$ ) (see [107] for preliminary results), time-varying LCS with $A(t), B(t), C(t), D(t)$ (see Section 3.4 for a possible path), and control-related issues (optimal control has been tackled in [287, 555, 554], yielding MPEC problems, with applications in process control $[484,96]$, trajectory tracking, etc). It could be of interest to study the relationships between optimal control of FOSwP tackled in $[54,160,162,161,200,202,201,205,316,217,536]$, which rely on the convergence of a suitable time-discretization or of a regularization of the normal cone, and the results in $[555,554]$, which rely on [287]. Further extensions are state-dependent switching LCS, and singular LCS (with $P \dot{x}$ in the left-hand side of (2.22a) or (2.25a) for a singular $P$, see also the circuit in (4.7) for more general $P$ ). Let us mention also a new kind of LCS defined in an implicit way, see Section 3.5.

EXAMPLE 2. Let us illustrate how MLCS (more generally $M N L C S$ ) arise naturally in so-called optimization-constrained differential equations [148, 373, 51], which take the form:

$$
\begin{aligned}
& \dot{x}=f(t, x)+b(z) \\
& z=\arg \min _{h_{1}(\tilde{z}, x) \geq 0} F(\tilde{z}) . \\
& h_{2}(\tilde{z}, x)=0
\end{aligned}
$$

In atmopsheric chemistry applications, $F(\cdot)$ is a non convex nonlinear objective function, $h_{1}(\cdot)$ is linear, $h_{2}(\cdot)$ is nonlinear [373]. Using the KKT necessary conditions, it follows that there exists two multipliers $\lambda_{1}$ and $\lambda_{2}$ such that:

$$
\begin{aligned}
& \dot{x}=f(t, x)+b(z) \\
& \nabla F(z)-\nabla h_{1}(z, x) \lambda_{1}+\nabla h_{2}(z, x) \lambda_{2}=0 \\
& 0 \leq \lambda_{1} \perp h_{1}(z, x) \geq 0, \quad h_{2}(z, x)=0 .
\end{aligned}
$$

where $\nabla h_{1}(z, x)=\frac{\partial h_{1}}{\partial z}(z, x)^{T}$ and $\nabla h_{2}(z, x)=\frac{\partial h_{2}}{\partial z}(z, x)^{T}$. Let now $b(z)=B z$, $h_{1}(\tilde{z}, x)=H_{1} \tilde{z}+\bar{H}_{1} x, h_{2}(\tilde{z}, x)=H_{2} \tilde{z}+\bar{H}_{2} x, f(t, x)=A x$, and $F(\tilde{z})=\frac{1}{2} \tilde{z}^{T} M \tilde{z}$, $M=M^{T} \succ 0$. Then (2.27) becomes:

$$
\begin{aligned}
& \dot{x}=A x+B M^{-1} H_{1} \lambda_{1}-B M^{-1} H_{2} \lambda_{2} \\
& 0 \leq \lambda_{1} \perp H_{1} M^{-1} H_{1}^{T} \lambda_{1}-H_{1} M^{-1} H_{2}^{T} \lambda_{2}+\bar{H}_{1} x \geq 0 \\
& H_{2} M^{-1} H_{1}^{T} \lambda_{1}-H_{2} M^{-1} H_{2}^{T} \lambda_{2}+\bar{H}_{2} x=0 .
\end{aligned}
$$

The analogy with (2.25a)-(2.25c) is obvious. The MLNCS (2.27) is a nonlinear version.

2.4.4. State Discontinuities in LCS. It is noteworthy that the mathematical formalisms in (2.22a) (2.22b) and (2.24a) (2.24b) are meaningful only at times where the multiplier $\lambda$ and the variable $z$ are functions of time (in case $\lambda$ jumps, one may 
work with right limits). As alluded to above, there are cases where state jumps are necessary. Then $\dot{x}$ is a Schwarz' distribution and so are $\lambda$ and $z$. The SOSwP and HOSwP formalisms in (2.6) and (2.9) accomodate for such situations and are valid for all $t \geq 0$, being formulated in terms of measures or distributions. As explained in Section 2.1.4, they both include a suitable state jump rule. However in LCS one has to complete the dynamics with a suitable state jump which re-initializes, if needed, the state $x$ at times of switches between different modes of the complementarity conditions, enabling the integration in another mode. When a change of mode is detected, the state is projected onto the consistent subspace of the DAE corresponding to the new mode, see [307, Sections 4.2, 4.3, 4.4]. This is quite similar to what is done in exogenously switching DAEs [525, 391]. One additional analytical difficulty with LCS is however the mode selection issue [307, Section 5, Remark 6.11], that is not present in exogenously switching DAEs.

REMARK 5. We may make two comments about HOSwP and LCS. First, in the HOSwP the state jump rule automatically follows from a suitable projection on a convex set, without further calculations (because the dynamics has been put in a suitable form). Second the HOSwP chooses automatically a mode (and can thus be time-discretized with a suitable event-capturing time-stepping scheme [6, Section 5]). On the contrary the LCS is formulated with an event-driven philosophy (integrate until an event is detected, apply a state jump and start again the integration until another nonsmooth event is detected, see [307, Algorithm 7.1]). See Section 3.9 for more information on the relations between HOSwP and complementarity systems.

There is one important case where state jump rules can be explicitly given and calculated easily: dissipative LCS $[151,155]$, with main application in circuits (see Section 4.1). State jumps in passive electrical circuits with set-valued electronic components, and in dissipative LCS are analysed in [2, 143, 149, 151, 155, 256, 284].

DeFINITION 2.2. [284] Let us consider the dynamics in (2.22), and suppose that $(A, B, C, D)$ is passive in the sense of Definition D.1, with storage function $V(x)=$ $\frac{1}{2} x^{T} P x, P=P^{T} \succ 0$. For any $x\left(t^{-}\right)$, the state after the discontinuities, i.e., $x\left(t^{+}\right)$, is given by the solution of the generalized equation:

$$
P\left(x\left(t^{+}\right)-x\left(t^{-}\right)\right) \in-\mathcal{N}_{K}\left(x\left(t^{+}\right)\right)
$$

where $K \triangleq\left\{z \in \mathbb{R}^{n} \mid C z+E_{3} u\left(t^{+}\right)+E_{4} \in Q_{D}^{\star}\right\}$, with $Q_{D}^{\star}$ the dual cone of $Q_{D}=$ $\left\{z \in \mathbb{R}^{m} \mid z \geq 0, D z \geq 0, z^{T} D z=0\right\}$. Moreover the constraint qualification (CQ) $E_{3} u(t)+E_{4} \in Q_{D}^{\star}+\operatorname{Im}(C)$ holds.

Notice that $Q_{D}$ is a closed convex cone, and that $K$ is a polyhedron for each $t$. The similarity between (2.29) and (2.12) is clear. The CQ guarantees that the LCP: $0 \leq \lambda \perp w=C x+D \lambda+E_{3} u+E_{4} \geq 0$, has a solution whenever $D \succcurlyeq 0$. It also implies that $K$ is a convex cone. The inclusion in (2.29) is very close to that in (2.12): the relationships between LCS and FOSwP will be analysed further in Section 3.4. As we pointed out in Section 2.1.4, computing a projection (even on a convex set) may not be obvious in practice. The next lemma clarifies this.

Lemma 2.3. [143, 155, 284] Under the conditions of Definition 2.2, the following 
equivalences hold:

$$
\begin{gathered}
P\left(x\left(t^{+}\right)-x\left(t^{-}\right)\right) \in-\mathcal{N}_{K}\left(x\left(t^{+}\right)\right) \Longleftrightarrow \\
P\left(x\left(t^{+}\right)-x\left(t^{-}\right)\right)\left(x\left(t^{+}\right)-y\right) \geq 0, \quad \text { for all } y \in K \Longleftrightarrow \\
x\left(t^{+}\right)=\arg \min _{x \in K} \frac{1}{2}\left(x-x\left(t^{-}\right)\right)^{T} P\left(x-x\left(t^{-}\right)\right) \Longleftrightarrow \\
K \ni x\left(t^{+}\right) \perp P\left(x\left(t^{+}\right)-x\left(t^{-}\right)\right) \in K^{\star} \Longleftrightarrow \\
x\left(t^{+}\right)=\operatorname{proj}_{P}\left[K ; x\left(t^{-}\right)\right] \Longleftrightarrow\left\{\begin{array}{l}
P\left(x\left(t^{+}\right)-x\left(t^{-}\right)\right)=C^{T} \sigma \\
v=C x\left(t^{+}\right)+E_{3} u\left(t^{+}\right)+E_{4} \\
Q_{D}^{\star} \ni v \perp \sigma \in Q_{D}
\end{array} \Longleftrightarrow\right. \\
\left\{\begin{array}{l}
x\left(t^{+}\right)-x\left(t^{-}\right)=B \sigma \\
v=C x\left(t^{+}\right)+E_{3} u\left(t^{+}\right)+E_{4} \\
Q_{D}^{\star} \ni \nu \perp \sigma \in Q_{D} .
\end{array}\right. \\
\begin{array}{l}
v=C x\left(t^{-}\right)+E_{3} u\left(t^{+}\right)+E_{4}+C B \sigma \\
Q_{D}^{\star} \ni v \perp \sigma \in Q_{D} .
\end{array}
\end{gathered}
$$

Moreover, if the constraint qualification $E_{3} u(t)+E_{4} \in Q_{D}^{\star}+\operatorname{Im}(C)$ is satisfied, then the set $K \neq \emptyset$ and the projection is unique. Finally if $0 \in K$, the state jump law is dissipative since it secures $V\left(x\left(t^{+}\right)\right)-V\left(x\left(t^{-}\right)\right) \leq 0$.

Several formulations in (2.30) are good candidates for numerical computations. See Remark 11 for further informations about state jumps in LCS. Clearly the mappings in (2.30) are very close to the FOSwP mapping in (2.12), and could be extended along the lines of the HOSwP or [488].

2.5. Projected dynamical systems (PDS). This class of systems was apparently introduced in [310,311] and has been analysed afterwards in [62, 449, 232, 192, $327,584]$. Let $K \subseteq \mathbb{R}^{n}$ be a closed convex non empty set. A projected dynamical system (PDS) is given as one of the following equivalent formulations, with $f: \mathbb{R}^{n} \rightarrow \mathbb{R}^{n}$ and $g: \mathbb{R}_{+} \rightarrow \mathbb{R}^{n}$ two mesurable functions [133]:

(a) $\dot{x}=\operatorname{proj}\left[\mathcal{T}_{K}(x) ;-f(x)-g(t)\right]$

(b) $\dot{x}=\lim _{\delta \searrow 0} \frac{\operatorname{proj}[K ; x+\delta(-f(x)-g(t))]-x}{\delta}$

(c) $\dot{x}=-f(x)-g(t)-\operatorname{proj}\left[\mathcal{N}_{K}(x) ;-f(x)-g(t)\right]$

$(d) \dot{x}=-f(x)-g(t)-\langle\bar{v},-f(x)-g(t)\rangle \bar{v}$, with $\bar{v}=\frac{\operatorname{proj}\left[\mathcal{N}_{K}(x) ;-f(x)-g(t)\right]}{\left\|\operatorname{proj}\left[\mathcal{N}_{K}(x) ;-f(x)-g(t)\right]\right\|}$.

At this stage, equivalence just means that the four right-hand sides in (2.31) are identical: (2.31) (a) $\Leftrightarrow(2.31)$ (b) is proved in [313, Proposition III.5.3.5], (2.31) (a) $\Leftrightarrow(2.31)(\mathrm{c}),(2.31)(\mathrm{c}) \Leftrightarrow(2.31)$ (d) are proved in [133, Corollary 1]. Let us consider (2.31) (a). If $x \in \operatorname{Int}(K) \Rightarrow \mathcal{T}_{K}(x)=\mathbb{R}^{n}$, one gets $\dot{x}=-f(x)-g(t)$ : the system moves freely inside $K$. If $x \in \mathrm{bd}(K)$, then the vector field is projected onto the tangent cone, hence it points inside $K$ (see Figure 2.2 for an illustration in the plane). Let us illustrate further in a scalar case, the difference with a simple projection on a constant set. Let $K=\mathbb{R}_{+}$, then using (2.31) (a) and (A.1), we obtain $\dot{x}=-f(x)-g(t)$ if $x>0$, 
$\dot{x}=\max (0,-f(x)-g(t))$ if $x=0$. This is sometimes denoted $\dot{x}=[-f(x)-g(t)]_{x}^{+}$ [190, 244].

Let us denote the right-hand side of (2.31) (a) as $F(t, x)$. It is natural at this stage to make the link with Aubin's and Nagumo's viability theory [62], since by construction $F(t, x) \in \mathcal{T}_{K}(x)$ almost everywhere. Notice however that one projects onto $\mathcal{T}_{K}(x)$, not on $K$ itself: hence $F(t, x)$ may be discontinuous (as we shall see in Section 3.8, PDS are equivalent also to particular differential inclusions, and one should certainly better employ $\in$ rather than $=$ in (2.31), see [62, Equation (23) p.217]).

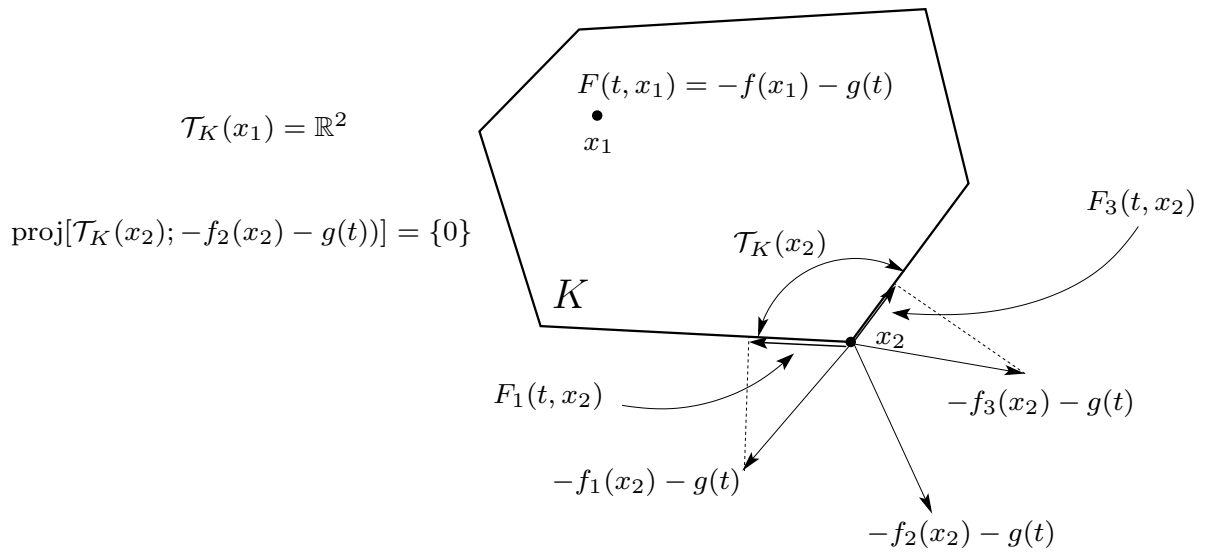

Fig. 2.2: Projected dynamical system in the plane.

REMARK 6. A set-valued upper semicontinuous, compact convex valued $f(x)$ is considered in [311]. The extension of (2.31) (a) to non convex sets $K$, using Clarke's tangent cone $\mathcal{T}_{K}^{C}(x)$ which is convex (see Section A.1.1), hence securing a unique projection, is doable. It is also possible to project in a non Euclidean metric [303]. The basic PDS in (2.31)(a), was generalized by replacing $f(x)$ with an upper semicontinuous set-valued mapping with non empty, compact and convex values $F$ : $K \rightrightarrows \mathbb{R}^{n}$ in [209], with $K$ tangentially regular set (see Section A.1.1).

Another class of projected systems is introduced in [106, 111], with $K=\left\{x \in \mathbb{R}^{n} \mid A x+\right.$ $b=0\}, \varphi(\cdot)$ and $\phi(\cdot)$ proper convex closed functions, $\epsilon(\cdot)$ is AC and decreasing, $\epsilon(t)>0$ for all $t \geq 0$, as:

$$
\dot{x} \in-x+\operatorname{proj}[K ; x-\partial \varphi(x)-\epsilon(t) \partial \phi(x)] .
$$

This is some kind of extension of the so-called global projective dynamics, defined as $[468,571,258,291]$ :

$$
\dot{x}=\operatorname{proj}[K ; x-\gamma f(x)]-x,
$$

for some single-valued function $f(\cdot), \gamma>0$ and $K$ a closed convex set. On the contrary the projected systems in (2.31) may be called local projective dynamics, since the tangent cone has a local nature.

2.6. Switching systems. Switching systems are widely studied in control systems, and may take various forms, some of which we review now. Roughly speaking, one splits the state space $\mathbb{R}^{n}$ into $p \leq+\infty$ closed cells $R_{i}$ such that: $\operatorname{int}\left(R_{i}\right) \cap \operatorname{int}\left(R_{j}\right)=$ 
$\emptyset$ for all $i \neq j, \cup_{1 \leq i \leq p} R_{i}=\mathbb{R}^{n}$. In each cell one assigns a vector field $f_{i}(x, t)$, and we define $\dot{x}=f(x, t)$, with $f(x, t)=f_{i}(x, t)$ if $x \in R_{i}, 1 \leq i \leq p$. If $x$ belongs to the boundary between several cells, in general $f_{i}(x, t) \neq f_{j}(x, t)$ for $i \neq j$ : the total vector field obtained by concatenating the vector fields $f_{i}(x, t)$ may be discontinuous at boundaries. When the vector fields $f_{i}(x, t)$ are linear, one speaks of a PWL system. The first issue is then to determine whether or not this switching system (a trajectory could a priori cross a boundary bd $\left(R_{i}\right)$ and enter another cell, or stay in the boundary between several cells (one then speaks of a sliding-motion)) is well-posed: usually the existence of solutions problem is expedited using Filippov's convexification method (though this is not the only way to deal with it, the complementarity approach being another one in some cases, see Section 3.11), however the uniqueness issue is far more constraining. Several approaches exist in the abundant literature, and many different descriptions of PWL systems exist [378, Chapter 2, Figure 2.9].

2.6.1. Filippov's differential inclusions. This type of DIs has been introduced in the context of robust sliding-mode control, that yields set-valued controllers $[248,249,542]$. It is a convexification of discontinuous vector fields, where the discontinuities may appear at submanifolds of codimension $\geq 1$ which correspond to the boundaries between the cells. A fundamental concept of Filippov's DIs is that of attractive sliding surfaces and sliding modes which play a significant role in robust feedback control. The general formulation of Filippov's set-valued right-hand side is:

$$
F(x, t)=\overline{\operatorname{conv}}\left\{\lim _{x^{\prime} \rightarrow x} f\left(x^{\prime}, t\right), t \text { is constant, }\left(x^{\prime}, t\right) \in \mathbb{R}^{n+1} \backslash\left(\cup_{1 \leq i \leq p} \operatorname{bd}\left(R_{i}\right)\right)\right\},
$$

where $\overline{\text { conv }}$ denotes the closure of the convex hull. An equivalent definition is given by $F(x, t)=\bigcap_{\epsilon>0, \operatorname{meas}(Z)=0} \overline{\operatorname{conv}}\{f(B(x, \epsilon) \backslash Z, t)\}$, with $f(B(x, \epsilon) \backslash Z, t)=\{f(y, t) \mid y \in$ $B(x, \epsilon) \backslash Z\}, B(x, \epsilon)$ is the ball of center $x$ and radius $\epsilon$. Thus Filippov's DI considers only the values of the vector fields $f_{i}(x)$ outside the switching surfaces, and takes the convex hull of these values when the system's state evolves on the switching surface. In $\operatorname{int}\left(R_{i}\right)$ it reduces to a single point $f(x, t)$, at the intersection of several boundaries $R_{i_{k}}, k=1, \ldots, p^{\prime} \leq p$, it is a segment, or a convex polygon, or a convex polyhedron [455, Section 2.2.1]. Therefore, Filippov's DIs differ from PWL systems studied in $[326,325]$ because they provide a model that "forgets" the values that the singlevalued vector fields may possess on the boundaries between the cells, and replaces it with a convex set. On the contrary PWL systems in [326, 325, 534] keep the value of each vector field $f_{i}(x)$ on the boundaries (this is a modeling step). Solutions of PWL systems will therefore be understood as Caratheodory solutions without leftaccumulations of switching times [266, Definition 12.1], and are different from AC solutions for Filippov's DI, or locally analytic solutions [480, Remark 2]. See [326, 325] and [266, Section 12] for well-posedness studies of PWL systems. Alternatives to Filippov's convexification of discontinuous vector fields, are Aizerman-Pyatnistskii [44, 45, 404, 405], Utkin [542] and Krasovskii [515] regularizations, that give rise to various types of DIs [249, Section 4]. Equivalency holds under some conditions [591, Theorem 14] [515, 317], but in general they do not provide the same right-hand sides [7].

REMARK 7. Most of Filippov's and PWL systems do not fall into the Lur'e class of systems analysed in this article, because their set-valued right-hand side $F(x)$ fails to define a maximal monotone operator (consequently in general Filippov's framework yields non unique solutions on codimension $\geq 2$ sliding surfaces). Few of them do, anyway (see Section 2.6.2). Also we shall see in Section 3.14 that they can be useful for the study of mechanical systems with a single unilateral constraint. 
$\rightsquigarrow$ It is noteworthy that both switching systems and Filippov's DIs (or other regularizations), are very general formulations for which it may not be always obvious to perform numerical analysis and simulations. This is one reason why it is important to examine when they can be equivalently rewritten as more tractable dynamical systems, like LCS or else for which powerful time-discretizations exist [7].

2.6.2. Ideal relay (signum) systems. Ideal relay (without hysteresis, may be named as well set-valued signum systems) are systems of the form [149, Chapter 7] [305]:

$$
\left\{\begin{array}{l}
\dot{x}=A x+B \lambda+E_{1} u \\
\lambda \in-\operatorname{Sgn}(z) \\
z=C x+D \lambda+E_{3} u+E_{4},
\end{array}\right.
$$

where $\operatorname{Sgn}(z)=\partial\left(\left|z_{1}\right|+\left|z_{2}\right|+\ldots+\left|z_{m}\right|\right), \partial$ is the subdifferential of convex analysis (see Section A.2.1), and we have $\lambda=w \in \mathbb{R}^{m}, z \in \mathbb{R}^{m}, \mathcal{F}(t, H x, J w)=\operatorname{Sgn}(C x+D \lambda$ ), $G(t, x)=B, H=C, J=D$. One sees that the signum set-valued function separates the state space into cells $R_{j}=\left\{x \in \mathbb{R}^{n} \mid z_{i}>0, i \in \mathcal{I}^{+}(u), z_{i}<0, i \in \mathcal{I}^{-}(u)\right\}$ for some index sets $\mathcal{I}^{+}(u)$ and $\mathcal{I}^{-}(u)$. The fundamental operator is thus equal to $\left(I_{m}+\operatorname{Sgn}\left(C x+E_{3} u+E_{4}+D^{\cdot}\right)\right)^{-1}(0)$, which can be expressed more explicitly as shown in Remark 12 later. The relative degree between $z$ and $\lambda$ happens to play once again a crucial role in the well-posedness (uniqueness of solutions) [480, 397]: relative degree $\geq 3$ may result in strange behaviours like infinity of AC solutions starting with reversed accumulation of switching instants (a phenomenon similar to what happens in mechanical systems with unilateral constraints and non analytic external forces [130, Section 2.4.3.1]). Positivity conditions (leading Markov parameter $C A^{r-1} B \succ 0$ [480], or the transfer matrix of $(A, B, C, D)$ being a P-matrix [397]) are crucial for the existence and uniqueness of so-called forward solutions (which are analytic solutions in a right-neighborhood of the initial time). As recalled in Remark 12, when $D=D^{T} \succ 0$ the relay system is an ODE with Lipschitz right-hand side. When $D=0,(2.35)$ becomes a DI whose right-hand side is compact convex for all $x$, and can be recast into Filippov's DIs (see Section 2.6.1). When $D \succcurlyeq 0$ one may assume a special block diagonal structure for $D$ (and use for instance [135]), or that the quadruple $(A, B, C, D)$ is passive in the sense of Definition D.1. Relay systems with a delay acting in the signum function or/and the vector field have been studied in [257, 451], where the set-valuedness plays no role, however. See Section 3.10 for further developments on relay systems.

The systems in (2.35) are linear in $\lambda$. There are other classes of relay systems, nonlinear in $\lambda$, as the ones studied in $[404,405,7]$ for gene regulatory networks models. These relay systems involve products of signum (or step) multifunctions.

2.6.3. Hysteresis Operators. Hysteresis operators are present in many physical systems and also in feedback control, see e.g., [27, 110, 360, 366, 558, 577], a classical reference being [557]. Among the earliest results on the stability of Lur'e systems with hysteresis feedback loop, let us mention the Russian school [80, 573, 574], more recently [359, 469, 335, 457]. There are different models of hysteresis, few of them depicted in Figure 2.3: (a) the square ( $y$ can move in both directions on the vertical segments, solid arrows) and the completed relay ( $y$ can move only in one direction on the reachable vertical segments, dashed arrows), (b) the play operator $((u, y)$ can move in both directions everywhere in the graph) [71], (c) a zig-zag operator [378], (d) and the relay hysteresis, or delayed relay operator [557, Section IV.1] 
(the system cannot stay on the dashed vertical branches, but jumps over them in one direction only). In cases (a) (b) (c), the couple $(u, y)$ can attain any point of the graph. The square and the zig-zag can be viewed as static set-valued operators $u \mapsto y$ (the simplest example may be the relay multifunction $y \in \operatorname{sgn}(u)$ of Section 2.6.2), or as examples of non monotone set-valued operators (hence there is no way to express these as subdifferentials of convex functions). However the derivative $\dot{u}$ usually comes into play in hysteretic phenomena [558], as in the Duhem hysteresis that writes down [335] [558, Section 1.2] [557, Chapter V] :

$$
\dot{y}=f_{1}(y, u) \max (0, \dot{u})+f_{2}(y, u) \min (0, \dot{u})
$$

where $f_{1}(\cdot), f_{2}(\cdot)$ and $u(\cdot)$ are continuously differentiable in both arguments, $u(\cdot)$ is AC. The hysteresis is a mapping $\left(u, y_{0}\right) \mapsto y=\Phi\left(u, y_{0}\right), y(0)=y_{0}$. Notice that choosing $f_{1}=0$ and $f_{2}=2$, and a suitable sequence of signs of $\dot{u}(\dot{u}>0$, then $\dot{u}<0$, then $\dot{u}>0$ ), one obtains zig-zag-like graphs in the $(u, y)$-plane. Vertical segments are however not possible since they would imply unbounded $f_{1}(\cdot)$ or $f_{2}(\cdot)$. Interestingly enough, Duhem operators possess dissipativity properties which can be

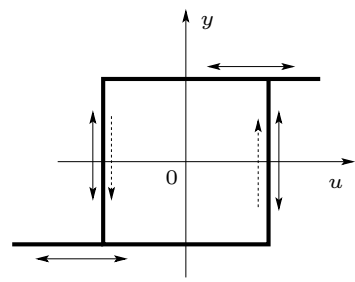

(a)

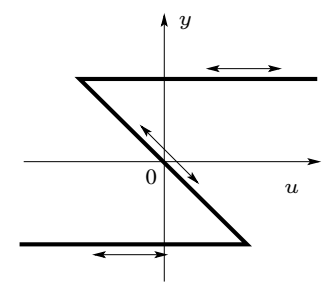

(b)

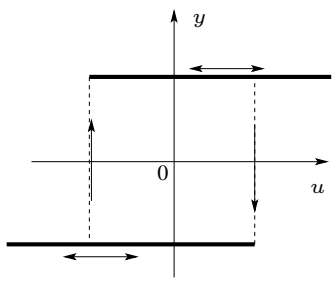

(c)

Fig. 2.3: Hysteresis operators.

used in Lur'e systems and absolute stability framework, extending the celebrated Kalman-Yakubovich-Popov Lemma [139] to such set-valued feedback loops [335, 457, 469]. The double inclusions alluded to at the end of Section 2.2, are another kind of hysteresis models [557].

REMARK 8. The so-called backlash hysteresis operator that is widely used in Systems and Control [334], is a form of static model of mechanical clearance, neglecting dynamical effects. If all dynamical effects (like impacts) are modeled, one ends up with a mechanical system with unilateral constraints, impacts and possibly friction, which can be recast into (4.9) below (see [130, Remark 5.15] for the model of mechanical clearance within the $S O S w P)$.

3. Relationships between various formalisms. Starting from (1.1) we see that $0 \in w+\mathcal{F}(t, H x, J w) \Leftrightarrow w \in\left(I_{m}+\mathcal{F}(t, H x, J \cdot)^{-1}(0)\right.$. Therefore the nonsmooth system (1.1) can be equivalently rewritten as the DI:

$$
\dot{x}-f(t, x) \in\left(I_{m}+\mathcal{F}(t, H x, J \cdot)^{-1}(0) .\right.
$$

Characterizing the operator on the right-hand side is crucial to understand our systems, and their relationships. Some relationships between some nonsmooth formalisms as those presented above, have been analysed in various articles [308, 266, 143, 133, 229, 462]. We review them deeply in the next sections. 
3.1. Maximal monotone DIs, complementarity systems, FOSwP, and DVIs. Let the set $K$ be closed non empty and convex. We notice at once that the DVIs in (2.21) can be equivalently rewritten as:

$$
\left\{\begin{array}{l}
\dot{x}=f(t, x)+G(t, x) w \\
z=\Psi(t, x, w) \\
z \in-\mathcal{N}_{K}(w)
\end{array}\right.
$$

since the inequality in (2.20) is equivalently rewritten as $\Psi(\lambda) \in-\mathcal{N}_{K}(\lambda)$. In case $z=$ $C x+D w+g(t)$, we see that the DVI is equivalent to the DI: $\dot{x} \in f(t, x)-G(t, x)(D+$ $\left.\mathcal{N}_{K}\right)^{-1}(C x+g(t))$. The DVI is thus a particular DI, where the presence of the "input" term $G(t, x)$ prevents in general maximal monotonicity of the operator $x \mapsto$ $G(t, x)\left(D+\mathcal{N}_{K}\right)^{-1}(C x)$ for each $t$. Consequently the results on maximal monotone DIs as (2.16) may not apply, one exception being when passivity-like input-output constraints are imposed [143, Section 4]. Let $K$ be a closed convex cone, then using (B.1) and letting $D=0$ yields $\left(D+\mathcal{N}_{K}\right)^{-1}(C x+g(t))=\mathcal{N}_{\mathcal{S}(t)}(C x)$ with $\mathcal{S}(t)=$ $K^{\star}-g(t)$. In this case one recovers a particular form of FOSwP (and a generalized FOSwP if $\left.G(t, x) \neq I_{n}\right)$. However FOSwP are formulated in the more general context of closed convex sets $\mathcal{S}(t)$.

REMARK 9. The signum multifunction with $\operatorname{sgn}(0)=[-1,1]$ is related to the normal cone $\mathcal{N}_{[-1,1]}(\cdot)$ in the following sense: we have $\mathcal{N}_{[-1,1]}(x)=\partial \psi_{[-1,1]}(x)$, and $\operatorname{sgn}(y)=\partial \psi_{[-1,1]}^{*}(y)$, where $\psi^{*}(\cdot)=\sigma_{[-1,1]}(\cdot)=|\cdot|$ is the support function of $[-1,1]$ (see Section A.2.4). Thus relay systems (2.35) and inclusions in normal cones to hypercubes have set-valued parts which are conjugate one to each other. We may consider the following system: $\dot{\xi}=A^{T} \xi+C^{T} \zeta, \lambda=B^{T} \xi+D \zeta, \zeta \in-\mathcal{N}_{[-1,1]^{m}}(\lambda)$. Then this system and (2.35) (let us take $E_{1}=0, E_{3}=0, E_{4}=0$ for simplicity) are rewritten respectively in an equivalent way as the DIs:

$$
\left\{\begin{array}{l}
\dot{\xi} \in A^{T} \xi+C^{T}\left(D+\partial \psi_{[-1,1]^{m}}^{*}\right)^{-1}\left(-B^{T} \xi\right) \\
\dot{x} \in A x+B\left(D+\partial \psi_{[-1,1]^{m}}\right)^{-1}(-C x)
\end{array}\right.
$$

which we consider as dual one to each other, the second system being a DVI, the first one being similar to (2.14) (see (2.15)). A next step is to consider a control input $u$ in the x-dynamics and a measured output $y$ in the $\xi$-dynamics, and to investigate their Systems and Control properties.

A variety of problems that can be recast as DVIs, are presented in [462]. The set $K$ can take various forms (this is obviously true also for the FOSwP, or maximal monotone DIs with $\mathcal{F}(t, x)=\mathcal{N}_{\mathcal{S}}(x)$ ), for instance $K=[a, b] \times \bar{K} \times S \subset \mathbb{R}^{1+p+m}$, where $a, b \in \mathbb{R}$, $\bar{K} \subseteq \mathbb{R}^{p}$ is closed convex, and $S=\left\{v \in \mathbb{R}^{m} \mid A v+B=0\right\}$ for some constant $A \in \mathbb{R}^{l \times m}$, $B \in \mathbb{R}^{l}$. Then $\mathcal{N}_{K}\left(\left(z_{1}, z_{2}, z_{3}\right)\right)=\left(\begin{array}{c}\mathcal{N}_{[a, b]}\left(z_{1}\right) \\ \mathcal{N}_{\bar{K}}\left(z_{2}\right) \\ \mathcal{N}_{S}\left(z_{3}\right)\end{array}\right), \mathcal{N}_{S}\left(z_{3}\right)=\operatorname{Im}\left(A^{T}\right)$. Thus it is possible to include equality constraints $h(t, x) \in S$ by properly choosing both $K$ and $\Psi(t, x, w)$ (this is, anyway, the case for all inclusions into normal cones). Let us now assume that $K$ is finitely represented, i.e., $K=\left\{z \in \mathbb{R}^{m} \mid h_{i}(z) \geq 0,1 \leq i \leq p\right\}$ for some continuously differentiable functions $h_{i}: \mathbb{R}^{m} \rightarrow \mathbb{R}$. If the functions $h_{i}(\cdot)$ satisfy a constraint qualification like the MFCQ (see Section A.1.3), then the normal cone is equal to its linearization cone, and we can rewrite equivalently the DVI as an NLCS (2.24).

Consider now the DI in (2.17). If $\mathcal{M}(\cdot)=\mathcal{N}_{K}^{-1}(\cdot)=\sigma_{K}(\cdot)$, then (2.17) can be recast into (3.1), hence into $(2.21)$. If $\mathcal{M}(\cdot)=\partial \varphi(\cdot)$ for some proper convex LSC $\varphi(\cdot)$ has 
a PWL graph, then from the algorithm in [551] one can rewrite (2.17) as an MLCS (2.25).

EXAMPLE 3. Let us consider a simple mechanical system with Coulomb friction: $m \ddot{q}+c \dot{q}+k q \in-\mu \partial|\dot{q}+f(t)|=\mu \operatorname{sgn}(-\dot{q}-f(t)), \mu>0, k \geq 0, c \geq 0, f(\cdot)$ continuous. This belongs to the class of relay systems in (2.35), so that the equivalent rewriting (3.12) below, applies. Thus using (B.1), the dynamics is equivalently rewritten as: $\dot{x}=$ $A x+\left(\begin{array}{c}0 \\ \frac{\mu}{m}\end{array}\right)+\left(\begin{array}{cc}0 & 0 \\ -\frac{2 \mu}{m} & 0\end{array}\right) w, w \in-\mathcal{N}_{\mathbb{R}_{+}^{2}}(h(x, w)), h(x, w)=\left(\begin{array}{c}-x_{2}-f(t) \\ 1\end{array}\right)+$ $\left(\begin{array}{cc}0 & 1 \\ -1 & 0\end{array}\right) w, x=\left(x_{1}, x_{2}\right)^{T}, x_{1}=q, x_{2}=\dot{q}$. This fits with (3.1), with $h(x, w)=$ $\Psi(t, x, w)$ and using (B.1), noting that $\left(\mathbb{R}_{+}^{2}\right)^{\star}=\mathbb{R}_{+}^{2}$. On the other hand, the dynamics can also be written as: $m \ddot{q}+c \dot{q}+k q=\lambda,\langle\lambda, v-\dot{q}-f(t)\rangle+\varphi(v)-\varphi(\dot{q}+f(t)) \geq 0$ for all $v \in \mathbb{R}, \varphi(\cdot)=\mu|\cdot|$, and it can also be written in a complementarity form, see Section 3.10.

This example shows that the same system, can be written either as a DVI, or as a DVI of the second kind, or as an LCS. Same holds for slightly more complex systems (controlled with a PID input) as in [108]. However in general this manipulation is not doable. The generalization of DVIs towards DVIs of the second kind (called differential mixed VI in [389]) has been considered in [283] (see [14, Section 3.2] [278, Section 5.2]), in a more general setting in [389], in an infinite-dimensional context in $[395,394]$. As alluded to in Remark 2, inspection of [462, Theorem 6.1], shows that it is mainly the cases with relative degree zero systems, which are considered in [462] for well-posedness issues (with AC solutions). Hence SOSwP and HOSwP are excluded, as they would require to define measure or distribution DVIs.

3.2. FOSwP, SOSwP, EVIs and QVIs. Evolution (or dynamical) VIs and sweeping processes are known to be close formalisms [512], mainly due to the fact that the normal cone to a convex set, can be expressed in a variational way, see e.g. (A.2). This was actually one of the original manipulations made to express elasto-plastic problems, as a FOSwP. The next equivalences follow from the definition of a normal cone to a closed convex set. To start with, it is clear that the FOSwP in (2.2) can be equivalently written as:

$$
\left\{\begin{array}{l}
x(t) \in \mathcal{S}(t), \text { for all } t \geq 0 \\
\left\langle\dot{x}-f(t, x), x^{\prime}-x\right\rangle \geq 0 \text { for all } x^{\prime} \in \mathcal{S}(t)
\end{array}\right.
$$

which is an evolution variational inequality (EVI). Let $\mathcal{S}(t)$ be closed convex for each $t$. Using the conjugate function $\partial \psi_{\mathcal{S}(t)}^{*}=\partial \sigma_{\mathcal{S}(t)}$, the FOSwP is recast into a DI as $(2.17): \dot{x} \in f(t, x)+\left(0+\partial \psi_{\mathcal{S}(t)}^{*}\right)^{-1}(x)$. The FOSwP with state-dependent set $\mathcal{S}(t, x)$ yields evolution QVIs (see (A.7) in Section A.2.2). The SOSwP in (2.6) can in turn be written as:

$$
\left\{\begin{array}{l}
q(t) \in \Phi, v(t) \in V(q(t)), \text { for all } t \geq 0 \\
\left\langle d v-f(t, q, v), v^{\prime}-v_{e}\right\rangle \geq 0 \text { for all } v^{\prime} \in V(q),
\end{array}\right.
$$

which is an evolution measure QVI. The $\mathrm{SOSwP}$ of the form: $\ddot{u} \in-B \mathcal{N}_{\mathcal{S}(t, u, \dot{u})}(\dot{u})+$ $F(t, u, \dot{u})+A u$ (see Section 2.1.2) also yields a kind of QVI, where the set $K(x) \triangleq$ $\mathcal{S}(t, u, \dot{u})$ in (A.7) has quite different properties than the tangent cone $V(q)$ (the main discrepancy being the compactness). See Section 3.7.

REMARK 10. What we name EVI is not to be confused with EVIs as in [213, 214, 195], which are VIs that vary with time, whose solutions thus represent time-varying equilibria (trajectories) of our EVIs which are dynamical systems. 
3.3. FOSwP and compact DIs. Under some basic assumptions, it is possible to show that the FOSwP is equivalent (in the sense that both dynamical systems have the same set of solutions) to a DI of the form: $\dot{x} \in-|\dot{v}(t)| \partial_{C} d_{\mathcal{S}(t)}(x), x(t) \in \mathcal{S}(t)$ for all $t \geq 0$, where $v(\cdot)$ is $\mathrm{AC}$ and is such that $\left|d\left(x, \mathcal{S}\left(t_{1}\right)\right)-d\left(x, \mathcal{S}\left(t_{2}\right)\right)\right| \leq\left|v\left(t_{1}\right)-v\left(t_{2}\right)\right|$ for all $x \in \mathbb{R}^{n}$ and all $t_{1}, t_{2}, \partial_{C}$ is the Clarke subdifferential (see Section A.2.1). This is known as the reduction method of FOSwP, and it is proved in [529] when $\mathcal{S}(t)$ is convex, extended in [530] for Fréchet normally regular ${ }^{5}$ and prox-regular sets, using techniques similar to the convex case, see also [113, Theorems 2.4, 2.5] $]^{6}$, and in [295] for $\alpha$-far and AC sets. The set-valued right-hand side of the new DI is compact, and is contained in the normal cone to $\mathcal{S}(t)$ (thus, it is a "smaller" inclusion). This can be used to prove existence of solutions to the FOSwP. Related results are presented in [122] where FOSwP are approximated with suitably controlled ODEs.

3.4. Complementarity systems, FOSwP, relay systems and maximal monotone DIs. To begin with, let us consider the FOSwP in (2.2), and assume that for each $t, \mathcal{S}(t)$ is a closed convex cone. Then using (B.1), we can rewrite (2.2) as

$$
\left\{\begin{array}{l}
\dot{x}-f(t, x)=\eta \\
\mathcal{S}(t) \ni z=x(t) \perp \eta(t) \in \mathcal{S}^{\star}(t)
\end{array}\right.
$$

which is a cone complementarity system, with relative degree $r=1$ between the "output" $z$ and the "input" $\eta$ (directly from the relative degree definition in Appendix C).

Notice that using (B.1) with $K=K^{\star}=\mathbb{R}_{+}^{m}$ and choosing $\mathcal{M}(\cdot)=\partial \psi_{\mathbb{R}_{+}^{m}}(\cdot)$, it follows that (2.17) is an LCS as in (2.22). Apparently the equivalence between maximal monotone DIs as in (2.16) with $f(t, x)=f(x)=A x$ linear, $\mathcal{F}(t, x)=\mathcal{F}(x)$ time invariant, and LCS, has been first shown in [126], under a positive real condition [139] on the transfer function $D+C(s I-A)^{-1} B, s \in \mathbb{C}$. A key state space transformation was introduced in [126], assuming the existence of $P=P^{T} \succ 0$ such that $P B=C^{T}$, then defining $z=R x$ with $R=R^{T} \succ 0, R^{2}=P$ (which is a consequence of (D.1) when $D+D^{T}=0$ ). It allows one to use the chain rule from Convex Analysis when $\mathcal{F}(x)=B \partial \varphi(x)$ for a convex proper LSC $\varphi(\cdot)$.

$\rightsquigarrow$ This transformation has been used afterwards in [9, 11, 12, 20, 28, 36, 31, 39, 33, 134, 135, 136, 138, 143, 159, 280, 375, 386, 522, 524], extended in [523, 159] and in [143, Section 4] for the nonlinear case.

Let the quadruple $(A, B, C, D)$ of the LCS in (2.22) be passive (see Section D). Let $D$ be full rank $\left(\Rightarrow D \succ 0\right.$, see (D.1)), then the vector relative degree $\bar{r}=(0,0, \ldots, 0)^{T} \in$ $\mathbb{R}^{m}$, and as pointed out in Section 2.4.1, in this case the LCS is an ODE with Lipschitz vector field, applying (B.1) and (A.6) (the complementarity conditions partition the state space into polyhedral cells whose boundaries depend on $u$ through $G$ ). Let $D=0$, then $P B=C^{T} \Rightarrow C B=B^{T} P B \succcurlyeq 0$. It follows that $\dot{z}=C(A x+B \lambda+$ $\left.E_{1} u+E_{2}\right)+E_{3} u+E_{4}=C B \lambda+C A x+C E_{1} u+C E_{2}$. Thus $C B$ is the input/ouput decoupling matrix. If the pair $(C, A)$ is observable $(\Rightarrow P \succ 0[139,155])$ and $B$ has full column rank, then $C B \succ 0$ and $\bar{r}=(1,1, \ldots, 1)^{T} \in \mathbb{R}^{m}$. A characterization of passive systems via their transfer matrix $\mathcal{H}(s)=D+C(s I-A)^{-1} B$ index $^{7}$ is also possible, showing that their total index is one [306, Theorem 3.14]. Let us assume

\footnotetext{
${ }^{5}$ i.e., $\hat{\mathcal{N}}_{\mathcal{S}(t)}(x)=\mathcal{N}_{\mathcal{S}(t)}^{C}(x)$ for all $x \in \mathcal{S}(t)$ and all $t$, see Section A.1.2 for normal cones definitions.

${ }^{6}$ Both results in [530] and [113] were in fact submitted at the same date.

${ }^{7}$ The index and the relative degree are intimately related, though no formal proof of their exact relationships seems to be available in the Systems and Control literature.
} 
therefore that $D=0$ and $P B=C^{T}, P=P^{T} \succ 0$, then, as shown in [143] the LCS (2.22) can be rewritten equivalently as the FOSwP:

$$
-\dot{\xi}+R A R^{-1} \xi+R E_{1} u+R E_{2} \in \mathcal{N}_{\Phi(u(t))}(\xi)
$$

where $R^{2}=P, \xi=R x, \Phi(u(t)) \triangleq\{R x \mid x \in K(t)\}, K(t) \triangleq\left\{x \in \mathbb{R}^{n} \mid C x+E_{3} u(t)+\right.$ $\left.E_{4} \geq 0\right\}$, both convex polyhedral time-varying sets. The transformation mainly relies on the use of the chain rule in Proposition A.3 and on (B.1). The regularity of $\Phi(\cdot)$ depends on the regularity of $u(\cdot)$. As shown in [143, Proposition 3.2], under the constraint qualification $\operatorname{Im}(C)-\mathbb{R}_{+}^{m}=\mathbb{R}^{m}$, one has $u \in$ locally $\mathrm{AC} \Rightarrow \Phi \in$ locally $\mathrm{AC}$, $u(\cdot)$ is right-continuous $\Rightarrow \Phi(\cdot)$ is right-continuous, and $u \in R C L B V \Rightarrow \Phi \in R C L B V$. The set of discontinuity times of $\xi(\cdot)$, is included in that of $u(\cdot)$ (a fact also proved in [151] in the context of passive LCS). It is then easy to apply (2.12) and Lemma 2.3 to characterize state jumps.

Remark 11. Passive LCS (2.22) with $D=0$ satisfy $P B=C^{T}$, see (D.1). Hence they can be recast into (3.6). This, together with the fact that passive LCS with $D \succ 0$ are ODEs with Lipschitz right-hand side, implies that LCS (2.22) with rightcontinuous $u(\cdot)$ (or with $G=0$ ) can have state jumps only initially, a fact noticed in [151]. This follows also using (3.6) and the definition of $K(t)$. Moreover the set of state jumps times is included in the set of discontinuities in $u(t)$ (or in $K(t)$ ). We infer that passive LCS with right-continuous $u(\cdot)$, have (except initially) the property that a bounded multiplier $\lambda(t)$ is sufficient to integrate them and keep the state $x$ inside the admissible domain (when $D=0$ ).

In a more general setting, one may assume that $\operatorname{ker}\left(D+D^{T}\right) \subset \operatorname{ker}\left(P B-C^{T}\right)$ for some matrix $P=P^{T} \succcurlyeq 0$. This is a necessary condition for passivity $[155,159]$. The above material can then be extended to non zero feedthrough matrices $D$ [524], giving rise to some extension of the degenerate FOSwP (see Section 2.1.1). However the normal cone argument still depends on $\eta$, hence the transformed system is not a FOSwP: it is a DI as in (2.16), with a matrix $B$ premultiplying $\Phi(t, x)$, and $v=C x+D \eta$, in (2.14b) [524]: the assumption $\operatorname{ker}\left(D+D^{T}\right) \subseteq \operatorname{ker}\left(P B-C^{T}\right)$ is key to recover a maximal monotone DI, see also Section 5.3.1. In [143, Section 3.3] a special block-diagonal structure for $D \succcurlyeq 0$ was assumed, and the LCS could be recast after splitting the complementarity variables, into a cone LCS which lends itself to a FOSwP formulation (a similar idea was used in [135]).

Another way to prove the link between FOSwP and complementarity systems (not necessarily LCS) is as follows, as shown by Moreau [443]. Consider (2.2). Assume that $\mathcal{S}(t) \triangleq\left\{x \in \mathbb{R}^{n} \mid h(x, t) \geq 0\right\}$ for $m$ differentiable functions $h_{i}: \mathbb{R}^{n} \times \mathbb{R}_{+} \rightarrow \mathbb{R}$ and that the MFCQ holds for $\mathcal{S}(t)$ for each $t$ (see Section A.1.3). Thus $\mathcal{N}_{\mathcal{S}(t)}(x)=$ $\left\{w \in \mathbb{R}^{n} \mid w=-\sum_{i \in \mathcal{I}(x, t)} \lambda_{i} \nabla h_{i}(x, t), \lambda_{i} \geq 0\right\}$, with $\mathcal{I}(x, t)=\left\{i \mid h_{i}(x, t)=0\right\}$. We thus obtain $w=-\nabla h(t, x) \lambda$ with $\lambda_{j}=0$ for all $j \notin \mathcal{I}(x, t)$, so (2.2b) is rewritten equivalently as the NLCS: $\dot{x}-f(t, x)=\nabla h(x, t) \lambda, 0 \leq \lambda \perp h(x, t) \geq 0$. Letting $z_{1}=h(x, t)$, we obtain $\dot{z}_{1}=\nabla h(x, t)^{T} \nabla h(x, t) \lambda+\nabla h(x, t)^{T} f(t, x)+\frac{\partial h}{\partial t}(x, t)$. If the constraints are functionally independent in a neighborhood of $x_{0}$, the decoupling matrix $\nabla h(x, t)^{T} \nabla h(x, t)$ is positive definite, showing that in essence the FOSwP has a uniform vector relative degree $\bar{r}=(1, \ldots, 1)^{T} \in \mathbb{R}^{m}$ at $x_{0}$. This allows us to state that the optimal control problem formulated for DIs as (2.16) and with $\mathcal{F}(x)=\mathcal{N}_{\mathcal{S}}(x)$ and $\mathcal{S}$ finitely represented [206, 205, 217], can be interpreted as optimal control of a class of complementarity systems. Whether or not this covers [555] is an open question.

EXAMPLE 4. Consider the scalar FOSwP: $\dot{x} \in-\mathcal{N}_{[t, t+1]}(x), x\left(t_{0}\right) \in[0,1]$. This is equivalently rewritten as the LCS: $\dot{x}=\lambda_{1}-\lambda_{2}, 0 \leq \lambda_{1} \perp x-t \geq 0,0 \leq \lambda_{2} \perp t+1-x \geq$ 
0 . Here $\mathcal{I}(x, t)=\{1\}$ or $\{2\}$ or $\emptyset$, but never $\{1,2\}$. Let $x\left(t_{0}\right)=\frac{1}{2}$, then $x(t)=x(0)$ for all $t \in\left[t_{0}, t_{1}\right]$ with $t_{1}=\frac{1}{2}$. At $t_{1}$ one has $0 \leq \lambda_{1}(t) \perp \lambda_{1}(t)-1 \geq 0$, and employing the material in Section 2.4.1, one finds that $0 \leq \lambda_{1}(t) \perp \dot{x}(t)-1=\lambda_{1}(t)-1 \geq 0$, hence $\lambda_{1}(t)=1$ for $t \geq t_{1}$. Thus $x(t)=t$ for all $t \geq \frac{1}{2}$, and the other constraint is never attained.

REMARK 12 (Relay Systems). It is noteworthy that we can apply the same transformation as above to the ideal relay system in (2.35), assuming that $D=0$ and $P B=C^{T}, P=P^{T} \succ 0$, and we obtain the differential inclusion $\dot{\zeta}-R A R^{-1} \zeta-$ $R E_{1} u \in-R^{-1} C^{T} \partial f\left(C R^{-1} \zeta\right)=-\partial g(\zeta)$, where $f(z)=\left|z_{1}\right|+\ldots\left|z_{n}\right|, \zeta=R x$, and $g(\cdot)$ is convex proper $L S C$ (under a basic constraint qualification such that the chain rule in Proposition A.3 holds, we thus conclude about the uniqueness of AC solutions, hence extending [480, Theorem 2]). Therefore $\partial g(\cdot)$ is maximal monotone and the relay system fits with (2.16) with time-invariant $\mathcal{F}(x)$. Finally notice that (2.35)(b) with $z=C x+D \lambda+E_{3} u+E_{4}$ (we add a control inside the relay multifunction), is equivalent to $C x+E_{3} u+E_{4}+D \lambda \in \mathcal{N}_{[-1,1]^{m}}(-\lambda) \Leftrightarrow \lambda \in-\left(D+\partial \psi_{[-1,1]^{m}}\right)^{-1}\left(C x+E_{3} u+E_{4}\right)$ which is another equivalent expression of the fundamental operator for this Lur'e system. Applying [135, Propositions 1, 2, 3, Corollary 1] we can deduce conditions such that $\lambda$ is a Lipschitz continuous function of $C x+E_{3} u+E_{4}$, and the relay system is an ODE with Lipchitz right-hand side. For instance, $D=D^{T} \succ 0$ implies that $\lambda=\operatorname{proj}_{D}\left[[-1,1]^{m} ;-D^{-1} C x\right]$, where (A.6) and the support function definition are used (see Section A.2.4): the relay system (2.35) is an ODE with Lipschitz continuous right-hand side in this case. It becomes clear that relative degree zero and one relay systems and LCS, belong to the same family of dynamical systems, a fact which may also be noticed by rewriting the signum multifunction in a complementarity formalism [149, §7.5.1], see Section 3.10.

A general link between FOSwP with time-invariant prox-regular $\mathcal{S}$ (hence locally hypomonotone normal cone mapping, see Definition A.2) and DIs with maximal monotone right-hand side as (2.16), is made in [23, Corollary 4.1] (the maximal monotone operator is not constructed explicitly, however).

Let us end this section with time-varying systems. Consider first the case where $C=C(u)$ in (2.22b), with $E_{3}=0$ and $D=0$. We get $0 \leq \lambda \perp z=C(u) x+$ $E_{4} \geq 0$, equivalently using (B.1): $\lambda \in-\partial \psi_{\mathbb{R}_{+}^{m}}\left(C(u) x+E_{4}\right)$. If we assume that $B=B(u)=C(u)^{T}$, we obtain using the chain rule (apply Proposition A.3 with the suitable assumptions) that: $\dot{x}-A x \in-\mathcal{N}_{\Phi(u(t))}(x)$ with $\Phi(u(t)) \triangleq\left\{x \in \mathbb{R}^{n} \mid C(u(t)) x+\right.$ $\left.E_{4} \geq 0\right\}$. The set $\Phi(u(t))$ in (3.6) is a moving fixed-shape polyhedral set, while this one is a "fixed" moving-shape polyhedral set, both being convex for each $u(t)$ (if non empty). These two FOSwPs will certainly possess quite different controllability properties. Let us now consider a time-varying LCS with $(A(t), B(t), C(t), 0)$ and matrix functions $E_{1}(t), E_{3}(t)$ in $(2.22)$, with sufficient smoothness. Assume that there exists $P(t)=P^{T}(t) \succ 0$ such that $P(t) B(t)=C^{T}(t)$ for all $t$ (this is satisfied by passive systems with $D(t)=0$ and observable pair $(A(t), C(t))$ for all $t$ [52, Lemma 3] [139, Lemma 3.66]). Let $z=R(t) x$, with $R(t)=R^{T}(t) \succ 0$ and $R^{2}(t)=P(t)$ for all $t$. Applying the chain rule (see Section A.2.3, and doing suitable assumptions not given here), one obtains the FOSwP: $\dot{z}-\left(\dot{R}(t) R(t)+R(t) A(t) R^{-1}(t)\right) z-R(t) E_{1}(t) u(t) \in$ $-\partial \psi_{\mathcal{S}(t, u(t))}(z)$, where $\mathcal{S}(t, u(t))=\left\{w \in \mathbb{R}^{n} \mid C(t) R^{-1}(t) w+E_{3}(t) u(t) \geq 0\right\}$. One has to analyse further the conditions such that the set $\mathcal{S}(t, u(t))$ varies in a suitable way (AC, BV, or else), so that existence of solutions may be proved. Finally it is clear from the above that the analysis of time-delay FOSwP may be used in the framework 
of complementarity systems.

3.5. ZOSwP, complementarity systems, projected systems. Consider the ZOSwP as defined at the end of Section 2.1.1 [27]: $A_{1} \dot{x}+A_{0} x \in-\mathcal{N}_{\mathcal{S}(t)}(\dot{x}), A_{1} \succcurlyeq$ 0 . Let $\operatorname{rank}\left(A_{1}\right)=p \leq n$, hence one obtains a kind of differential-algebraic DI, different from (2.18) in two aspects: time-variation and the presence of $\dot{x}$ in the normal cone. Assume that $S(t)$ is a cone for each $t$. Doing as in (3.5), we can write the complementarity condition: $\mathcal{S}(t) \ni \dot{x} \perp z=A_{1} \dot{x}+A_{0} x \in \mathcal{S}^{\star}(t)$. The relative degree $r=0$ between this output $z$ and its complementary variable $\dot{x}$ if $p=n$. Otherwise the relative degree interpretation requires further analysis (in a sense, the loss of relative degree zero is compensated for by the surjectivity of the normal cone when $\mathcal{S}(t)$ is bounded, in [27]). The case when $\mathcal{S}(t)$ is just closed convex, but finitely represented, is treated now, showing that this ZOSwP yields a new kind of LCS, that may be called an implicit complementarity system. Assume that $h(x, t)=B(t) x+g(t)$ for some regular enough $B: \mathbb{R}_{+} \rightarrow \mathbb{R}^{m \times n}$ and $g(\cdot)$, and that the MFCQ holds for each $t$ for the family $\left\{h_{i}(x, t)\right\}_{1 \leq i \leq m}$. Then $\mathcal{N}_{\mathcal{S}(t)}(\dot{x})=\left\{w \in \mathbb{R}^{n} \mid w=-B(t)^{T} \lambda, 0 \leq \lambda \perp B(t) \dot{x}+g(t) \geq 0\right\}$, see Section A.1.3. Thus this ZOSwP is rewritten as: $A_{1} \dot{x}+A_{0} x=B(t)^{T} \lambda, 0 \leq \lambda \perp$ $z=B(t) \dot{x}+g(t) \geq 0$, which is a kind of implicit LCS as long as $\operatorname{rank}\left(A_{1}\right)=p<n$ (if $p=n$ one can easily recover a time-varying LCS with $\left.D(t)=B(t) A_{1}^{-1} B(t)^{T}\right)$. Such a ZOSwP was introduced in [39], where a circuit example is also given.

Let us now focus on the ZOSwP: $\dot{x} \in-\mathcal{N}_{\mathcal{S}(t)}(A \dot{x}+B x)$ [23]. Proceeding as above and in (3.5), this is equivalent to: $\dot{x}=\lambda, \mathcal{S}(t) \ni z=A \dot{x}+B x \perp \lambda \in \mathcal{S}^{\star}(t)$ when $S(t)$ is a cone for each $t$. The relative degree is $r=0$ when $A$ has full rank. The following holds (which is similar in spirit to Proposition 3.2 appearing later).

Proposition 3.1. Let $\mathcal{S}(t)$ be closed convex and non empty for each $t$. Assume that $A=A^{T} \succ 0$, and let $v \in \mathbb{R}^{n}, x \in \mathbb{R}^{n}$ be two vectors. Then the following holds: (a) $v \in-\mathcal{N}_{\mathcal{S}(t)}(A v+B x) \Leftrightarrow(b) v \in-\left(A+\partial \sigma_{\mathcal{S}(t)}\right)^{-1}(B x) \Leftrightarrow$ (c) $v \in\left(A+\mathcal{N}_{\tilde{\mathcal{S}}(t)}\right)^{-1}(0) \Leftrightarrow(d) v \in-A^{-1} B x+A^{-1} \operatorname{proj}_{A}[\mathcal{S}(t) ; B x] \Leftrightarrow(e) v \in$ $-\left(\partial\left(f+\sigma_{\mathcal{S}(t)}\right)\right)^{-1}(B x)$, where $\tilde{\mathcal{S}}(t) \triangleq\left\{w \in \mathbb{R}^{n} \mid A w \in \mathcal{S}(t) \backslash\{B x\}\right\}, f(v)=\frac{1}{2} v^{T} A v$, $\sigma_{\mathcal{S}(t)}(\cdot)$ is the support function of $\mathcal{S}(t)$ (see Section A.2.4).

Proof. Using the material in Section A.2.4, the first equivalence follows immediately. Multiplying both sides of $(a)$ by $A$ and using the symmetry, one obtains $A v \in-A \partial \psi_{\overline{\mathcal{S}}(t)}(A v)$, with $\overline{\mathcal{S}}(t)=\left\{z \in \mathbb{R}^{n} \mid A z+B x \in \mathcal{S}(t)\right\}$. Using the chain rule in Proposition A.3, one gets $A v \in-\partial \psi_{\tilde{\mathcal{S}}(t)}(v)$, which proves $(a) \Leftrightarrow(c)$. Notice that we can rewrite $(a)$ as $A v+B x-B x \in-A \mathcal{N}_{\mathcal{S}(t)}(A v+B x)$, then using (A.6), (d) follows equivalently. (e) is just a rewriting of $(b)$ using [97, Corollary 16.38]. $\square$

Once the well-posedness has been shown, then all the above inclusions define equivalent DIs, in a similar way to Theorem 3.3. It is interesting to compare the formalisms in Proposition 3.1, with PDS in Section 2.5, and the formalisms in (3.8). We do not discuss here the advantages/drawbacks of these formalisms. One guesses that $(d)$ may be more amenable for numerical simulation, and also for well-posedness [341, Theorem 4.2]. If one assumes that $\mathcal{S}(t)$ is finitely represented, then the link with LCS can be done and the projection can be calculated.

3.6. Degenerate FOSwP, complementarity systems. Let $\mathcal{S}(t)$ be closed convex for each $t$, and be finitely represented, that is $\mathcal{S}(t)=\left\{x \in \mathbb{R}^{n} \mid h(t, x) \geq 0\right\}$, $h: \mathbb{R} \times \mathbb{R}^{n} \rightarrow \mathbb{R}^{m}$. Let us consider a degenerate FOSwP [369, 371, 522] of the form $\dot{x}-f(t, x) \in-\mathcal{N}_{\mathcal{S}(t)}(H x)$, with $H=H^{T} \succ 0$ (hence the mapping $x \mapsto H x$ is maximal strongly monotone). Performing the variable change $y=H x$ we obtain $\dot{y}-H f\left(t, H^{-1} y\right) \in-H \mathcal{N}_{\mathcal{S}(t)}(y)$. It is not possible here to redo the same transfor- 
mation as we did in Section 3.4 to get (3.6), because the normal cone argument is $y$, not $H y$. Nevertheless, assume that the Mangasarian-Fromovitz constraint qualification (MFCQ) is satisfied for the differentiable mappings $\bar{h}_{i}(t, y) \triangleq h_{i}\left(t, H^{-1} y\right)$, then $\mathcal{N}_{\mathcal{S}(t)}(y)$ is equal to its linearization cone (see Section A.1.3), and we have $\mathcal{N}_{\mathcal{S}(t)}(y)=\left\{z \in \mathbb{R}^{n} \mid z=-\nabla \bar{h}(t, y) \lambda, 0 \leq \lambda \perp \bar{h}(t, y) \geq 0\right\}$. The degenerate FOSwP is thus equivalent to the NLCS:

$$
\left\{\begin{array}{l}
\dot{y}-H f\left(t, H^{-1} y\right)=-H \nabla \bar{h}(t, y) \lambda \\
0 \leq \lambda \perp \bar{h}(t, y) \geq 0
\end{array}\right.
$$

See also [522, Section 3.1.2] for related results about LCS.

3.7. SOSwP and complementarity systems. We will see in details the relationships between the SOSwP in (2.6), and Lagrangian complementarity systems, in Section 4.2.1. Let us deal here with SOSwP: $\ddot{u}-f(t, u, \dot{u}) \in-\mathcal{N}_{\mathcal{S}(u)}(\dot{u})$, where $\mathcal{S}(u)$ is supposed to possess some properties (among these, compactness for all $u$ [43]). Let us assume that $\mathcal{S}(u)=\left\{v \in \mathbb{R}^{n} \mid C(u) v+D(u) \geq 0\right\}$, for some $C: \mathbb{R}^{n} \rightarrow \mathbb{R}^{m} \times \mathbb{R}^{n}$, $D: \mathbb{R}^{n} \rightarrow \mathbb{R}^{m}$. The set $\mathcal{S}(u)$ being polyhedral (in the space of velocities $\dot{u}$ ), one has $\mathcal{N}_{\mathcal{S}(u)}(\dot{u})=\left\{z \in \mathbb{R}^{n} \mid z=C^{T}(u) \lambda, 0 \leq \lambda \perp C(u) \dot{u}+D(u) \geq 0\right\}$, see Section A.1.3. Thus we obtain an NLCS (2.24): $\ddot{u}-f(t, u, \dot{u})=C^{T}(u) \lambda, 0 \leq \lambda \perp z=C(u) \dot{u}+D(u) \geq 0$. Differentiating $z$ once gives $\dot{z}=C(u) C^{T}(u) \lambda+\frac{d}{d t}(C(u))+\ldots$, showing that basically the relative degree is equal to one (at least, the decoupling matrix $C(u) C^{T}(u) \succcurlyeq 0$ and $\neq 0$ ). In case of the $\operatorname{SOSwP}$ in $(2.6)$, the relative degree is always $>1$, because the "output" (the complementarity variable $z$ ) is not the same: it is a constraint on $u$, not on $\dot{u}$, because the tangent cone $V(q)$ (which plays the role of $\mathcal{S}(u)$ ) has a switching structure, that is seen through the presence of an active constraints index set, see the definition of $\mathcal{T}_{K}^{h}(x)$ in Section A.1.3. More details are in Section 4.2.1. This little analysis shows intuitively why both kinds of SOSwP are not the same.

3.8. Projected dynamical systems, DIs and complementarity systems. Consider the PDS in (2.31). Let us introduce the differential inclusions, VIs and QVIs and complementarity system, where $K \subseteq \mathbb{R}^{n}$ is closed convex non empty:

(a) $-\dot{x} \in f(x)+g(t)+\mathcal{N}_{\mathcal{T}_{K}(x)}(\dot{x})$,

(b) $-\dot{x} \in f(x)+g(t)+\mathcal{N}_{K}(x)$,

(c) $(x, \dot{x}) \in K \times \mathcal{T}_{K}(x)$ and $\langle\dot{x}+f(x)+g(t), y-\dot{x}\rangle \geq 0$ for all $y \in \mathcal{T}_{K}(x)$,

(d) Let $K=\left\{x \in \mathbb{R}^{n} \mid h_{i}(x) \leq 0,1 \leq i \leq m\right\}:-\dot{x}=f(x)+g(t)+\nabla h(x) \lambda$, $0 \leq z=-h(x) \perp \lambda \geq 0$

(e) $x(t) \in K$ and $\langle\dot{x}+f(x)+g(t), y-x(t)\rangle \geq 0$, for all $y \in K$,

where the functions $h_{i}(\cdot)$ are supposed to be continuously differentiable. The dynamics in (3.8) (a) has an implicit form, like the ZOSwP in [23] (see Proposition 3.1). This is however not a complementarity system with relative degree $r=0$, since it is equivalent under some constraint qualification (see Theorem 3.3), to (3.8) (d). If the functions $h_{i}(\cdot)$ are linearly independent in a neighborhood of some $x_{0}$, then the matrix $\nabla h^{T}\left(x_{0}\right) \nabla h\left(x_{0}\right)$ is invertible so that $r=1$ locally. This shows that projecting on $\mathcal{T}_{K}(x)$, modifies significantly the dynamics compared with the formalisms of Proposition 3.1. The following results hold.

Proposition 3.2. [133, Corollary 2] Given two vectors $x=x(t) \in K$ and $\dot{x}=\dot{x}(t) \in \mathbb{R}^{n}$, the following three statements are equivalent:

(i) The PDS in (2.31) holds;

(ii) The implicit differential inclusion in (3.8) (a) holds; 
(iii) The differential inclusion in (3.8) (b) holds, together with the following two equivalent properties:

$(\text { iii })_{1}-\dot{x}(t)=f(x(t))+g(t)+\operatorname{proj}\left[\mathcal{N}_{K}(x(t)) ;-f(x(t))-g(t)\right]$.

(iii) $)_{2}$ the vector $-\dot{x}(t)$ is of minimum norm in $f(x(t))+g(t)+\mathcal{N}_{K}(x(t))$.

The proof uses Moreau's two cones Lemma, as well as (A.6). From (iii) $)_{2}$, if $x(t) \in$ $\operatorname{int}(K)$, then $-\dot{x}(t)=f(x(t))+g(t)$, while if $x(t) \in \mathrm{bd}(K)$, there exists a selection $\lambda(t) \in \mathcal{N}_{K}(x(t))$ such that $\lambda(t)+f(x(t))+g(t)$ has minimum norm: this is what (iii) 1 says, that $\lambda(t)=\operatorname{proj}\left[\mathcal{N}_{K}(x(t)) ;-f(x(t))-g(t)\right]$. It clearly appears from Proposition 3.2 that the equivalent form of (2.31) is the implicit DI in (3.8) (a). The DI in (3.8) (b) is equivalent to the PDS if and only if it has the so-called slow solution (with minimal $\dot{x}$ ). Proposition 3.2 uses only geometrical arguments. We note that the QVI (3.8) (c) is just a rewriting of the normal cone in (3.8) (a), while the VI (3.8) (e) is a rewriting of the normal cone in (3.8)(b) in terms of subgradients from Convex Analysis. The NLCS in (3.8) (d) is the rewriting of the normal cone in a complementarity framework, and is equivalent to (3.8) (b) under a constraint qualification guaranteeing that $\mathcal{N}_{K}(\cdot)=\mathcal{N}_{K}^{h}(\cdot)$ (see Section A.1.3). Results similar to Proposition 3.2 are presented in [303, Lemma 4.5, Corollary 6.8], when $K$ is just tangentially regular (see Section A.1.1), and the projection is made in a non Euclidean metric $M(x)$. Let us now state a result that involves solutions of the evolution problems.

Theorem 3.3. [133, Theorem 1] Assume that $g \in \mathcal{L}^{1}\left(\mathbb{R}_{+}, \mathbb{R}^{n}, d t\right), f(\cdot)$ is continuous over $\mathbb{R}^{n}$ and hypomonotone. Then for any initial condition $x(0)=x_{0} \in K$, the $D I$ (3.8) (b) has a unique solution $x(t)$ on $\mathbb{R}_{+}$, which is slow: $\dot{x}(t)$ is of minimal norm in the set $f(x(t))+g(t)+\mathcal{N}_{K}(x(t))$. Under these conditions, the systems in (2.31), (3.8) (a) and (3.8) (b) have the same unique solution, which satisfies $\dot{x}(t) \in \mathcal{T}_{K}(x(t))$. Under the MFCQ, the same holds for the NLCS (3.8) (d).

Hypomonotonicity is defined in Section A.2.1, the MFCQ is defined in Section A.1.2. The MFCQ is sufficient for proving the equivalence with the NLCS. Indeed the NLCS is equivalent to (3.8) (b) where $\mathcal{N}_{K}(x)$ is replaced by its linearization cone $\mathcal{N}_{K}^{h}(x)$, which in turn is equal to $\mathcal{N}_{K}(x)$ under the MFCQ, see Section A.1.2. Slow (or lazy) solutions play a significant role in our systems, a well-known fact for DIs as in (2.16) with maximal monotone $\mathcal{F}(x)$ [62, Chapter 3, Theorem 1]. See also [242] when $K$ is closed convex, and the close link with viability [62]. Notice that the element of minimum norm in a non empty convex set $K(t, x)$ is equal to $\operatorname{proj}[K(t, x) ; 0]=$ $\arg \min _{\xi \in K(t, x)}\|\xi\|$. This allows one to give a further formulation of a PDS, using Theorem 3.3, as: $\dot{x}=\operatorname{proj}\left[\{f(x)+g(t)\}+\mathcal{N}_{K}(x) ; 0\right]$. The last property $\dot{x} \in \mathcal{T}_{K}(x)$ makes a clear link with viability [62].

REMARK 13. Part of these equivalency results may be found in [308] and [62, Theorem p.217]. Comparing (2.31) (a) and (4.11) one sees that there is a similarity between PDS and SOSWP. As long as $K$ is convex closed, (3.8) (b) fits with maximal monotone DIs in Section 2.2: it is precisely this property that is used to prove that the solution is slow, relying on [123, Proposition 3.4]. When $K$ is a prox-regular set, the equivalence between (2.31) (a) and (3.8) (b) is shown in [300, Lemma 2.7] and [505, Proposition 5]. The minimal norm property of Theorem 3.3 holds in this case also [300, Proposition 2.10] [374, Theorem 3.2].

Remark 14. DIs of the form $\dot{x} \in-x+\operatorname{proj}[K ; x-\partial \phi(x)-\epsilon(t) \partial \xi(x)], x(0)=$ $x_{0} \in K$, are studied in [106] for the sake of optimization problems, with $\phi(\cdot)$ and $\xi(\cdot)$ convex continuous functions, $K \subseteq \mathbb{R}^{n}, \epsilon(t)>0$ and $\lim _{t \rightarrow+\infty} \epsilon(t)=0$. Still using the same tools we find that this can be rewritten equivalently as the implicit DI: $\dot{x} \in-x+f(x)-\mathcal{N}_{K}(\dot{x}+x)$ : this does not fit with maximal monotone DIs of 
Section 2.2, nor with the above projected systems. Their well-posedness with slow AC solutions is proved in [106, Theorem 1].

As alluded to in Remark 6, the right-hand side of (2.31) (a) makes sense for much larger classes of sets $K$ if one uses Clarke's cone $\mathcal{T}_{K}^{C}(x)$. What about Proposition 3.2 and Theorem 3.3, with non convex sets (e.g., prox-regular sets), and set-valued vector fields ? Notice that minimum norm solutions are present in DIs like (3.8) (b) with prox-regular sets $K$ [33, Theorem 4.2] [61, Theorem 10.1.1] (because the Bouligand tangent cone to prox-regular sets is LSC) [374].

3.9. HOSwP, complementarity systems, VIs. As for the SOSwP, there is a natural link between HOSwP in (2.9) and complementarity systems, due to the basic link between normal cones to polyhedral sets and complementarity conditions. It can be shown [6, Theorem 1] that $0 \leq z_{1}\left(t^{+}\right) \perp d \nu_{r}(\{t\}) \geq 0$ for all $t$, where $d \nu_{r}$ is the measure inside the set $\mathcal{N}_{\mathcal{T}_{\Phi_{m}}^{r-1}\left(Z_{r-1}\left(t^{-}\right)\right.}\left(z_{e, r}(t)\right)$ in $(2.9 \mathrm{c})$. This measure is in fact the measure part of the distributional multiplier $\lambda$ in (2.8). We see consequently that the HOSwP formalism incorporates complementarity between $\lambda$ and $z_{1}$, and can be considered as the right formulation of autonomous LCS (2.22) with arbitrary relative degree. Contrary to (2.22b) which is mathematically meaningful only outside state jump times, the HOSwP complementarity holds everywhere (and this has crucial consequences on its time-discretization via an event-capturing time-stepping scheme $[6$, Section 5]). The HOSwP is rewritten in a complementarity formalism as follows:

$$
\left\{\begin{array}{l}
\mathbf{D} x=A\{x\}+B \lambda \\
0 \leq w(t)=C x(t) \perp \chi_{r}(t) \geq 0, \quad \forall t \text { that is not an atom of } d \nu_{i}, 1 \leq i \leq r \\
0 \leq w\left(t^{+}\right) \perp d \nu_{r}(\{t\}) \geq 0, \forall t,
\end{array}\right.
$$

where $\mathbf{D}$ denotes distributional derivative, $d \nu_{i}$ is a measure, and $\chi_{r}(\cdot)$ are defined in Section 2.1.3. As shown in [6], the HOSwP can be formulated as a special kind of QVI, using the normal cone definition (A.2) in Section A.1.2. In other words, the inclusions in $(2.9 \mathrm{~b})$ are equivalent to: Find $z_{e, i}(t) \in T_{\Phi_{m}}^{i-1}\left(Z_{i-1}\left(t^{-}\right)\right)$such that

$$
\left\langle d z_{i}-z_{i+1}(t) d t, y-z_{e, i}(t)\right\rangle \geq 0 \text {, for all } y \in T_{\Phi_{m}}^{i-1}\left(Z_{i-1}\left(t^{-}\right)\right), \quad 1 \leq i \leq r-1,
$$

while the one in $(2.9 \mathrm{c})$ reads: Find $z_{e, r}(t) \in T_{\Phi_{m}}^{r-1}\left(Z_{r-1}\left(t^{-}\right)\right)$such that

$$
\left\langle d z_{r}-C A^{r-1} W^{-1} z(t) d t, y-z_{e, r}(t)\right\rangle_{C A^{r-1} B} \geq 0 \text {, for all } y \in T_{\Phi_{m}}^{r-1}\left(Z_{r-1}\left(t^{-}\right)\right) \text {. }
$$

Finally let us note that HOSwP are closely related to switching DAEs [132].

3.10. Relay systems and LCS. We have seen in Remark 12 that the relay system (2.35) with full rank $D$, can be recast into particular DIs which under some conditions are ODEs. As shown in [149, Chapter 7] [305, 397], relay systems (2.35) with $E_{3}=0$ and $E_{4}=0$, can be rewritten as an LCS as follows:

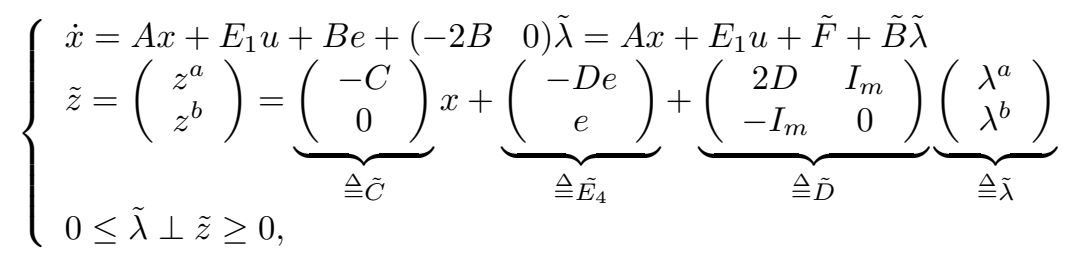

with $e=(1,1, \ldots, 1)^{T} \in \mathbb{R}^{m}, z^{a}$ and $z^{b} \in \mathbb{R}^{m}, \tilde{B} \in \mathbb{R}^{n \times 2 m}, \tilde{C} \in \mathbb{R}^{2 m \times n}$. This is a consequence of rewriting the set-valued signum function in a complementarity framework [130, Equation (5.122)]. Notice that (3.12) can be rewritten in a DI formalism as the $x$-dynamics in (3.2) (with added input $u=\tilde{E}_{4}$ ). 
Lemma 3.4. Suppose that $P B=C^{T}$ for some $P=P^{T} \succ 0$, and that $D \succcurlyeq 0$ in (2.35). Equivalently $\frac{P}{2} \tilde{B}=\tilde{C}^{T}$ and $\tilde{D} \succcurlyeq 0$.

The proof is led by simple calculations. It shows that the passivity properties of $(A, B, C, D)$ in $(2.35)$ transport to $(A, \tilde{B}, \tilde{C}, \tilde{D})$ in (3.12). The results in Section 3.4 yielding the FOSwP (3.6) do not apply to (3.12) since $\tilde{D}$ is never zero. Lemma 3.4 proves that $\left\{\operatorname{ker}\left(D+D^{T}\right) \subset \operatorname{ker}\left(P B-C^{T}\right)\right\}$ is equivalent to $\left\{\operatorname{ker}\left(\tilde{D}+\tilde{D}^{T}\right) \subset\right.$ $\left.\operatorname{ker}\left(\frac{P}{2} \tilde{B}-\tilde{C}^{T}\right)\right\}$, so the results in [524, Theorem 1, Corollary 2] can be used to analyse the relay LCS in (3.12). The fundamental operator for (3.12) is $x \mapsto \tilde{\lambda} \in(\tilde{D}+$ $\left.\partial \psi_{\mathbb{R}_{+}^{2 m}}\right)^{-1}\left(-\tilde{C} x-\tilde{E}_{4}\right)$. Conditions under which it is single valued Lipschitz continuous can be studied using [135, Propositions 3, Corollary 1]. We see that the relative degree between $\tilde{z}$ and $\tilde{\lambda}$ is zero since $\tilde{D}$ has full rank. However at most $\tilde{D} \succcurlyeq 0$ (even if $D \succ 0$ ) so that the LCP: $0 \leq \tilde{\lambda} \perp \tilde{z} \geq 0$ may have zero or several solutions, see [130, Theorem 5.7, Proposition 5.18, Example 5.11]. Nevertheless, it is found by inspection that this LCP is feasible (choose $\lambda^{a}=e$ and $\lambda^{b} \geq 0$ such that $\lambda^{b}>C x-D e$ ), thus it is solvable [210, Theorem 3.1.2], and its solution set is convex polyhedral with a minimum norm element [210, Theorem 3.1.7]. It can be verified that if $C x+D e \geq 0$, then $\lambda^{a}=e$ and $\lambda^{b}=C x+D e$ is a solution, while if $C x+D e \leq 0$ then $\lambda^{a}=\lambda^{b}=0$ is a solution. Thus if $m=1$ there is always one solution for any $x$. This short analysis shows that the LCS (3.12), may not be the best formulation for relay systems (see Remark 12 for the case $\left.D=D^{T} \succ 0\right)$.

REMARK 15. This is in fact a particular instance of relationship between a system where $\mathcal{F}(\cdot, \cdot, \cdot)$ in (1.1) is PWL (possibly set-valued with "vertical" branches), and complementarity systems. The resulting complementarity problem may not be an LCP however, but something more complex, see [125, Examples 4-7] and Section 3.15.

Interestingly enough the $\xi$-dynamics in (3.2) can also be recast into an LCS framework. Indeed the hyperbox $[-1,1]^{m}=\left\{\lambda \in \mathbb{R}^{m} \mid \bar{C} \lambda+e \geq 0\right\}, \bar{C} \in \mathbb{R}^{2 m \times m}$, $e=(1,1, \ldots, 1)^{T} \in \mathbb{R}^{m}$. The rows of $\bar{C}$ are of the form $\bar{C}_{2 i \bullet}=(0, \ldots, 0,-1,0, \ldots, 0)$ for $1 \leq i \leq m$, and $\bar{C}_{(2 i+1) \bullet}=(0, \ldots, 0,1,0, \ldots, 0)$ for $0 \leq i \leq m-1$. Then $N_{[-1,1]^{m}}(\lambda)=\left\{\zeta \in \mathbb{R}^{m} \mid \zeta=\sum_{i=1}^{2 m} \alpha_{i} \bar{C}_{i \bullet}^{T}, 0 \leq \alpha_{i} \perp \bar{C}_{i \bullet} \lambda+1 \geq 0\right\}$. Thus we get an equivalent representation of the system as:

$$
\left\{\begin{array}{l}
\dot{\xi}=A^{T} \xi+C^{T} \bar{C}^{T} \alpha \\
0 \leq \alpha \perp \bar{C} B^{T} \xi+\bar{C} D \bar{C}^{T} \alpha+e \geq 0 .
\end{array}\right.
$$

3.11. PWL systems and complementarity systems. In the first paragraph we show how to construct a PWL system (a subclass of the switching systems presented in Section 2.6) from a relative degree zero LCS. In the second paragraph, we show how to pass from a PWL system, to an intermediate nonlinear relay system, then an NLCS.

From LCS to PWL systems. Let us consider that $D$ in $(2.22)$ is a P-matrix. A basic complementarity theory result states that $\lambda$ is a PWL function of $C x+E_{3} u+E_{4}$ [210], i.e., $\lambda=f\left(C x+E_{3} u+E_{4}\right)$ with $f: \mathbb{R}^{m} \rightarrow \mathbb{R}^{m}$ Lipschitz continuous and PWL. Hence the LCS is the PWL system: $\dot{x}=A x+B f\left(C x+E_{3} u+E_{4}\right)+E_{1} u+E_{2}$. This means that the state space can be splitted in cells within which the vector field is constant (see [266, Example 7.4] for a planar example). A very simple illustrating example is: $\dot{x}=\lambda, 0 \leq \lambda \perp z=x+u+\lambda \geq 0$. If $x+u \geq 0$ then $\dot{x}=0$, if $x+u \leq 0$ then $\dot{x}=-x-u$. Here, the complementarity conditions define two cells partitioning $\mathbb{R}: x \geq-u$ and $x \leq-u$. Thus we see that the state space's cells depend on the control $u$ through the matrix $E_{3}$. They can also depend on $u$ through $C=C(u)$ as pointed out in Section 2.4.3, for instance we may set $0 \leq \lambda \perp z=u x+h \geq 0$. Such 
a dependency is rarely considered in the literature on PWL systems, though it may have crucial consequences on various issues (well-posedness, stability, stabilization, controllability, optimal control, etc). Another example is as follows:

EXAMPLE 5. Consider the complementarity condition in (2.22) with $m=2$, $C=I_{2}, E_{3}=I_{2}, E_{4}=0, u(t)=\left(u_{1}(t), u_{2}(t)\right)^{T}$, and $D=\left(\begin{array}{cc}2 & 1 \\ 1 & 1\end{array}\right)$ (so $D$ is a $P$-matrix). The following cases are in range.

1. $\lambda_{1}, \lambda_{2}>0$ and $x_{1}+2 \lambda_{1}+\lambda_{2}+u_{1}(t)=0, x_{2}+\lambda_{1}+\lambda_{2}+u_{2}(t)=0$. Then, the complementarity problem has the unique solution $\lambda=\left(x_{2}-x_{1}-u_{1}(t)+\right.$ $\left.u_{2}(t), x_{1}-2 x_{2}+u_{1}(t)-2 u_{2}(t)\right)^{T}$ if $x \in R_{1}(t)$ where $R_{1}(t)=\left\{x \in \mathbb{R}^{2} \mid x_{1}<\right.$ $\left.-u_{1}(t), u_{1}(t)-u_{2}(t)+x_{1}<x_{2}<\min \left(\frac{x_{1}-u_{1}(t)+2 u_{2}(t)}{2},-u_{2}(t)\right)\right\}$, and $R_{1}=$ $\left\{x \in \mathbb{R}^{2} \mid x_{1}<0, x_{2} \in\left(x_{1}, \frac{x_{1}}{2}\right)\right\}$ if $u_{1}(\cdot)=u_{2}(\cdot)=0$.

2. $\lambda_{1}>0, \lambda_{2}=0$ and $x_{1}+2 \lambda_{1}+u_{1}(t)=0, x_{2}+\lambda_{1}+u_{2}(t) \geq 0$. The complementarity problem has the unique solution $\lambda=\left(-\frac{x_{1}+u_{1}(t)}{2}, 0\right)^{T}$ if $x \in R_{2}(t)$ where $R_{2}(t)=\left\{x \in \mathbb{R}^{2} \mid x_{1}<-u_{1}(t), x_{2}>\frac{x_{1}}{2}+\frac{u_{1}(t)}{2}-u_{2}(t)\right\}$.

3. $\lambda_{1}=0, \lambda_{2}>0$ and $x_{1}+\lambda_{2}+u_{1}(t) \geq 0, x_{2}+\lambda_{2}+u_{2}(t)=0$. The complementarity problem has the unique solution $\lambda=\left(0,-x_{2}-u_{2}(t)\right)^{T}$ if $x \in R_{3}(t)$ where $R_{3}(t)=\left\{x \in \mathbb{R}^{2} \mid x_{2}<u_{2}(t), x_{1}>x_{2}+u_{2}(t)-u_{1}(t)\right\}$.

4. $\lambda_{1}=\lambda_{2}=0$ and $x_{1}+u_{1}(t) \geq 0, x_{2}+u_{2}(t) \geq 0$. The complementarity problem has the unique solution $\lambda=(0,0)^{T}$ if $x \in R_{4}(t)$ where $R_{4}(t)=\{x \in$ $\left.\mathbb{R}^{2} \mid x_{1}>-u_{1}(t), x_{2}>-u_{2}(t)\right\}$.

The mapping $x \mapsto \lambda(x, u(t))$ is PWL and continuous, the four cells are depicted in Figure 3.1 in the case $u(\cdot)=0$.

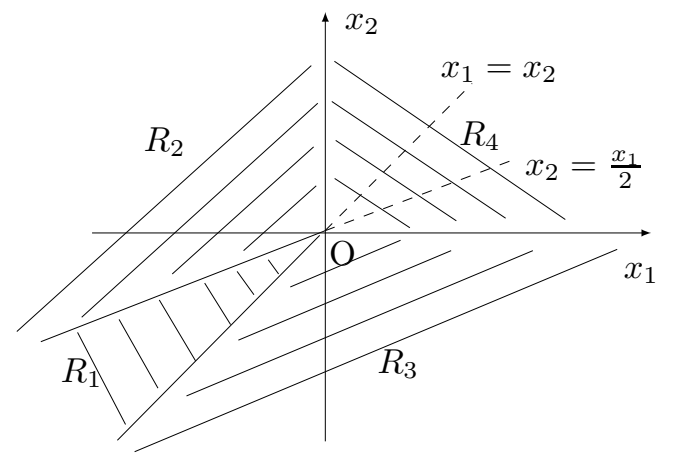

Fig. 3.1: The four regions in Example 5, with $u(\cdot)=0$.

$\rightsquigarrow$ Thus one can say that LCS with $D$ a P-matrix $\left(\Rightarrow r=(0, \ldots, 0)^{T}\right)$ naturally represent some classes of $P W L$ systems with continuous $P W L$ vector field.

It is also known that LCCS (see Section 2.4.3) with $K$ a polyhedral convex cone, are conewise PWL systems (i.e., a switching system where the cells $R_{i}=\left\{x \in \mathbb{R}^{n} \mid C_{i} x \geq\right.$ $0\}$ are convex cones, and $\left.f_{i}(x)=A_{i} x\right)[157,462]$.

From PWL systems to complementarity systems. The reverse question is less systematic, i.e., given a switching system as in Section 2.6, with possible vector field discontinuities, what is its representation as a complementarity system ? Some answers are given in [266, Section 11] [7, 226, 378, 149, 551, 156]. A systematic way to 
pass from a one-dimensional continuous PWL curve, to a complementarity problem, is provided in [551] (an example is treated in Section 3.15, see (3.19), see also [125, Examples 4-7]). The algorithm in [378, Section 2.6.3] allows one to pass from a continuous switching PWL vector field, to a function with nested absolute values, which can in turn be written via complementarity relations. The most intuitive idea, used in $[4,7,226]$ (see also [518]) is to associate with each cell, a step function which plays the role of a discrete Boolean function. Let us start with a simple case.

Proposition 3.5. Let $x \in \mathbb{R}^{n}, A_{1}$ and $A_{2} \in \mathbb{R}^{n \times n}$, $a_{1}$ and $a_{2} \in \mathbb{R}^{n}, H \in \mathbb{R}^{1 \times n}$, $h \in \mathbb{R}$. The dynamical complementarity system:

$$
\left\{\begin{array}{l}
\dot{x}=\frac{1}{2}\left(A_{1}+A_{2}\right) x+\frac{1}{2}\left[\left(A_{2}-A_{1}\right) x+a_{2}-a_{1}\right] \lambda+\frac{1}{2}\left(a_{1}+a_{2}\right) \\
H x+h=\lambda_{1}-\lambda_{2} \\
0 \leq 1+\lambda \perp \lambda_{1} \geq 0 \\
0 \leq 1-\lambda \perp \lambda_{2} \geq 0
\end{array}\right.
$$

is equivalent to the relay system: $\dot{x} \in \frac{1}{2}\left(A_{1}+A_{2}\right) x-\frac{1}{2}\left[\left(A_{2}-A_{1}\right) x+a_{2}-a_{1}\right] \operatorname{sgn}(H x+$ $h)+\frac{1}{2}\left(a_{1}+a_{2}\right)$, and they both represent the Filippov convexification (2.34) of the $P W L$ switching system: $\dot{x}=A_{1} x+a_{1}$ if $H x+h>0, \dot{x}=A_{2} x+a_{2}$ if $H x+h<0$. If $A_{1} x+a_{1}=A_{2} x+a_{2}$ when $H x+h=0$, then the switching system is equivalent to the LCS:

$$
\left\{\begin{array}{l}
\dot{x}=A_{1} x+a_{1}+B \lambda \\
0 \leq \lambda \perp z=H x+h+D \lambda \geq 0
\end{array}\right.
$$

with $D>0, A_{2}=A_{1}-\frac{1}{D} B H, a_{2}=a_{1}-\frac{1}{D} B h$.

The proof of the first equivalence relies on the fact that the set of complementarity conditions in (3.14), is equivalent to $-\lambda \in \operatorname{sgn}(H x+h)[130, \S 5.4 .4 .2]$, then checking that the right-hand side is the convex hull of the two vector fields when $H x+h=0$. The second equivalence follows by solving the LCP. We see that the relay system in Proposition 3.5 fits neither with (2.35), nor with (2.16), and is a kind of bilinear relay system. The extension of Proposition 3.5 to $p$ cells is proposed in [266] when $n=2$. An interesting issue is to analyse the case of switching systems with exogeneous switching times, obtained by setting $h=h(t)$ which could rule the switches between $H x(t)+$ $h\left(t^{-}\right)>0$ and $H x(t)+h\left(t^{+}\right)<0$. Various results on systems as in Proposition 3.5 can be found in [534] (relationships between Caratheodory and Filippov's solutions, uniqueness, Zeno behaviour).

Another class of PWL systems which can be easily transformed into complementarity systems, is made by max-min systems, i.e., systems involving $\max (0, \cdot)$ and $\min (0, \cdot)$ functions. Networks with unilateral interactions [408] belong to this class, and some hysteresis models use such operators as well [366]. Let us consider the next example taken from [408, Equation (4)]: $\dot{x}_{1}=\max \left(0, x_{3}-x_{1}\right), \dot{x}_{2}=\left(x_{1}-\right.$ $\left.x_{2}\right)+\min \left(0, x_{3}-x_{2}\right), \dot{x}_{3}=\max \left(0, x_{1}-x_{3}\right)$. From the equivalence $z=\max (0, u) \Leftrightarrow$ $\{z=\lambda$ and $0 \leq \lambda \perp \lambda-u \geq 0\}[587,378]$, and $z=\min (0, u)=-\max (0,-u)$, one can rewrite equivalently the system as the LCS:

$$
\left\{\begin{array}{c}
\dot{x}=\left(\begin{array}{ccc}
0 & 0 & 0 \\
1 & -1 & 0 \\
0 & 0 & 0
\end{array}\right) x+\left(\begin{array}{ccc}
1 & 0 & 0 \\
0 & -1 & 0 \\
0 & 0 & 1
\end{array}\right) \lambda \\
0 \leq \lambda \perp \lambda+\underbrace{\left(\begin{array}{ccc}
1 & 0 & -1 \\
0 & -1 & 1 \\
-1 & 0 & 1
\end{array}\right)}_{\triangleq_{C}} x \geq 0 .
\end{array}\right.
$$


This LCS has $D=I_{3}$, and is thus trivially well-posed, being a PWL system with continuous Lipschtiz vector field, as in Example 5. We can rewrite (3.16) as another ODE with Lipschitz vector field using (A.6) and (B.1): $\lambda=\operatorname{proj}\left[\mathbb{R}_{+}^{3} ;-C x\right]$. The case $z=\max (0, u, v)=\max (\max (0, u), v)$ is treated similarly. Define $z_{1}=\max (0, u) \Longleftrightarrow$ $\left\{z_{1}=\lambda_{1}\right.$ and $\left.0 \leq \lambda_{1} \perp \lambda_{1}-u \geq 0\right\}$. Then $z=\max \left(z_{1}, v\right) \Leftrightarrow z=v+\max \left(0, z_{1}-v\right)$ $\Longleftrightarrow\left\{z=v+\lambda\right.$ and $\left.0 \leq \lambda \perp \lambda-z_{1}+v \geq 0\right\}$. Thus a network with the dynamics [408, Equation (20)]: $\dot{x}_{1}=\max \left(0, x_{3}-x_{1}\right)+\max \left(0, x_{2}-x_{1}\right), \dot{x}_{2}=\max \left(0, x_{1}-\right.$ $\left.x_{2}\right), \dot{x}_{3}=\max \left(0, x_{1}-x_{3}, x_{2}-x_{3}\right)$, can also be recast into the LCS framework. The so-called (static, see Remark 8) backlash hysteresis contains functions like $y=$ $\max (u, \min (v, w))=\max (u,-\max (-v,-w))[333$, Section 6], which can be rewritten as $y=w-\lambda_{1}+\lambda_{2}, 0 \leq \lambda_{1} \perp \lambda_{1}+v-w \geq 0,0 \leq \lambda_{2} \perp-\lambda_{1}+\lambda_{2}-u+w \geq 0$, indicating a strong link with LCS.

REMARK 16. Is the relay framework amenable for such min-max systems? One idea, that we follow again below, is to set $z=u \frac{1+\operatorname{sgn}(u)}{2}$, which yields the desired result that $z=\max (0, u)$. Direct extension towards $\max (u, v)=u \frac{1+\operatorname{sgn}(u-v)}{2}+v \frac{1-\operatorname{sgn}(u-v)}{2}$ seems more problematic, since at $u=v$ it yields $z \in[0,2 u]$ or $[2 u, 0]$. Hence the resulting right-hand side is strictly larger than $\max (u, v)$ in $u-v=0$.

Consider now that the state space is separated into four regions (cells) with four different vector fields $f_{i}(x):\left(R_{1}\right)$ with $f_{1}(x): h_{1}(x)<0, h_{2}(x)<0,\left(R_{2}\right)$ with $f_{2}(x)$ : $h_{1}(x)<0, h_{2}(x)>0,\left(R_{3}\right)$ with $f_{3}(x): h_{1}(x)>0, h_{2}(x)<0,\left(R_{4}\right)$ with $f_{4}(x)$ : $h_{1}(x)>0, h_{2}(x)>0$. An LCS with complementarity conditions as in Example 5, defines such regions. Following [4, Section 7.1], let us rewrite the systems as:

$$
\begin{aligned}
\dot{x} \in & \frac{1-\operatorname{sgn}\left(h_{1}(x)\right)}{2} \frac{1-\operatorname{sgn}\left(h_{2}(x)\right)}{2} f_{1}(x)+\frac{1-\operatorname{sgn}\left(h_{1}(x)\right)}{2} \frac{1+\operatorname{sgn}\left(h_{2}(x)\right)}{2} f_{2}(x) \\
& +\frac{1+\operatorname{sgn}\left(h_{1}(x)\right)}{2} \frac{1-\operatorname{sgn}\left(h_{2}(x)\right)}{2} f_{3}(x)+\frac{1+\operatorname{sgn}\left(h_{1}(x)\right)}{2} \frac{1+\operatorname{sgn}\left(h_{2}(x)\right)}{2} f_{4}(x),
\end{aligned}
$$

where $\operatorname{sgn}(\cdot)$ is the set-valued signum function. Such a procedure can be extended to $m$ regions [226, Equation (4.2)], then each term on the right-hand side involves the product of $m$ step functions $\sigma^{+}(x) \triangleq \frac{1+\operatorname{sgn}(x)}{2}$ and $\sigma^{-}(x) \triangleq \frac{1-\operatorname{sgn}(x)}{2}$. Due to the products of step functions in the right-hand side of (3.17), the mere mathematical meaning of such a DI is not straighforward. We can associate with each step function a set of complementarity conditions as in (3.14). However we see that this will necessarily involve products of multipliers like $\lambda$ in the dynamics: what we obtain is far from a gentle LCS, it is an NLCS (2.24) where the vector field in (2.24a) is nonlinear in $\lambda$. Thus giving a precise mathematical meaning to the vector field on the boundaries between the cells $R_{i}$ is mandatory, especially if these surfaces are attractive of codimension $\geq 2$ and give rise to sliding modes (as defined by Filippov) which are undetermined in the sense that Filippov's convexification does not yield a unique vector field on such surfaces (it is noteworthy that the right-hand side of the DI in (3.17), is Filippov's convexification (2.34) everywhere except on the codimension 2 surface $\Sigma \triangleq\left\{x \in \mathbb{R}^{n} \mid h_{1}(x)=h_{2}(x)=0\right\}$ (see for instance [226] for an analysis of this issue, based on a suitable regularisation inside $\Sigma$ which allows one to choose a particular selection of the set-valued right-hand side). Let us briefly review the approach taken in [7] to relate (3.17) to complementarity systems. It consists first of interpreting the DI (3.17) as an Aizerman-Pyatnitskii's DI (AP-DI) [44, 45], instead of a Filippov's DI (an idea also used in [404] who in passing study the relationships between a class of nonlinear relay systems and DIs). Let $f(x, u)$ be given, with $f: \mathbb{R}^{n} \times \mathbb{R}^{p} \rightarrow \mathbb{R}^{n}$ continuous in both $x$ and $u, u: \mathbb{R}^{n} \rightarrow \mathbb{R}^{p}$ is discontinuous. At points of discontinuities, it is assumed that $u_{i} \in U_{i}(x)$ and $u_{j} \in U_{j}(x), i \neq j$, vary 
independently. The right-hand side of an AP-DI is defined as $G(x) \triangleq\left\{y \in \mathbb{R}^{n} \mid y=\right.$ $\left.f(x, u), u_{i} \in U_{i}(x), 1 \leq i \leq p\right\}$. In general $G(x)$ is not convex, and it is usually tempting to use instead $H(x)=\overline{\operatorname{conv}}(G(x))$. For (3.17) the AP-DI right-hand side is constructed as follows. Let us rewrite (3.17) as: $\dot{x} \in \sum_{1}^{4} f_{i}(x) g_{i}\left(\sigma^{ \pm}\left(h_{1}(x)\right), \sigma^{ \pm}\left(h_{2}(x)\right)\right.$, where $g_{1}\left(\sigma^{ \pm}\left(h_{1}(x)\right), \sigma^{ \pm}\left(h_{2}(x)\right) \triangleq \sigma^{-}\left(h_{1}(x)\right) \sigma^{-}\left(h_{2}(x)\right), g_{2}\left(\sigma^{ \pm}\left(h_{1}(x)\right), \sigma^{ \pm}\left(h_{2}(x)\right) \triangleq\right.\right.$ $\sigma^{-}\left(h_{1}(x)\right) \sigma^{+}\left(h_{2}(x)\right)$, and so on. Notice that $\sigma^{-}(x)=1-\sigma^{+}(x)$, so that $\dot{x} \in$ $f(x)+\sum_{1}^{4} f_{i}(x) g_{i}^{+}\left(\sigma^{+}\left(h_{1}(x)\right), \sigma^{+}\left(h_{2}(x)\right)\right.$ for suitable $g_{i}^{+}(\cdot)$ and $f(x)$. Thus letting $\sigma_{i} \in[0,1], i=1,2$, the AP-DI right-hand side is equal to $f(x)+\sum_{1}^{4} f_{i}(x) g_{i}^{+}\left(\sigma_{1}, \sigma_{2}\right)$, replacing every occurence of $\sigma^{+}\left(h_{1}(x)\right)$ by $\sigma_{1}$ and every occurence of $\sigma^{+}\left(h_{2}(x)\right)$ by $\sigma_{2}$ : $G(x)=\left\{z \in \mathbb{R}^{n} \mid z=f(x)+\sum_{1}^{4} f_{i}(x) g_{i}^{+}\left(\sigma_{1}, \sigma_{2}\right), \sigma_{1} \in \sigma^{+}\left(h_{1}(x)\right), \sigma_{2} \in \sigma^{+}\left(h_{2}(x)\right)\right\}$. Under some conditions on the functions $f_{i}(\cdot)$ and $g_{i}^{+}(\cdot)$ (which are satisfied for the models studied in [7]) one has $G(x)=\overline{\operatorname{conv}}(G(x))=F(x)$ with $F(x)$ in (2.34), and the existence of AC solutions follows. Our point is however here to exhibit an NLCS from (3.17). First of all let us notice that for any scalars $\sigma$ and $h: \sigma \in \frac{1+\operatorname{sgn}(h)}{2} \Leftrightarrow$ $2 \sigma-1 \in \operatorname{sgn}(h) \Leftrightarrow h \in \mathcal{N}_{[-1,1]}(2 \sigma-1) \Leftrightarrow h \in \mathcal{N}_{[0,1]}(\sigma)$, where we used material from Section A.2.4. We can therefore rewrite equivalently the DI in (3.17) as:

$$
\left\{\begin{array} { l } 
{ \dot { x } = f ( x ) + \sum _ { 1 } ^ { 4 } f _ { i } ( x ) g _ { i } ^ { + } ( \sigma _ { 1 } , \sigma _ { 2 } ) } \\
{ h _ { i } ( x ) \in \mathcal { N } _ { [ 0 , 1 ] } ( \sigma _ { i } ) , \quad i = 1 , 2 }
\end{array} \Leftrightarrow \left\{\begin{array}{l}
\dot{x}=f(x)+\sum_{1}^{4} f_{i}(x) g_{i}^{+}\left(\sigma_{1}, \sigma_{2}\right) \\
h_{i}(x)=\xi_{1, i}-\xi_{2, i} \\
0 \leq \xi_{1, i} \perp \sigma_{i} \geq 0 \\
0 \leq \xi_{2, i} \perp-\sigma_{i}+1 \geq 0, \quad i=1,2 .
\end{array}\right.\right.
$$

Letting $\lambda \triangleq\left(\sigma_{1}, \sigma_{2}, \xi_{1,1}, \xi_{2,1}, \xi_{1,2}, \xi_{2,2}\right)^{T}$, the dynamics in the right-hand side of (3.18) is an NLCS with an added equality of the form $H(x, \lambda)=0$ in $(2.24)$, which we could name a mixed NLCS. This idea can be used to recast the operator $[\omega]_{z}^{+}=\omega$ if $\omega>0$ and $z>0$, and $[\omega]_{z}^{+}=0$ otherwise, into NLCS: the cells $R_{i}, 1 \leq i \leq 4$, are the quadrants of $\mathbb{R}^{2}$, and $f_{1}(\omega, z)=\omega$ while $f_{i}(\omega, z)=0$ for $2 \leq i \leq 4$, hence $[\omega]_{z}^{+}=\sigma^{+}(\omega) \sigma^{-}(z) \omega$ and is the Filippov's convexification of the switching PWL vector field. Such operators are used in [244, 268, 190, 302] for the sake of designing dynamical systems whose equilibria are the solutions of optimization problems (we saw in Section 2.5 the relation with projected dynamics and this operator), in [579] for instant model predictive control.

$\rightsquigarrow$ An open question is how to relate LCS with D a P-matrix, and DIs as in (3.17). In other words, under which conditions can one construct an LCS with a D that is a P-matrix, starting from the generic formulation in (3.17), with arbitrary number of cells?

3.12. Complementarity systems and nonsmooth DAEs. It is well known that a complementarity condition between two scalar variables: $0 \leq \lambda \perp z \geq 0$ is equivalent to $\phi(\lambda, z)=0$ where $\phi: \mathbb{R}^{2} \rightarrow \mathbb{R}$ is any complementarity function [4, Definition 12.53]. The most well-known C-function is $\phi(\lambda, z)=\min (\lambda, z)$, but there are many other ones [4, p.386] [240, Section 1.5.1], some of which are nondifferentiable, and some are differentiable (everywhere). Extending this to $\lambda \in \mathbb{R}^{m}$ is easy, getting a vector C-function $\Phi(\lambda, z)$. Then any complementarity system may be seen as a DAE: $\dot{x}=f(x, \lambda, u, t)$ subject to $\Phi(\lambda, z)=0$. The topic of nonsmooth DAEs is important in process and chemical engineering applications [516], and its relationships with complementarity systems still deserves further studies.

3.13. Maximal monotone DIs and convex processes. A so-called convex process, as introduced in [491, Section 39], is a DI: $\dot{x} \in A(x)$, with $A: \mathbb{R}^{n} \rightarrow \mathbb{R}^{m}$, 
such that $\operatorname{Graph}(A)$ is a convex cone: $A(\alpha x)=\alpha A(x)$ for all $\alpha>0$ and $x \in \operatorname{dom}(A)$, $A\left(x_{1}\right)+A\left(x_{2}\right) \subset A\left(x_{1}+x_{2}\right)$ for all $x_{1}, x_{2} \in \operatorname{dom}(A)$ (clearly, normal cones and signum set-valued functions do not satisfy the latter). Typical examples are [513, 274]: $A_{1}(x)=M x+K, M$ a constant matrix, $K \subset \mathbb{R}^{n}$ a convex cone, of $A_{2}(x)=M x+K_{1}$ if $x \in K_{2}, A_{2}(x)=\emptyset$ if $x \notin K_{2}, K_{1}, K_{2}$ convex cones. If $\operatorname{dom}(A)=\mathbb{R}^{n}$ and the graph is closed, the process is said strict closed convex, in which case $A(\cdot)$ is a Lipschitzian set-valued map [513, Theorem 2.12]. Consider $A_{2}(\cdot)$ with $K_{1}=0$ and $K_{2}=\mathbb{R}_{+}$, $M=m \in \mathbb{R}$. Consider also $\dot{x} \in A_{3}(x)=-\mathcal{N}_{\mathbb{R}_{+}}(x)+m x$, which belongs to the class of systems considered in Section 2.2. One has $\operatorname{Graph}\left(A_{2}\right)=\{(x, y) \mid y=m x, x \geq$ $0\} \subset \operatorname{Graph}\left(A_{3}\right)=\{(x, y) \mid y=m x, x \geq 0\} \cup\{(0, y), y \geq 0\}$; however, $\operatorname{Graph}\left(A_{3}\right)$ is non convex and hence the process $\dot{x} \in A_{3}(x)$ is not convex. Convex processes are also in general non monotone. We infer that convex processes do not belong to the class of set-valued systems analysed in this article. The concept of monotone process also exists [490], that is not to be confused with DIs with maximal monotone in Section 2.2 .

3.14. Mechanical systems with impacts and Filippov's DIs. Simple mechanical systems with a single unilateral constraint (or constraints orthogonal in the kinetic metric, see Section 5.4.2) and dissipative impacts with Newton kinematic law [130], can be recast into a class of DIs which may be interpreted through Filippov's approach [590, 329, 332]. This is known as the Zhuravlev-Ivanov transformation, see [130, Section 1.4.3]. Since the obtained DI involves the set-valued signum function, it can also be recast into complementarity systems, using the material in Section 3.10. Thus one passes from a system with AC positions and LBV velocities (a measure DI), to a system with $\mathrm{AC}$ solutions. This is one of the many nonsmooth state transformations, which allow one to decrease the system's nonsmoothness (see [130, Section 1.4] for a short survey).

3.15. Hysteresis operators, DIs, complementarity systems, FOSwP. Many hysteresis operators such as rheological models made of assemblies of springs, dashpots and Coulomb dry friction elements, lend themselves to a representation with maximal monotone operators [558] [130, Section 2.3]. This has been shown in the case of complex assemblies in [91, 90, 87]. Hence, when inserted in dynamics, they give rise to DIs as in (2.16). Following the procedure in [551], the description of the graphs in Figure 2.3 (a) (solid arrows), (b) and (c) is systematic. For instance, the square has the representation:

$$
\left\{\begin{array}{c}
\left(\begin{array}{l}
u \\
y
\end{array}\right)=\left(\begin{array}{l}
-\epsilon \\
-1
\end{array}\right)+M \lambda_{1}+N \lambda_{2} \\
0 \leq \lambda_{1} \perp \lambda_{2} \geq 0, \quad E\left(\lambda_{1}-\lambda_{2}\right)=e
\end{array}\right.
$$

with $M=\left(\begin{array}{cccccc}-1 & 0 & 0 & 0 & 0 & 0 \\ 0 & 0 & 0 & 0 & 0 & 0\end{array}\right), N=\left(\begin{array}{cccccc}0 & 2 \epsilon & 0 & -2 \epsilon & 0 & 1 \\ 2 & 0 & 0 & 0 & -2 & -2\end{array}\right), E=$ $\left(\begin{array}{cccccc}1 & -1 & 0 & 0 & 0 & 0 \\ 1 & 0 & -1 & 0 & 0 & 0 \\ 1 & 0 & 0 & -1 & 0 & 0 \\ 1 & 0 & 0 & 0 & -1 & 0 \\ 1 & 0 & 0 & 0 & 0 & -1\end{array}\right), e=\left(\begin{array}{lllll}1 & 2 & 3 & 4 & 5\end{array}\right)^{T}$, which is a mixed LCP. It yields when inserted in a dynamics, a system as in (2.25). The zig-zag operator in Figure 2.3 (b) can also be written via complementarity [378, equation (2.26)]. This is 
quite different from the completed relay operator (dashed arrows in Figure 2.3 (a)), see [558, Section 1.4.1]. In [71, Theorem 2.1] [557, Chapter III], it is shown that the so-called play operator of hysteresis in Figure 2.3 (a), with parallelogram instead of square shape, fits within the FOSwP: $\dot{y} \in-\mathcal{N}_{[-\rho+u(t), \rho+u(t)]}(y)$, and with timevarying LCS (using the normal cone linearization cone, Section A.1.2). Let us now deal with Duhem model. It is an exercise to transform the Duhem operator dynamics (2.36) into the NLCS (2.24):

$$
\left\{\begin{array}{l}
\dot{x}_{1}=f_{1}\left(x_{1}, x_{2}\right) \lambda_{1}+f_{2}\left(x_{1}, x_{2}\right) \lambda_{2} \\
\dot{x}_{2}=v \\
0 \leq \lambda_{1} \perp z_{1}=\lambda_{1}-v \geq 0 \\
0 \leq \lambda_{2} \perp z_{2}=\lambda_{2}+v \geq 0
\end{array}\right.
$$

where $v=\dot{u}$, hence $x_{2}(\cdot)=u(\cdot)$ if $x_{2}(0)=u(0)$. Due to the form of the complementarity conditions, $\lambda_{1}$ and $\lambda_{2}$ are piecewise continuous, Lipschitz functions of $v$, so that (3.20) is a kind of nonlinear system, PWL in the input. The Duhem hysteresis operator (2.36) can be modified to a constrained version, and thus can be written as a VI, equivalently as a DI into the normal cone $\mathcal{N}_{\overline{\mathcal{S}}(u)}(y)$ to a set $\overline{\mathcal{S}}(u)=\left[\gamma_{r}(u), \gamma_{l}(u)\right]$, $\gamma_{r}(\cdot)$ and $\gamma_{l}(\cdot)$ are continuous and non decreasing, $\gamma_{r}(u) \leq \gamma_{l}(u)$ for all $u[558$, Section 1.2.1]. Then $y(t) \in \overline{\mathcal{S}}(u(t))$ for all $t \geq 0$. One then obtains from [558, Equation $(1.26)]$ :

$$
\left\{\begin{array}{l}
\dot{x}_{1}-f_{1}\left(x_{1}, x_{2}\right) \lambda_{1}-f_{2}\left(x_{1}, x_{2}\right) \lambda_{2} \in-\mathcal{N}_{\overline{\mathcal{S}}\left(x_{2}\right)}\left(x_{1}\right) \\
\dot{x}_{2}=v \\
0 \leq \lambda_{1} \perp z_{1}=\lambda_{1}-v \geq 0 \\
0 \leq \lambda_{2} \perp z_{2}=\lambda_{2}+v \geq 0 .
\end{array}\right.
$$

The system in (3.21) is a mixture between a FOSwP with state dependent set $\overline{\mathcal{S}}(x)$, and complementarity conditions. The complementarity conditions make a perturbation $f(t, x, v)$ in $(2.2 \mathrm{~b})$, which is PWL in $v$. The multifunctions in Figure 2.3 (a) and (b) can be represented with complementarity relations, since their graph is PWL [551]. The case of the delayed relay hysteresis in Figure 2.3 (c) is more complex, because the vertical segments cannot be reached by any pair $(u, y)$. Further studies on hysteresis (the rate independent play operator) and the FOSwP are in $[353,354,362,361]$, where the so-called stop operator $\mathcal{X}: W^{1,1}\left([0, T] ; \mathbb{R}^{n}\right) \rightarrow W^{1,1}\left([0, T] ; \mathbb{R}^{n}\right), u \mapsto x$, has the dynamics $\dot{x}+\mathcal{N}_{\mathcal{S}}(x) \ni \dot{u}, x(t) \in \mathcal{S}$ for all $t \geq 0$; it has Lipschitz continuous solutions $x(u)$.

Let us end this section with the double inclusion [557, Section VI.3]: $\left(\partial \psi_{K_{1}}\right)^{-1}(\dot{x})+$ $\partial \psi_{K_{2}}(x) \ni u(t), K_{1} \subseteq \mathbb{R}^{n}$ and $K_{2} \subseteq \mathbb{R}^{n}$ closed convex sets. This is rewritten as $\dot{x} \in \partial \psi_{K_{1}}\left(-\partial \psi_{K_{2}}(x)\right)+\partial \psi_{K_{1}}(u)$, which is close to a degenerate FOSwP [369, 371]. Assume the sets are cones. Using (B.1) and after few manipulations, one obtains the cone LCS: $\dot{x}=\eta(x)+\gamma(u), K_{1} \ni u \perp-\gamma(u) \in K_{1}^{\star}, K_{2} \ni x \perp \mu(x) \in K_{2}^{\star}$, $K_{1} \ni \mu(x) \perp-\eta(x) \in K_{1}^{\star}$. Letting $\lambda=(\gamma, \mu, \eta)^{T}$, one sees that the matrix $D$ in the variable $z$ of the complementarity conditions as in (2.22), is singular for such systems.

3.16. Conclusions. All the introduced formalisms are closely related one with each other. Even in case of equivalence, each framework may possess its own advantages and drawbacks. For instance complementarity systems usually lend themselves very well to numerical simulation, because of efficient LCP solvers [4, 240]. They can also easily handle classes of PWL systems with time-varying, or control-dependent cells (see Example 5 in Section 3.11). It is also easy to consider control-dependent 
constraint-sets in the FOSwP. Normal cones are convenient for stability issues, being maximal monotone operators. Maximal monotone operators encompass classes of infinite dimensional systems, while such extensions are less clear for other formalisms. It is possible that the field of application of each formalism, is -and will remain- one of the most important challenges for our systems. The question of generality of a mathematical model, is crucial: what is the degree of generality, above which a formalism becomes useless, because it is intractable for analysis, control, numerical simulation? Most of the above formalisms could be embedded into:

$$
P(t, x) \dot{x} \in F(t, x)-G(t, x)\left(g(t, x)+\mathcal{M}_{t, x}\right)^{-1}(h(t, x))
$$

with a matrix $P$ constant and full-rank, $F(\cdot)$ may be set-valued, $\mathcal{M}_{t, x}(\cdot)$ maximal monotone for each $t$ and each $x$, or with a relaxed property $\left(\mathcal{M}_{t}(\cdot)=\partial \varphi_{t}(\cdot)\right.$ with $\varphi_{t}(\cdot)$ non convex function). One could also consider switching systems where the dynamics in each cell is a DI as (3.22). The case of varying singular matrix $P(t, x)$, possibly set-valued, is also worth investigating, and its relationships with switching DAE with state-dependent switching times, as well as mixed LCS, should be studied. See (4.7) for a motivating example involving $P(x, \dot{x})$. The usefulness of such extensions has to be justified either by an application field, or by the ability to bring new theoretical results. It seems that systems with time delays have been analyzed only in the framework of FOSwP, with delay in the perturbation $f(\cdot, \cdot)$. Finally as alluded to in Section 2.1.5, singular FOSwP, MLCS, switching DAEs, singular DIs as in (2.18) and their relationships, whose analysis remains largely open, deserve attention.

4. Applications. Let us describe some applications of the foregoing mathematical formalisms. In view of the above developments, all these models can be interpreted as in (1.1).

4.1. Electrical circuits. Electrical circuits have been studied in a set-valued framework in various articles and books $[93,256,507,552,152,21,2,11,135,9,19$, $27,378,546,377,12,278]$. The dynamics of electrical circuits with ideal, set-valued components (diodes, Zener diodes, transistors) can be recast into LCS as in (2.22) (a fact known since at least [377] building on earlier works by van Bokhoven and Leenaerts, see [378] and references therein), or as FOSwP (a fact noticed for the first time in [125, Section IV]), or as DVIs. Roughly speaking, circuits with ideal diodes are passive LCS with relative degree between $z$ and $\lambda$ equal to zero or one (from Theorem D.2 they have a transfer matrix with total index 1 , and a supply rate $\lambda^{T} z=0$ ). Let us illustrate this with the circuits depicted in Figures 4.1 and 4.2. Roughly speaking, the set-valued part comes from the voltage/current law of the diodes, while the smooth 
part of the dynamics represents $R L C$ dynamics.

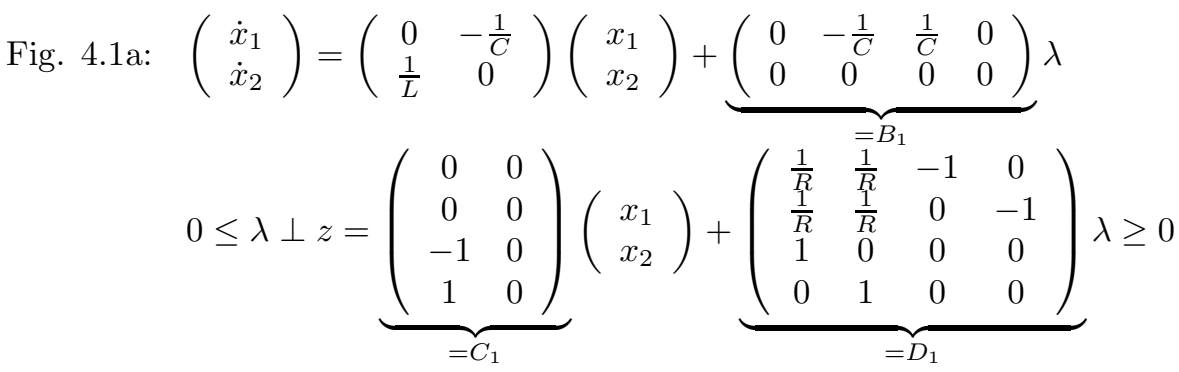

$x_{1}=v_{L}, x_{2}=i_{L}, \lambda=\left(-v_{D R 1},-v_{D F 2}, i_{D F 1}, i_{D R 2}\right)^{T}$
$y=\left(i_{D R 1}, i_{D F 2},-v_{D F 1},-v_{D R 2}\right)^{T}$.

Fig. 4.1b: $\quad\left(\begin{array}{c}\dot{x}_{1} \\ \dot{x}_{2} \\ \dot{x}_{3}\end{array}\right)=\left(\begin{array}{ccc}\frac{-2}{R C} & \frac{1}{R C} & 0 \\ \frac{1}{R C} & \frac{-2}{R C} & \frac{1}{R} \\ 0 & 0 & 0\end{array}\right)\left(\begin{array}{c}x_{1} \\ x_{2} \\ x_{3}\end{array}\right)+\left(\begin{array}{cc}0 & \frac{1}{R C} \\ 0 & 0 \\ \frac{1}{C} & 0\end{array}\right) \lambda$

$$
\begin{aligned}
& 0 \leq \lambda \perp z=\left(\begin{array}{ccc}
0 & 0 & 1 \\
\frac{-2}{R C} & \frac{1}{R C} & 0
\end{array}\right)\left(\begin{array}{l}
x_{1} \\
x_{2} \\
x_{3}
\end{array}\right)+\underbrace{\left(\begin{array}{cc}
0 & 0 \\
0 & \frac{1}{R}
\end{array}\right)}_{=D_{2}} \lambda \geq 0 \\
& x_{1}(t)=\int_{0}^{t} i_{1}(s) d s, x_{2}(t)=\int_{0}^{t} i_{2}(s) d s, x_{3}=v_{2} \\
& \lambda_{1}=-i_{3}, \lambda_{2}=v_{1} .
\end{aligned}
$$

Fig. 4.1c: $\quad \dot{x}=\frac{-R}{L} x+\frac{1}{L} \lambda$

$$
\begin{aligned}
& 0 \leq \lambda \perp z=x-i(t) \geq 0 \\
& \lambda=v .
\end{aligned}
$$

Fig. 4.2a: $\left(\begin{array}{c}\dot{x}_{1} \\ \dot{x}_{2}\end{array}\right)=\left(\begin{array}{cc}0 & 1 \\ \frac{-1}{L C} & \frac{-R}{L}\end{array}\right)\left(\begin{array}{c}x_{1} \\ x_{2}\end{array}\right)+\underbrace{\left(\begin{array}{c}0 \\ \frac{-1}{L}\end{array}\right)}_{=B_{4}} \lambda+\left(\begin{array}{c}0 \\ \frac{1}{L}\end{array}\right) u(t)$

$0 \leq \lambda \perp z=\underbrace{\left(\begin{array}{ll}0 & -1\end{array}\right)}_{=C_{4}}\left(\begin{array}{l}x_{1} \\ x_{2}\end{array}\right) \geq 0$

$x_{1}(t)=\int_{0}^{t} i(s) d s, x_{2}=i, \lambda=v$.

Fig. 4.2b: $\quad\left(\begin{array}{c}\dot{x}_{1} \\ \dot{x}_{2}\end{array}\right)=\left(\begin{array}{cc}\frac{-1}{R C} & 1 \\ \frac{-1}{L C} & 0\end{array}\right)\left(\begin{array}{c}x_{1} \\ x_{2}\end{array}\right)+\left(\begin{array}{c}\frac{-1}{R} \\ \frac{-1}{L}\end{array}\right) \lambda+\left(\begin{array}{cc}0 & \frac{1}{R} \\ \frac{-1}{L} & \frac{1}{L}\end{array}\right) u(t)$

$$
\begin{aligned}
& 0 \leq \lambda \perp z=\left(\begin{array}{ll}
\frac{1}{R C} & -1
\end{array}\right)\left(\begin{array}{l}
x_{1} \\
x_{2}
\end{array}\right)+\underbrace{\frac{1}{R}}_{=D_{5}} \lambda+\left(\begin{array}{ll}
0 & \frac{-1}{R}
\end{array}\right) u(t) \geq 0 \\
& x_{1}(t)=\int_{0}^{t} i_{1}(s) d s, x_{2}=i_{2}, \lambda=v, u^{T}=\left(u_{1}, u_{2}\right) .
\end{aligned}
$$


Fig. 4.1d: $\quad\left(\begin{array}{c}\dot{x}_{1} \\ \dot{x}_{2}\end{array}\right)=\left(\begin{array}{cc}0 & 1 \\ \frac{-1}{L C} & \frac{-R}{L}\end{array}\right)\left(\begin{array}{c}x_{1} \\ x_{2}\end{array}\right)+\left(\begin{array}{c}0 \\ \frac{-1}{L}\end{array}\right) u(t)+\left(\begin{array}{c}0 \\ \frac{1}{L}\end{array}\right) v$

$v \in-\partial g\left(x_{2}\right)$

$$
x_{1}(t)=\int_{0}^{t} i(s) d s, x_{2}=i \text {. }
$$

The first five circuits' dynamics can be recast into (2.22) with feedthrough matrices satisfying: $D_{1} \succcurlyeq 0, D_{1}$ full rank, $D_{1} \neq D_{1}^{T}$ and $D_{1} \neq-D_{1}^{T}, D_{2}=D_{2}^{T} \succcurlyeq 0, D_{3}=$ $D_{4}=0, D_{5}=D_{5}^{T} \succ 0$. Thus they may be analysed with different tools, starting with the developments in Section 3 (for instance, the circuit in Figure 4.1 (b) fits within the framework of [135] and (2.17), the one in Figure 4.1 (c) can be recast as a FOSwP, while the one in Figure 4.2 (b) is an ODE with Lipschitz right-hand side). The circuit in Figure 4.1 (d) has two ideal Zener diodes mounted in series, each one satisfying $v_{1} \in \partial g_{1}\left(i_{1}\right)$ and $v_{2} \in \partial g_{2}\left(i_{2}\right)$, with $g_{i}(x)=U_{i} x$ if $x \geq 0, g_{i}(x)=-V_{i} x$ if $x \leq 0, U_{i}>0, V_{i}>0$. Thus the assembly has the voltage/current set-valued law $v \in \partial g(i)$ with $g(x)=\left(U_{1}+V_{2}\right) x$ if $x \geq 0, g(x)=-\left(U_{2}+V_{1}\right) x$ if $x \leq 0$. The function $g(\cdot)$ being proper convex continuous, the dynamics of the last circuit fits within (2.16) with $\mathcal{F}(t, x)=\mathcal{F}(x)$. Zinc oxide varistors also possess a voltage/current law of the same type. The circuit in Figure $4.2 \mathrm{a}$ also fits within this class of maximal monotone DIs, since $P B_{4}=C_{4}^{T}$ for any $P=\operatorname{diag}\left(p_{11}, L\right)$ with $p_{11}>0$ (the change of state variable $\xi=R x$, pointed out in Section 3.4, can be applied to obtain a DI as (3.6) with fixed set $\Phi)$. Circuits with both Zener and ideal diodes can also be put under the same umbrella, see [2, Fig. 1.16] [278, Fig. 5.2], with a set-valued right-hand side mixing subdifferentials of indicator functions and relay-like functions. It is noteworthy that all the presented circuits, satisfy $\left(A_{i}, B_{i}, C_{i}, D_{i}\right)$ is passive (see Section $\mathrm{D}$ ), hence the framework in [151] may apply provided $\left(\begin{array}{c}B_{i} \\ D_{i}+D_{i}^{T}\end{array}\right)$ has full column rank [151, Assumption 3.1]. The above circuits naturally yield, using the material in Section 3.4 , FOSwP where the control input acts in both the vector field and in the set $\mathcal{S}$, as in $(3.6)$.

These circuits are academic examples, further examples can be found in [139, Example 3.152] [2, Equation (5.64)] [155, Equation (21)] for circuits with $D \neq 0, D+D^{T}=0$ and $P B=C^{T}$. If one considers electronic components with more complex PWL voltage/current laws (like tunnel diodes which have a characteristic similar to the zigzag in Figure 2.3 (b), memristors, Schmidt triggers with characteristic as in Figure 2.3 (c), operation amplifiers that yield signum multifunction or its inverse $\mathcal{N}_{[-1,1]}(\cdot)$, negative resistance converters, etc), then extensions of LCS as in (2.25) may have to be considered, see [125, Example 7], see also Cuk, buck, and delta-sigma converters in $[2$, Chapters 7, 8]. A big difference with usual control systems where one normally considers independent inputs (thus $m \leq n$ and $B$ has full column rank) is that here $\lambda$ has the dimension of the number of nonsmooth components in the circuit, so that one may have in practical examples $m \gg n$ (in (4.1) one has $n=2$ and $m=4$ ). The case of nonlinear resistors $R(i)$ (varistors) and nonlinear inductances $L(i)$ is of interest, because it allows to illustrate a non trivial $P(x)$ in (3.22). Consider the circuit in (4.5), with $R=R(i)$ (see [479] for an example with $R(i)=R_{0}+R_{1} i^{2}+R_{2} i^{4}$ ), $L=L(i)$ [1, pp.11-273-11-274], so that the voltage at the bounds of the resistor is given by $R(i) i$, while the inductance voltage is $L(i) \frac{d i}{d t}$. The dynamics of this circuit 
with such varistor is written as:

$$
\left\{\begin{array}{l}
R\left(\dot{x}_{1}, x_{2}\right) \dot{x}_{1}-R\left(\dot{x}_{1}, x_{2}\right) x_{2}=u_{2}(t)-\frac{1}{c} x_{1}-\lambda \\
L\left(x_{2}\right) \dot{x}_{2}=-\frac{1}{C} x_{1}-\lambda+u_{1}(t)-u_{2}(t) \\
0 \leq \lambda \perp-\dot{x}_{1} \geq 0
\end{array}\right.
$$

This gives rise to a system as in (3.22) with $P=P(x, \dot{x})$. The dynamics in (4.7) is a differential algebraic implicit LCS (more complex that the ZOSwP seen in Section 3.5). The circuit in (4.2) yields also this type of dynamics. It is noteworthhy that even in the absence of nonsmooth ingredients, ODEs with powers of the derivatives require specific analysis [243]. Capacitors with polynomial nonlinearities can also be considered [379, 243], which may possess also a hysteretic voltage/charge law [409], hence adding another source of nonsmoothness in the dynamics.

Let $U_{\mathrm{ns}} \in \mathbb{R}^{m-1}$ and $I_{\mathrm{ns}} \in \mathbb{R}^{m}$ be vectors which collect the voltages and the currents at the $m$ ports of the nonsmooth elements of a circuit. A general formulation of the nonsmooth elements is as follows [2, Chapter 4]:

$$
\left\{\begin{array}{l}
y=g_{\mathrm{ns}}\left(I_{\mathrm{ns}}, U_{\mathrm{ns}}, x, t\right) \\
0=h_{\mathrm{ns}}\left(I_{\mathrm{ns}}, U_{\mathrm{ns}}, x, t\right) \\
0 \in G(x, y)+F(x, y)
\end{array}\right.
$$

with $F: \mathbb{R}^{n \times n} \rightrightarrows \mathbb{R}^{m}$ a set-valued mapping, $G: \mathbb{R}^{n \times n} \rightarrow \mathbb{R}^{n}$ is a continuously differentiable mapping. The dynamics of circuits with nonsmooth elements is usually obtained after a so-called Modified Nodal Analysis and insertion of (4.8), and results in a complex nonsmooth dynamical system mixing equalities and inclusions [2, Equation (4.3)]. Clearly the tools which are reviewed in this article are not sufficient to analyse nonsmooth circuits in their full generality. But they can prove to be quite useful for a first analysis or control design.

REMARK 17. Let us notice in passing that dissipativity of $(A, B, C, D)$ guarantees under mild assumptions that the LCP $0 \leq \lambda \perp C x+D \lambda \geq 0$, possesses a solution along the dynamical system's solutions, even if $D \succcurlyeq 0$ as in (4.1). This follows from [29, Theorem 1] which guarantees the maximal montonicity of the system's right-hand side, or using [151] (see Proposition 2.1). Then further properties hold [130, Theorem 5.7, Proposition 5.18] [210]. Notice also that when $D+D^{T}=0$ and $D \neq 0$, the change of state variable used to get (3.6) no longer applies.

REMARK 18. What is the motivation to use such nonsmooth models for electronic components? As shown in [407], exponential models of diodes may lead to extremely stiff ODEs for which the Newton-Raphson algorithm fails (see [2, Chapter 7] for comparisons between several integrators, including commercial ones based on SPICE models, or using enumeration of all modes). Some circuits may also have trajectories with sliding modes, in such a case the set-valued nonsmooth models can be simulated in a very efficient way with suitable event-capturing time-stepping methods. Finally set-valued nonsmooth models may simplify the study and help analysing some issues (for instance, discontinuity with respect to initial data, or non uniqueness of solutions, may just mean high sensitivity with respect to parameters and initial data which could not be easily detected and understood using smooth stiff models). The above set-valued nonsmooth models also yield compact formalisms which avoid designers to carry useless ballast, a fact which makes the analysis easier when a very large number $m$ of nonsmooth elements is considered, implying a number of modes increasing exponentially with $m$.

\subsection{Multibody mechanical systems with unilateral contact.}




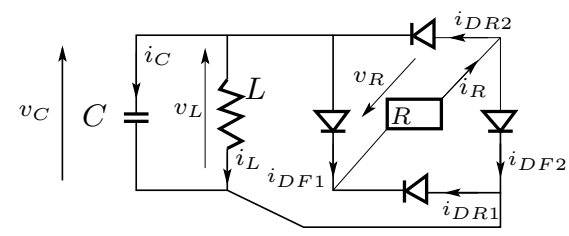

(a)

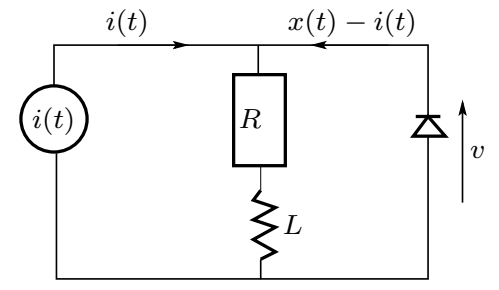

(c)

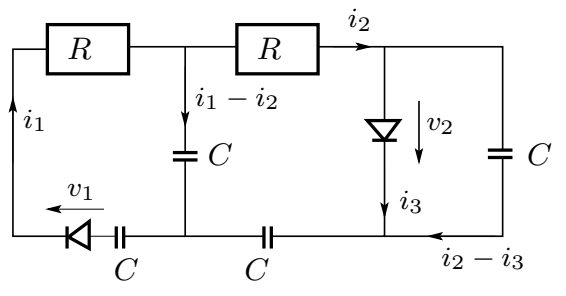

(b)

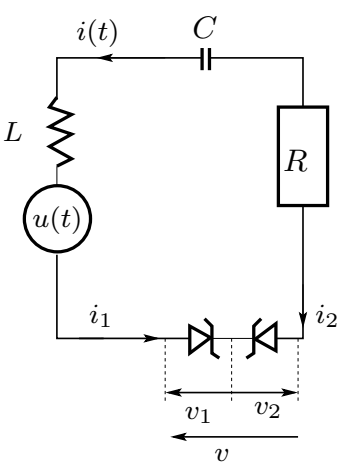

(d)

Fig. 4.1: RLC circuits with ideal and Zener diodes, current and voltage sources.

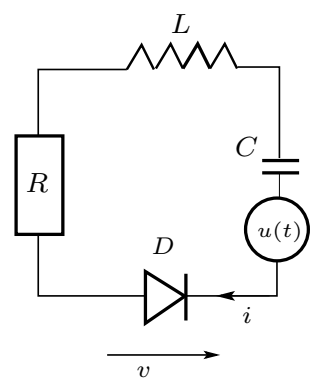

(a)

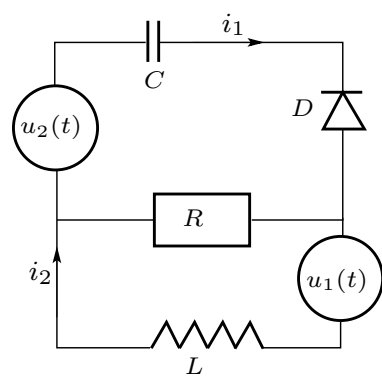

(b)

Fig. 4.2: RLC circuits with an ideal diode and voltage sources.

4.2.1. Perfectly rigid frictionless bodies. Multibody mechanical systems dynamics can be written either in the Newton-Euler, or in the Lagrange formalisms. In any case, unilateral constraints represent the signed distances between bodies at potential contact/impact points. They are introduced with gap functions $h_{i}(q) \geq 0$, $1 \leq i \leq m_{u}$, where $m_{u}$ is the number of unilateral constraints. In most of real (industrial) applications, bilateral constraints $g_{i}(q)=0,1 \leq i \leq m_{b}$, are also present, here we shall assume that coordinates have been reduced in a suitable way. Contact forces associated with the constraints are introduced through Lagrange multipliers $\lambda_{\mathrm{n}, i}$. In many instances, a contact model based on the complementarity conditions 
$h_{i}(q) \lambda_{\mathrm{n}, i}=0$, together with non-negativity $\lambda_{\mathrm{n}, i} \geq 0$, is used [130, 478, 272, 442,385]. This is a contact model that stems from very general and natural physical assumptions, see [130, Section 5.4.1]. This is compactly written as $0 \leq \lambda_{\mathrm{n}} \perp h(q) \geq 0$, which holds componentwise due to non-negativity. Therefore we obtain a complementarity Lagrangian system with perfect unilateral constraints [381, 130]:

$$
\left\{\begin{array}{l}
M(q) \ddot{q}+C(q, \dot{q}) \dot{q}+g(q)=E(q) u+\nabla h(q) \lambda_{\mathrm{n}} \\
0 \leq \lambda_{\mathrm{n}} \perp z_{1}=h(q) \geq 0 \\
\text { Impact mapping, }
\end{array}\right.
$$

where the generalized force $\nabla h(q) \lambda_{\mathrm{n}}$ stems from the principle of virtual work [130, $478,272]$. Since the complementarity conditions are equivalently written as $\lambda_{\mathrm{n}} \in$ $-\partial \psi_{\mathbb{R}_{+}^{m_{u}}}(h(q))$, we get $\nabla h(q) \lambda_{\mathrm{n}} \in-\nabla h(q) \partial \psi_{\mathbb{R}_{+}^{m_{u}}}(h(q))$. By the nonsmooth analysis chain rule (see Section A.2.3, or [492, Theorem 10.6] [143, Theorem B.3, Corollary B.2]), we obtain $\nabla h(q) \lambda_{\mathrm{n}} \in-\partial\left(\psi_{\mathbb{R}_{+}^{m_{u}}} \circ h\right)(q)=-\partial \psi_{\Phi}(q)$, with $\Phi=\left\{q \in \mathbb{R}^{\frac{n}{2}} \mid h(q) \geq\right.$ $0\}$. Thus (4.9) (a) (b) is equivalent to the inclusion $M(q) \ddot{q}+C(q, \dot{q}) \dot{q}+g(q)-E(q) u \in$ $-\mathcal{N}_{\Phi}(q)$.

REMARK 19. We have led the calculations abruptly, however some assumptions have to be made so that this all makes sense. Firstly let us assume that the gap functions are continuously differentiable, and with $\nabla h_{i}(q) \neq 0$ for all $i$ such that $h_{i}(q)=0$ (active constraints). From a mechanical point of view, this means that the contact force at contact $i$ influences the motion. For the differentiation of $\left(\psi_{\mathbb{R}_{+}^{m_{u}}} \circ h\right)$, we may apply Proposition A.4 in Section A.2.3. We may thus check the sufficient condition (A.12), which boils down to $\operatorname{Im}\left(\nabla h(q)^{T}\right)+\mathbb{R}_{-}\left(\mathbb{R}_{+}^{m_{u}}-h(q)\right)=\mathbb{R}^{m_{u}}$ : the independency of the $m_{u}$ constraints guarantees this condition. We may also consider the affine function $\mathbb{R}^{n} \rightarrow \mathbb{R}^{m}: w \mapsto h(q)+\nabla h(q)^{T} w$ : if its range cannot be separated from $\mathbb{R}_{+}^{m_{u}}$ the condition (A.10) holds. Once this is done, one has to determine which type of normal cone we are working with. Under suitable conditions on $\nabla h(q)$, the set $\Phi$ may be prox-regular so that the Fréchet, basic, Clarke and proximal normal cones are identical (see Section A.2.1). The normal cone may then be any of these ones. Under a constraint qualification like the MFCQ, these normal cones admit a representation using the active constraints gradients and multipliers (here the contact forces) satisfying complementarity conditions, see Section A.1.2. Then $\mathcal{N}_{\Phi}(q)=\mathcal{N}_{\Phi}^{h}(q)$. We suppose that this is the case in the following, since it allows us to link the complementarity formalism in (4.9) and the differential inclusion formalism of the SOSwP.

Moreau's SOSwP goes a step further, and imposes an inclusion as in (2.6c):

$$
-M(q) d v-C(q, v) v d t-g(q) d t+E(q) u d t \in \mathcal{N}_{V(q)}\left(v_{e}\right) \subseteq \mathcal{N}_{\Phi}(q),
$$

which under the conditions discussed in Remark 19, is equivalent to (4.9) (a) (b) (c) with Newton's impact law in a complementarity form and a unique global restitution coefficient $[273,130]$. Using (B.1), one sees that $\lambda \in-\mathcal{N}_{V(q)}\left(v_{e}\right)$ is equivalent to $V(q) \ni v_{e} \perp \lambda \in(V(q))^{\star}=-\mathcal{N}(q)$ (compare with (3.5): now the cones depend on $q$, so that the SOSwP normal cone reduces the index, in a sense), where $V(q)$ and $\mathcal{N}(q)$ are both closed convex polyhedral cones (see Section 2.1.2). At an impact time the dynamics (4.10) becomes algebraic: $M(q)\left(v\left(t^{+}\right)-v\left(t^{-}\right)\right) \in-\mathcal{N}_{V(q)}\left(v_{e}\right)$, that is a generalized equation close to (A.6). Relatively simple manipulations using (A.6) allow one to obtain that (compare with (2.13))

$$
v\left(t^{+}\right)=-e v\left(t^{-}\right)+(1+e) \operatorname{proj}_{M(q)}\left[V(q) ; v\left(t^{-}\right)\right] .
$$


Several other formulations of the velocity reinitialization can be derived [130, Equations (5.60) (5.61)], in a way similar to (2.30). The expression in (4.11) prepares well for calculations. As we will see below, it can be formulated using a complementarity problem, that can be solved numerically.

REMARK 20. Why not going a step further, and imposing an inclusion in the set $-\mathcal{N}_{T_{V(q)}(\dot{q})}(\ddot{q})$ in (4.10)? This is one way to extend Gauss' principle to systems with unilateral constraints [130, Remark 5.1]. But this is not amenable at impact times.

We have seen in foregoing sections that the relative degree between the complementary variables, is a crucial system's parameter. Consequently, let us examine the relative degree $\bar{r} \in \mathbb{N}^{m_{u}}$ between $\lambda_{\mathrm{n}}$ and $z_{1}=h(q)$ in (4.9) (a) (b). Let $M(q) \succ 0$. Differentiating we get $\frac{d z_{1}}{d t}=\nabla h(q)^{T} \dot{q}$, so that:

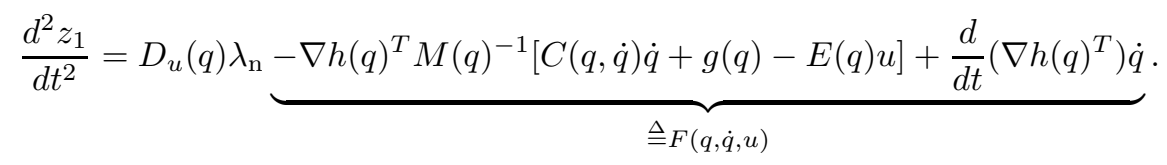

The matrix $D_{u}(q) \triangleq \nabla h(q)^{T} M(q)^{-1} \nabla h(q) \succcurlyeq 0$ is the Delassus' matrix ${ }^{8}$, which corresponds in Control Theory to the input-ouput decoupling matrix of the input-ouput linearization process. If the constraints are independent, then $D_{u}(q) \succ 0$ and the vector relative degree $\bar{r}=(2,2, \ldots, 2)^{T} \in \mathbb{R}^{m_{u}}$. Obtaining the canonical form (2.8) is not straightforward for nonlinear systems (in particular the zero-dynamics in (2.8) (c)), and requires conditions which we do not recall here [145]. In the linear invariant case, the existence of a relative degree is necessary and sufficient for the existence of a change of state that brings the system into (2.8). Let us assume that a global diffeomorphic change of coordinates exists $(q, \dot{q})=Z\left(z_{1}, z_{2}, \xi\right), z_{1}=h(q), z_{2}=\nabla h(q)^{T} \dot{q}$, which allows us to rewrite (4.9) (a) with $\lambda_{\mathrm{n}}$ as the input and $z_{1}$ as the output in the canonical form:

$$
\left\{\begin{array}{l}
\left(\text { a) } \dot{z}_{1}=z_{2}\right. \\
(b) \dot{z}_{2}=\tilde{D}_{u}\left(z_{1}, \zeta\right) \lambda_{\mathrm{n}}+\tilde{F}\left(z_{1}, z_{2}, \zeta, u\right) \\
(c) \dot{\zeta}=\Xi\left(\zeta, z_{1}\right) \\
(d) 0 \leq \lambda_{\mathrm{n}} \perp z_{1} \geq 0,
\end{array}\right.
$$

where $\tilde{F}(\cdot)=F \circ Z(\cdot), \tilde{D}_{u}(\cdot)=D_{u} \circ Z$. The dynamics in (4.13) (c) is the zero-dynamics of the input/output system. The canonical form (4.13) shows that at an impact, one part of the state (including velocity components) is continuous, since $\zeta(\cdot)$ and $z_{1}$ are continuous. In the new coordinates, Moreau's set is equal to $\mathcal{N}_{V(q)}\left(z_{2, e}\right) \subseteq \mathcal{N}_{\mathbb{R}_{+}^{m}}\left(z_{1}\right)$, $V(q)=\tilde{V}\left(z_{1}\right)=\left\{v \in \mathbb{R}^{n} \mid v_{i} \geq 0, i \in \mathcal{I}\left(z_{1}\right)\right\}$.

EXAMPLE 6. Let us consider a flexible mechanical system made of five masses related by springs and subject to two unilateral constraints, as in Figure 4.3 (a). To simplify the presentation we assume that all five masses are equal to $M>0$, and all springs stiffnesses are equal to $k>0$. The masses coordinates are $q_{i}, 1 \leq i \leq 5$, thus $n=10$, and the two unilateral constraints defining the normal form "outputs" are $q_{5} \geq 0, q_{3} \geq 0$, thus $m_{u}=2, \bar{r}=(2,2)^{T}$. The dynamics is given in the original and

\footnotetext{
${ }^{8}$ In the honour of Etienne Delassus, who was the first to systematically investigate existence and uniqueness of solutions to contact complementarity problems [221].
} 
canonical forms by:

$$
\left\{\begin{array} { l } 
{ M \ddot { q } _ { 1 } = k ( q _ { 4 } - q _ { 1 } ) + k ( q _ { 2 } - q _ { 1 } ) } \\
{ M \ddot { q } _ { 2 } = k ( q _ { 3 } - q _ { 2 } ) + k ( q _ { 1 } - q _ { 2 } ) } \\
{ M \ddot { q } _ { 3 } = k ( q _ { 2 } - q _ { 3 } ) + \lambda _ { 2 } } \\
{ M \ddot { q } _ { 4 } = k ( q _ { 1 } - q _ { 4 } ) + k ( q _ { 5 } - q _ { 4 } ) } \\
{ M \ddot { q } _ { 5 } = k ( q _ { 4 } - q _ { 5 } ) + \lambda _ { 1 } . }
\end{array} \quad \text { and } \quad \left\{\begin{array}{l}
\dot{z}_{1}^{1}=z_{2}^{1} \\
\dot{z}_{2}^{1}=\frac{k}{M}\left(\xi_{5}-z_{1}^{1}\right)+\frac{\lambda_{1}}{M} \\
\dot{z}_{1}^{2}=z_{2}^{2} \\
\dot{z}_{2}^{2}=\frac{k}{M}\left(\xi_{3}-z_{1}^{2}\right)+\frac{\lambda_{2}}{M} \\
\dot{\zeta}=A_{\zeta} \zeta+B_{\zeta} z_{1},
\end{array}\right.\right.
$$

where $z_{1}^{1}=q_{5}, z_{1}^{2}=q_{3}, z_{1}=\left(z_{1}^{1}, z_{1}^{2}\right)^{T} \in \mathbb{R}^{2}, z_{2}=\left(z_{2}^{1}, z_{2}^{2}\right)^{T} \in \mathbb{R}^{2}, C A B=I_{2}$, $\zeta=\left(q_{1}, \dot{q}_{1}, q_{2}, \dot{q}_{2}, q_{4}, \dot{q}_{4}\right)^{T} \in \mathbb{R}^{6}, A_{\zeta}=\left(\begin{array}{cccccc}0 & 1 & 0 & 0 & 0 & 0 \\ -\frac{2 k}{M} & 0 & \frac{k}{M} & 0 & \frac{k}{M} & 0 \\ 0 & 0 & 0 & 1 & 0 & 0 \\ \frac{k}{M} & 0 & -\frac{2 k}{M} & 0 & 0 & 0 \\ 0 & 0 & 0 & 0 & 0 & 1 \\ \frac{k}{M} & 0 & 0 & 0 & -\frac{2 k}{M} & 0\end{array}\right), B_{\zeta}=$ $\left(\begin{array}{cccccc}0 & 0 & 0 & 0 & 0 & \frac{k}{M} \\ 0 & 0 & 0 & \frac{k}{M} & 0 & 0\end{array}\right)^{T}$. The zero dynamics contains the dynamics of masses 1, 2, 4, connected by linear springs.

Linear mechanical systems satisfy the input/output passivity constraint $P B=C^{T}$, $P=P^{T} \succ 0$, only if the output is the velocity $\dot{z}_{1}$. Thus the manipulations done in Section 3.4 to get (3.6), cannot be performed since the complementarity involves $z_{1}$. Moreau's set in (4.10) allows one to cope with this issue, with a kind of index (or relative degree) reduction from 2 to 1 , for given $q$ (but, the complete system still has a relative degree 2 ).

Moreau's set and complementarity. Let us investigate the link between Moreau's set $\mathcal{N}_{V(q)}\left(v_{e}\right)$, and complementarity. First it follows from (B.1) that the inclusion into Moreau's set can be equivalently rewritten as the CCP: $V(q) \ni v_{e} \perp \nabla h(q) \lambda_{\mathrm{n}} \in$ $-(V(q))^{\circ}=-\mathcal{N}_{\Phi}^{h}(q)$. Let us now analyze deeper the consequences of imposing the inclusion of the dynamics in Moreau's set. Using (4.12) we have in the right-hand side of (4.13) (b) the inclusion: $-D_{u}(q) \lambda_{\mathrm{n}} \in-\nabla h(q)^{T} M(q)^{-1} \mathcal{N}_{V(q)}\left(v_{e}\right)$. Using the material in Section A.1.2 (first the definition of the tangent cone linearization cone, then the definition of the normal cone to a convex polyhedral set), we can calculate that $\mathcal{N}_{V(q)}\left(v_{e}\right)=\left\{v \in \mathbb{R}^{n} \mid v=-\sum_{i \in \mathcal{K}\left(v_{e}\right)} \lambda_{i} \nabla d_{i}\left(v_{e}\right), \lambda_{i} \geq 0\right\}$, where $\mathcal{K}\left(v_{e}\right) \triangleq\{i \in$ $\left.\mathcal{I}(q) \mid d_{i}\left(v_{e}\right) \triangleq v_{e}^{T} \nabla h_{i}(q)=0\right\} \subseteq \mathcal{I}(q)$ is the set of position active constraints which are also active at the velocity level. We therefore obtain $-\nabla h(q)^{T} M(q)^{-1} \mathcal{N}_{V(q)}\left(v_{e}\right)=$ $\sum_{i \in \mathcal{K}\left(v_{e}\right)} \lambda_{i} \nabla h(q)^{T} M(q)^{-1} \nabla h_{i}(q)$. From the definition of the index sets, it follows $\sum_{i \in \mathcal{K}\left(v_{e}\right)} \lambda_{i} \nabla h(q)^{T} M(q)^{-1} \nabla h_{i}(q)=D_{u}(q) \lambda_{\mathrm{n}}$ with: $\lambda_{\mathrm{n}, i}=0$ if $h_{i}(q)>0$, and if $h_{i}(q)=0$, then $0 \leq \lambda_{\mathrm{n}, i} \perp \nabla h_{i}(q)^{T} v_{e} \geq 0$. Thus Moreau's set implies a two-stage process for the calculation of the contact forces multiplier. This is illustrated in Figure 4.3 (b) with the two index sets for different velocities and positions. This, in passing, shows how the impact law in the SOSwP is related to the classical Newton's impact law. Outside impacts $v_{e}=v \stackrel{\text { a.e. }}{=} \dot{q}$. At an impact time $t$ it can be shown [273] [130, Proposition 5.15] using similar tools that $0 \leq p_{\mathrm{n}, i}(t) \perp h_{i}(q(t))^{T} v_{e}(t) \geq 0$, $i \in \mathcal{I}(q(t))$, where $p_{\mathrm{n}, i}(t)$ is the density of the measure $\lambda_{\mathrm{n}, i}$ with respect to the Dirac measure: $\lambda_{\mathrm{n}, i}=p_{\mathrm{n}, i}(t) \delta_{t}$. Using the impact dynamics, a complementarity problem is constructed from which the post-impact velocity and the impulse can be calculated. We see that the SOSwP implies $\left(z_{1}^{i}, z_{2}^{i}\right) \succcurlyeq 0$, and that the index sets $\mathcal{I}(q)$ and $\mathcal{K}\left(v_{e}\right)$ reflect this lexicographical inequality. 

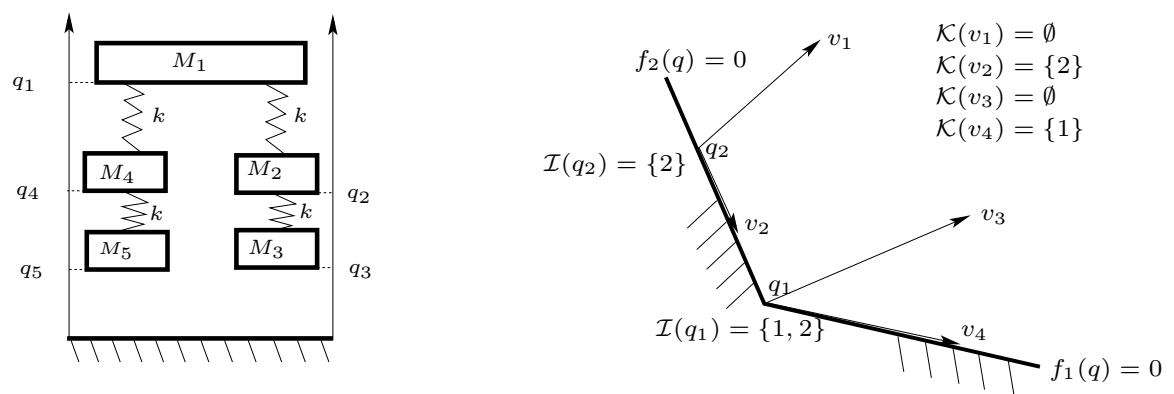

Fig. 4.3: (a) Mechanical system with two constraints, (b) SOSwP.

$\rightsquigarrow$ It follows from Sections 3.4 and 4.2.1, that an important characteristic of the FOSwP is that its underlying dynamics has a relative degree $\bar{r}$ with $r$ equal to 0 or 1 , while the SOSWP has a relative degree with $r=2$. The HOSwP has relative degree with $r \geq 3$. The relative degree $\bar{r}$ strongly influences the space of solutions, see Section 5 .

REMARK 21. Most of systems of interest in practice and industry, are subjected to both unilateral and bilateral constraints, that is one should add to (4.9b) a set of equality constraints $g(q, t)=0$. Strangely enough mixed unilateral-bilateral constraints have not been much studied in the multibody system dynamics literature. Bilateral constraints have the effect of distorting the contact LCP matrix, see for instance [130, Propositon 5.8] [129, 109].

REMARK 22. The unilateral constraints may be time-varying $h(q, t)$. Also the control input u may not enter the dynamics as in (4.9a), but through the gap functions $h(q)$, see [130, Example 1.6]. This last case has not been considered yet in the literature on complementarity mechanical systems, though it occurs commonly in circuits (see Section 4.1).

Moreau's SOSwP has been formulated for finitely represented admissible sets $\Phi$, with differentiable gap functions. What about the case when $\Phi$ cannot be represented this way, like in the presence of reentrant corners as the set $K_{6}$ in Figure A.1 (b)? One may think of replacing $V(q)$ by Clarke's tangent cone $\mathcal{T}_{\Phi}^{C}(q)$, which is closed convex (see Section A.1.2), so that $\mathcal{N}_{\mathcal{T}_{\Phi}^{C}(q)}\left(v_{e}\right)$ makes sense with the normal cone of convex analysis. This issue has been studied in [271].

To end this section, let us point out that the $\mathrm{SOSwP}$ in (4.10) is recast naturally in setvalued nonlinear Lur'e systems, since the smooth Lagrangian dynamics defines (under a suitable assumption on the potential energy) a passive subsystem $\xi \mapsto v_{e}$, with $\xi \in-\mathcal{N}_{V(q)}\left(v_{e}\right)$, in negative feedback interconnection with the set-valued operator $v_{e} \mapsto-\xi$. See [130, Section 7.5.3] [139, Section 6.8.2] [126].

4.2.2. Compliant contact/impact models. Consider the simple case of a one degree-of-freedom mechanical system, with a unilateral compliant contact. The contact model is assumed to possess its own dynamics and state $\xi$ (think of a springdashpot Kelvin-Voigt model). Let the signed distance between the mass and the spring-dashpot system be denoted as $h(q, \xi)$. The system's dynamics is:

$$
\left\{\begin{array}{l}
m \ddot{q}=u+F(\xi) \\
\dot{\xi}=g(\xi) \\
0 \leq z=h(q, \xi) \perp F(\xi) \geq 0,
\end{array}\right.
$$




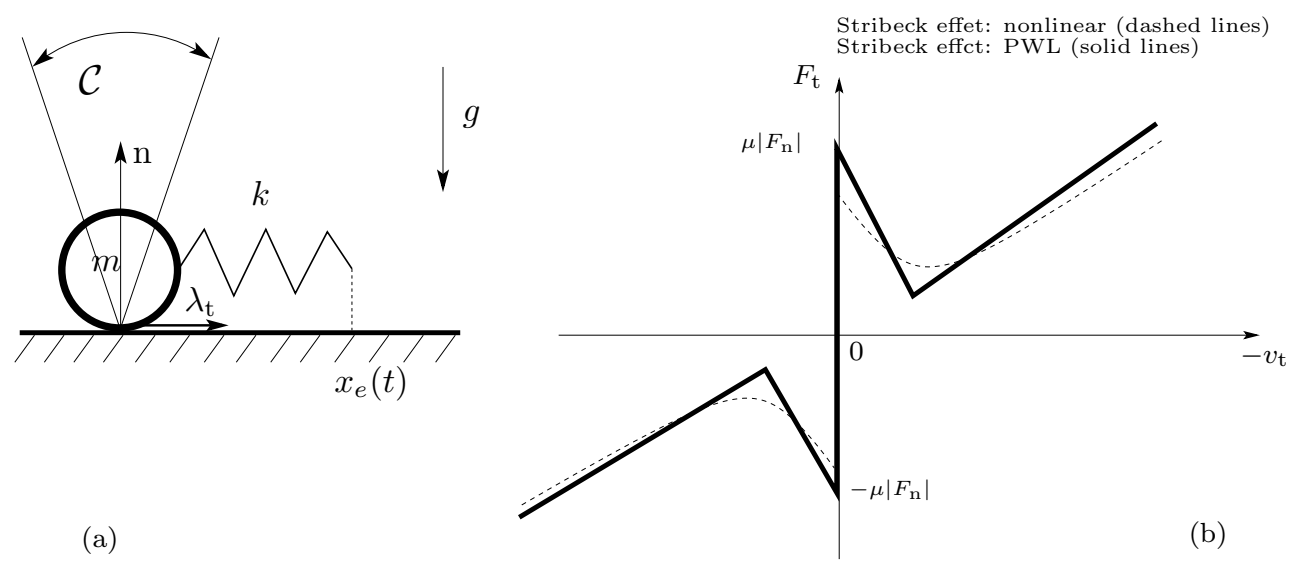

Fig. 4.4: (a) Mass + spring with Coulomb's friction; (b) Set-valued friction with Stribeck effect

for some $g(\cdot)$, which simply states that when the body and the obstacle are not in contact, then the non-negative interaction force vanishes. If the interaction force is positive, then the two systems touch each other with $h(q, \xi)=0$. A complete analysis of the linear spring-dashpot model is made in $[130, \S 2.1]$, where it is shown that the linear spring-dashpot model fits within (2.22) with a relative degree 1. Rheological models made of assemblies of springs, dashpots, and dry friction elements, are widely used for contact/impact modeling, see [541] [130, Chapter 2] and references therein. Such models may have complex enough structure, even if the springs and dashpots are linear (like Masing, Persoz' gephyroidal models [87, 91, 92, 88, 89], see also [352, Figure 4]). Consequently their well-posedness needs to be investigated. The goal of $[87,91,92,88]$ is to show that some non-trivial rheological models can be recast into DIs with maximal monotone right-hand side as in Section 2.2. Other systems can be recast into FOSwP, like the system in Figure 4.4 (a). The dynamics of the massless spring is indeed given by:

$$
k\left(x-x_{e}(t)\right) \in-\mu m \operatorname{sgn}(\dot{x}) \Leftrightarrow \dot{x} \in-\mathcal{N}_{\left[-\frac{\mu m g}{k}+x_{e}(t), \frac{\mu m g}{k}+x_{e}(t)\right]}(x),
$$

where the equivalence uses the inversion of set-valued mappings, see Section A.2.4. The right-hand system in (4.16) is a FOSwP, while the left-hand system is a relay system, and is the simplest instance of a rate-independent system [269]. Controlled crawling robotic systems can be embedded in such processes [269, Sections 3,4$]$. The feedback control problem for crawlers, which has engineering interest [98], remains largely open and could certainly benefit from the studies on the control of FOSwP. When masses are non zero, they belong to the class of so-called "juggling systems" whose structure is particular $[140,131]$. It is noteworthy that so-called play operators of hysteresis (as in Figure 2.3 (b)), have the same FOSwP dynamics [71, Theorem 2.1] [557, Chapter III]. Visco-elasto-plastic contact forces are modeled with maximal monotone DIs in [437], and in a similar way with DVIs in [527].

4.2.3. Set-valued Coulomb's friction and extensions. Coulomb's friction model lends itself to complementarity, VI and DI formalisms [440, 149, 85], and naturally yields Lur'e set-valued systems [20,561]. Two-dimensional Coulomb's friction is represented in the tangent direction, by the mapping $v_{\mathrm{t}} \mapsto F_{\mathrm{t}} \in-\mu\left|F_{\mathrm{n}}\right| \operatorname{sgn}\left(v_{\mathrm{t}}\right)$. 
The dynamics can be interpreted when $F_{\mathrm{n}}$ is constant, through Filippov's DI, maximal monotone DI-see Section 3.4-, LCS-see Sections 3.10 and 3.11. It easily extends to include Stribeck (nonlinear or PWL) effects in Figure 4.4 (b). Such relations are close to some diode alternating current (DIAC) and silicon controller rectifier (SCR) set-valued models of electronics [14, Section 5.3.3], as well as tunnel diodes.

The main issue is that Coulomb's friction is not an associated law if $F_{\mathrm{n}}$ is not constant, i.e., it cannot be written as $\mathbb{R}^{3} \ni F_{i} \in \partial \varphi\left(v_{i}\right), v_{i} \in \mathbb{R}^{3}$, at a contact point $i$, in the local kinematics frame $[4,130]$, with $\varphi(\cdot)$ convex LSC. To clarify, let us consider the one-degree-of-freedom dynamics: $m \ddot{q}=F=\left(F_{\mathrm{n}}, F_{\mathrm{t}}\right)^{T}, 0 \leq q \perp F_{\mathrm{n}} \geq 0$ for the normal direction, and $F_{\mathrm{t}} \in-\mu\left|F_{\mathrm{n}}\right| \operatorname{sgn}\left(\dot{q}_{\mathrm{t}}\right)$ in the tangent direction. One can rewrite equivalently (using (3.12)): $F_{\mathrm{t}}=-\mu\left|F_{\mathrm{n}}\right|\left(1-2 \lambda_{\mathrm{t}}^{a}\right), 0 \leq\left(\begin{array}{c}\lambda_{\mathrm{t}}^{a} \\ \lambda_{\mathrm{t}}^{b}\end{array}\right) \perp\left(\begin{array}{c}\dot{q}_{\mathrm{t}}+\lambda_{\mathrm{t}}^{b} \\ 1-\lambda_{\mathrm{t}}^{a}\end{array}\right) \geq$ 0 . Thus we end up with an LCP with unknowns $F_{\mathrm{n}}, \lambda_{\mathrm{t}}^{a}, \lambda_{\mathrm{t}}^{b}$. The dynamics is however an NLCS (2.24) due to the product $\left|F_{\mathrm{n}}\right|\left(1-2 \lambda_{\mathrm{t}}^{a}\right)$ : this explains the complexity of systems with Coulomb's friction. In dimension three, the friction cone may be facetized $[4,521]$, yielding again an LCP. However this may not be desirable [4, Section 13.3.7]. One can write a cone complementarity problem (CCP) that keeps the friction cone intact, if De Saxcé's formulation with modified normal velocity $\hat{v}_{\mathrm{n}} \triangleq v_{\mathrm{n}}+\mu\left|v_{\mathrm{t}}\right|$ is used [219] [4, Section 3.9.2] [130, Section 5.3.3]. If $\mathcal{C}$ is the friction cone, then $\mathcal{C}^{\star} \in \hat{v} \perp F \in \mathcal{C}$ holds, the contact model is called a second order CCP [3], and the dynamics is a kind of complex NLCCS [4, Section 3.10] [130, Chapter 5] [385, Chapter 5]. Another approach using normal cones in an asymptotically singular metric, allows to recover an associated law "in the limit" when a small parameter vanishes [351, Section 4.1]. As alluded to above, extensions of Coulomb's friction, that remain in a set-valued setting and incorporate various nonlinear effects (Stribeck, Contensou etc), are possible [4, Section 3.9.4] [382]. Finally FOSwP of the form: $\dot{x} \in-\mathcal{N}_{\mathcal{S}(t)}(A \dot{x}+B x)$, find applications in quasistatic frictional contact problems (one neglects acceleration), involving materials with short memory [23].

4.3. Other Applications. Let us summarize various applications of the above mathematical formalisms, other than circuits or Lagrangian systems modelling.

- Plasticity, elastoplasticity: these were the first motivations for the FOSwP [437], stemming from a VI formulation of elastoplasticity [438, 369, 371, 527], and the ZOSwP in [27].

- Robust sliding-mode control (SMC): in first-order SMC, the closed-loop system has the dynamics: $\dot{x}=f(t, x)+w, w \in-a \operatorname{Sgn}(x)$, where $\operatorname{Sgn}(x)=\partial\left(\left|x_{1}\right|+\cdots+\right.$ $\left.\left|x_{n}\right|\right)$. The signum function can be replaced by a maximal monotone operator satisfying certain properties [17, 422, 421, 423]. The twisting controller (secondorder SMC) [454] yields a closed-loop system $\dot{x}=y, \dot{y} \in-a \operatorname{sgn}(x)-b \operatorname{sgn}(y)$ : it does not belong to maximal monotone DIs (2.16), it can be analysed with Filippov's DIs.

- Biological systems: DVIs [483], complementarity dynamical systems [7], Filippov's and Aizerman-Pyatnitskii's DIs [404, 405, 406], maximal monotone DIs with BV solutions [279], PWL systems [165, 216, 185, 164].

- Vaccination strategies: local PDS in $(2.31)[194,193]$.

- Neural networks: PDS (2.31) (c) [253], Filippov's DI [255], DIs as (2.16) with $\mathcal{F}(\cdot)=\partial \varphi(\cdot), \varphi(\cdot)$ non convex regular $[254]$, or with $\mathcal{F}(x)=\mathcal{N}_{\mathcal{S}}(x), \mathcal{S}$ constant convex closed set [218].

- Microscopic crowd dynamics: FOSwP with prox-regular $\mathcal{S}(t)[413,162]$, Lagrangian systems with impacts within Frémond's approach (see [130, Section 
4.3.4]) [471, 472], FOSwP with integral perturbation for sticky particle dynamics [119].

- Traffic flow networks, transportation systems: complementarity dynamical systems [77, 79, 78, 402, 460, 563, 231, 588, 587, 589], PDS in $(2.31)$ [213, 214, 212, $285,563,231,196]$, global PDS in $(2.32)$ [258].

- Networks with unilateral interactions: min-max systems which are LCS, see Section 3.11, [408].

- Economical systems and finance: complementarity systems [502], global and local PDS [258, 259, 228, 447, 211, 212, 310, 311, 251, 183, 328, 285].

- Human migration: PDS (double-layer dynamics) [448].

- Energy systems: complementarity systems (modelling in energy sector, large-scale systems) [260], global PDS (optimal energy management) [586].

- Queuing models: complementarity and maximal monotone mappings [159].

- Quantized feedback systems: filling-in the jumps in discontinuous quantized signals, using Krasovskii solutions (a definition similar to Filippov's convexification) $[180,181,333,334]$.

- Feedback control: control input calculated from a VI for viability in convex polytopes [524, Section 6.1], stabilization and regulation with state or output feedback [151, 524, 423], dynamic feedback calculated from a complementarity problem [337], quantized nonlinear control that yields sector bounded set-valued maps after filling-in the signals's jumps [334, 336, 393], optimal control of FOSwP [200, 201, 202, 207, 8, 237, 505, 506, 316, 160, 162, 161], optimal control of maximal monotone DIs [124], and LCS [555], controllability of relative degree zero LCS and of a class of PWL systems [150, 533], of planar evolution VIs [127], of juggling mechanical systems (SOSwP) [140], and of FOSwP [122], tracking control for SOSwP [142, 141, 118, 429, 428], robust sliding-mode set-valued trajectory tracking control of SOSwP $[17,421]$, robust sliding-mode set-valued control of linear invariant systems [422] (both with external disturbances and parameter uncertainties) using Moreau-Yosida and maximal monotone operators.

- Optimal control with constraints: pure state unilateral constraints yield LCS with higher relative degree that can be embedded into the HOSwP [128], mixed statecontrol unilateral constraints yield differential algebraic VIs [297].

- State observers, synchronization: maximal monotone DIs and FOSwP [138, 135, 215, 187, 230, 319, 511, 561], non convex FOSwP with prox-regular sets [33, 522], DI in normal cone to prox-regular set [31], LCS [306], mechanical systems with unilateral contact and impacts $[523,95,94]$, vibro-impact systems (see a definition in [130, Section 1.3.2]) [252, 262, 411, 417, 419, 418].

- Steepest descent algorithms and constrained optimization through dynamics: (the goal is to formulate a DI whose equilibrium is asymptotically stable and matches with the solution of an optimization problem, a Nash equilibrium, a Pareto minimum, etc) maximal monotone DIs $[56,58,60,57,49,48,72,223,227$, $275,412,477]$, autonomous [59, 222, 583], switching [276] and non-autonomous maximal monotone and subgradient DIs [55], global PDS in $(2.32)$ [53, 106, 111, 112, 400, 578], local PDS in (2.31) [62, Chapter 5, Section 6, Theorem $3][178,261,303,475,476,585,255]$, see also [189, 190, 244, 268, 517] who use another type of DI related to PWL systems and NLCS (see the end of Section 3.11), and [579] for the application of primal-dual gradient algorithm in model predictive control. It is also worth noting that a quite similar problem is solved in [542, Chapter 15] [355, 358, 356, 357, 246, 247, 580] and [270] (building on pi- 
oneer results in [493]), relying on sliding-mode control techniques to solve convex programming problems.

- Aerosols dynamics, atmospheric chemistry: MLCS and MLNCS of optimizationconstrained differential equations as in $(2.26)$ [148, 373, 51].

- Processes with phase changes: use of complementarity systems for thermodynamic equations [496, 342, 516], MPEC problems for optimal control of nonsmooth DAEs [484, 96], DIs with maximal monotone right-hand sides [198].

5. Well-posedness results. We now address broadly the question of existence and uniqueness of solutions for the mathematical models studied in Section 2. Giving a thorough treatment of all the works that exist for these system classes would involve too many details to be covered within this article. Rather, we review some of the fundamental techniques and provide a pedagogical summary of the methodology that goes in proving most of these results. Broadly speaking, we are interested in studying solutions which exist over a given interval of time (possibly large) such that the corresponding set of differential equations/inclusions and algebraic relations hold in appropriate sense. The fundamental motivation in addressing this set of results comes from the fact that the existence of solutions for systems modeled as sweeping processes, complementarity systems, evolution variational inequalities or other system classes treated in this article, cannot be obtained from the classical results in the literature on differential inclusions. For instance, the framework in [62, Chapter 2] is applicable to domains with nonempty interior and local (in time) solutions. Some results also appear if the mapping associated with the selection of the least norm element from the set-valued mapping satisfies some hypothesis. This concept of slow solutions obtained by selecting $\dot{x}(t)$ of minimal norm, has been used in Proposition 3.2 and Theorem 3.3 (with the constraint that solutions have to remain within the domain), see Example 7. But this concept of least-norm selection does not apply to FOSwP in general, and Example 4 shows that the unique solution of a FOSwP is not obtained by selecting the least norm element in the set-valued mapping. This makes the aforementioned results inapplicable to our systems. We note also that [220, Theorem 5.2] could at best guarantee the local existence of $\mathrm{AC}$ solutions for initial data in $\operatorname{int}(\mathcal{S}(t))$, under a linear growth condition. Conditions based on LSC or Lipschitz continuity for set-valued maps [513] are too restrictive for our purposes, and do not apply to our systems.

EXAMPLE 7. Consider the FOSwP in (3.6), that is under some assumptions equivalent to the LCS in (2.22). Then $\lambda(t)$ is the solution of the $L C P\left(q_{1}(t), M_{1}\right)$, employing notations of Section 2.4.1. Under the stated assumptions, $M_{1}=C B=$ $(C B)^{T} \succ 0$. Hence either $\xi \in \operatorname{int}(\Phi(u(t)))$ and $\lambda(t)=0$, or $\xi \in \operatorname{bd}(\Phi(u(t)))$ and $\lambda(t)$ solves an optimization problem, see Section $B$. This quadratic program computes an element of $\mathcal{N}_{\Phi(u(t))}(\xi)$ which guarantees that $\xi(t) \in \Phi(u(t))$ for all $t$, and that the right-hand side of (2.22a) is minimized. Since both the LCS and the FOSwP have the same solution, we infer that the solution of (3.6) is a kind of slow solution. This is similar to the case of Example 2, however with a relative degree $r=1$ instead of $r=0$.

For the solution concept under consideration (see for instance Definition 5.4), there are two most commonly used techniques for proving existence of solutions for the class of nonsmooth systems treated in this article. The first technique is based on introducing a single-valued regularization of the nonsmooth vector field in the dynamics with a parameter, and studying the convergence of regularized solutions with respect to that parameter. The other commonly employed method uses a dis- 
cretization of the system trajectories where a sequence of approximate solutions is constructed by varying the sampling time and the convergence is studied with respect to the discretization parameter. In both these techniques, the following sequence of steps is involved:

- Construction of a sequence of approximate solutions, either by regularization or interpolation of discretized solutions.

- Obtain uniform estimates on the maximum pointwise value, and the derivative or variation of the elements of the constructed sequence.

- Extracting a converging subsequence, and consider the limit of that sequence as a candidate solution.

- Show that the candidate solution satisfies the desired differential inclusion.

In case, if a candidate solution does not satisfy the differential inclusion under consideration, then it is only a weak solution.

We will demonstrate this sequence of steps in detail for the first order sweeping processes. The objective is to be able to recall different approaches and technicalities that go into proving several results that have appeared over the years. For the other classes of systems, we just present the main results, and give references to where the details of the foregoing steps can be found in the literature.

5.1. Regularization of FOSwP. We study the system described by:

$$
\dot{z}(t) \in-\mathcal{N}_{\mathcal{S}(t)}(z(t)), \quad z(0) \in \mathcal{S}(0),
$$

over an interval $[0, T]$ for some given $T>0$, with $\mathcal{S}:[0, T] \rightrightarrows \mathbb{R}^{n}$ a convex-valued mapping, and $z(t) \in \mathbb{R}^{n}$ being the state of the system. Equation (5.1) describes the simplest class of FOSwP, and we find it convenient to start our discussion on existence of solutions with this simple set-valued system where the right-hand side is a nonsmooth function of the state. For this system, we will prove the existence of solutions by using Moreau-Yosida regularization. Later, we consider a more general class of FOSwP and we provide a proof based on time-discretization. To prove the existence of solutions by regularization, we introduce the sequence of approximate solutions, the so-called Moreau-Yosida approximants $\left\{z_{\lambda}\right\}_{\lambda>0}$, which are obtained by solving the ODEs parameterized by $\lambda$ :

$$
\dot{z}_{\lambda}=-\frac{1}{\lambda}\left(z_{\lambda}(t)-\operatorname{proj}\left(z_{\lambda}(t), \mathcal{S}(t)\right)\right), \quad z_{\lambda}(0)=z_{0} \in \mathcal{S}(0) .
$$

We observe that, for each $\lambda>0$, the right-hand side of (5.2) is (globally) Lipschitz continuous, and hence there exists a continuously differentiable $z_{\lambda}:[0, T] \rightarrow \mathbb{R}^{n}$ such that (5.2) holds for every $t \geq 0$. The relation between the approximants $\left\{z_{\lambda}\right\}_{\lambda>0}$, and the solution of the inclusion (5.1) is described in Theorem 5.1 under the assumption that

(A1) The mapping $\mathcal{S}:[0, T] \rightrightarrows \mathbb{R}^{n}$ is closed and convex-valued for each $t \geq 0$, $\mathcal{S}(\cdot)$ varies in a Lipschitz continuous manner with time, that is, there exists a constant $L_{\mathcal{S}} \geq 0$, such that

$$
d_{\text {Haus }}\left(\mathcal{S}\left(t_{1}\right), \mathcal{S}\left(t_{2}\right)\right) \leq L_{\mathcal{S}}\left|t_{1}-t_{2}\right|, \forall t_{1}, t_{2} \geq 0,
$$

where $d_{\text {Haus }}$ denotes the Hausdorff distance between sets.

ThEOREM 5.1. Consider the FOSwP described by (5.1) and the sequence of solutions $\left\{z_{\lambda}\right\}_{\lambda>0}$ obtained from (5.2) on an interval $[0, T]$, and assume that (A1) holds; then 
- as $\lambda \rightarrow 0$, the sequence $z_{\lambda}(\cdot)$ converges uniformly to a Lipschitz continuous function $z(\cdot)$, and $z(\cdot)$ is a unique solution to the FOSwP (5.1),

- as $\lambda \rightarrow 0$, $\operatorname{proj}\left(z_{\lambda}(t), \mathcal{S}(t)\right)$ converges uniformly to $z(t)$ on $[0, T]$.

As stated at the beginning of the section, several key steps are involved in proving this result. For pedagogical exposition, we carry out these steps for this simple case in the sequel. The presentation in Sections 5.1, 5.2 and 5.4.1 is adapted from the material given in [238] and [427].

5.1.1. Estimates on the sequence $\left\{z_{\lambda}\right\}_{\lambda>0}$. For getting estimates on the norm of $z_{\lambda}(\cdot)$ and $\dot{z}_{\lambda}(\cdot)$, let us introduce the function $d_{\lambda}(t) \triangleq \inf _{y \in \mathcal{S}(t)}\left|y-z_{\lambda}(t)\right|$, so that $d_{\lambda}(t)=d_{\mathcal{S}(t)}\left(z_{\lambda}(t)\right)$. It is seen that $d_{\lambda}(t)=\left|z_{\lambda}(t)-\operatorname{proj}\left(z_{\lambda}(t), \mathcal{S}(t)\right)\right|$. To obtain a bound on $d_{\lambda}(t)$, we compute the derivative of $d_{\lambda}^{2}(t)$ :

$$
\frac{d}{d t} d_{\lambda}^{2}(t)=\lim _{\varepsilon \rightarrow 0} \frac{d_{\mathcal{S}(t+\varepsilon)}^{2}\left(z_{\lambda}(t+\varepsilon)\right)-d_{\mathcal{S}(t)}^{2}\left(z_{\lambda}(t+\varepsilon)\right)}{\varepsilon}+\frac{d_{\mathcal{S}(t)}^{2}\left(z_{\lambda}(t+\varepsilon)\right)-d_{\mathcal{S}(t)}^{2}\left(z_{\lambda}(t)\right)}{\varepsilon} .
$$

For the first term, we use the fact that

$$
\begin{aligned}
& d_{\mathcal{S}(t+\varepsilon)}^{2}\left(z_{\lambda}(t+\varepsilon)\right)-d_{\mathcal{S}(t)}^{2}\left(z_{\lambda}(t+\varepsilon)\right) \\
\leq & d_{\text {Haus }}(\mathcal{S}(t+\varepsilon), \mathcal{S}(t))\left(d_{\mathcal{S}(t+\varepsilon)}\left(z_{\lambda}(t+\varepsilon)\right)+d_{\mathcal{S}}(t)\left(z_{\lambda}(t+\varepsilon)\right)\right) \\
\leq & |\varepsilon| L_{\mathcal{S}}\left(d_{\mathcal{S}(t+\varepsilon)}\left(z_{\lambda}(t+\varepsilon)\right)+d_{\mathcal{S}(t)}\left(z_{\lambda}(t+\varepsilon)\right)\right) .
\end{aligned}
$$

For the second term, we first observe that

$$
\begin{aligned}
& d_{\mathcal{S}(t)}^{2}\left(z_{\lambda}(t+\varepsilon)\right)-d_{\mathcal{S}(t)}^{2}\left(z_{\lambda}(t)\right)=d_{\mathcal{S}(t)}^{2}\left(z_{\lambda}(t)+\varepsilon \dot{z}_{\lambda}(t)\right)-d_{\mathcal{S}(t)}^{2}\left(z_{\lambda}(t)\right) \\
+ & \left(d_{\mathcal{S}(t)}\left(z_{\lambda}(t+\varepsilon)\right)-d_{\mathcal{S}(t)}\left(z_{\lambda}(t)+\varepsilon \dot{z}_{\lambda}(t)\right)\right)\left(d_{\mathcal{S}(t)}\left(z_{\lambda}(t+\varepsilon)\right)+d_{\mathcal{S}(t)}\left(z_{\lambda}(t)+\varepsilon \dot{z}_{\lambda}(t)\right)\right)
\end{aligned}
$$

Since $z_{\lambda}(\cdot)$ is differentiable, $z_{\lambda}(t+\varepsilon)=z_{\lambda}(t)+\varepsilon \dot{z}_{\lambda}+o(\varepsilon)$ and hence $d_{\mathcal{S}(t)}\left(z_{\lambda}(t+\varepsilon)\right)-$ $d_{\mathcal{S}(t)}\left(z_{\lambda}(t)+\varepsilon \dot{z}_{\lambda}(t)\right)=o(\varepsilon)$. This results in

$$
\begin{aligned}
\lim _{\varepsilon \rightarrow 0} \frac{1}{\varepsilon}\left[d_{\mathcal{S}(t)}^{2}\left(z_{\lambda}(t)+\varepsilon \dot{z}_{\lambda}(t)\right)-d_{\mathcal{S}(t)}^{2}\left(z_{\lambda}(t)\right)\right] & =\left\langle\nabla d_{\mathcal{S}(t)}^{2}\left(z_{\lambda}(t)\right), \dot{z}_{\lambda}(t)\right\rangle \\
& =\left\langle z_{\lambda}(t)-\operatorname{proj}\left(z_{\lambda}(t), \mathcal{S}(t)\right), \dot{z}_{\lambda}(t)\right\rangle .
\end{aligned}
$$

Substitution yields $\frac{d}{d t} d_{\lambda}^{2}(t)=2 d_{\lambda}(t) \dot{d}_{\lambda}(t) \leq-\frac{1}{\lambda} d_{\lambda}^{2}(t)+2 L_{\mathcal{S}} d_{\lambda}(t)$, or equivalently $\frac{d}{d t} d_{\lambda}(t) \leq-\frac{1}{2 \lambda} d_{\lambda}(t)+L_{\mathcal{S}}$. By integration, the foregoing inequality gives

$$
d_{\lambda}(t) \leq \mathrm{e}^{-t / \lambda} d_{\lambda}(0)+L_{\mathcal{S}} \int_{0}^{t} \mathrm{e}^{-(t-s) / \lambda} d s=\lambda\left(1-\mathrm{e}^{-t / \lambda}\right) L_{\mathcal{S}}
$$

where we used the fact that $d_{\lambda}(0)=\left|z_{0}-\operatorname{proj}\left(z_{0}, \mathcal{S}(0)\right)\right|=0$ since $z_{0} \in \mathcal{S}(0)$. It then follows that for each $\lambda>0, \dot{z}_{\lambda}(t) \leq\left(1-\mathrm{e}^{-t / \lambda}\right) L_{\mathcal{S}} \leq L_{\mathcal{S}}$. Over a compact interval $[0, T]$, one readily gets the estimate: $\int_{0}^{T}\left|\dot{z}_{\lambda}(s)\right| d s \leq T L_{\mathcal{S}}$, and for each $t \in[0, T]$, $\left|z_{\lambda}(t)\right| \leq\left|z_{0}\right|+T L_{\mathcal{S}}$.

5.1.2. Extracting a converging subsequence. In the light of estimates obtained in the previous section, there exists a subsequence such that $z_{\lambda}(\cdot)$ converges to $z(\cdot)$ over this subsequence. One can in fact show that the convergence is strong.

LEMma 5.2. There exists a Lipschitz continuous function $z(\cdot)$, and a subsequence $\left\{z_{\lambda_{i}}\right\}_{i \in \mathbb{N}}$ which converges uniformly to $z(\cdot)$ on $[0, T]$. Moreover, for each $t \geq 0$, $z(t) \in \mathcal{S}(t)$. 
The proof of Lemma 5.2 is a consequence of the Arzelà-Ascoli theorem since the sequence $\left\{z_{\lambda_{i}}\right\}_{i \in \mathbb{N}}$ is continuously differentiable and $\left\{\dot{z}_{\lambda_{i}}\right\}_{i \in \mathbb{N}}$ is uniformly bounded by the constant $L_{\mathcal{S}}$. The limit function $z(\cdot)$ is also Lipschitz continuous with modulus $L_{\mathcal{S}}$. To see that $z(t) \in \mathcal{S}(t)$, we observe from (5.2) that a uniform bound on $\dot{z}_{\lambda}(\cdot)$ ensures that $\left|z_{\lambda}(t)-\operatorname{proj}\left(z_{\lambda}(t), \mathcal{S}(t)\right)\right| \rightarrow 0$ as $\lambda \rightarrow 0$. Consequently, $z_{\lambda}(t)$ gets closer to $\mathcal{S}(t)$ as $\lambda$ gets smaller, and since $\mathcal{S}(t)$ is closed, the limit $z(t)$ belongs to the set $\mathcal{S}(t)$.

5.1.3. Limit is a solution. The final piece in the proof of Theorem 5.1 is the following lemma, which states that the limit $z(\cdot)$ satisfies a VI.

LEMma 5.3. If $\varphi:[0, T] \rightarrow \mathbb{R}^{n}$ is a continuous selection of $\mathcal{S}(\cdot)$, that is, $\varphi(s) \in$ $\mathcal{S}(s)$, for each $s \in[0, T]$, then

$$
\int_{\left[t_{1}, t_{2}[\right.}\langle\varphi(s), \dot{z}(s)\rangle d s \geq \frac{1}{2}\left(\left\|z\left(t_{2}\right)\right\|^{2}-\left\|z\left(t_{1}\right)\right\|^{2}\right) .
$$

Proof. Let $\bar{z}_{\lambda}(s) \triangleq \operatorname{proj}\left(z_{\lambda}(s), \mathcal{S}(s)\right)$, then $s \mapsto \bar{z}_{\lambda}(s)$ is a continuous mapping. ${ }^{9}$ Since $\varphi(s) \in \mathcal{S}(s)$ and $\lambda$ is positive, it follows from the definition of the projections that

$$
\left\langle\varphi(s)-\bar{z}_{\lambda}(s), \dot{z}_{\lambda}(s)\right\rangle=\frac{1}{\lambda}\left\langle\varphi(s)-\bar{z}_{\lambda}(s), \bar{z}_{\lambda}(s)-z_{\lambda}(s)\right\rangle \geq 0,
$$

and hence $\int_{t_{1}}^{t_{2}}\left\langle\varphi(s), \dot{z}_{\lambda}(s)\right\rangle d s \geq \int_{t_{1}}^{t_{2}}\left\langle\bar{z}_{\lambda}(s), \dot{z}_{\lambda}(s)\right\rangle d s$. Since, at the points where $z_{\lambda}(\cdot)$ is differentiable, we have $\left\langle\bar{z}_{\lambda}(s), \dot{z}_{\lambda}(s)\right\rangle=\left\langle\bar{z}_{\lambda}(s)-z_{\lambda}(s), \dot{z}_{\lambda}(s)\right\rangle+\left\langle z_{\lambda}(s), \dot{z}_{\lambda}(s)\right\rangle=$ $\frac{1}{\lambda}\left|\bar{z}_{\lambda}(s)-z_{\lambda}(s)\right|^{2}+\left\langle z_{\lambda}(s), \dot{z}_{\lambda}(s)\right\rangle$, it follows that $\left\langle\bar{z}_{\lambda}(s), \dot{z}_{\lambda}(s)\right\rangle \geq\left\langle z_{\lambda}(s), \dot{z}_{\lambda}(s)\right\rangle$, and

$$
\int_{t_{1}}^{t_{2}}\left\langle\varphi(s), \dot{z}_{\lambda}(s)\right\rangle d s \geq \int_{t_{1}}^{t_{2}}\left\langle z_{\lambda}, \dot{z}_{\lambda}(s)\right\rangle d s=\frac{1}{2}\left(\left\|z_{\lambda}\left(t_{2}\right)\right\|^{2}-\left\|z_{\lambda}\left(t_{1}\right)\right\|^{2}\right) .
$$

We take limits with respect to $\lambda \rightarrow 0$. Since $z_{\lambda}(\cdot)$ converges pointwise to $z(\cdot)$, one has $\left\langle\varphi(s), \dot{z}_{\lambda}(s)\right\rangle \longrightarrow\langle\varphi(s), \dot{z}(s)\rangle$, for each $s \in\left[t_{1}, t_{2}\right]$, which yields (5.3).

We can now complete the proof of Theorem 5.1 using the aforementioned steps, and show that the limiting function $z(\cdot)$ is indeed a solution to (5.1). This is done by showing that for any $\zeta \in \mathcal{S}(t)$, it holds for almost every $t \geq 0$ that $\langle\zeta-z(t), \dot{z}(t)\rangle \geq 0$, which in turn implies $\dot{z}(t) \in-\mathcal{N}_{\mathcal{S}(t)}(z(t))$. This is indeed the case since for every $\zeta \in \mathcal{S}(t)$, we can choose a Lipschitz continuous function $\varphi:[t, T]$ such that, due to Lemma 5.3, we get

$$
\int_{[t, t+\varepsilon[}\langle\varphi(s), \dot{z}(s)\rangle d s \geq \frac{1}{2}\left(\|z(t+\varepsilon)\|^{2}-\|z(t)\|^{2}\right)
$$

and by letting $\varphi(s)=\zeta-(\zeta-\varphi(s))$, we have

$$
\langle\zeta, z(t+\varepsilon)-z(t)\rangle-\int_{t}^{t+\varepsilon}\langle\zeta-\varphi(s), \dot{z}(s)\rangle d s \geq \frac{1}{2}\langle z(t+\varepsilon)+z(t), z(t+\varepsilon)-z(t)\rangle
$$

\footnotetext{
${ }^{9}$ This is because, by construction, $z_{\lambda}(\cdot)$ is continuous, and for any $x \in \mathbb{R}^{n}$ one can show that $s \mapsto \operatorname{proj}(x, \mathcal{S}(s))$ is continuous if $\mathcal{S}(\cdot)$ is continuous in the Hausdorff sense. To see the later, let $s^{\prime}, s^{\prime \prime} \in[0, T]$, then $\left|x-\operatorname{proj}\left(x, \mathcal{S}\left(s^{\prime \prime}\right)\right)\right| \leq\left|x-\operatorname{proj}\left(\operatorname{proj}\left(x, \mathcal{S}\left(s^{\prime}\right)\right), \mathcal{S}\left(s^{\prime \prime}\right)\right)\right| \leq\left|x-\operatorname{proj}\left(x, \mathcal{S}\left(s^{\prime}\right)\right)\right|+$ $\left|\operatorname{proj}\left(x, \mathcal{S}\left(s^{\prime}\right)\right)-\operatorname{proj}\left(\operatorname{proj}\left(x, \mathcal{S}\left(s^{\prime}\right)\right), \mathcal{S}\left(s^{\prime \prime}\right)\right) \leq\right| x-\operatorname{proj}\left(x, \mathcal{S}\left(s^{\prime}\right)\right) \mid+d_{\text {Haus }}\left(S\left(s^{\prime}\right), S\left(s^{\prime \prime}\right)\right)$. In particular, using [427, Chap. 0, Prop. 4.7], we obtain $\left|\operatorname{proj}\left(x, \mathcal{S}\left(s^{\prime}\right)\right)-\operatorname{proj}\left(x, \mathcal{S}\left(s^{\prime}\right)\right)\right| \leq 4 d_{\text {Haus }}\left(S\left(s^{\prime}\right), S\left(s^{\prime \prime}\right)\right) \mid x-$ $\operatorname{proj}\left(x, \mathcal{S}\left(s^{\prime}\right)\right) \mid+2 d_{\text {Haus }}^{2}\left(S\left(s^{\prime}\right), S\left(s^{\prime \prime}\right)\right)$.
} 
or

$$
\left\langle\zeta-\frac{1}{2}(z(t+\varepsilon)+z(t)), z(t+\varepsilon)-z(t)\right\rangle \geq-\varepsilon \max _{s \in[t, t+\varepsilon[}|\zeta-\varphi(s)| .
$$

Since $z(\cdot)$ is Lipschitz continuous, $z(\cdot)$ is differentiable almost everywhere on $[0, T]$. Thus, for almost every $t \in[0, T]$, where $z(\cdot)$ is differentiable, dividing the last inequality by $\varepsilon$ and letting $\varepsilon$ tend to zero, we get, $\langle\zeta-z(t), \dot{z}(t)\rangle \geq 0$ for each $\zeta \in \mathcal{S}(t)$.

5.1.4. Adding Lipschitz perturbations. We now use the foregoing result to address the systems with perturbations of the form:

$$
\dot{z}(t) \in-\mathcal{N}_{\mathcal{S}(t)}(z(t))+\widetilde{f}(t), \quad z(0)=z_{0} \in \mathcal{S}(0)
$$

where $\tilde{f}:[0, T] \rightarrow \mathbb{R}^{n}$ is AC. This is a preliminary step in addressing more general state-dependent perturbations considered in (5.7). To define a solution for (5.4), we introduce the set-valued mapping $\widetilde{\mathcal{S}}(t)=\mathcal{S}(t)+\int_{0}^{t} \widetilde{f}(s) d s$, and let $z(t)=\widetilde{z}(t)-$ $\int_{0}^{t} \widetilde{f}(s) d s$, where $\dot{\widetilde{z}} \in-\mathcal{N}_{\widetilde{\mathcal{S}}}(\widetilde{z})$ with $\widetilde{z}(0)=z_{0}$. From the previous discussion, $z(\cdot)$ indeed is a solution to (5.4). Next, we use the above result to obtain a bound on $\left|z_{1}(t)-z_{2}(t)\right|$, for each $t \geq 0$, where $z_{i}, i=1,2$, satisfies

$$
\dot{z}_{i}(t) \in-\mathcal{N}_{\mathcal{S}(t)}\left(z_{i}\right)+\tilde{f}_{i}(t)
$$

for some AC functions $\tilde{f}_{i}:[0, T] \rightarrow \mathbb{R}^{n}$. To compute this bound, it is seen that the monotonicity of $\mathcal{N}_{\mathcal{S}}(\cdot)$ yields

$$
\begin{aligned}
\frac{1}{2} \frac{d}{d t}\left|z_{1}(t)-z_{2}(t)\right|^{2} & =\left\langle\dot{z}_{1}(t)-\dot{z}_{2}(t), z_{1}(t)-z_{2}(t)\right\rangle \leq\left\langle\tilde{f}_{1}(t)-\tilde{f}_{2}(t), z_{1}(t)-z_{2}(t)\right\rangle \\
& \leq\left|\tilde{f}_{1}(t)-\tilde{f}_{2}(t)\right| \cdot\left|z_{1}(t)-z_{2}(t)\right|
\end{aligned}
$$

where the first inequality is due to the monotonicity of $\mathcal{N}_{\mathcal{S}(t)}(\cdot)$, and the second inequality is due to Cauchy-Schwarz. Letting $Z(t) \triangleq\left|z_{1}(t)-z_{2}(t)\right|^{2}$, the above inequality is rewritten as: $\dot{Z}(t) \leq 2\left|\tilde{f}_{1}(t)-\tilde{f}_{2}(t)\right| \sqrt{Z(t)}$. Applying the comparison lemma for solution of ODEs [349, Lemma 3.4], we get

$$
\left|z_{1}(t)-z_{2}(t)\right| \leq\left|z_{1}(0)-z_{2}(0)\right|+\int_{0}^{t}\left|\tilde{f}_{1}(s)-\tilde{f}_{2}(s)\right| d s .
$$

We use this estimate to address the question of existence of solutions to

$$
\dot{z} \in-\mathcal{N}_{\mathcal{S}(t)}(z)+f(t, z), \quad z(0)=z_{0} \in \mathcal{S}(0) .
$$

(A2) There exists $\rho>0$ such that

$$
\left|f\left(t, x_{1}\right)-f\left(t, x_{2}\right)\right| \leq \rho\left|x_{1}-x_{2}\right|, \quad \forall x_{1}, x_{2} \in \mathbb{R}^{n} .
$$

We now make use of the estimate obtained in (5.6). Towards this end, consider a sequence of $\mathrm{AC}$ functions $\left\{z_{i}\right\}_{i \in \mathbb{N}}$, with $z_{1}(t)=z(0), t \in[0, T]$, and $z_{i+1}(\cdot)$, for $i \geq 1$ obtained as a solution to the inclusion

$$
\dot{z}_{i+1}(t) \in-\mathcal{N}_{\mathcal{S}(t)}\left(z_{i+1}(t)\right)+f\left(t, z_{i}(t)\right),
$$


with initial condition $z_{i}(0)=z(0), i \in \mathbb{N}$. Under the Lipschitz continuity assumption, it follows that for each compact interval $[0, T]$, there exists a constant $\rho$ such that

$$
\left|f\left(t, z_{i}(t)\right)-f\left(t, z_{i-1}(t)\right)\right| \leq \rho\left|z_{i}(s)-z_{i-1}(s)\right| .
$$

Then, from (5.6), we have, for each $t \in[0, T]:\left|z_{i+1}(t)-z_{i}(t)\right| \leq \int_{0}^{t} \rho\left|z_{i}(s)-z_{i-1}(s)\right| d s$. By induction this leads to $\left|z_{i+1}(t)-z_{i}(t)\right| \leq \frac{(\rho t)^{i}}{i !}\left\|z_{2}-z_{1}\right\|_{\mathcal{L}_{\infty}\left([0, T], \mathbb{R}^{n}\right)}$. Thus, the sequence $\left\{z_{i}\right\}_{i=1}^{\infty}$ converges uniformly on every compact interval [0,T], and it can be shown that $z(t) \triangleq \lim _{i \rightarrow \infty} z_{i}(t)$ is a solution to system (5.7). Uniqueness of solutions can be shown to hold easily for the inclusion (5.7) due to monotonicity of the operator $\mathcal{N}_{\mathcal{S}(t)}(\cdot)$ for each $t$, see also Section 5.2.4.

5.2. Discretization via Time-Stepping Algorithm. Thus far, we have considered solutions which are AC. This is primarily because the variation in the setvalued mapping $\mathcal{S}(\cdot)$ is bounded by an AC function. If one relaxes $\mathcal{S}(\cdot)$ to evolve such that the Hausdorff distance between the values of $\mathcal{S}(\cdot)$ at any two time instants is bounded by the variation of a $\mathrm{BV}$ function, then the resulting solution will be a BV function. In what follows, please refer to Appendix E for definitions, as we study rigorously the existence of solutions where the regularity of $\mathcal{S}(\cdot)$ with respect to time is relaxed as follows:

(A3) there exists a nondecreasing RCBV function $\mu_{\mathcal{S}}:[0, T] \rightarrow[0, \infty)$, such that its differential measure, denoted by $d \mu$, has the property that

$$
d_{\text {Haus }}\left(\mathcal{S}\left(t_{1}\right), \mathcal{S}\left(t_{2}\right)\right) \leq d \mu\left(\left(t_{1}, t_{2}\right]\right)=\mu_{\mathcal{S}}\left(t_{2}\right)-\mu_{\mathcal{S}}\left(t_{1}\right) \text {, for every } t_{2} \geq t_{1} \geq 0 .
$$

We also relax the regularity assumption on $f(\cdot, x)$ as follows:

(A4) There exists a Lebesgue integrable function $\beta:[0, T] \rightarrow[0, \infty)$ such that $|f(t, x)| \leq \beta(t)(1+|x|)$, for all $x \in \mathbb{R}^{n}$, for all $t \geq 0$.

When working with $\mathrm{BV}$ functions, the FOSwP (5.7) has to be interpreted in the sense of measures associated with the BV functions, and care must be taken in defining the appropriate solution concept.

DEFINITION 5.4. Consider the system (5.7) under the assumptions (A3) and (A4). An RCBV function $x:[0, T] \rightarrow \mathbb{R}^{n}$ is called a solution to system (5.7) if there exists a nonnegative Radon measure $d \nu$, which is $A C$ equivalent to $d t+d \mu$ such that the differential measure $d x$ is $A C$ with respect to $d \nu, \frac{d x}{d \nu} \in \mathcal{L}^{1}\left([0, T], \mathbb{R}^{n} ; d \nu\right)$, and the following relation holds:

$$
\frac{d x}{d \nu}(t) \in f(t, x) \frac{d t}{d \nu}(t)-\mathcal{N}_{\mathcal{S}(t)}\left(x\left(t^{+}\right)\right), \quad d \nu-\text { a.e. } t \in[0, T] .
$$

This concept of solution is borrowed from the literature on sweeping processes with BV solutions, see for example [238], and this solution concept is independent of the choice of the measure $d \nu$ (because $\mathcal{N}_{\mathcal{S}(t)}(x)$ is a cone for any $x \in \mathcal{S}(t)$ ). An important observation that can be made from (5.8) is that, at time $t_{i}$, if there is a jump in $\mu_{\mathcal{S}}$, so that the measure $d \mu$ is supported on the singleton $\left\{t_{i}\right\}$, then a jump in the state trajectory $x$ at $t_{i}$ is also possible, and this jump is represented by:

$$
x\left(t_{i}^{+}\right)-x\left(t_{i}^{-}\right) \in-\mathcal{N}_{\mathcal{S}\left(t_{i}\right)}\left(x\left(t_{i}^{+}\right)\right),
$$

which is equivalent by (A.6), to solving the following quadratic optimization problem over a convex set: $x\left(t_{i}^{+}\right)=\arg \min _{v \in \mathcal{S}\left(t_{i}\right)}\left\|x\left(t_{i}^{-}\right)-v\right\|^{2}$. With this solution concept we now have the following result: 


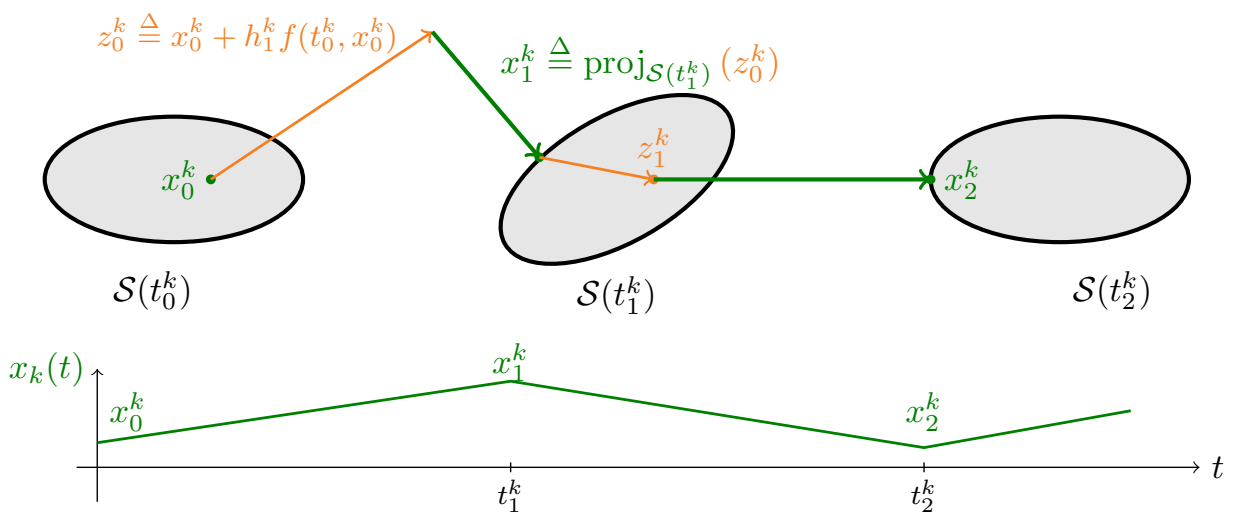

Fig. 5.1: Moreau's catching-up time-stepping algorithm for FOSwP.

TheOrem 5.5. Consider system (5.7) under the assumptions (A2), (A3) and (A4); then there exists a unique solution $x(\cdot)$ in the sense of Definition 5.4.

We will prove Theorem 5.5 by using Moreau's catching-up time-stepping algorithm, which relates to constructing a sequence of approximate solutions using discretization in time. In other words, we introduce a sequence of functions $\left\{x_{k}:[0, T] \rightarrow\right.$ $\left.\mathbb{R}^{n}\right\}_{k \in \mathbb{N}}$, and to construct the $k$-th element of this sequence, we consider a partition of the interval $[0, T]$ by choosing the time instants $0=t_{0}^{k}, t_{1}^{k}, t_{2}^{k}, \cdots, t_{N^{k}}^{k}=T$. The function $x_{k}(\cdot)$ is obtained by interpolating certain points $x_{i}^{k}$, over the interval $\left[t_{i}^{k}, t_{i+1}^{k}[\right.$, for $0 \leq i \leq N^{k}-1$, where we take $x_{0}^{k}(0)=x(0)$ for each $k \in \mathbb{N}$, and let

$$
x_{i+1}^{k} \triangleq \operatorname{proj}\left(x_{i}^{k}+h_{i+1}^{k} f\left(\hat{t}_{i}^{k}, x_{i}^{k}\right), \mathcal{S}\left(t_{i+1}^{k}\right)\right),
$$

where $\widehat{t}_{i}^{k} \in\left[t_{i}^{k}, t_{i+1}^{k}\left[\right.\right.$ is chosen such that $\beta\left(\hat{t}_{i}^{k}\right) \leq \inf _{s \in\left[t_{i}^{k}, t_{i+1}^{k}\right]} \beta(s)$, with $\beta(\cdot)$ given in Assumption (A4). We will use the notation $f_{i}^{k} \triangleq f\left(\widehat{t}_{i}^{k}, x_{i}^{k}\right)$, and for each $k \in \mathbb{N}$, $h_{i+1}^{k} \triangleq t_{i+1}^{k}-t_{i}^{k}$ represents the corresponding sampling size (which could be uniform with respect to $i$ ). The sequence is thus obtained by decreasing $h^{k} \triangleq \max _{1 \leq i \leq N^{k}} h_{i}^{k}$ with $k$ increasing, and letting $h^{k} \rightarrow 0$ as $k \rightarrow \infty$. To see why (5.9) provides a discretization of (5.7), note that

$$
\frac{x_{i+1}^{k}-x_{i}^{k}}{h_{i+1}^{k}}-f\left(t_{i+1}^{k}, x_{i}^{k}\right) \in-\mathcal{N}_{\mathcal{S}\left(t_{i+1}^{k}\right)}\left(x_{i+1}^{k}\right) .
$$

Next, to define $x_{k}$, we introduce the positive Radon measure $d \nu$ on the interval $[0, T]$, $d \nu \triangleq d \mu+d t$, and let

$$
x_{k}(t) \triangleq x_{i}^{k}+\frac{\left.\left.d \nu(] t_{i}^{k}, t\right]\right)}{\left.\left.d \nu(] t_{i}^{k}, t_{i+1}^{k}\right]\right)}\left(x_{i+1}^{k}-x_{i}^{k}-h_{i+1}^{k} f_{i}^{k}\right)+\left(t-t_{i}^{k}\right) f_{i}^{k} .
$$

Alternatively, by letting $f_{k}(s) \triangleq f_{i}^{k}$, for $s \in\left[t_{i}^{k}, t_{i+1}^{k}[\right.$, we can write

$$
x_{k}(t)=x_{k}(0)+\int_{] 0, t]} \Pi_{k}(s) d \nu(s)+\int_{] 0, t]} f_{k}(s) d t(s)
$$


where, we let $\Pi_{k}(t) \triangleq \sum_{j=0}^{N_{t}^{k}} \frac{x_{j+1}^{k}-x_{j}^{k}-h_{j+1}^{k} f_{j}^{k}}{\left.d \nu\left(\mid t_{j}^{k}, t_{j+1}^{k}\right]\right)} \mathbb{1}_{\left.] t_{j}^{k}, t_{j+1}^{k}\right]}(t)$, in which $N_{t}^{k} \triangleq \max \left\{i \mid t_{i+1}^{k} \leq\right.$ $t\}$, for $t \in[0, T]$. In the remainder of this section, it will be shown that the sequence in (5.10) indeed converges, as $k \rightarrow \infty$, to an RCBV function, which is a solution of system (5.7) in the sense of Definition 5.4.

5.2.1. Estimates on the sequence. The first step in establishing the existence of the limit is to obtain a uniform bound on the $\mathcal{L}^{\infty}$-norm of the sequence $\left\{x_{k}\right\}_{k \in \mathbb{N}}$.

LEMMA 5.6. The sequence $\left\{x_{k}\right\}_{k \in \mathbb{N}}$ is uniformly bounded, and in particular, there is a constant $C_{x_{0}, T}>0$ such that, for each $k \in \mathbb{N}$, and $t \in[0, T]$, the inequality $\left|x_{k}(t)\right| \leq C_{x_{0}, T}$ holds.

Proof. From the definition of $x_{i}^{k}$ in (5.9), it is seen that

$$
\left.\left.\left|x_{i+1}^{k}-x_{i}^{k}-h_{i+1}^{k} f_{i}^{k}\right|=d_{\mathcal{S}\left(t_{i+1}^{k}\right)}\left(x_{i}^{k}-h_{i+1}^{k} f_{i}^{k}\right) \leq d \mu(] t_{i}^{k}, t_{i+1}^{k}\right]\right)+h_{i+1}^{k}\left|f_{i}^{k}\right|,
$$

and this inequality results in $\left.\left.\left|x_{i+1}^{k}\right| \leq\left|x_{i}^{k}\right|+d \mu(] t_{i}^{k}, t_{i+1}^{k}\right]\right)+2 h_{i+1}^{k}\left|f_{i}^{k}\right|$. By using the bound on the vector field $f(\cdot)$ in (A4), we get

$$
\left.\left.\left|x_{i+1}^{k}\right| \leq\left|x_{i}^{k}\right|+d \mu(] t_{i}^{k}, t_{i+1}^{k}\right]\right)+2 h_{i+1}^{k} \beta\left(\hat{t}_{i}^{k}\right)\left(1+\left|x_{i}^{k}\right|\right) .
$$

A recursive application of the foregoing estimate leads to

$$
\left.\left.\left|x_{i+1}^{k}\right| \leq\left|x_{0}^{k}\right|+\sum_{j=0}^{i} d \mu(] t_{j}^{k}, t_{j+1}^{k}\right]\right)+2 h_{j+1}^{k} \beta\left(\hat{t}_{j}^{k}\right)\left(1+\left|x_{j}^{k}\right|\right),
$$

which by choosing $x_{0}^{k}=x_{0}$, and letting $C_{\beta} \triangleq \int_{[0, T]} \beta(s) d s$, results in

$$
\left|x_{i+1}^{k}\right| \leq\left|x_{0}\right|+d \mu([0, T])+2 C_{\beta}+2 \sum_{j=0}^{i} h_{j+1}^{k} \beta\left(\hat{t}_{j}^{k}\right)\left|x_{j}^{k}\right| .
$$

Using the discrete version of Gronwall-Bellman Lemma given in Appendix E.2, and the integrability of $\beta(\cdot)$, we obtain a bound on $x_{i+1}^{k}$ independent of $i$ and $k$, so that $\left|x_{i+1}^{k}\right| \leq \bar{C}_{\max }$, where $\bar{C}_{\max } \triangleq\left(\left|x_{0}\right|+d \mu([0, T])+2 C_{\beta}\right)\left(1+\mathrm{e}^{C_{\beta}} C_{\beta}\right)$. This bound can now be used to get a uniform bound on $\left\{x_{k}(\cdot)\right\}_{k \in \mathbb{N}}$. To do so, we first observe that

$$
h_{i+1}^{k}\left|f_{i}^{k}\right| \leq h_{i+1}^{k} \beta\left(\hat{t}_{i}^{k}\right)\left(1+\left|x_{i}^{k}\right|\right) \leq\left(1+\bar{C}_{\max }\right) \int_{t_{i}}^{t_{i+1}} \beta(s) d s .
$$

Substituting this last expression in (5.10) and using (5.11), we can get a constant $C_{x_{0}, T}$ such that $\left|x_{k}(t)\right| \leq C_{x_{0}, T}$, for all $t \in[0, T]$.

Next step in establishing the existence of a limit, is to compute a bound on the variation of each element of the sequence $\left\{x_{k}\right\}_{k \in \mathbb{N}}$.

LEMma 5.7. The total variation of each element of the sequence $\left\{x_{k}\right\}_{k \in \mathbb{N}}$ is uniformly bounded, that is, there exists $C_{\text {var }}$ such that for each $k \in \mathbb{N}$, we have

$$
\int_{[0, t]}\left\|\frac{d x_{k}}{d \nu}(s)\right\| d \nu(s) \leq C_{v a r} d \nu([0, t])
$$

for $d \nu$-almost every $t \in[0, t]$. 
Proof. It is seen from (5.10) that $x_{k}(t)=x_{k}(0)+\int_{[0, t]}\left[\Pi_{k}(s)-f_{k}(s) \frac{d t}{d \nu}(s)\right] d \nu(s)$, which results in

$$
\frac{d x_{k}}{d \nu}(t)+f_{k}(t) \frac{d t}{d \nu}(t)=\Pi_{k}(t)=\sum_{j=0}^{N_{t}^{k}} \frac{x_{j+1}^{k}-x_{j}^{k}-h_{j+1}^{k} f_{j}^{k}}{\left.\left.d \nu(] t_{j}^{k}, t_{j+1}^{k}\right]\right)} \mathbb{1}_{] t_{j}^{k}, t_{j+1}^{k}\right]}(t) .
$$

From there, it can be shown that

$$
\begin{aligned}
\left\|\frac{d x_{k}}{d \nu}(t)\right\| & \leq\left|f_{k}(t)\right|+\max _{0 \leq j \leq N_{t}^{k}}\left|x_{j+1}^{k}-x_{j}^{k}-h_{j+1}^{k} f_{j}^{k}\right|, \\
& \leq \max _{0 \leq j \leq N_{t}^{k}}\left|x_{j+1}^{k}-x_{j}^{k}-h_{j+1}^{k} f_{j}^{k}\right|+\left|f_{j}^{k}\right| .
\end{aligned}
$$

Because of the uniform bounds on $\left\{x_{k}(\cdot)\right\}$ and $\left\{f_{k}(\cdot)\right\}$, for each $k$, the right-hand side can be bounded by a constant $C_{v a r}$, and hence the total variation of $x_{k}$ is uniformly bounded by $C_{\text {var }} d \nu([0, T])$ for each $k \in \mathbb{N}$.

5.2.2. Extracting the converging subsequence. Because of Lemma 5.6 and Lemma 5.7 , possibly by extracting a subsequence, it is seen that $\frac{d x_{k}}{d \nu}$ converges weakly in the functional space $\mathcal{L}^{2}\left([0, T], \mathbb{R}^{n} ; d \nu\right)$ to some map $g(\cdot)$. So, for any $t \in[0, T]$,

$$
\int_{[0, t[} \frac{d x_{k}}{d \nu}(s) d \nu(s) \stackrel{\text { weakly in } \mathbb{R}^{n}}{\longrightarrow} \int_{[0, t[} g(s) d \nu(s)
$$

Also, due to strong convergence of $x_{k}(t)$ to $x(t)$ in $\mathbb{R}^{n}$ for each $t \in[0, T]$, we have $x(t)=x_{0}+\int_{[0, t[} g(s) d \nu(s)$, and hence $x(\cdot)$ is a right-continuous function of bounded variation, and the measure $d x$ has a density $g=\frac{d x}{d \nu} \in \mathcal{L}^{2}\left([0, T], \mathbb{R}^{n} ; d \nu\right)$ relatively to $d \nu$. As a result, $\frac{d x_{k}}{d \nu} \frac{\text { weakly in }}{\mathcal{L}^{2}\left([0, T], \mathbb{R}^{n} ; d \nu\right)} \frac{d x}{d \nu}$, and this leads to $\frac{d x_{k}}{d \nu}(\cdot) \frac{\text { weakly in }}{\mathcal{L}^{1}\left([0, T], \mathbb{R}^{n} ; d \nu\right)} \frac{d x}{d \nu}(\cdot)$. We now show that the sequence $f_{k}(\cdot)$ converges. It is seen that $f_{k}(\cdot)$ is weakly relatively compact in $\mathcal{L}^{2}\left([0, T], \mathbb{R}^{n} ; d \nu\right)$. In particular, because of the bound on $f_{k}(\cdot)$, $f_{k}(s)=f\left(\hat{t}_{i}^{k}, x_{i}^{k}\right), s \in\left[t_{i}^{k}, t_{i+1}^{k}\right]$, converges to $f\left(s, x_{k}(s)\right)$ weakly in $\mathcal{L}^{1}\left([0, T], \mathbb{R}^{n} ; d t\right)$, and consequently

$$
f_{k}(\cdot) \frac{d t}{d \nu}(\cdot) \underset{\mathcal{L}^{1}\left([0, T], \mathbb{R}^{n} ; d \nu\right)}{\stackrel{\text { weakly in }}{\longrightarrow}} f(\cdot, x(\cdot)) \frac{d t}{d \nu}(\cdot) .
$$

5.2.3. Limit is a solution. The last part of the proof is to show that $x(\cdot)$ obtained as the limit of a converging subsequence, is a solution to the differential inclusion. Invoking Mazur's Lemma in Section E.1, there exists a sequence $\zeta_{n}(\cdot)$, such that,

$$
\zeta_{n}(\cdot) \in \operatorname{co}\left\{\frac{d x_{k}}{d \nu}(\cdot)-f_{k}(\cdot) \frac{d t}{d \nu}(\cdot): k \geq n\right\}
$$

and $\zeta_{n}$ converges strongly in $\mathcal{L}^{1}\left([0, T], \mathbb{R}^{n} ; d \nu\right)$, i.e.,

$$
\zeta_{n}(t) \longrightarrow \frac{d x}{d \nu}(t)-f(t, x(t)) \frac{d t}{d \nu}(t), \quad d \nu \text {-a.e. } t \in[0, T] .
$$

Next, we recall from our construction that, for each $t \in\left[t_{i}^{k}, t_{i+1}^{k}\right]$,

$$
\frac{d x_{k}}{d \nu}(t)-f_{k}(t) \frac{d t}{d \nu}(t)=\Pi_{k}(t) \in-\mathcal{N}_{\mathcal{S}\left(t_{i+1}^{k}\right)}\left(x\left(t_{i+1}^{k}\right)\right),
$$


and in particular the left-hand side is uniformly bounded, for each $k \in \mathbb{N}, t \in[0, T]$. Next, for each $t \in[0, T]$, it is possible to construct a sequence, $t_{i_{k}+1}^{k} \rightarrow t$ such that $t \in\left[t_{i_{k}}^{k}, t_{i_{k}+1}^{k}\right]$ and $x_{k}\left(t_{i_{k}+1}^{k}\right) \rightarrow x(t)$. For each $\xi \in \mathcal{S}(t)$, there also exists a sequence $\xi_{k} \in \mathcal{S}\left(t_{i_{k}+1}^{k}\right)$ such that $\xi_{k} \rightarrow \xi \in \mathcal{S}(t)$, and we thus have

$$
\begin{aligned}
\left\langle\frac{d x_{k}}{d \nu}(t)-f_{k}(t) \frac{d t}{d \nu}(t), x(t)\right. & -\xi\rangle=\left\langle\frac{d x_{k}}{d \nu}(t)-f_{k}(t) \frac{d t}{d \nu}(t), x_{k}\left(t_{i_{k}+1}^{k}\right)-\xi_{k}\right\rangle \\
& +\left\langle\frac{d x_{k}}{d \nu}(t)-f_{k}(t) \frac{d t}{d \nu}(t), x(t)-x_{k}\left(t_{i_{k}+1}^{k}\right)+\xi_{k}-\xi\right\rangle .
\end{aligned}
$$

The first term on the right-hand side is non-positive and from the bounds computed in Lemma 5.6 and Lemma 5.7, and using (A4), we thus get, for some $C>0$,

$$
\left\langle\frac{d x_{k}}{d \nu}(t)-f_{k}(t) \frac{d t}{d \nu}(t), x(t)-\xi\right\rangle \leq C\left(\left|x(t)-x_{k}\left(t_{i_{k}+1}^{k}\right)\right|+\left|\xi_{k}-\xi\right|\right) .
$$

Hence, for every $\xi \in \mathcal{S}(t), \lim _{k \rightarrow \infty}\left\langle\frac{d x_{k}}{d \nu}(t)-f_{k}(t) \frac{d t}{d \nu}(t), x(t)-\xi\right\rangle \leq 0$, and

$$
\left\langle\frac{d x}{d \nu}(t)-f(t, x(t)) \frac{d t}{d \nu}(t), x(t)-\xi\right\rangle \leq \limsup _{n \rightarrow \infty}\left\langle\zeta_{n}, x(t)-\xi\right\rangle \leq 0,
$$

which is equivalent to: $\frac{d x}{d \nu}(t)-f(t, x(t)) \frac{d t}{d \nu}(t) \in-\mathcal{N}_{\mathcal{S}(t)}(x(t)), \quad d \nu$ - a.e. $t \in[0, T]$, and we have the desired inclusion.

5.2.4. Forward uniqueness and continuous dependence on initial data. One of the nicest properties of the systems governed by FOSwP, is that one can invoke maximal monotonicity of the normal cone operator, to prove the forward uniqueness of solutions and continuous dependence of the solution on the initial condition and system data. This property does not carry over to SOSwP and HOSwP as we will see later. More precisely, if $x_{j}:[0, T] \rightarrow \mathbb{R}^{n}, j=1,2$, are two solutions satisfying the DI (5.8) with initial conditions $x_{j}(0)=x_{0}^{j}$, then (using Assumption (A2) and the maximal monotonicity of the normal cone mapping)

$$
\begin{aligned}
\frac{1}{2} \frac{d}{d \nu}\left|x_{1}(t)-x_{2}(t)\right|^{2} & =\left\langle\frac{d x_{1}}{d \nu}(t)-\frac{d x_{2}}{d \nu}(t), x_{1}(t)-x_{2}(t)\right\rangle \\
& \leq\left\langle f\left(t, x_{1}(t)\right)-f\left(t, x_{2}(t)\right), x_{1}(t)-x_{2}(t)\right\rangle \frac{d t}{d \nu}(t) \\
& \leq \rho\left|x_{1}(t)-x_{2}(t)\right|^{2} \frac{d t}{d \nu}(t) .
\end{aligned}
$$

Integrating both sides with respect to $d \nu$, we get, for each $t \in[0, T]$,

$$
\left|x_{1}(t)-x_{2}(t)\right|^{2} \leq \exp (2 \rho d \nu([0, t]))\left|x_{1}(0)-x_{2}(0)\right|^{2} .
$$

Uniqueness of solutions thus follows readily from the foregoing estimate, and moreover, one can obtain the bounds on the difference of the two solutions starting from two different initial conditions. One also observes that, if there is no perturbation, that is, $f(t, x) \equiv 0$, then the difference between the two trajectories at all times is bounded by the difference between their initial conditions. Uniqueness has been shown for the ZOSwP in [114] (prox-regular compact $\mathcal{S}(t)$ ), for the FOSwP in [172] (convex $\mathcal{S}(t)$ ), $[241,177,237,143]$ (prox-regular $\mathcal{S}(t)$ ), [446, 375] (prox-regular $\mathcal{S}(t)$ with bounded 
retraction), [487] (convex $\mathcal{S}(t)$ with bounded retraction), [25] (degenerate FOSwP with prox-regular $\mathcal{S}(t)$ ), [203] (integral perturbation and prox-regular $\mathcal{S}(t)$ ), [535] (set-valued perturbation), [348] (maximal monotone DIs), [410] (subdifferential DIs), [278] (EVIs of second kind). Continuous dependence holds for DIs as in (3.8) (b) with $K$ prox-regular and $f(\cdot)$ Lispchitz continuous [33, Theorem 4.2].

It is important to note that the aforementioned discussions relate to forward uniqueness as, in general, the uniqueness does not hold backward in time for the systems under consideration. To see this we revisit Example 4.

EXAMPLE 8. Consider the scalar FOSwP: $\dot{x} \in-\mathcal{N}_{[t, t+1]}(x), t \geq 0$, and $x(0) \in$ $[0,1]$. Consider the initial condition $x(0)=x_{0} \in[0,1]$. Then the solution of this system is given by

$$
x(t)= \begin{cases}x_{0}, & \text { if } 0 \leq t \leq x_{0} \\ t, & \text { if } t \geq x_{0}\end{cases}
$$

Clearly, for each $\bar{t} \geq 0$ and given $x(\bar{t})$, there is a unique value for $x(t)$ for each $t \geq \bar{t}$. However, there is no unique way to reconstruct the solution on the interval $[0, \bar{t})$ for a given $x(\bar{t})$.

5.2.5. LCS with relative degree one. An important application of the sweeping processes are the complementarity systems which have found applications in several applications. We will use these systems as a case study for results on existence of solutions. To see an application of FOSwP in the context of complementarity systems, consider the system (2.22) described by

$$
\begin{gathered}
\dot{x}=f(t, x)+B \lambda \\
0 \leq \lambda \perp z=C x+G u+H \geq 0 .
\end{gathered}
$$

Under the assumption that there exists a positive definite matrix $P \in \mathbb{R}^{n \times n}$ satisfying $P B=C^{\top}$, and by letting $z=R x$ where $R$ is the positive square root of $P$, it follows from basic convex analysis (see Section 3.4 or [143]) that (5.13) can be rewritten as $\dot{z}(t)-R f\left(t, R^{-1} z(t)\right)-R E u(t) \in-\mathcal{N}_{\mathcal{S}(t)}(z(t))$, where $\mathcal{S}(t) \triangleq\left\{x \in \mathbb{R}^{n} \mid C R x+G u(t)+\right.$ $H \geq 0\}$. This system is in the form of (5.7) and one can then impose assumptions on the mapping $\mathcal{S}(\cdot)$, which can indeed be written in terms of system data [143], to guarantee existence of solutions.

5.2.6. Other results on existence of solutions. We demonstrated two principal techniques to study existence of solutions for FOSwP. In this section, we give an overview of some results which have been obtained for more general class of systems using these techniques, and also some of the other results which are obtained from using other proof techniques.

- Extensions of Time-Stepping Algorithms: After being initially proposed for unperturbed FOSwP with convex-valued time-varying set-valued mappings, Moreau's catching-up algorithm has been used to prove existence results for several generalizations of FOSwP. We mentioned [439, 427] as classical references on this topic, but similar results can be found in [147, 209, 512, 531, 446, 445, 444, 559, 199, 116, 487]. Among extensions and similar studies, we also find discretization for different kinds of variational inequalities [27, 23, 43, 388, 462], set-valued perturbations [169, 237], state-dependent FOSwP [39, 46, 26, 170, 293, 296, 376], time-delay FOSwP and SOSwP $[235,236,117,450,174,177]$, time-dependent maximal monotone DIs [64, 153], proximal algorithms, i.e., implicit or backward Euler discretization of 
(2.16), for optimization problems [48, 57, 286, 412, 477] (it is known that an explicit Euler method has strong drawbacks [477, Sections 3.3, 6.3]). Systems with prox-regular set-valued mappings have also been studied using time-stepping algorithms [42, 186, 204, 238].

- Fixed-Point Methods: As it is done in the case of conventional dynamical systems described by ordinary differential equations, it is possible to describe the solutions of system (5.7) as the fixed point of an operator acting on the space of the solutions. Existence results based on fixed-point theory can be found in [24, 172, 241, 177].

- General regularization techniques: We presented one particular regularization technique based on Moreau-Yosida approximations which finds its origin in [434, 436], and has been used in $[54,367,340]$. Other instances of regularization methods that exist in the literature are in $[29,105,203,422,535,415,504]$, some of which use the reduction of FOSwP to compact DIs (see Section 3.3). Regularization is also used to derive Pontryagin optimality conditions in the optimal control of FOSwP, see e.g., [54].

- Particular methods for complementarity systems: For LCS, we find proofs of wellposedness based on similar discretization schemes in [299, 510]. Apart from transforming the systems in the sweeping process, the particular structure of LCS can be studied using other techniques (following an "event-driven" analysis strategy) [307]. One can also rewrite the system in frequency domain using Laplace transform and recast the Cauchy problem as rational complementarity problem [151]. These methods, specifically designed for LCS, can also handle distributional solutions which result from higher relative degree between the complementarity variables.

- Other methods: Some other proof techniques different from the above ones, have been investigated in $[486,485,363]$ for FOSwP with no perturbation, and BV solutions.

5.3. Maximal monotone DIs. The tools used in studying the solutions of FOSwP and its variants have also been useful in studying the solution concepts for DIs with maximal monotone mappings on the right-hand side. These are the inclusions modeled as

$$
\dot{x} \in-\mathcal{M}(t, x), \quad x(0) \in \operatorname{cl}(\operatorname{dom} \mathcal{M}(0, \cdot))
$$

where $\mathcal{M}(t, \cdot): \mathbb{R}^{n} \rightrightarrows \mathbb{R}^{n}$ is a maximal monotone operator. For autonomous maximal monotone DIs (which do not depend on time), one finds a rather comprehensive treatment in the books $[62,123]$. These approaches can be generalized to some extent when there is a time-dependent function entering in the dynamics additively, but extending these results to the general case, where the domain of the set-valued operator is in particular time-varying, has proved rather challenging. In [560], we see one of the first attempts to study systems with time-varying domains, where a very strong regularity assumption was imposed with respect to the pseudo-distance between two maximal operators $\mathcal{M}_{1}, \mathcal{M}_{2}$ defined as

$$
d_{\mathrm{svm}}\left(\mathcal{M}_{1}, \mathcal{M}_{2}\right) \triangleq \sup \left\{\frac{\left\langle\eta_{1}-\eta_{2}, z_{2}-z_{1}\right\rangle}{1+\left|\eta_{1}\right|+\left|\eta_{2}\right|}, \eta_{i} \in \mathcal{M}_{i}\left(z_{i}\right), z_{i} \in \operatorname{dom}\left(\mathcal{M}_{i}\right), i=1,2\right\} .
$$

It is noted that, when $\mathcal{M}(t, x)=\mathcal{N}_{\mathcal{S}(t)}(x)$ with $\mathcal{S}(t)$ closed convex, then we get $d_{\text {svm }}\left(\mathcal{M}\left(t_{1}, \cdot\right), \mathcal{M}\left(t_{2}, \cdot\right)\right)=d_{\text {Haus }}\left(\mathcal{S}\left(t_{1}\right), \mathcal{S}\left(t_{2}\right)\right)$ and hence imposing continuity on the mapping $\mathcal{M}(\cdot)$ in (5.14) was seen as a natural extension of the continuity assumption imposed on the domains in FOSwP. In particular, [560] requires the mapping 
$\mathcal{M}(t, \cdot)$ to be uniformly continuous, that is, there exists a sequence of piecewise constant operators $\mathcal{M}_{i}:[0, T] \times \mathbb{R}^{n} \rightrightarrows \mathbb{R}^{n}$ such that for each $t \in[0, T]$ one has: $\lim _{i \rightarrow \infty} d_{\mathrm{svm}}\left(\mathcal{M}\left(t_{i+1}, \cdot\right), \mathcal{M}\left(t_{i}, \cdot\right)\right)=0$. In [368] this line of work is generalized to consider systems where the regularity with respect to time can be relaxed to a BV function, in order to cover some results developed in the context of FOSwP.

However, in a recent work [153], it has been shown that working with the notion of $d_{\text {svm }}$ is restrictive for the systems of form (5.14). In particular, it is seen that continuity with respect to this particular notion of distance may not hold for LCS with time-varying terms in the complementarity relations as seen in the following example.

ExAMPle 9. Consider an LCS given by

$$
\begin{gathered}
\dot{x}(t)=B \lambda(t) \\
0 \leq \lambda(t) \perp z(t)=B^{\top} x(t)+D \lambda(t)+v(t) \geq 0,
\end{gathered}
$$

where $B=\left[\begin{array}{l}0 \\ 1\end{array}\right]$, and $D=\left[\begin{array}{cc}0 & 1 \\ -1 & 0\end{array}\right]$. Consider the set-valued mapping $\mathcal{F}: \mathbb{R}^{2} \rightrightarrows \mathbb{R}^{2}$ given by $\mathcal{F}(\zeta)=\{\eta \mid \eta \geq 0, \zeta \leq 0$, and $\langle\eta, \zeta\rangle=0\}$. It can be verified that (5.16) can be written in the form (5.14), with $\mathcal{M}(t, x) \triangleq B(\mathcal{F}+D)^{-1}\left(B^{\top} x+v(t)\right)$, where $\mathcal{M}(t, \cdot)$ is maximal monotone for each $t$. Let $v:[0, T] \rightarrow \mathbb{R}^{2}$ be an $A C$ function such that for some $t_{1}, t_{2} \in[0, T]$, we have

$$
v\left(t_{1}\right)=\left[\begin{array}{l}
0 \\
0
\end{array}\right] \quad \text { and } \quad v\left(t_{2}\right)=\left[\begin{array}{c}
-1 \\
0
\end{array}\right] .
$$

Let $\mathcal{M}_{i} \triangleq \mathcal{M}\left(t_{i}, \cdot\right)$ with $i=1,2$. It can be verified that $0 \in \mathcal{M}_{1}(\rho+1)$ and $1 \in \mathcal{M}_{2}(0)$ for any $\rho \geq 0$. From Definition 5.15, we get

$$
d_{\mathrm{svm}}\left(\mathcal{M}_{1}, \mathcal{M}_{2}\right)=\sup _{\substack{x_{1} \in \operatorname{dom}\left(\mathcal{M}_{1}\right), y_{1} \in \mathcal{M}_{1}\left(x_{1}\right), x_{2} \in \operatorname{dom}\left(\mathcal{M}_{2}\right), y_{2} \in \mathcal{M}_{2}\left(x_{2}\right)}} \frac{\left\langle y_{2}-y_{1}, x_{1}-x_{2}\right\rangle}{1+\left|y_{1}\right|+\left|y_{2}\right|} \geq \frac{\rho+1}{2} .
$$

Since the righthand side is not bounded, we can conclude that the set-valued mapping $\mathcal{M}(t, \cdot)$ is not $A C$ with respect to $d_{\mathrm{svm}}$.

This example shows that the direct extensions of sweeping-process-based results to maximal monotone operators, such as the ones reported in [368], have their limitations. However, a result on existence and uniqueness of solutions for system (5.14), which overcomes these limitations, has appeared in [153]:

THEOREM 5.8. Consider the system (5.14) and assume that the following assumptions hold:

1. For each $t \in[0, T]$, the operator $\mathcal{M}(t, \cdot)$ is maximal monotone.

2. There exists a nondecreasing function $\varphi \in A C([0, T], \mathbb{R})$ such that

$$
\sup _{z \in \operatorname{dom} \mathcal{M}(s, \cdot)} d(z, \operatorname{dom} \mathcal{M}(t, \cdot)) \leq \varphi(t)-\varphi(s), \quad \forall s, t \text { with } 0 \leq s \leq t \leq T .
$$

3. For every positive number $r$, there exists $\sigma_{r} \in \mathcal{L}^{1}\left([0, T], \mathbb{R}_{+}\right)$such that

$$
\left|\mathcal{M}^{0}(t, x)\right| \leq \sigma_{r}(t)(1+|x|)
$$

for all $x \in \mathbb{B}^{n}(r) \cap \operatorname{dom} \mathcal{M}(t, \cdot)$ with $t \in[0, T]$.

4. The set-valued mapping $t \mapsto \operatorname{graph}(\mathcal{M}(t, \cdot))$ is outer semicontinuous on $[0, T]$. 
Then, there exists a unique solution $x \in A C\left([0, T], \mathbb{R}^{n}\right)$ of (5.14).

Proof. (sketch of) The proof of this result uses similar tools as we used in proving existence of solutions to FOSwP. Let us start by partitioning the interval $[0, T]$ by choosing the time instants $t_{0}^{k}, t_{1}^{k}, t_{2}^{k}, \cdots t_{N^{k}}^{k}$, and let $h_{i+1}^{k} \triangleq t_{i+1}^{k}-t_{i}^{k}$, and $h^{k} \triangleq \max _{i} h_{i}^{k}$. In what follows, we provide the construction of an approximate solution $x_{k}:[0, T] \rightarrow$ $\mathbb{R}^{n}$ for a fixed $k$. As we increase $k$, so that $h^{k} \rightarrow 0$, the sequence $\left\{x_{k}\right\}_{k \in \mathbb{N}}$ is seen to converge and the limit is a solution. More specifically, for the chosen discrete time instants, a sequence $x_{i+1}^{k}=\left(I_{n}+h_{i+1}^{k} \mathcal{M}\left(t_{i+1}^{k}, \cdot\right)\right)^{-1}\left(x_{i}^{k}\right)$ is introduced. Recall that for each real $h>0$, the operator $\left(I_{n}+h \mathcal{M}\left(t_{i+1}^{k}, \cdot\right)\right)^{-1}$ is the so-called Yosidaapproximation of $\mathcal{M}\left(t_{i+1}^{k}, \cdot\right)$. Next, we introduce the function $\psi:[0, T] \rightarrow \mathbb{R}_{+}$as

$$
\psi(t) \triangleq t+2 \varphi(t)+(1+\gamma) \int_{0}^{t} \sigma_{r_{\gamma}}(s) d s \quad \forall t \in[0, T],
$$

which is used in constructing the sequence of approximate solutions as follows:

$$
x_{k}(t) \triangleq \frac{\psi\left(t_{i+1}^{k}\right)-\psi(t)}{\psi\left(t_{i+1}^{k}\right)-\psi\left(t_{i}^{k}\right)} x_{i}^{k}+\frac{\psi(t)-\psi\left(t_{i}^{k}\right)}{\psi\left(t_{i+1}^{k}\right)-\psi\left(t_{i}^{k}\right)} x_{i+1}^{k}
$$

where $t \in\left[t_{i}^{k}, t_{i+1}^{k}\right]$ and $i \in\left\{0,1, \ldots, N^{k}-1\right\}$. By definition, $x_{k}(\cdot)$ is a continuous function and $x_{k}\left(t_{i}^{k}\right)=x_{i}^{k}$ for all $i \in\left\{0,1, \ldots, N^{k}\right\}$. It can be then shown, under the hypothesis of the theorem, that

$$
x(t) \triangleq \lim _{\left|h^{k}\right| \rightarrow 0} x_{k}(t)
$$

is the desired solution to the inclusion (5.14). The details appear in [153].

As alluded to in Section 5.2.6, the time-discretization of maximal monotone DIs is closely linked with proximal algorithms and convex optimization [48, 57, 286, 477], the first result on this topic can be found in [412] when $\mathcal{M}(t, x)=\mathcal{N}_{K}(x), K \subset \mathbb{R}^{n}$ closed convex compact.

5.3.1. Time-varying complementarity relations. As a consequence of these general results on time-varying maximal monotone operators, we can treat the cone complementarity systems described by

$$
\begin{gathered}
\dot{x}(t)=f(t, x)+B \eta(t) \\
v(t)=C x(t)+D \eta(t)+h(t), \\
K \ni v(t) \perp \eta(t) \in K^{\star},
\end{gathered}
$$

where the primary difference compared to (5.13) is due to the presence of a matrix $D \succcurlyeq 0$, and the Lebesgue-measurable function $h:[0, \infty) \rightarrow \mathbb{R}^{m}$ in (5.19b). As a generalization, we consider $K$ to be a closed, convex polyhedral cone, while $K^{\star}$ is its dual cone (see Section A.1). We introduce the set-valued mapping

$$
\mathcal{S}(t) \triangleq\left\{z \in \mathbb{R}^{m} \mid z+h(t) \in K\right\}
$$

and reformulate the dynamics of (5.19) as

$$
\eta(t) \in-\mathcal{N}_{\mathcal{S}(t)}(C x(t)+D \eta(t)) .
$$

Because of the added structure on the set $K$, and the regularity assumption on $h(\cdot)$, it is possible to rewrite the conditions for the well-posedness of (5.19).

COROllary 5.9. [524] Consider the system (5.19) with the vector field $f(t, x)$ satisfying (A2), and suppose that 
- $D \succcurlyeq 0$ and there exists $P=P^{T} \succ 0$ such that $\operatorname{ker}\left(D+D^{T}\right) \subseteq \operatorname{ker}\left(P G-C^{T}\right)$.

- It holds that ${ }^{10} \operatorname{rint}(K-h(t)) \subseteq \operatorname{rint}\left(\operatorname{Im}\left(\partial \sigma_{K-h(t)}+D\right)\right)$ and $D K^{*} \subseteq \operatorname{Im}(C)$.

- The matrix $C$ is such that ${ }^{11} \operatorname{Im}(C)-K=\mathbb{R}^{m}$.

- For each $x \in \mathbb{R}^{n}$ and $t \geq 0$, if the set $\Lambda_{x}(t) \triangleq\left\{\eta \in K^{*} \mid v=C x+D \eta+h(t) \in\right.$ $K,\langle\eta, v\rangle=0\}$ has a nonzero element, then $\Lambda_{x}(t) \cap \operatorname{Im}\left(D+D^{T}\right) \neq \emptyset$.

If $h(\cdot)$ is locally $A C$ (respectively, $R C B V$ ), then there exists a unique weak solution to (5.19) which is continuous (resp. RCBV).

The basic idea in [524] is that these assumptions allow us to rewrite the system in the form: $\dot{z} \in f(t, z)-\mathcal{M}(t, z)$, with $\mathcal{M}(t, \cdot)$ maximal monotone for each $t$, and $f(t, \cdot)$ has some Lipschitz continuity. One can then combine the result of Theorem 5.8 and the development in Section 5.1.4 to prove this result.

5.4. Solutions of SOSwP. The particularity of FOSwP is that the regularity of solutions is determined by the regularity of the set-valued mapping $\mathcal{S}(\cdot)$, which is prespecified for the problem. However, in SOSwP and HOSwP, the jumps in the state trajectories occur when some components of the state hit the constraint surface. The discontinuity of certain state variables makes it difficult to obtain the estimates that allow us to construct converging sequences. To consider the question of existence of solutions to system subjected to the unilateral constraints described in (2.6) or (4.10), let us introduce the formal notion of solution.

Definition 5.10. A solution to the Cauchy problem (2.6) (or (4.10)) with an initial condition $\left(q_{0}, v_{0}\right) \in \Phi \times V\left(q_{0}\right)$, over an interval $I=[0, T]$, is a pair $(q, v)$ such that $v(\cdot)$ is $R C B V$ on $I ; q(t)=q_{0}+\int_{0}^{t} v(s) d s ; q(t) \in \Phi$ and $v_{e}(t) \in V(q(t))$ for all $t \geq 0$; and furthermore, there exists a positive measure $d \nu$ such that both $d t$ and $d v$ possess densities with respect to $d \nu$, denoted by $d t / d \nu$ and $d v / d \nu$ respectively, and

$$
M(q) \frac{d v}{d \nu}(t)+F(t, q, v) \frac{d t}{d \nu}(t) \in-\mathcal{N}_{V(q(t))}\left(v_{e}\right), d \nu \text {-a.e. on } I .
$$

The choice of the measure $d \nu$ is not unique since the right-hand side of $(2.6 \mathrm{c})$ is a cone. However, by Lebesgue-Radon-Nikodym Theorem, the functions $d t / d \nu(\cdot) \in$ $\mathcal{L}_{1}(I, R ; d \nu)$ and $d v / d \nu(\cdot) \in \mathcal{L}_{1}\left(I, \mathbb{R}^{n} ; d \nu\right)$ are uniquely determined for a given $d \nu$.

5.4.1. Constructing Solutions via Discretization. We here present a result which uses a time-stepping algorithm as the main tool for establishing the existence and uniqueness of the solution. For this result, the following regularity assumptions are required on the system data:

(H1) The function $F(\cdot, \cdot, \cdot)$ is continuous and is continuously differentiable $\left(\mathcal{C}^{1}\right)$ with respect to its second and third arguments.

(H2) The mapping $M(\cdot)$, from $\mathbb{R}^{n}$ to the set of symmetric positive definite matrices, belongs to class $\mathcal{C}^{1}$

(H3) For each $i \in\{1, \ldots, m\}$, the function $h_{i} \in \mathcal{C}^{1}\left(\mathbb{R}^{n}, \mathbb{R}\right)$, its gradient $\nabla h_{i}(q)$ is locally Lipschitz continuous and does not vanish in a neighborhood of $\left\{q \in \mathbb{R}^{n} \mid h_{i}(q)=0\right\}$.

(H4) The active constraints are functionally independent, i.e., $\left\{\nabla h_{i}(q)\right\}_{i \in \mathcal{I}(q)}$ is linearly independent for all $q \in \Phi$, where $\mathcal{I}(q)=\left\{i \mid h_{i}(q)=0\right\}$.

\footnotetext{
${ }^{10}$ We always have the inclusion $K-h(t)=\partial \sigma_{K-h(t)}(0) \subseteq \operatorname{Im}\left(\partial \sigma_{K-h(t)}+D\right)$. However, the set inclusions are not preserved by the rint operator in general.

${ }^{11}$ The equation $\operatorname{Im}(C)-K=\mathbb{R}^{m}$ is to be interpreted in the sense that, for each $h \in \mathbb{R}^{m}$, there exists $x \in \mathbb{R}^{n}$, and $v \in K$, such that $h=C x-v$.
} 
(H5) For each $q \in \Phi$, for all $(i, j) \in \mathcal{I}(q) \times \mathcal{I}(q), i \neq j$,

$$
\begin{cases}\left\langle\nabla h_{i}(q), M^{-1} \nabla h_{j}(q)\right\rangle \leq 0, & \text { if } e=0, \\ \left\langle\nabla h_{i}(q), M^{-1} \nabla h_{j}(q)\right\rangle=0, & \text { if } e \in] 0,1] .\end{cases}
$$

Under these conditions, we consider a sequence of solutions using the time discretization principle. Let $h>0$ be a given time-step, set $q_{i+1}^{k}=q_{i}^{k}+h^{k} v_{i}^{k}$, and the velocity $v_{k}(\cdot)$ is obtained via

$$
M\left(q_{i+1}^{k}\right)\left(\frac{v_{i+1}^{k}-v_{i}^{k}}{h^{k}}\right)+F\left(t_{i+1}^{k}, q_{i+1}^{k}, u_{i}^{k}\right) \in-\mathcal{N}_{V\left(q_{i+1}^{k}\right)}\left(\frac{v_{i+1}^{k}+e v_{i}^{k}}{1+e}\right),
$$

which is an extension of Moreau's catching-up algorithm used for the FOSwP. The elements of the set in the right-hand side of (5.23), are approximations of the contact forces impulses, see [4, Chapter 10] [130, Section 5.7.3]. Hence they are bounded variables, even at impact times. Using (A.6) we can write $v_{i+1}^{k}$ more explicitly as

$$
v_{i+1}^{k}=-e v_{i}^{k}+(1+e) \operatorname{proj}_{M\left(q_{i+1}^{k}\right)}\left(V\left(q_{i+1}^{k}\right) ; v_{i}^{k}+\frac{h^{k}}{1+e} M^{-1}\left(q_{i+1}^{k}\right) F\left(t_{i+1}^{k}, q_{i+1}^{k}, v_{i}^{k}\right)\right) .
$$

As a result, let us consider the sequence of approximate solutions $\left\{q_{k}, v_{k}\right\}_{k \in \mathbb{N}}$ defined on the interval $[0, T]$ as follows:

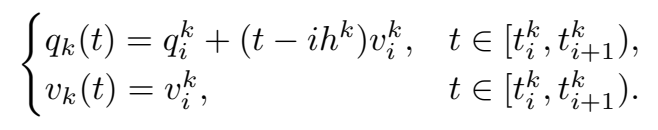

The following result shows the existence of solutions, locally in time, on an interval $[0, \tau]$ which depends only on the data and does not depend on the approximate solutions. This local behavior is due to the fact that the nonlinearities $M(\cdot)$ and $F(t, \cdot, \cdot)$ are only assumed to be locally Lipschitz continuous, and thus the finite escape times may occur even if the solution trajectories do not hit the constraint boundary.

THEOREM 5.11. Consider the initial value problem (2.6) under the hypotheses (H1)-(H5). Then there exist a maximal interval $[0, \tau], \tau \leq T$, and $(q, v) \in$ $\mathcal{C}^{0}\left([0, \tau], \mathbb{R}^{n}\right) \times R C B V\left([0, \tau], \mathbb{R}^{n}\right)$ such that a subsequence of $\left\{q_{k}, u_{k}\right\}_{k \in \mathbb{N}}$ defined in (5.24) such that $q_{k} \underset{\mathcal{C}^{0}\left([0, \tau], \mathbb{R}^{n}\right)}{\text { strongly in }} q$ and $u_{k} \rightarrow u$ except possibly on a countable set, and $(q, v)$ is the solution to $(2.6)$.

5.4.2. Continuity of solutions with respect to system data. The foregoing result shows that a sequence of approximate solutions converges to the actual solution of the system under the hypotheses (H1)-(H5), and hence continuity of solutions w.r.t. initial data also holds (in the single constraint one degree-of-freedom case, this property was shown in [499, Theorem 5.1]). However, this continuity comes at the price of restrictions imposed on the geometry of constraints in (H5), which requires the boundaries of the configuration space to intersect orthogonally, or to make an acute angle in the kinetic metric [130, Section 6.2.7]. Indeed, if such orthogonality is not imposed, we do not get continuity with respect to initial conditions. As an example, consider two types of geometrical configurations between two linear constraints in Figure 5.2, with restitution coefficient $e=1$. The constraints in Figure 5.2a do not respect (H5). For some $\varepsilon_{1}, \varepsilon_{2}>0$, consider three sets of initial condition with same initial velocity, i) on the bisector, denoted by $\left(q_{1}, q_{2}\right)$, ii) left side of the bisector 
$\left(q_{1}-\varepsilon_{1}, q_{2}+\varepsilon_{2}\right)$, and iii) right-side of the bisector $\left(q_{1}+\varepsilon_{1}, q_{2}-\varepsilon_{2}\right)$, which can be made arbitrarily close to each other by choosing $\varepsilon_{1}$ and $\varepsilon_{2}$ small enough. The resulting trajectories however diverge away from each other in different directions after the impact. The situation however does not occur when the constraints are orthogonal to each other in Figure 5.2b.

5.4.3. Other results for Lagrange systems and SOSwP. Well-posedness analysis for complementarity mechanical systems started in 1959 with Bressan's counterexample to uniqueness [120], then again in 1978 [498] (in 1985 for the SOSwP [426]), and was still the object of investigations almost fourty years later [465, 466]. The result presented in Theorem 5.11 is a strong result in the sense it shows continuity of solutions with respect to initial data. There are results on existence of solutions for SOSwP, based on other techniques, which establish existence and uniqueness of solutions without any claims about continuity with respect to initial data [73]; However, such works assume the analyticity of data to guarantee the uniqueness and avoid Bressan's pathological counter-examples [130, Section 2.4.3] (analycity is used also in [499] [473, Theorem 3.7] [474]). The earlier works on SOSwP were concentrated on single constraint (so that (H5) holds trivially). In these works, we see approaches based on Yosida-Moreau regularization [498, 467], and time-stepping schemes [426, 427, 403, 233, 234]. Extensions to systems with dry Coulomb friction have also been considered, see [519, 75] for earlier references, and more recently using time-stepping methods in $[465,466]$. Painlevé paradoxes, which are some kind of singularity $[130,265,182]$, are a source of major difficulty in the analysis of systems with set-valued friction. The results about maximal monotone DIs do not apply directly, because of the mass matrix inverse that ususally destroys the monotonicity of $M(q)^{-1} \partial \varphi(\dot{q})$.

5.5. Higher order sweeping processes. Following the template of Section 2.1, we now address the solutions of HOSwP. In moving from FOSwP to SOSwP, we saw that static constraints on the position variable translate into constraints on the velocity variable with possible discontinuities (depending on the state variable). In HOSwP, one basically describes constraints on the higher derivatives of a component of the state variable (which has static constraints). For such systems, we restrict our attention to the linear dynamics described in (2.9) and their solution has to be interpreted in the following sense: Find nonnegative real-valued Radon measures

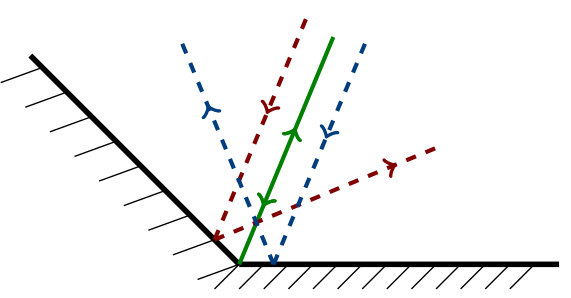

(a) Loss of continuity in non-orthogonal constraints.

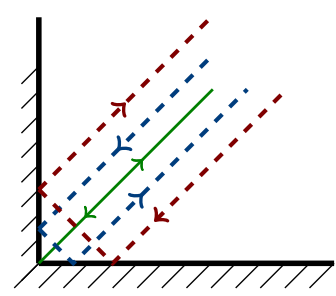

(b) Continuity holds when the constraints are orthogonal.

Fig. 5.2: Graphical illustration of continuity with respect to initial condition in systems with multiple constraints. 
$d \nu_{i}$ relative to which the Lebesgue measure $d t$ and the Stieltjes measure $d z_{i}$ possess densities $\frac{d t}{d \nu_{i}}$ and $\frac{d z_{i}}{d \nu_{i}}$ respectively such that, for $1 \leq i \leq r-1$

$$
\frac{d z_{i}}{d \nu_{i}}-z_{i+1}(t) \frac{d t}{d \nu_{i}}(t) \in-\mathcal{N}_{\mathcal{T}_{\Phi_{m}}^{i-1}\left(Z_{i-1}\left(t^{-}\right)\right)}\left(z_{e, i}(t)\right), d \nu_{i} \text { a.e. } t \in[0, T]
$$

and

$$
\left(C A^{r-1} B\right)^{-1}\left[\frac{d z_{r}}{d \nu_{r}}-C A^{r} W^{-1} z(t) \frac{d t}{d \nu_{r}}(t)\right] \in-\mathcal{N}_{\mathcal{T}_{\Phi_{m}}^{r-1}\left(Z_{r-1}\left(t^{-}\right)\right)}\left(z_{e, r}\left(t^{+}\right)\right)
$$

The following result is borrowed from [6] and uses the structure of the system combined with analysis over an appropriately chosen distributional solution space.

TheOREM 5.12. Consider system (2.9) with $m=1$. If $C A^{r-1} B>0$, then there exist locally $R C B V$ functions $z_{i}$ and Radon measure $d \nu_{i}, 1 \leq i \leq r$ that satisfy (5.25). The function $\zeta(\cdot)$ satisfying $(2.8 \mathrm{c})$ is locally AC. These results extend to $m \geq 2$ with the sufficient condition that $C A^{r-1} B$ be a Stieltjes matrix.

5.5.1. Constructing Discretized Solutions. As done in the previous section, the discrete approximations of the solutions are constructed by a sequence of piecewise continuous functions. To define the $N$-th element of this sequence, we consider the points $t_{0}=0, t_{j} \in[0, T], 1 \leq j \leq N-1, t_{N}=T$, so that the interval $[0, T]$ is split in $N$ intervals of the form $\left[t_{j}, t_{j+1}[, 0 \leq j \leq N-1\right.$. For this fixed partition, the corresponding discrete solution is given by $x^{N}:\left[0, T\left[\rightarrow \mathbb{R}^{n}\right.\right.$, where $x^{N}(t)=$ $\left[z^{N^{\top}}(t), \zeta^{N^{\top}}(t)\right]^{\top}$. The functions $z^{N}(\cdot)$ and $\zeta^{N}(\cdot)$ are obtained by setting $z^{N}(t)=z_{j}^{N}$, and $\zeta^{N}(t)=\zeta_{j}^{N}$, for $t \in\left[t_{j}, t_{j+1}\left[\right.\right.$; the vectors $z_{j}^{N}$ and $\zeta_{j}^{N}$ are obtained by the following algorithm: $z_{j}^{N}=\left[z_{1, j}^{N}, \ldots, z_{r, j}^{N}\right]^{\top}$, with the points $z_{i, j}^{N} \in \mathbb{R}, 1 \leq i \leq r, 0 \leq j \leq N-1$ obtained by

$$
z_{i, j+1}^{N}-z_{i, j}^{N}-h z_{i+1, j+1}^{N}=\nu_{i, j+1}^{N} \in-\mathcal{N}_{\mathcal{T}_{\Phi}\left(Z_{i-1, j}^{N}\right)}\left(z_{i, j+1}^{N}\right), \quad 1 \leq i \leq r-1
$$

while using the notation $Z_{i, j}^{N}=\left(z_{1, j}^{N}, z_{2, j}^{N}, \ldots, z_{i, j}^{N}\right)$, and

$$
\begin{gathered}
z_{r, j+1}^{N}-z_{r, j}^{N}-h C A^{r} W^{-1} z_{j+1}^{N}=C A^{r-1} B \nu_{r, j+1}^{N}, \\
\nu_{r, j+1}^{N} \in-\mathcal{N}_{\mathcal{T}_{\Phi}^{r-1}\left(Z_{r-1, j}^{N}\right)}\left(z_{r, j+1}^{N}\right) .
\end{gathered}
$$

For the zero dynamics, we use for the sake of simplicity a backward Euler scheme: $\zeta_{j+1}^{N}-\zeta_{j}^{N}=h\left(A_{0} \zeta_{j+1}^{N}+B_{0} z_{1, j+1}^{N}\right)$, and the vector of discretized multipliers by $\nu_{j+1}^{N}$, that is, $\nu_{j+1}^{N}=\left[\nu_{1, j+1}^{N}, \ldots, \nu_{r, j+1}^{N}\right]^{\top}$. Then the discrete-time system can be rewritten compactly as

$$
x_{j+1}^{N}-x_{j}^{N}=h W A W^{-1} x_{j+1}^{N}+G \nu_{j+1}^{N}
$$

where we recall that $G=\left[\begin{array}{cc}I_{(r-1) \times(r-1)} & 0 \\ 0 & C A^{r-1} B \\ 0 & 0\end{array}\right]$. Consider the step function $\nu_{N}$ : $[0, T] \rightarrow \mathbb{R}^{r}$ such that $\nu^{N}(t)=\nu_{j}^{N}$, for $t \in\left[t_{j}, t_{j+1}\left[\right.\right.$ and $\nu^{N}(T)=\nu_{N}^{N}$.

Proposition 5.13. Assume that the triplet $\left(W A W^{-1}, G, H\right)$ is observable, controllable and positive real. Then the following statements hold:

- $C A^{r-1} B>0$ and

- There exists a subsequence $\left\{x^{N_{k}}\right\}$ of $\left\{x^{N}\right\}$ which converges pointwise to an $R C B V$ function $x:[0, T] \rightarrow \mathbb{R}^{n}$, and a subsequence $\left\{\nu^{N_{k}}\right\}$ of $\left\{\nu^{N}\right\}$ which converges pointwise to an $R C B V$ function $\nu:[0, T] \rightarrow \mathbb{R}^{r}$. 
5.5.2. LCS with higher relative degree. So far, we have considered LCS under the passivity assumption, which have an underlying transfer matrix of index 1 (see Definition C.1 and Theorem D.2). Some results on solutions of complementarity systems with relative degree greater than 1 can be obtained by using the framework of HOSwP. To see this, consider the system (2.22). If this system has a relative degree $r$, and $C A^{r-1} B>0$, then by letting $z_{1}=C x$, it is possible to rewrite the system in the form (2.8).

Corollary 5.14. Consider system (2.22) with the matrices $D, E, F, G, H$ to be identically zero, and assume that the system has a relative degree $r$, and $C A^{r-1} B>0$. Then, the system has a unique solution in the class of piecewise smooth distributions. Results in the multivariable case $(m>1)$ can be found in [6, 132] and in [307] with a narrower class of distribution solutions. They all rely on some kind of positivity of the leading Markov parameter (Stieltjes or P-matrix).

5.6. Extensions and Perspectives. To conclude this section, we highlight some elements of the ongoing research in the area of existence of solutions for the nonsmooth systems being studied in this article.

- Extensions involving Complementarity Systems: For LCS, the choice of functional space of solutions depends on the relative degree of the complementarity variables [6, Proposition 3, Corollary 2] [307, 151, 155]. Roughly speaking, solutions are Schwartz' distributions with degree $\leq r$. See $[159,136,143]$ for LCS via FOSwP or unilateral DIs with maximal monotone operators (Section 2.2). Existence or non-existence of accumulation of events (Zeno states) is analysed in [298]. Extensions of LCS may be found in $[135,136,154]$. For NLCS $(2.24)$, local analytic solutions are used in [549], global AC or right-continuous LBV (hence admitting state jumps) solutions in [143], see also [550] for a class of cone NLCS with solutions in $\left.W^{1,2}([a, b]) ; \mathbb{R}^{n}\right)$ and integrable $\lambda$. Relaxing the assumptions of Corollary 5.9 should be tackled. MLCS and MLNCS as in (2.28) (2.25) and (2.27) deserve attention, both for the well-posedness and the numerical analyses.

- Well-posedness results for control problems: The recent trend in the research on the class of nonsmooth systems relates to addressing control problems, for example, feedback stabilizing control [429, 524], state-estimators [522, 523], or optimal control related problems $[54,144,200,201,202,207,316]$. In all these works, one sees novel type of interconnections of nonsmooth systems arise and generalizing the scope of current results to such systems is one possible direction for research. Also, for optimal control related problems, since we are essentially dealing with stateconstrained systems, the application of Pontryagin's maximum principle to get (necessary conditions) adjoint equations for optimal trajectories and control, seems to be in the form of HOSwP with two-point boundary values [128], instead of the initial value problems discussed in this article. Addressing solutions to such systems definitely requires some novel tools and methodology.

- Despite of the fact that the systems we deal with are basically nonsmooth, their solutions can be arbitrarily smooth in some cases, as shown in [497] for the PDS in (2.31), depending on the smoothness of $\mathrm{bd}(K)$ and of $f(x)+g(t)$. Thus, it is of interest analyze the structures which contribute to regularity of the solution.

REMARK 23 (Infinite-dimensional case). Apart from time-independent maximal monotone DIs that were largely aimed at treating infinite dimensional problems [123], several extensions of the foregoing finite-dimensional formalisms towards infinite dimensions have been published, mainly for the FOSwP and EVI in Hilbert spaces, see e.g., [289, 290, 28, 395, 394, 37, 196, 235], where compactness assumption on $\mathcal{S}(t)$ 
becomes crucial to guarantee the convergence of subsequences of bounded sequences of continuous maps. See also [195, 327, 197] for PDS.

6. Stability of equilibria. This section mainly deals with the extension of the absolute stability problem [139, Section 3.13] to the above set-valued systems. Here we do not deal with bifurcations and chaos analysis, see [384] and [224] for complete analyses of this topic. Before presenting some results, we highlight some peculiar features for the systems modeled by (1.1), which are not shared by smooth systems.

1. Nonsmooth systems as the above ones, may be unstable while the corresponding unconstrained system (obtained setting $w=0$ in (1.1)) is asymptotically stable $[584,280]$. In particular this occurs when the equilibria are on the admissible domain boundary. Therefore, stability analysis needs to be adapted accordingly.

2. Jump-times mismatch in systems with state jumps: two arbitrarily close trajectories outside jumps, become distant (in the usual metric) in the jump instant neighborhood [130, Section 1.3.2.3].

3. Accumulations of events, also known as Zeno behaviour (impacts, frictional stick/slip transitions, switches in some sliding-mode control systems [4, section 6.3.2], switches between complementarity modes in LCS) often occur. In some particular cases (LCS and DVIs with $D$ a P-matrix, or NLCP with strongly regular solutions $[156,509,298,461]$, mechanical systems with conservative impacts [74], some PWL systems [534, 532]), solutions can be shown to be Zeno-free.

4. Constrained state in closed, moving or fixed set, with changing vector field on the set boundary (due to the existence of the multiplier $w$ in (1.1)).

5. Changing dimension (with added or deactivated constraints) after possible accumulation of events (impacts, stick/slip), or creation of a sliding motion, occurring in finite-time: the underlying mechanism is the existence of a multiplier which takes care of the switch between the two dynamics (in Mechanics with frictionless contact this is a normal contact force, in case of friction this is a tangential contact force inside the Coulomb's cone, in circuits this is a combination of voltages and currents, in sliding-mode with attractive surface, this is a control action that is a selection of the set-valued controller).

6. Continuity of solutions w.r.t. initial data, does not always hold (like in mechanical systems with multiple constraints [130, 464], see Figure 5.2). This raises a difficulty in applying invariance results which often use such continuity as a key assumption to guarantee positive invariance of limit sets.

7. Reversibility, or uniqueness in backward time, may be lost (think of a ball bouncing on a ground, with zero restitution: an infinite number of trajectories leads in finite time to the static equilibrium). See also Example 8.

8. Forward uniqueness of solutions often holds (see Sections 5.2.4 and 5.9, Theorem 5.8, Corollary 5.9), in which case the notions of weak and strong stability are no longer relevant.

It is noteworthy also that several of these features as well as the basic characteristics of our systems, often preclude the use of stability criteria developed for other types of DIs, see e.g., [62, 70, 513] (compact, locally bounded right-hand sides), [274, 513] (closed strict convex processes, see Section 3.13).

6.1. Characterization of equilibria. General criteria exist for the existence of equilibria in DIs [62, Chapter 6, Section 2]. Concerning our systems, fixed points of (1.1) are solutions of the generalized equation (GE) (see Section A.2.2 for definition). 
More precisely, we say that $\left(x^{\star}, w^{\star}\right)$ is an equilibrium if

$$
\left\{\begin{array}{l}
0=f\left(t, x^{\star}\right)+G\left(t, x^{\star}\right) w^{\star}(t) \\
w^{\star}(t) \in-\mathcal{F}\left(t, H x^{\star}, D w^{\star}\right) .
\end{array}\right.
$$

Such a GE may take various forms, depending on the considered subclass of systems. The analysis of (6.1) and the computation of its solutions, require more specific information about $f(\cdot), G(\cdot)$ and $\mathcal{F}(\cdot)$ so that suitable equivalent formulations may be found. The relationships between the formalisms studied in Section 3, can be used to obtain different equivalent GEs. Thus the GE (6.1) may be a complementarity problem (LCP, LCCP, NLCP, MLCP, see Section B), a VI (of first kind, second kind, a QVI, see Section A.2.2), an inclusion into a normal cone or a projection (consider, e.g., PDS in (3.8) and (2.31)), and so on. In order to clarify the existence and uniqueness of solutions for such GEs, we consider few examples.

6.1.1. Projected dynamical systems. Let us start with a characterization of equilibrium points for PDS formalism given in (2.31) and (3.8).

Corollary 6.1. Let the hypotheses of Theorem 3.3 hold. The PDS formalisms in (2.31) and (3.8) give rise to equivalent GEs that characterize their fixed points $\left(x^{\star}, \lambda^{\star}(t)\right)$ :

$$
\begin{aligned}
& (a) 0 \in f\left(x^{\star}\right)+g(t)+\mathcal{N}_{\mathcal{T}_{K}\left(x^{\star}\right)}(0) \Longleftrightarrow(b) 0 \in f\left(x^{\star}\right)+g(t)+\mathcal{N}_{K}\left(x^{\star}\right) \\
& \Longleftrightarrow(c) x^{\star} \in K \text { and }\left\langle f\left(x^{\star}\right)+g(t), y-x^{\star}\right\rangle \geq 0, \forall y \in K \\
& \Longleftrightarrow(d)\left\{\begin{array}{l}
0=f\left(x^{\star}\right)+g(t)+\nabla h\left(x^{\star}\right) \lambda^{\star}(t) \\
0 \leq-h\left(x^{\star}\right) \perp \lambda^{\star}(t) \geq 0
\end{array}\right. \\
& \Longleftrightarrow(e) 0=\operatorname{proj}\left[\mathcal{T}_{K}\left(x^{\star}\right) ;-f\left(x^{\star}\right)-g(t)\right] \\
& \Longleftrightarrow(f) 0=-f\left(x^{\star}\right)-g(t)-\operatorname{proj}\left[\mathcal{N}_{K}\left(x^{\star}\right) ;-f\left(x^{\star}\right)-g(t)\right] \\
& \Longleftrightarrow x^{\star} \in K \text { and }\left\langle f\left(x^{\star}\right)+g(t), y\right\rangle \geq 0, \text { for all } y \in \mathcal{T}_{K}\left(x^{\star}\right) .
\end{aligned}
$$

The proof follows from Proposition 3.2 and Theorem 3.3. Which one of these GEs is the most convenient for analysis ? Existence of solutions can be stated directly using [240, Corollary 2.2.5]: if $F_{t}: x \mapsto f(x)+g(t)$ is continuous for each $t$ and $K$ is compact convex, then (6.2) (b) has a non empty compact set of solutions for each $t$. Uniqueness for each $t$ is guaranteed by monotonicity conditions on $F_{t}(\cdot)$ [240, Theorem 2.3.3], which is coherent with Theorem 3.3's hypothesis. More generally maximal monotonicity is a quite convenient property to characterize zeroes of GEs, and proximal-point algorithms can compute them [97, Section 23.4]. In general, a stationary equilibrium will exist only if $g(t)=0$ for all $t$, implying $\lambda^{\star}$ constant. However let us consider (6.2) (d), with $h\left(x^{\star}\right)=0$. Then $\mathcal{N}_{K}\left(x^{\star}\right)=\nabla h\left(x^{\star}\right) \lambda^{\star}$, hence $f\left(x^{\star}\right)+g(t)=-\nabla h\left(x^{\star}\right) \lambda^{\star}$. Let $g(t) \in \operatorname{Im}\left(\nabla h\left(x^{\star}\right)\right)$ for some $x^{\star}$, then there exists $\lambda^{\star}(t)$ such that $\nabla h\left(x^{\star}\right) \lambda^{\star}(t)=-g(t)$. If $f\left(x^{\star}\right)=0, x^{\star}$ is an equilibrium point which satisfies (6.2) (d) for all $t$.

6.1.2. Linear Complementarity Systems. Next, let us consider an MLCS as in (2.25). Equilibria have to satisfy the MLCP:

$$
\left\{\begin{array}{l}
A x^{\star}+B_{1} \lambda_{1}^{\star}+B_{2} \lambda_{2}^{\star}+E_{1} u(t)+E_{2}=0 \\
A_{1} \lambda_{1}^{\star}+A_{2} \lambda_{2}^{\star}+E_{5} x^{\star}+E_{6}=0 \\
0 \leq \lambda^{\star} \perp z^{\star}=C x^{\star}+D_{1} \lambda_{1}^{\star}+D_{2} \lambda_{2}^{\star}+E_{3} u(t)+E_{4} \geq 0 .
\end{array}\right.
$$


which is equivalently rewritten with obvious notation as:

$$
\left\{\begin{array}{l}
\tilde{A} x^{\star}+\tilde{B} \lambda^{\star}+\tilde{u}(t)+\tilde{E}_{2}=0 \\
0 \leq \lambda^{\star} \perp C x^{\star}+\tilde{D} \lambda^{\star}+E_{3} u(t)+E_{4} \geq 0
\end{array}\right.
$$

The tools in [240, Exercise 1.8.10] (if $C=0$ and $\tilde{D}=-\tilde{B}^{T}$ ), or in [11] (if $\tilde{D}=0$ and $P \tilde{B}=C^{T}$ for some $P=P^{T} \succ 0$, which allows one to transform the MLCP into an inclusion as in (A.6)) can be used to analyse the existence and uniqueness of solutions to (6.4). The equilibria of the relay system in (2.35) are solutions of a similar, simpler inclusion (using inversion tools as in Section A.2.4: $\operatorname{sgn}^{-1}(\cdot)=$ $\left.\left(\partial \sigma_{[-1,1]}\right)^{-1}(\cdot)=\mathcal{N}_{[-1,1]}(\cdot)\right)$.

6.1.3. Systems with Relative degree 2. As a third example, let us consider the $\operatorname{SOSwP}(4.10)$. Equilibria $(q, v)=\left(q^{\star}, 0\right)$ satisfy the GE: $g\left(q^{\star}\right)-E u(t) \in$ $-\mathcal{N}_{V\left(q^{\star}\right)}(0)$, which is equivalent (using (B.1) and the fact that $0 \in V(q)$ for all $q \in \Phi$ since $V(q)$ is a cone) to the GE: $g\left(q^{\star}\right)-E u(t) \in-\mathcal{N}_{\Phi}\left(q^{\star}\right)$. The well-posedness of this GE can be analysed via [240, Corollary 2.2.5, Theorem 2.3.3]. The set $-\mathcal{N}_{\Phi}\left(q^{\star}\right)$ contains generalized contact forces $\nabla h\left(q^{\star}\right) \lambda^{\star}(t)$. Similarly to the PDS in (6.2) (d), the multipliers $\lambda^{\star}(t)$ may be time-varying while $q^{\star}$ is constant. Assume that $\operatorname{Im}(E) \subseteq \operatorname{Im}\left(\nabla h\left(q^{\star}\right)\right)$, and $g\left(q^{\star}\right) \in \operatorname{Im}\left(\nabla h\left(q^{\star}\right)\right)$ for some $q^{\star}$. The GE can be rewritten as: find $q^{\star}$ and $\lambda^{\star}(t)$ such that $g\left(q^{\star}\right)=\nabla h\left(q^{\star}\right)\left(\eta(t)+\lambda^{\star}(t)\right), E u(t)=\nabla h\left(q^{\star}\right)(\eta(t))$, which has a solution. This is a static equilibrium, which boils down in the simplest one degree of freedom case with $\Phi=\{q \in \mathbb{R} \mid q \geq 0\}$, to $\lambda^{\star}(t)=-u(t)-m g, q^{\star}=0$, where $m>0$ is the mass and $g>0$ is the gravity acceleration. Equilibria of unilaterally constrained Lagrangian systems are studied in [501], splitting strongly active constraints $\left(\lambda_{i}^{\star}>0\right)$ and weakly active ones $\left(\lambda_{i}^{\star}=0\right)$. In the latter case, examination of the derivatives of the null multipliers and of the gap functions $h_{i}(q)$ (in a way similar to what is exposed in Section 2.4.1, see Remarks 3 and 4), allows one to construct an MLCP, whose analysis allows to determine some kind of stability of the equilibrium (i.e., what happens in a right neighborhood of $t$ if $t$ is the time at which the equilibrium is analysed). The fixed points of the HOSwP are analysed in [132], under a passivity condition.

6.1.4. Further remarks. The settings of VIs $[12,277,11,278,279]$ and GEs $[21$, $19,18,15]$, are used to characterize fixed points in circuits with set-valued components. The formalisms of PDS [178, 195, 196, 251, 291, 570, 468, 584, 327, 285, 327] and DVIs [188] are used for games, Nash equilibria, and traffic models. Moreover, GEs are used in [218] for cellular neural networks equilibria, a mixed LCP is used in [579] for equilibria of instant model predictive control. The Aubin/Lipschitz property of GEs with parameters (which characterizes how solution sets of GEs vary with parameters) is studied in $[18,15]$ when $\mathcal{F}(\cdot)$ in $(6.1)$ is a subdifferential. Contact mechanics equilibria without Coulomb friction are studied in [131] [130, Section 7.5.2], and with Coulomb friction in $[76,83,82,330,482]$, where the GEs for equilibria are analysed in great detail. In the latter setting, the equilibria usually are not unique (sets of equilibria exist) due to the set-valuedness of friction. Starting from (4.9a) and adding to the right-hand side the contribution of two-dimensional friction written as $H_{\mathrm{t}}(q) \lambda_{\mathrm{t}}$, with $\lambda_{\mathrm{t}, i} \in-\mu_{i} \lambda_{\mathrm{n}, i} \operatorname{sgn}\left(v_{\mathrm{t}, i}\right), v_{\mathrm{t}, i}=H_{\mathrm{t}, i}(q)^{T} \dot{q}[130$, Section 5.5], one ends up with the 
GE: Find $q^{\star}, \lambda_{\mathrm{n}}^{\star}(t)$ and $\lambda_{\mathrm{t}}^{\star}(t)$ such that:

$$
\left\{\begin{array}{l}
\overbrace{0 \in g\left(q^{\star}\right)-E u(t)+\nabla h\left(q^{\star}\right) \mathcal{N}_{\mathbb{R}_{m_{u}}^{+}}\left(h\left(q^{\star}\right)\right)}^{\text {frictionless GE }}+H_{\mathrm{t}}\left(q^{\star}\right) \lambda_{\mathrm{t}}^{\star}(t) \\
\lambda_{\mathrm{n}}^{\star}(t) \in-\mathcal{N}_{\mathbb{R}_{+}^{m_{u}}}\left(h\left(q^{\star}\right)\right) \\
\frac{\lambda_{\mathrm{t}, i}^{\star}(t)}{\lambda_{\mathrm{n}, i}^{\star}(t)} \in\left[-\mu_{i}, \mu_{i}\right], 1 \leq i \leq m_{u} .
\end{array}\right.
$$

One can use Proposition A.4 to rewrite $\nabla h\left(q^{\star}\right) \mathcal{N}_{\mathbb{R}_{+}^{m_{u}}}\left(h\left(q^{\star}\right)\right)=\mathcal{N}_{\Phi}\left(q^{\star}\right)$ with $\Phi=\{z \in$ $\left.\mathbb{R}^{\frac{n}{2}} \mid h(z) \geq 0\right\}$ (see Remark 19). It is possible to extend (6.5) to more general setvalued, three dimensional friction models. See [240, Section 1.4.6] for a formulation as an optimization problem and the "maximum dissipation principle". Even when $\mu_{i}=0$ for all $i$, the existence and uniqueness of solutions to (6.5) may not be trivial when $\Phi$ is non convex (see [240, Corollary 2.2.5, Theorem 2.3.3] in the compact convex case, [345] in the non convex, finitely represented case).

REMARK 24 (Periodic solutions). Existence of periodic trajectories has been studied for locally PDS (2.31) [195, 192] (for instance, the fixed point theorem [192, Theorem 3.4] relies on the monotonicity of $f(\cdot)$ in (2.31)), which by (3.8) (a) may be seen as an extension of [240, Corollary 2.2.5]), PWL systems with saturation functions (giving rise to MLCS similar to (2.25)) [508], LCS as in (2.22) [322], perturbed $F O S w P$ with fixed $\mathcal{S}$ [343] and time-varying convex or prox-regular $\mathcal{S}(t)$ [172, 241, 344] (where the strong monotonicity of $f(t, \cdot)$ in (2.2) is the central assumption), maximal monotone DIs [22], upper semicontinuous DIs [62, p. 237]. Many studies have been dedicated to periodic trajectories in mechanical systems with few degrees of freedom, mainly focussing on their stability [380, 384, 224], see [68] for existence.

6.2. Sufficient Lyapunov Conditions. Let us now provide brief insights on Lyapunov stability for evolution VIs of first and second kind (with continuous solutions), and nonsmooth mechanical systems.

6.2.1. First order case. For time-invariant first order systems, for which the solutions are AC, it is useful to write the system class abstractly as

$$
\dot{x} \in \mathcal{A}(x), \quad x(0) \in \operatorname{dom}(\mathcal{A}),
$$

so that $\mathcal{A}(\cdot)$ in (6.6) denotes the right-hand side of the DI in (1.1). The following assumptions are assumed whenever (6.6) is referred in the remainder of the text:

1. The domain of $\mathcal{A}(\cdot)$ is closed.

2. For each $x_{0} \in \operatorname{dom}(\mathcal{A})$, there exists an AC solution $x:[0, T] \rightarrow \operatorname{dom}(\mathcal{A})$ for each $T>0$, and its value at time $t \in[0, T]$ is denoted as $x\left(t ; x_{0}\right)$.

3. The origin, $\{0\}$ is in $\operatorname{dom}(\mathcal{A})$ and is an equilibrium, i.e., $x(t ; 0)=0$, for every $t \geq 0$.

In (6.1), we mentioned that the equilibria of (1.1) are of the form $\left(x^{\star}, w^{\star}\right)$. In what follows, we only consider time-invariant systems, and consider stability of the equilibrium point with $x^{\star}=0$, so that (6.6) represents the system obtained by substituting $w^{\star}$ as a function of $x^{\star}=0$.

Definition 6.2. Consider that the equilibrium point $\{0\} \in \operatorname{dom}(\mathcal{A})$ for $(6.6)$. The origin is Lyapunov stable if for every $\varepsilon>0$ there exists $\delta>0$ such that

$$
x_{0} \in \operatorname{dom}(\mathcal{A}),\left|x_{0}\right| \leq \delta \Rightarrow\left|x\left(t ; x_{0}\right)\right| \leq \varepsilon, \forall t \geq 0 .
$$

The origin is attractive if there exists $\delta>0$ such that

$$
x_{0} \in \operatorname{dom}(\mathcal{A}),\left|x_{0}\right| \leq \delta \Rightarrow \lim _{t \rightarrow+\infty}\left|x\left(t ; x_{0}\right)\right|=0 .
$$


The origin is globally attractive if the implication holds for arbitrary $\delta>0$. The origin is (globally) asymptotically stable if it is stable and (globally) attractive. The origin is globally exponentially stable if there exists $c_{0}>0$ and $\alpha>0$ such that $\left\|x\left(t ; x_{0}\right)\right\| \leq c_{0} e^{-\alpha t} x_{0}$, for every $x_{0} \in \operatorname{dom}(\mathcal{A})$.

We can now formulate a stability theorem in terms of a Lyapunov function.

THEOREM 6.3. Consider system (6.6). Suppose that there exists a continuously differentiable $V: \mathbb{R}^{n} \rightarrow \mathbb{R}$ such that $V(\cdot)$ is positive definite on $\operatorname{dom}(\mathcal{A})$, and

$$
\max _{\xi \in \mathcal{A}(x)}\langle\nabla V(x), \xi\rangle \leq-W(x), \quad \forall x \in \operatorname{dom}(\mathcal{A})
$$

for some continuous function $W: \mathbb{R}^{n} \rightarrow \mathbb{R}$. If $W(x) \geq 0$, for each $x \in \operatorname{dom}(\mathcal{A})$, then $\{0\}$ is Lyapunov stable. Furthermore, if $W(\cdot)$ is positive definite on $\operatorname{dom}(\mathcal{A})$, then $\{0\}$ is asymptotically stable.

Proof. First we prove Lyapunov stability while working with $W \equiv 0$. For a given $\varepsilon>0$, choose $\varepsilon^{\prime} \in(0, \varepsilon]$ such that $\mathcal{B}_{\varepsilon^{\prime}} \triangleq\left\{x \in \operatorname{dom}(\mathcal{A})|| x \mid=\varepsilon^{\prime}\right\}$ is nonempty. If there does not exist such $\varepsilon^{\prime}>0$, then $\operatorname{dom}(\mathcal{A})=\{0\}$, and nothing needs to be proven. Let $\mathcal{D}_{\varepsilon^{\prime}} \triangleq\left\{x \in \operatorname{dom}(\mathcal{A})|| x \mid \leq \varepsilon^{\prime}\right\}$. Let $\alpha \triangleq \min _{x \in \mathcal{B}_{\varepsilon^{\prime}}} V(x)$, then $\alpha>0$ due to positive definiteness of $V(\cdot)$ on $\operatorname{dom}(\mathcal{A})$. Take $\beta \in(0, \alpha)$ and let $\mathcal{E}_{\beta} \triangleq\left\{x \in \mathcal{D}_{\mathcal{\varepsilon}^{\prime}} \mid V(x) \leq \beta\right\}$. The set $\mathcal{E}_{\beta}$ is contained in $\mathcal{D}_{\mathcal{\varepsilon}^{\prime}}$. For every $x_{0} \in \mathcal{E}_{\beta}$, the corresponding solution $x\left(t ; x_{0}\right)$ stays in $\operatorname{dom}(\mathcal{A})$ and for that solution $\frac{d}{d t} V\left(x\left(t ; x_{0}\right)\right) \leq 0$. Hence, $x_{0} \in \mathcal{E}_{\beta}$ implies that $x\left(t ; x_{0}\right) \in \mathcal{E}_{\beta}$ for every $t \geq 0$. Take $\delta>0$ such that $\mathcal{D}_{\delta} \subset \mathcal{E}_{\beta} \subset \mathcal{D}_{\varepsilon^{\prime}}$, then we have shown that

$$
x_{0} \in \mathcal{D}_{\delta} \Rightarrow x_{0} \in \mathcal{E}_{\beta} \Rightarrow x\left(t ; x_{0}\right) \in \mathcal{E}_{\beta} \Rightarrow x\left(t ; x_{0}\right) \in \mathcal{D}_{\varepsilon^{\prime}},
$$

and hence $\{0\}$ is stable.

To show attractivity, note that $t \mapsto V\left(x\left(t ; x_{0}\right)\right)$ is monotonically decreasing and bounded from below, so there exists $a>0$ such that $V\left(x\left(t ; x_{0}\right)\right) \searrow a$ as $t \rightarrow \infty$. We need to show that $a=0$. Suppose not, then $V\left(x\left(t ; x_{0}\right)\right)>a$, for $x_{0} \in \mathcal{D}_{\delta}$. Choose $b>0$ such that $\mathcal{D}_{b} \subset \mathcal{E}_{a}$, which means $\left|x\left(t ; x_{0}\right)\right|>b$ for all $t \geq 0$. Let $c \triangleq \min _{b \leq|x| \leq \varepsilon^{\prime}} W(x)$, and because of assumptions on $W(\cdot)$, we have $c>0$. It follows from $(6.7)$ that $V\left(x\left(t ; x_{0}\right)\right) \leq V\left(x_{0}\right)-c t$, and hence for some $t$ large enough $V\left(x\left(t ; x_{0}\right)\right)<0$, which is a contradiction with $a>0$. To conclude, $V\left(x\left(t ; x_{0}\right)\right) \searrow 0$ and the asymptotic stability of the origin is established.

Let us use the result of Theorem 6.3 to recover a stability result for EVIs of the form:

$$
\langle\dot{x}-f(x), v-x\rangle+\varphi(v)-\varphi(x) \geq 0, \quad \forall v \in \mathbb{R}^{n}, \forall x \in \operatorname{dom}(\partial \varphi)
$$

where $\varphi: \mathbb{R}^{n} \rightarrow \mathbb{R} \cup\{\infty\}$ is convex proper LSC, and $f: \mathbb{R}^{n} \rightarrow \mathbb{R}^{n}$ is continuous hypomonotone. Such an EVI admits a unique right-differentiable absolutely continuous solution for each initial condition in $\operatorname{dom}(\partial \varphi)$. Using the definition of the subdifferential of $\varphi(\cdot)$, the inequality (6.8) is equivalently written as an inclusion

$$
\dot{x}-f(x) \in-\partial \varphi(x), \quad \forall x \in \operatorname{dom}(\partial \varphi) .
$$

By taking $\varphi(\cdot)$ to be the indicator function of a closed set, we recover the formalism of the FOSwP (with static set) from (6.8). The following proposition is adapted from the results given in [278, Lemma 3, Theorems 14, 15, 16], inspired from [283] (see also $[14,280,134]$ for similar results). 
Proposition 6.4. Consider the EVI (6.8) with $\operatorname{dom}(\partial \varphi)$ closed, $0 \in \operatorname{dom}(\partial \varphi)$, and $f(0) \in-\partial \varphi(0)$. Assume that there exists a continuously differentiable function $V(\cdot)$ such that $V(\cdot)$ is positive definite on $\operatorname{dom}(\partial \varphi)$, and

$$
\langle f(x), \nabla V(x)\rangle+\varphi(x-\nabla V(x))-\varphi(x) \leq-\lambda V(x), \quad \forall x \in \operatorname{dom}(\varphi) .
$$

If (6.10) holds with $\lambda=0$, then the origin $x^{\star}=0$ is Lyapunov stable. Moreover, if $V(x) \geq c\|x\|^{r}$ for $x \in \operatorname{dom}(\varphi)$ for some $c, r>0$ and (6.10) holds with $\lambda>0$, then $x^{\star}$ is globally exponentially stable.

The result is proved by showing that the condition (6.10) implies the inequality in (6.7). Indeed, for each $w \in-\partial \varphi(x)$, and $x \in \operatorname{dom}(\partial \varphi)$ we have

$$
\langle w, v-x\rangle+\varphi(v)-\varphi(x) \geq 0, \quad \forall v \in \mathbb{R}^{n} .
$$

By choosing $v=x-\nabla V(x)$, for each $x \in \operatorname{dom}(\partial \varphi)$, we get

$$
\max _{w \in-\partial \varphi(x)}\langle\nabla V(x), w\rangle \leq \varphi(x-\nabla V(x))-\varphi(x) .
$$

Substituting this bound in $\max _{w \in-\partial \varphi(x)}\langle\nabla V(x), f(x)+w\rangle$, leads to the inequality in (6.10), and the desired result follows from applying Theorem 6.3.

Finite-time stability. The condition (6.10) is slightly modified in [10, Theorems $3.5,3.6]$ to guarantee finite-time stability, where $\leq-\lambda V(x)$ is replaced by $\leq-g(V(x))$ with $\int_{0}^{\epsilon} \frac{d z}{g(z)}<+\infty$ for all $\epsilon>0$ (this kind of integral boundedness is classical to assure finite-time stability).

Constrained systems and copositive Lyapunov functions. When $\varphi(\cdot)=\psi_{\mathcal{S}}(\cdot)$, the indicator function of closed convex $\mathcal{S} \subset \mathbb{R}^{n}$, one obtains the DI: $\dot{x}-f(x) \in-\mathcal{N}_{\mathcal{S}}(x)$. The stability condition then boils down to $\langle\nabla V(x), f(x)\rangle \leq 0$ for all $x \in \mathcal{S}$, and $\left[\nabla V(x) \in-\mathcal{T}_{\mathcal{S}}(x)\right.$ for all $\left.x \in \operatorname{bd}(\mathcal{S})\right] \Leftrightarrow[x-\nabla V(x) \in \mathcal{S}$ for all $x \in \operatorname{bd}(\mathcal{S})]$. In particular, when $\mathcal{S}=\mathbb{R}_{+}^{n}$, the positive orthant, then we seek a Lyapunov functions $V$ which is positive definite on $\mathbb{R}_{+}^{n}$ and the inequality in (6.10) hold on $\mathbb{R}_{+}^{n}$. These conditions naturally yield copositive Lyapunov functions for systems with constrained state (LCS with relative degree one, EVIs as in (3.3)) [280]: the Lyapunov equation and matrix are required to be copositive on a closed convex set (see Definition B.2), rather than positive (semi) definite. Copositivity is also used in $[158,157]$ for relative degre zero LCS (essentially, with $D$ a P-matrix), using Lyapunov functions of both the state and the multiplier $\lambda$ in (2.22). Later copositivity has been used for switched positive systems in [288], and for conewise linear systems in [324], for LCS in [321] (the conditions stated in [324, 323, 321], basing on a characterization of copositive matrices taken from [239, Corollary 2.21] and [514, Theorem 2.1], being essentially the same as those in [280]).

Time-varying systems. Still dealing with systems possessing continuous solutions, let us cite the so-called Lyapunov pairs $(V(t, x), W(x))$ (whose definition is very close to dissipation inequality in (D.2) with null input) [62, Chapter 6] [513, Chapter 8] $[207,300,301,21,34,35,38,32,31,30,556,341,376]$, which are used to prove stability of equilibria, but also existence of solutions and invariance of sets in the FOSwP. The proximal normal cone and the proximal subdifferential (see Sections A.1.2 and A.2.1) play a particular role in the characterization and existence of Lyapunov pairs, where the Lyapunov functions are assumed to be LSC (hence possibly discontinuous). Their characterization is close in spirit to [70, Theorem 6.5] [69], however with non compact set-valued right-hand sides. Roughly speaking, the classical Lyapunov inequality $\frac{\partial V}{\partial t}+$ $\frac{\partial V}{\partial x} f(t, x) \leq-W(x)$ is substituted with $\theta+\xi^{T} f(t, x) \leq-W(x)$, where $\theta$ and $\xi$ are proximal subgradients (the inequality in (6.7) is an intermediate case). 
Other results and extensions. The stability of periodic trajectories of FOSwP is studied in [344]. Nonsmooth Lyapunov functions (often dealing with DIs as in (2.16) and incorporating $\mathcal{F}(\cdot)$ in the Lyapunov function, as a kind of pseudo-potential energy term), are used in $[21,253,218]$. The stability of PDS in (2.31) has been analysed in $[584,468,327,178,196]$, and for PDS as in $(2.32)$ or $(2.33)$ this is made in [291, 571], while quasi-stability is analysed in [183], see also [258]. An interesting feature pointed out in [584, Theorem 3.2], when $K$ is convex polyhedral, is that equilibria which are extreme points of $K$ are finite-time attractive (this does not seem to have been noticed anywhere else). This leads naturally to the consideration of the preservation of useful properties like finite-time or fixed-time Lyapunov stability, numerical chattering suppression (some kind of instability, well-known in sliding-mode control, but also present in the explicit discretization of maximal monotone DIs [477]), of discrete-time proximal algorithms to solve convex optimization problems (or VIs of first or second kinds). In [481], consistent discretizations are defined which are very close to backward Euler discretization in sliding-mode control $[5,320]$. Optimization problems could benefit from such studies, see [263] for first results in this direction. In all these works, the monotonicity of $f(\cdot)$ is shown to play a crucial role for Lyapunov stability. This is a feature that is shared with other stability results to be found in $[126,386,547,385]$, and which shows the proximity of our systems with the classical Lur'e systems (both blocks in Figure 1.1 should possess some kind of passivity property). The stability of fixed points in saddle-point dynamics (which belongs to maximal monotone DIs) is studied in [275], a converse Lyapunov theorem for LCS of relative degree zero can be found in [157, Theorem 5.2]. State observers design and stability analysis have been studied for passive DIs as (2.17) with AC solutions [135], LCS in (2.22) [306], DIs as (2.16) and FOSwP with $\mathrm{AC}$ and piecewise continuous solutions [138], impulsive observers [187]. Let us mention also time-delay in FOSwP [107] and in Lur'e systems $[318,245]$, using Lyapunov-Krasovskii functionals. Finally, necessary condition for Lyapunov stability in a class of maximal mononotone DIs are studied in [281].

6.2.2. Second order case. Lyapunov functions of both the state and the multiplier (the contact forces in Contact Mechanics, $\lambda$ in LCS (2.22)), have been used also for the stability analysis of mechanical systems with set-valued Coulomb's friction [76, 331, 330, 482]. This is related to so-called Hill's stability [331, Lemmae 8, 9] [330], borrowing ideas from [184]. Basically, this means that one does not consider perturbations solely on the state, but also perturbations on the multipliers (the contact forces), in order to analyse the stability. Attractivity, stability of sets and stability of equlibria in systems with Coulomb's friction (and known normal contact forces), have also been studied in [575, Lemma 1.6] [20, 13]. Notice that most of the above works use differentiable Lyapunov functions (an exception being the analysis of Lyapunov pairs for the FOSwP and the ZOSwP). Their extension towards LSC, right-Dini-differentiable functions $[70,513]$ could be interesting.

Let us summarize now contributions to the stability of nonsmooth mechanical systems:

- The Lejeune-Dirichlet (or Lagrange-Dirichlet) Theorem is extended to frictionless multicontact systems in $[131,126]$, and to the case with set-valued friction in $[386,385,547]$.

- Stability through finite accumulation of impacts (the so-called Zeno equilibria) is analysed in [453, 452, 383, 147, 568], with Lyapunov function [453, 383]. Notice however that accumulation points (Zeno points) are not necessarily equilibria [383].

- Stability analysis via the Zhuravlev-Ivanov nonsmooth change of state variable 
(see Section 3.14) and a Lipschitz continuous Lyapunov function, is tackled in $[459,458]$ (see also [130, Section 7.5.5]). It uses the twisting control algorithm [454].

- Synchronization of impacting systems is studied in [94, 95], using incremental stability and a specific distance function to cope with jump-time mismatch to design a Lyapunov function.

- The related topic of design and stability analysis of state observers has been analysed for MDIs like FOSwP with convex and prox-regular sets [522, 524] with $\mathrm{AC}$ or BV solutions (hence allowing for Zeno solutions), SOSwP in (4.10) [523], vibro-impact dynamics (i.e., no persistent contact phases) in $[252,262,411,417$, $419,418]$.

- Finite-time stability and convergence in continuous-time and in discrete-time systems [459, 458, 385, 547, 16, 146, 10, 255, 253, 423, 421, 422]: finite-time can be obtained by means of "plastic" impact (or accumulation of impact times), or using non-Lipschitz dynamics near the equilibrium (set-valued friction, slidingmode control).

- Stability of equilibria or periodic trajectories in vibro-impact systems using impact Poincaré maps [68, 380, 567] [130, Section 7.3].

- Convergence and its relation with monotonicity in MDIs [386, 385].

- Stability criteria taylored for generic robotic tasks, based on non-monotonic Lyapunov functions, are proposed in [118, 141, 142, 429, 428, 383], see [130, Definition 8.3, Propositions 8.1, 8.5]. They take into account possible changing dimensions (increasing or decreasing) in systems with multiple unilateral constraints and Zeno solutions for trajectory tracking [118, 142, 429, 428, 386], or equilibrium Lyapunov stability [383, Theorem 1]. In other words, these articles deal with the SOSwP in (2.6) (4.10), thus incorporating persistent contact phases. Local stability usually holds with co-existence of stable equilibrium and periodic trajectories [383].

REMARK 25. It is noteworthy that a complete stability analysis of the SOSwP (or of any system with $B V$ solutions), is based on the use of the differential measure $d V(\cdot)$, equivalently the density $\frac{d V}{d \mu}$ (see (5.22)). In particular this allows one to cope with the singular part of the derivative (in the sense of measures) of BV velocities. See [522, Remark 4.9]. This was advocated first in [126], then used in [522, 523, 386, 385].

6.3. Krasovskii-LaSalle invariance principle. The invariance principle, that is widely used in the literature on systems and control theory for asymptotic stabilization purpose, has been analyzed for our set-valued (autonomous) systems. For a generic statement of the invariance principle, we introduce the notion of limit set $\Lambda\left(x_{0}\right)$ associated with an initial condition $x_{0}$, which is defined as,

$$
\Lambda\left(x_{0}\right)=\left\{z \in \mathbb{R}^{n} \mid \exists\left\{\tau_{i}\right\} \subset[0,+\infty), \tau_{i} \rightarrow+\infty \text { and } x\left(\tau_{i} ; x_{0}\right) \rightarrow z\right\} .
$$

We recall that a set $\Psi \subset \mathbb{R}^{n}$ is positively invariant if $x(0)=x_{0} \in \Psi$ implies that the resulting solution $x\left(t ; x_{0}\right) \in \Psi$ for all $t \geq t_{0}$. The forward invariance property of the limit set $\Lambda\left(x_{0}\right)$ is useful in establishing the following result:

Theorem 6.5 (Invariance Principle). Consider the system (6.6), and assume that the following two properties hold:

(P1) There exists a positively invariant compact set $\Psi \subset \operatorname{dom}(\mathcal{A})$.

(P2) The limit set $\Lambda\left(x_{0}\right)$, for each $x_{0} \in \Psi$, is positively invariant. 
Suppose that there exists a continuously differentiable $V: \mathbb{R}^{n} \rightarrow \mathbb{R}$ satisfying

$$
\max _{\xi \in \mathcal{A}(x)}\langle\nabla V(x), \xi\rangle \leq-W(x), \quad \forall x \in \Psi
$$

for some continuous function $W: \mathbb{R}^{n} \rightarrow \mathbb{R}_{+}$. Consider the set $\mathcal{Z} \triangleq\{x \in \Psi \mid W(x)=$ $0\}$, and let $\mathcal{P}$ be the largest invariant set contained in $\mathcal{Z}$. Then, it holds that, for each $x_{0} \in \Psi$,

$$
\lim _{\tau \rightarrow+\infty} d_{\mathcal{P}}\left(x\left(\tau ; x_{0}\right)\right)=0
$$

Proof. For a fixed $x_{0} \in \Psi$, let $x\left(t ; x_{0}\right)$ be a corresponding solution which stays in

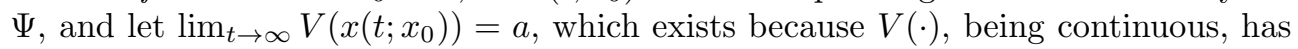
a well-defined minimum value on the compact set $\Psi$. Boundedness of $\Psi$ implies that $\Lambda\left(x_{0}\right) \neq \emptyset$, and $\Psi$ being closed implies that $\Lambda\left(x_{0}\right) \subset \Psi$. Choose a point $z \in \Lambda\left(x_{0}\right)$, then by definition, there exists an unbounded increasing sequence $\left\{\tau_{j}\right\}_{j \in \mathbb{N}}$ such that $\lim _{j \rightarrow \infty} x\left(t_{j} ; x_{0}\right)=z$, where $x\left(t_{j}\right) \in \Psi$, for each $j \in \mathbb{N}$. The continuity of $V(\cdot)$ gives that $V(z)=\lim _{j \rightarrow \infty} V\left(x\left(t_{j} ; x_{0}\right)\right)=a$ for each $z \in \Lambda\left(x_{0}\right)$. Forward invariance of $\Lambda\left(x_{0}\right)$ implies that, for each $z \in \Lambda\left(x_{0}\right)$, we have $z \in\{x \in \Psi \mid \dot{V}(x)=0\} \subset \mathcal{Z}$. Since $\Lambda\left(x_{0}\right)$ is positively invariant, $\Lambda\left(x_{0}\right) \subset \mathcal{P} \subset \mathcal{Z}$. Also, with $\Psi$ being bounded, for every $x_{0} \in \Psi$, we obtain $\lim _{t \rightarrow \infty} x\left(t ; x_{0}\right) \in \Lambda\left(x_{0}\right)$, which proves the desired assertion.

6.3.1. Specific Cases. Note that Theorem 6.5 does not require $V(\cdot)$ to be positive definite on $\operatorname{dom}(\mathcal{A})$. However, to establish the property $(\mathrm{P} 1)$ in Theorem 6.5, one can work with a positive definite function $V(\cdot)$ that satisfies $\max _{\xi \in \mathcal{A}(x)}\langle\nabla V(x), \xi\rangle \leq$ $-W(x)$ for some continuous $W: \mathbb{R} \rightarrow \mathbb{R}_{+}$. In this case, there exists some $c>0$, $\{x \in \operatorname{dom}(\mathcal{A}) \mid V(x) \leq c\}$ is compact and positively invariant.

The other key property in the invariance principle, i.e. (P2), is guaranteed by the continuity of solutions with respect to the initial conditions (other conditions exist like the pre-compactness of solutions [495, Proposition 2.8, Theorem 2.10]). In particular, we have the following result from [494], [385, Prop. 6.12]:

Proposition 6.6. Consider the system (6.6) and let $\Lambda\left(x_{0}\right)$ be defined as in (6.11). If, for every $x_{0} \in \operatorname{dom}(\mathcal{A})$ and every $t \geq t_{0}$, the mapping $x_{0} \mapsto x\left(\cdot ; x_{0}\right)$ is uniformly continuous, then $\Lambda\left(x_{0}\right)$ is forward invariant.

In most first order nonsmooth systems, we have the continuity of solutions with respect to initial conditions under mild conditions on system's data. However, SOSwP (with non orthogonal unilateral constraints) do not share this property, see Section 5.4.2. See also [385, p.146-147] for a simple case of a PWL system whose limit sets are not invariant. For the EVI (6.8), the aforementioned continuity indeed holds and this allows us to state the invariance principle for EVI (6.8) as a special case of Theorem 6.5.

Theorem 6.7 (Invariance Theorem for EVIs). Consider the EVI (6.8) with $\operatorname{dom}(\partial \varphi)$ closed, $0 \in \operatorname{dom}(\partial \varphi)$, and $f(0) \in-\partial \varphi(0)$. Let $\Psi \subset \operatorname{dom}(\partial \varphi)$ be a compact set, and suppose that there exists $V: \mathbb{R}^{n} \rightarrow \mathbb{R}_{+}$continuously differentiable, such that

$$
\langle f(x), \nabla V(x)\rangle+\varphi(x-\nabla V(x))-\varphi(x) \leq-W(x), \quad x \in \Psi,
$$

for some continuous $W: \mathbb{R}^{n} \rightarrow \mathbb{R}_{+}$. Let $\mathcal{Z} \triangleq\{x \in \operatorname{dom}(\varphi) \mid\langle f(x), \nabla V(x)\rangle+\varphi(x-$ $\nabla V(x))-\varphi(x)=0\}$. Let $\mathcal{P}$ be the largest invariant subset of $\mathcal{Z} \cap \Psi$. Then, for each initial condition $x_{0}=x(0) \in \operatorname{dom}(\partial \varphi)$ such that $\left\{x\left(\tau ; x_{0}\right) ; \tau \geq t_{0}\right\} \subset \Psi$, it holds that $\lim _{\tau \rightarrow+\infty} d_{\mathcal{P}}\left(x\left(\tau ; x_{0}\right)\right)=0$. 
This result somewhat similar to the results appearing in [134, 278]. An extension of Theorem 6.7 could consider set-valued mappings $f(\cdot)$.

6.3.2. Other Results. Some of the contributions in developing the invariance principle use the continuity in the initial data property [278, Section 5.4] [14, Sections 3.3,5.3.2] [20, Theorem 2, Section 3] [13, Theorems 3.3,3.4] [28, Theorems 6,7] [281, Theorem 3] [50, 279, 303] [134, Theorems 2,4,5, Corollaries 1,2,3,4] [135, Section 5] [157, Theorem 3.1, Proposition 3.2]. Other articles do not, but directly use the positive invariance of limit sets [36, Theorems 6,7, Corollaries 2,3] [17, Lemma 6.5, Theorem 6.6] [385, Theorems 6.31, 7.6] [108, Proposition 1] (relying on [495]). This property holds generically for DIs with compact, convex valued and upper semicontinuous right-hand sides [249], and we note that the DIs considered in [495] satisfy these assumptions. The stability analysis in [131] uses the results in [385].

It is noteworthy that all these results use a single Lyapunov-like function, however not necessarily $\mathcal{C}^{1}$ (it is Lipschitz continuous in [50, 455], discontinuous LSC in $[108,495])$. Krasovskii-LaSalle's invariance principle has been shown to apply to autonomous systems with: (i) continuous solutions: LCS with relative degree zero ( $D$ is a P-matrix) [157], DIs as in $(2.17)$ with passive $(A, B, C, D)$ (see Definition D.1) [135, 28], evolution VIs of the first kind [134], and of the second kind $[278,14,13,20,281]$ (the dynamics in [108, equ. (10)] can be recast into these formalisms), PDS [303], Lagrangian systems with maximal monotone right-hand side in $\dot{q}[17,20]$, FOSwP with prox-regular $\mathcal{S}(t)=\mathcal{S}$ and Lipschitz continuous perturbation (using for instance [33, Theorem 4.2, Corollary 4.1] which recasts such FOSwP into DIs with maximal monotone right-hand side), and (ii) to SOSwP with BV solutions [385]. Weakly invariant sets $\Lambda\left(x_{0}\right)$ can be used [36] (a crucial property is then that the DI right-hand side be upper semicontinuous [249, p. 129]). It is noteworthy that the criteria in Proposition 6.4 and Theorem 6.7 are not well suited to mechanical systems with Coulomb's friction (which nevertheless fit within the considered class of EVI). The material in [20] applies to such systems, see also [108] where the authors use a discontinuous LSC Lyapunov function, and [547] for local attractivity of equilibria sets with $V(\cdot)$ the total mechanical energy.

REMARK 26. Surprizingly enough, an issue which remains open decades after Krasovskii and LaSalle's publications, is: given any dynamical system that enjoys positive invariance of its limit sets, what are the conditions on the solution set and on the Lyapunov function regularity, so that the invariance principle applies automatically? Preliminary answer is given in [455, Theorem 3.2] for a class of systems with discontinuous right-hand side, possessing solutions uniquely continuable to the right, and relying on earlier results in [312, Section 4.3] formulated for general dynamical systems defined on complete metric spaces. Let us note the application of the discretetime invariance principle with Poincaré impact maps in [380, Proposition 8]. An open issue is to extend the LaSalle-Yoshizawa Theorem to our DIs with time-varying terms (including the FOSwP), which is known to apply to Filippov's DIs [250].

6.4. Passivity and absolute stability. Omnis honor omni domino praestandum est (Pay honour to whom honour is due). This article began with the contribution of J.J. Moreau, the pioneer of nonsmooth mechanics and one of the founders of convex analysis. Let us start this last section with the very first contribution on absolute stability for set-valued systems, due to Vladimir Yakubovich, one of the primary contributors of the celebrated Kalman-Yakubovich-Popov Lemma [139, Chapter 3], a cornerstone of Automatic Control. In [573], he considered positive real systems with hysteresis feedback satisfying a sector nonlinearity, and derived various stability 
results with frequency conditions; see also [139, Chapter 2] for recent exposition.

Indeed, passivity is a major tool in systems and control theory [139]. It is closely related to the absolute stability problem, which consists of studying the Lyapunov stability of the negative feedback interconnection in Figure 1.1, with a passive upperblock and a feedback nonlinearity that satisfies a sector condition. Passivity is used to show the well-posedness and the stability of LCS $(2.22)$ in $[151,157,155]$, of LCS with time-delay [107], and of PWL systems which can under some conditions be interpreted in our framework (see Section 3.11) [533]. These results are based on the LMI in (D.1) and (D.3). The Lyapunov stability of DIs as (2.16) with $f(t, x)=-\nabla V(x)$ and $\mathcal{F}(t, x)=\mathcal{N}_{\mathcal{S}}(x), \mathcal{S}$ prox-regular, and with continuously differentiable Lyapunov functions $V(\cdot)$, is analysed in [374, Proposition 3.1] where a dissipation equality is shown. The complete dissipation equality for a class of subdifferential DIs with $\mathcal{F}(x)=\partial \varphi(x), \varphi(\cdot)$ convex LSC, and $f(t, x)$ set-valued with compact convex values, is shown in [100, Theorem 2.7]. Passivity in DIs like (2.17) is used in $[135,159,29]$. Passivity of $(A, B, C, D)$ in $(2.17)$ is used in [135, Theorem 5] to state a simplified version of Theorem 6.7 as follows.

TheOREM 6.8 (Invariance principle for passive systems [135]). Consider the DI in (2.17) with $\mathcal{M}(z)=\partial \varphi(z), \varphi(\cdot)$ proper convex $L S C$, and $(A, B, C, D)$ passive with LMI solution $P=P^{T} \succ 0$. Assume that $\varphi^{*}(-z)>\varphi^{*}(0)$ for all $z \neq 0$. Let $\mathcal{P}$ be the largest invariant subset of $\mathcal{Z}=\left\{z \in \mathbb{R}^{n} \mid z^{\top}\left(A^{\top} P+P A\right) z=0\right\}$. Then for each $x_{0} \in \operatorname{dom}(\mathcal{M})$, one has $\lim _{t \rightarrow+\infty} d_{\mathcal{P}}\left(x\left(t ; x_{0}\right)\right)=0$.

Applying this result to the mechanical system in Example 3 requires a modification allowing for $\varphi^{*}(-z) \geq \varphi^{*}(0)$. The main difference between Theorem 6.8 and Theorem 6.7 , is that passivity implies the existence of a quadratic positive definite Lyapunov function, so that trajectories and their orbits are bounded (the set $\Psi$ of Theorem 6.7 need not be considered), and $\Lambda\left(x_{0}\right) \neq \emptyset$. However, the condition $\varphi^{*}(-z)>\varphi^{*}(0)$ is somewhat restrictive: on one hand it allows to look for the attractive set in terms of matrices $A$ and $P$, but on the other hand it imposes a strong requirement on the nonsmooth term $\lambda \in \mathcal{M}(z)$, that is, $\lambda=0$ for each $z \neq 0$. A more careful study is therefore required to derive a more generic invariance principle in the context of maximal monotone differential inclusions under passivity assumption. It is also likely that [385, Theorems 6.31, 7.6] and [130, Lemma 7.1] could be merged and generalized to extend Theorem 6.8 to Lagrangian systems with unilateral constraints, Coulomb friction and impacts (see [131, Proposition 2] for the case of frictionless multibody systems with joint clearance).

It is noteworthy that the input/output constraint $P B=C^{T}, P=P^{T} \succ 0$ (see Section 3.4) which is implied by (D.1) when $D+D^{T}=0$, and its generalization $\operatorname{ker}\left(D+D^{T}\right) \subset \operatorname{ker}\left(P B-C^{T}\right)$ used in Corollary 5.9 (see [139, Propositions 3.62, 3.63] $[151,155]$ for structural properties of dissipative systems), are passivity-like conditions stemming from the celebrated Kalman-Yakubovich-Popov (KYP) Lemma [139, 155]. It follows that the results in $[9,11,12,20,28,36,31,39,134,135,136,138,143$, $280,386,522,524]$, extended in [523, 159] and in [143, Section 4], rely on a sort of passivity condition on the continuous-time system, not only for stability but also for well-posedness purposes. One consequence is that state jumps (according to the jump mappings in Section 2.4.4) are easily incorporated in the stability analysis because the storage function of Definition 2.2 is a good Lyapunov function candidate.

Passivity conditions are also used for the semi-global stability of FOSwP with proxregular sets $\mathcal{S}(t)$ is analysed in [522, Theorem 3.2, Proposition 3.5], using $r$ from Definition A.2 as a parameter to determine the size of the basin of attraction (the 
stability of the convex case being recovered as $r \rightarrow+\infty$ ). This seems to be the first result of this type in the literature, a partial extension of [522, Theorem 3.2] is in [33, Theorem 6.1, Corollary 6.1]. The result in [522, Theorem 3.2] may be considered as the first version of absolute stability with a nonconvex set-valued feedback nonlinearity. See [562] for another analysis. KYP Lemma LMIs are used in [245] to show absolute stability of singular DIs as in (2.18). Passivity is used in [73] for the well-posedness analysis of (4.9), and in the HOSwP for stability purpose [6]. The extension of the dissipation inequality (D.2), to SOSwP, has been proposed in $[147,130][139$, Section 7.2.4.1], incorporating state jumps through a suitable definition of the supply rate and the use of differential measures of BV functions. The closed-loop systems in [421, Equ. (8) (9) (11)] [423, Equ. (3) (4)] also fit within (1.1) with a passive subsystem (the Lagrange dynamics, with a perturbation) in feedback with a maximal monotone static feedback law (a sliding-mode controller), and can be considered as nonlinear set-valued Lur'e systems, see also [523, Section VI.C] for state observer dynamics in Lagrangian systems.

The classical absolute stability problem, which has been widely studied in the control systems community, can be addressed via the circle and the Popov criteria. Generalisations of the circle criterion in the set-valued case are presented in [575], mainly focusing on signum feedback nonlinearities, and on piece-wise differentiable, maximal monotone nonlinearities $\mathbb{R} \rightarrow \mathbb{R}[575$, Theorem 3.10], where maximality is obtained by filling-in the graphs (the class of linear invariant systems in [575, Theorem 3.10] is less restrictive than [126] [135, Theorem 3] [139, Lemmae 3.125, 3.129], but the considered class of static feedback nonlinearities is much less general). The circle criterion for Lur'e set-valued systems with $\mathcal{F}(x)$ compact convex upper semicontinuous is presented in [334], the Popov criterion for maximal monotone DIs is analysed in [215], the absolute stability problem with positive real frequency conditions is studied in [387, 264]. Multipliers techniques to transform feedback systems is also a mature field of control systems, for regular ODEs. The use of O'Shea-Zames-Falb multipliers for set-valued Lur'e systems is advocated in [500] to reduce conservativeness of the results in [126]. Systems where the feedback loop $\mathcal{F}(\cdot)$ is a hysteresis nonlinearity are tackled in $[333,573,80,359]$ within the framework of Lur'e set-valued systems.

7. Conclusions. Inspired by the structure of Lur'e systems and the tools used in their analysis, this article addressed a class of differential inclusions where the righthand side is the sum of a single-valued mapping and a set-valued mapping with some monotone properties. The motivation for studying such dynamical systems arises from various physical systems, and optimization algorithms. Five main fields are covered: a brief description of the mathematical formalisms, the relationships between them, the various fields of application, well-posedness (existence, uniqueness, continuous dependence of solutions), and stability issues. Bifurcation and chaos analysis is not treated, since it is largely covered elsewhere [384, 224]. By citing several references on each of these topics, we have provided an overview of the progress that has been made in the analysis of such systems. It has been interesting to see that, while initially mainly used in mechanics or electronics, the models studied in this paper have made their way across several engineering disciplines. We have identified some directions of research that could contribute to advancement of this field, and here we summarize them with concluding remarks.

Formalisms: We considered several mathematical models which could be rewritten in the form of (1.1). A key component of these models is that the nonsmoothness entering in the dynamics is obtained from a solution to an optimization problem (such 
as quadratic program with linear constraints for an LCS). Some works exist in the literature to draw connections between formalisms [133, 143, 229, 266, 308, 462] and here we have reviewed them with some additional insights. However, a better understanding is required for connecting different models and exploiting the structure of optimization problems. A formal treatment of such connections can also pave way for borrowing analysis techniques developed for one system class to another. In the process, one can better study the questions of existence of solutions.

Numerics: The time-discretization problem is addressed only for the existence of solutions, see $[2,4]$ for a complete presentation of numerical analysis issues and simulation algorithms. The numerical algorithms used for simulation of these systems typically use time-discretization schemes as well. However, there have been relatively very few works which rigorously analyze the performance of these numerical algorithms. Most notably, the order of convergence for several classes of numerical algorithms have not been analyzed. The accuracy of the solutions in case of numerical errors (in computing the projection on to a set for example) has not been much investigated.

Stability and Control: We investigated the stability for a certain class of nonsmooth systems, but mostly our investigation was limited to first order systems. Stability analysis for second order systems with impacts and friction is crucial for many applications (this is a field that may grow in importance in Robotics in the future years), but there are relatively fewer works in this direction. Feedback control and its many branches (optimal control, controllability, observability, state observer design and separation principle, stabilization, tracking control, classification of systems into subclasses depending on their controllability/observability properties, robust control) has not been treated in detail. While these problems have received some attention in the community $[287,309,526,555]$, there are many related questions which need to be investigated in depth, as they lead to some challenging questions at the intersection of functional analysis, optimization algorithms, numerical implementation, and control theory.

\section{Appendices}

A. Some tools from convex analysis. The material that follows is taken from diverse sources [240, 314, 97, 163, 191, 416, 143, 133, 62, 63, 41, 102, 279, 431, 282]. Since tangent and normal cones are central tools, we start with a brief exposition on their definitions, relationships and properties. Let $f: \mathbb{R}^{n} \rightarrow \mathbb{R}^{m}$ be differentiable, then its gradient at $x$ is denoted as $\nabla f(x) \in \mathbb{R}^{n \times m}$ and is the transpose of its Jacobian $\frac{\partial f}{\partial x}(x) \in \mathbb{R}^{m \times n}$.

A.1. Tangent and normal cones. Let $K \subseteq \mathbb{R}^{n}$ be a closed nonempty set, not necessarily convex. The polar cone of $K$ is $K^{\circ}=\left\{x \in \mathbb{R}^{n} \mid\langle x, y\rangle \leq 0\right.$ for all $\left.y \in K\right\}$. The dual cone of $K$ is $K^{\star}=-K^{\circ}$. If $K$ is a nonempty convex cone, then $\left(K^{\circ}\right)^{\circ}=K$. If $K$ is a linear subspace then $K^{\circ}=K^{\perp}=\left\{x \in \mathbb{R}^{n} \mid\langle x, y\rangle=0\right.$ for all $\left.y \in K\right\}$. Let us now make a brief review of normal and tangent cones, which are central tools for the dynamical systems dealt with in this work, and generalize the notions of tangent and normal subspaces. It happens that there are many different types of normal and tangent cones, which are not equal in general [63, Fig. 4.4]. Our aim in this appendix is to introduce few of them, which are most often met in the literature and are useful 
in practice (see [63] for a complete exposition).

A.1.1. Tangent cones. Let us start with tangent cones. For each $x \in K$ let us define $[62,416]$ :

1. Radial cone: $\mathcal{R}_{K}(x)=\left\{z \in \mathbb{R}^{n} \mid \exists t_{0}>0\right.$ such that $\left.x+t z \in K, \forall t \in\left(0, t_{0}\right)\right\}$.

2. Bouligand or Bouligand-Peano or contingent cone: $\mathcal{T}_{K}(x)=\left\{z \in \mathbb{R}^{n} \mid \exists\left\{t_{n}\right\}\right.$ $\subset \mathbb{R}_{+}, \exists\left\{z_{n}\right\} \subset \mathbb{R}^{n}$, such that $\left.t_{n} \searrow 0, z_{n} \rightarrow z, x+t_{n} z_{n} \in K, \forall n \in \mathbb{N}\right\}=\{z \in$ $\mathbb{R}^{n} \mid \exists\left\{x_{n}\right\} \subset K, \exists\left\{\lambda_{n}\right\} \subset \mathbb{R}_{+}, \lambda_{n} \searrow 0$, such that $x_{n} \rightarrow x$ and $\left.\frac{x_{n}-x}{\lambda_{n}} \rightarrow z\right\}$.

3. Adjacent tangent cone: $\mathcal{T}_{K}^{b}(x)=\left\{z \in \mathbb{R}^{n} \mid \forall\left\{t_{n}\right\} \subset \mathbb{R}_{+}\right.$such that $t_{n} \searrow$ $0, \exists\left\{z_{n}\right\} \in \mathbb{R}^{n}$ with $\left.z_{n} \rightarrow z, x+t_{n} z_{n} \in K, \forall n \in \mathbb{N}\right\}$.

4. Clarke tangent cone: $\mathcal{T}_{K}^{C}(x)=\left\{z \in \mathbb{R}^{n} \mid \forall\left\{x_{n}\right\} \subset K, \forall\left\{t_{n}\right\} \subset \mathbb{R}_{+}\right.$such that $x_{n} \rightarrow x, t_{n} \searrow 0, \exists\left\{z_{n}\right\} \in \mathbb{R}^{n}$ with $\left.z_{n} \rightarrow z, x_{n}+t_{n} z_{n} \in K, \forall n \in \mathbb{N}\right\}$.

Some properties of these tangent cones are as follows $[62,63,97,191,416]: \mathcal{T}_{K}(x)$, $\mathcal{T}_{K}^{b}(x), \mathcal{T}_{K}^{C}(x)$ are closed, $\mathcal{T}_{K}^{C}(x)$ is convex (even if $K$ is not convex), while the other cones may not be, $\mathcal{T}_{K}^{C}(x) \subset \mathcal{T}_{K}^{b}(x) \subset \mathcal{T}_{K}(x)$ (hence if $x \in \operatorname{int}(K)$, these three cones are equal to $\mathbb{R}^{n}$ ), and $\mathcal{R}_{K}(x) \subset \mathcal{T}_{K}^{b}(x)$. A so-called weak tangent cone $\mathcal{T}_{K}^{w}(x)$ can also be defined. However in case the space is finite dimensional (which is our case in general in this article) then $\mathcal{T}_{K}^{w}(x)=\mathcal{T}_{K}(x)$. When $\mathcal{T}_{K}^{C}(x)=\mathcal{T}_{K}(x), K$ is said tangentially regular at $x$ (this prevents for instance the existence of re-entrant corners in $K)$. Let $S_{K}(x)=\cup_{h>0} \frac{K-x}{h}$ be the cone spanned by $K-x$. Then $\mathcal{T}_{K}(x) \subset \overline{S_{K}(x)}$. If $K$ is closed nonempty convex, then all the above tangent cones (but the radial cone) are equal to $\overline{S_{K}(x)}$, with

$$
\mathcal{T}_{K}(x)=\operatorname{cl}\left(\left\{z \in \mathbb{R}^{n} \mid z=\lambda(y-x), \lambda \geq 0, y \in K\right\}\right),
$$

and $\operatorname{cl}\left(\mathcal{R}_{K}(x)\right)=\mathcal{T}_{K}(x)$.

A.1.2. Normal cones. Let us now introduce normal cones:

1. Clarke: $\mathcal{N}_{K}^{C}(x)=\left(\mathcal{T}_{K}^{C}(x)\right)^{\circ}=\left\{z \in \mathbb{R}^{n} \mid\langle z, y\rangle \leq 0\right.$ for all $\left.y \in \mathcal{T}_{K}^{C}(x)\right\}$.

2. Fréchet: $\hat{\mathcal{N}}_{K}(x)=\left(\mathcal{T}_{K}(x)\right)^{\circ}=\left\{z \in \mathbb{R}^{n} \mid \lim \sup _{y \rightarrow x, y \in K} \frac{\langle z, y-x\rangle}{\|y-x\|} \leq 0\right\}$.

3. Strong or norm limiting (or limiting or basic or Mordukhovich): $\mathcal{N}_{K}^{s}(x)=$ $\left\{z \in \mathbb{R}^{n} \mid \exists\left\{x_{n}\right\} \subset K, \exists\left\{z_{n}\right\} \in \mathbb{R}^{n}\right.$, with $x_{n} \rightarrow x, z_{n} \rightarrow z, z_{n} \in \widehat{\mathcal{N}}_{K}\left(x_{n}\right), \forall n \in$ $\mathbb{N}\}$.

4. Proximal: $\mathcal{N}_{K}^{p}(x)=\left\{z \in \mathbb{R}^{n} \mid\langle z, y-x\rangle \leq \sigma\|y-x\|^{2}\right.$ for some $\sigma \in \mathbb{R}_{+}, \forall y \in$ $K\}$.

Some properties of these normal cones are as follows $[191,416,431]: \mathcal{N}_{K}^{p}(x) \subset$ $\widehat{\mathcal{N}}_{K}(x) \subset \mathcal{N}_{K}^{s}(x) \subset \mathcal{N}_{K}^{C}(x)$, and $\mathcal{N}_{K}^{C}(x)=\{0\}$ if $x \in \operatorname{int}(K)$. The Mordukhovich normal cone $\mathcal{N}_{K}^{s}(x)$ never reduces to $\{0\}$ when $x \in \operatorname{bd}(K)$, contrarily to $\widehat{\mathcal{N}}_{K}(x)$. In our finite-dimensional case $\widehat{\mathcal{N}}_{K}(x)=\left(\mathcal{T}_{K}(x)\right)^{\circ}$, and $\mathcal{T}_{K}(x) \subset\left(\mathcal{N}_{K}^{p}(x)\right)^{\circ}$. Note that in infinite dimension, limiting -or basic, Mordukhovich- normal cones $\mathcal{N}_{K}(x)$, and strong limiting normal cones differ, with $\mathcal{N}_{K}^{s}(x) \subset \mathcal{N}_{K}(x) \subset \mathcal{N}_{K}^{C}(x)$. Also $\mathcal{N}_{K}^{C}(x)=$ $\overline{\operatorname{conv}}\left(\mathcal{N}_{K}^{s}(x)\right)$, thus it is closed convex even if $K$ is not convex, and $\mathcal{T}_{K}^{C}(x)=\left(\mathcal{N}_{K}^{C}(x)\right)^{\circ}$. However $\mathcal{N}_{K}^{s}(x)$ is not necessarily convex, see [431, Example 1.5] and the set $K_{6}$ in Example 10 below. When $\widehat{\mathcal{N}}_{K}(x)=\mathcal{N}_{K}^{s}(x), K$ is called normally regular (then $\mathcal{N}_{K}^{s}(x)=\mathcal{N}_{K}^{C}(x)$ as well), and Fréchet normally regular when $\widehat{\mathcal{N}}_{K}(x)=\mathcal{N}_{K}^{C}(x)$ (see [301, Proposition 1] [143, Equation (38)] for characterizations of normal regularity). Hence in finite dimension, normally regular, Fréchet normally regular, and tangentially regular are equivalent properties. When $K$ is $r$-prox regular (see Definition A.2), then $\mathcal{N}_{K}^{p}(x)=\widehat{\mathcal{N}}_{K}(x)=\mathcal{N}_{K}^{s}(x)=\mathcal{N}_{K}^{C}(x)$ [143] (but normal regularity and $r$-prox 
regularity are different notions), and $\mathcal{T}_{K}(x)=\mathcal{T}_{K}^{C}(x)$. In case $K$ is closed nonempty convex, then all the above cones are equal, and for all $x \in K$ :

$$
\mathcal{N}_{K}(x)=\left\{z \in \mathbb{R}^{n} \mid\langle z, y-x\rangle \leq 0, \quad \forall y \in K\right\} .
$$

It is noteworthy that the normal cone is sometimes defined like (A.2), for closed non convex sets [240, Equation (1.1.2)], though it is noticed in [240, Remark 1.3.3] that (A.2) is taylored to convex sets. It follows from (A.2) that $\mathcal{N}_{\{a\}}(x)=\mathcal{N}_{\{a\}}(a)=\mathbb{R}^{n}$ for any $a \in \mathbb{R}^{n}$, and $\mathcal{N}_{\mathbb{R}^{n}}(x)=\{0\}$. Another characterization for closed convex cones is $\mathcal{N}_{K}(x)=K^{\circ} \cap x^{\perp}$ where $x^{\perp}$ is the subspace orthogonal to $x$.

REMARK 27. When $x \notin K$, one usually sets all cones equal to $\emptyset$. There may be exceptions when one needs to work outside $K$ like in discrete-time approximations.

EXAMPLE 10. Some illustrating examples in finite dimension:

1. $K_{1}=\mathbb{R}^{n}: \mathcal{T}_{K_{1}}(x)=\mathbb{R}^{n}, \mathcal{N}_{K_{1}}(x)=\{0\}$.

2. $K_{2}=\left\{x_{0}\right\}$ for some $x_{0} \in \mathbb{R}^{n}: \mathcal{T}_{K_{2}}\left(x_{0}\right)=\{0\}, \mathcal{N}_{K_{2}}\left(x_{0}\right)=\mathbb{R}^{n}$.

3. $K_{3}=\left\{(x, y) \in \mathbb{R}^{2} \mid[y=0\right.$ and $x \geq 0] \cup[x=0$ and $\left.y \geq 0]\right\}=\operatorname{Graph}\left(-N_{\mathbb{R}_{+}}\right)$: at the origin we have $\mathcal{T}_{K_{3}}^{C}(0,0)=\{0\}, \mathcal{N}_{K_{3}}^{C}(0,0)=\mathbb{R}^{n}, \widehat{\mathcal{N}}_{K_{3}}(0,0)=\{(x, y) \in$ $\left.\mathbb{R}^{2} \mid x \leq 0, y \leq 0\right\}, \mathcal{T}_{K_{3}}(0,0)=K_{3}, \mathcal{N}_{K_{3}}^{s}(0,0)=\widehat{\mathcal{N}}_{K_{3}}(0,0) \cup K_{3}$.

4. $K_{4}=\left\{(x, y) \in \mathbb{R}^{2}|| x|=| y \mid\right\}$ : at the origin we have $\mathcal{T}_{K_{4}}(0)=\mathcal{T}_{K_{4}}^{b}(0)=K_{4}$, $\mathcal{T}_{K_{4}}^{C}(0)=\{0\}, \mathcal{N}_{K_{4}}^{C}(0)=\mathbb{R}^{2}, \widehat{\mathcal{N}}_{K_{4}}(0)=K_{4}^{\circ}$.

5. $K_{5}=\left\{(x, y) \in \mathbb{R}^{2} \mid x+y=1, x \geq 0, y \geq 0\right\}$ : at the two edges of the segment we have $\mathcal{T}_{K_{5}}((1,0))=\left\{\left(z_{1}, z_{2}\right) \mid z_{1}=-z_{2}, z_{2} \geq 0\right\}, \mathcal{T}_{K_{5}}((0,1))=\left\{\left(z_{1}, z_{2}\right) \mid z_{2}=\right.$ $\left.-z_{1}, z_{1} \geq 0\right\}$.

6. $K_{6}$ as depicted in Figure A.1 (b) has a reentrant corner (notice that we depict the sets $x+\mathcal{N}_{K}(x)$ for convenience). This set is neither normally nor tangentially regular at $x$. One has $\mathcal{N}_{K_{6}}^{s}(x)=S_{1} \cup S_{2}$, where $S_{i}, i=1,2$, is the half-line generated by $\mathbf{n}_{i}$ from $x$, and is thus non convex. And $\widehat{\mathcal{N}}_{K_{6}}(x)=$ $\mathcal{N}_{K_{6}}^{p}(x)=\{0\}$.

7. $K_{7}=\left\{x \in \mathbb{R}^{n} \mid k_{i}(x)=0,1 \leq i \leq m\right\}$, where each $k_{i}: \mathbb{R}^{n} \rightarrow \mathbb{R}$ is $C^{2}$ : If the gradients $\nabla k_{i}(x)$ are linearly independent, then $\mathcal{N}_{K_{7}}^{C}(x)=\mathcal{N}_{K_{7}}^{p}(x)=$ $\operatorname{span}\left(\nabla k_{i}(x)\right), \mathcal{T}_{K_{7}}^{C}(x)=\left\{v \in \mathbb{R}^{n} \mid \nabla k_{i}^{T}(x) v=0,1 \leq i \leq m\right\}$.

8. $K_{8}$ is depicted in Figure A.2 (a), it has an infinitely sharp symmetric reentrant corner at $x$. One has $\mathcal{N}_{K_{8}}^{s}(x)=S_{1} \cup S_{2}, \mathcal{T}_{K_{8}}(x)=\mathbb{R}^{2}$.

9. $K_{9}$ is depicted in Figure A.2 (b), it has an infinitely sharp "peak" at $x$, it is non convex and non prox-regular. One has $\mathcal{N}_{K_{8}}^{s}(x)=S_{1} \cup S_{2}$.

It is a fact that in general, normal cones may not be easy to compute, see, e.g., [267, Examples 2, 3].

A.1.3. Linearization cones. There are two other cones which are of high interest: the linearization cones when $K$ is finitely represented, which make a very important class of sets in practice (the above definitions being stated for general and abstract sets $K$, including infinite dimensional cases). Let $K=\left\{x \in \mathbb{R}^{n} \mid k(x) \geq 0\right\}$, where $k: \mathbb{R}^{n} \rightarrow \mathbb{R}^{m}$ is continuously differentiable, $K$ is not necessarily convex, and $\mathcal{I}(x)=\left\{i \in\{1, m\} \mid k_{i}(x)=0\right\}$ is the index set of active constraints. The linearized cones are defined as follows [133]:

1. Tangent cone linearization cone:

$$
\mathcal{T}_{K}^{h}(x)=\left\{z \in \mathbb{R}^{n} \mid z^{T} \nabla k_{i}(x) \geq 0, \text { for all } i \in \mathcal{I}(x)\right\} .
$$

2. Normal cone linearization cone: 


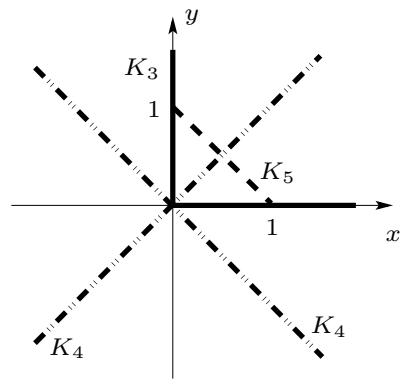

(a)

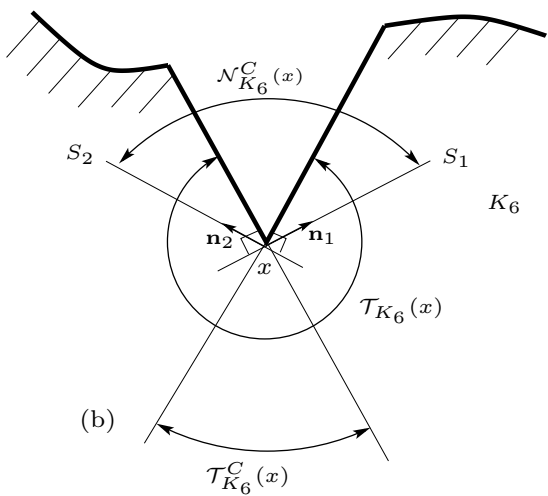

Fig. A.1: The closed sets $K_{3}, K_{4}, K_{5}$ and $K_{6}$.
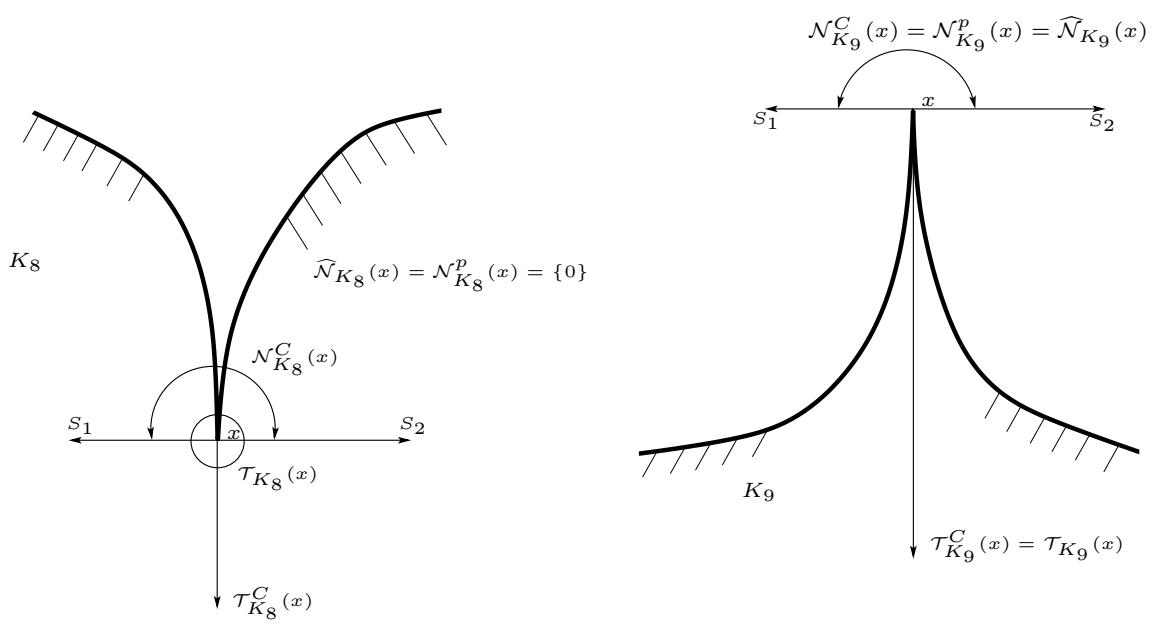

Fig. A.2: The closed sets $K_{8}$ and $K_{9}$.

$$
\mathcal{N}_{K}^{h}(x)=\left(\mathcal{T}_{K}^{h}(x)\right)^{\circ}=\left\{z \in \mathbb{R}^{n} \mid z=-\sum_{i \in \mathcal{I}(x)} \lambda_{i} \nabla k_{i}(x), \lambda_{i} \geq 0\right\}
$$

Both cones are convex closed polyhedral sets. Let $K$ be closed convex, then it is always true that $\mathcal{N}_{K}^{h}(x) \subseteq \mathcal{N}_{K}(x)$ and $\mathcal{T}_{K}(x) \subseteq \mathcal{T}_{K}^{h}(x)$. Suppose that there exists a vector $v \in \mathbb{R}^{n}$ such that $\nabla k_{i}(x)^{T} v>0$ for all $i \in \mathcal{I}(x)$ (this is the so-called MangasarianFromovitz constraint qualification (MFCQ); other CQs exist but the MFCQ is widely used). If the MFCQ holds, then $\mathcal{T}_{K}(x)=\mathcal{T}_{K}^{h}(x)$. If in addition convexity of $K$ holds, then $\mathcal{N}_{K}^{h}(x)=\mathcal{N}_{K}(x)$ (thus in the convex case both equalities are equivalent [133]). An important property of convex polyhedral sets defined as $\left\{x \in \mathbb{R}^{n} \mid C x+D \geq 0\right\}$, is that one can define the normal and tangent cones directly from their linearization cones [314, Examples 5.2.6, p.67] (but this is not true for all convex sets: Let $K=\{x \in$ $\left.\mathbb{R}^{n} \mid x^{T} x \leq 0\right\}$, then $K=\{0\}, \mathcal{T}_{K}^{h}(0)=\mathbb{R}^{n}$ but from (A.1) $\left.\mathcal{T}_{K}(0)=\left(\mathcal{N}_{K}(0)\right)^{\circ}=\{0\}\right)$. For such convex polyhedral sets one has $\mathcal{N}_{K}(x)=\left\{w \in \mathbb{R}^{n} \mid w=-C_{\mathcal{I}}^{T} \cdot \lambda, \lambda \geq 0\right\}$, 
where $\mathcal{I} \in\{1, \ldots, m\}$ is the index set of active constraints, i.e., $C_{i} \bullet x+D_{i}=0$ for all $i \in \mathcal{I}$. Then $\mathcal{T}_{K}(x)=\left\{z \in \mathbb{R}^{n} \mid C_{\mathcal{I} \bullet} z \geq 0\right\}=\left(\mathcal{N}_{K}(x)\right)^{\circ}$. In fact the Clarke tangent and normal cones can also be expressed in a linearization form under a regularity assumption and the MFCQ [191, p.131 and p.172]. Let $K=\left\{x \in \mathbb{R}^{n} \mid k(x) \geq 0, h(x)=\right.$ $0\}, h: \mathbb{R}^{n} \rightarrow \mathbb{R}^{p}$, with $k(\cdot)$ and $h(\cdot)$ continuously differentiable (extension exists with just Lipschitz continuity). Then $\mathcal{T}_{K}(x) \subseteq \mathcal{T}_{K}^{h}(x)$. Assume that $[\gamma \geq 0$ and $\gamma^{T} k(x)=0$ and $\lambda^{T} \nabla h(x)=0$ imply $\gamma=0$ and $\left.\lambda=0\right]$. Then if $k(x)=0$, one has $\mathcal{T}_{K}^{C}(x)=\mathcal{T}_{K}(x)=\left\{z \in \mathbb{R}^{n} \mid \nabla k_{i}(x)^{T} z \geq 0(1 \leq i \leq m), \nabla h_{i}(x)^{T} z=0(1 \leq i \leq p)\right\}$, and $\mathcal{N}_{K}^{C}(x)=$ cone $\left\{z=-\nabla k(x) \gamma+\nabla h(x) \lambda, \gamma \in \mathbb{R}^{m}, \lambda \in \mathbb{R}^{p}, \gamma \geq 0\right\}$. Under the MFCQ: the gradients $\nabla h_{i}(x), 1 \leq i \leq p$ are independent, and there exists a vector $v$ such that $\nabla h_{i}(q)^{T} v=0,1 \leq i \leq p, \nabla k_{i}(x)^{T} v>0$ for all $i \in \mathcal{I}(x)$, one has also $\mathcal{T}_{K}^{C}(x)=\mathcal{T}_{K}^{h}(x)$ and $\mathcal{N}_{K}^{C}(x)=\widehat{\mathcal{N}}_{K}(x)$ [41, Theorem 3.4]. This means that for proxregular sets (see Definition A.2) which are finitely represented and satisfy the MFCQ, $\mathcal{N}_{K}^{p}(x), \widehat{\mathcal{N}}_{K}(x), \mathcal{N}_{K}(x)$ and $\mathcal{N}_{K}^{C}(x)$ (which are identical), as well as $\mathcal{T}_{K}^{C}(x)$, can all be expressed in a linearized form, which is quite interesting to build complementarity problems.

REMARK 28. The normal cone can be defined with a metric defined by $M=$ $M^{T} \succ 0$, replacing $\langle z, y\rangle \leq 0$ by $\langle z, y\rangle_{M}=z^{T} M y \leq 0$ in the polarity definition. This gives $\mathcal{N}_{K}^{M}(x)=M^{-1} \mathcal{N}_{K}(x)$. In case the MFCQ holds, $z=\sum_{i=1}^{m} \lambda_{i} M^{-1} \nabla k_{i}(x)$ in (A.4).

\section{A.2. Other definitions and results.}

A.2.1. Maximal monotonicity, subdifferentials, prox-regular sets. Let us start with the classical maximal monotone operator definition.

Definition A.1 (Maximal monotone operator [97]). An operator $A: \mathbb{R}^{n} \rightrightarrows \mathbb{R}^{n}$ is monotone if for all $x_{1} \in \operatorname{dom}(A), x_{2} \in \operatorname{dom}(A), y_{1} \in A\left(x_{1}\right), y_{2} \in A\left(x_{2}\right)$, one has $\left\langle x_{1}-x_{2}, y_{1}-y_{2}\right\rangle \geq 0$. It is strongly monotone if there exists $c>0$ such that $\left\langle x_{1}-x_{2}, y_{1}-y_{2}\right\rangle \geq c\left\|x_{1}-x_{2}\right\|^{2}$. It is maximal if it cannot be extended without destroying the monotonicity. It is c-hypomonotone if there exists $c>0$ such that $\left\langle x_{1}-x_{2}, y_{1}-y_{2}\right\rangle \geq-c\left\|x_{1}-x_{2}\right\|^{2}$.

Let $\varphi: \mathbb{R}^{n} \rightarrow \mathbb{R} \cup\{+\infty\}$ be a proper LSC convex function. Then its subdifferential $\partial \varphi: \mathbb{R}^{n} \rightrightarrows \mathbb{R}^{n}$ is the set of its subgradients $\eta \in \mathbb{R}^{n}$, defined as: $\eta \in \partial \varphi(x)$ if $\langle\eta, y-x\rangle+\varphi(x)-\varphi(y) \leq 0$ for all $y \in \mathbb{R}^{n}$. It defines a maximal monotone operator $[97,492]$. The indicator function of $K$ is defined as $\psi_{K}(x)=0$ if $x \in K, \psi_{K}(x)=+\infty$ if $x \notin K$. It is convex proper and LSC when $K$ is closed convex non empty. One has

$$
\partial \psi_{K}(x)=\mathcal{N}_{K}(x)
$$

whenever $K$ is closed nonempty convex. There are extensions of subdifferentials for non convex functions. One way of defining them is as follows. Let $\varphi: \mathbb{R}^{n} \rightarrow$ $\mathbb{R} \cup\{+\infty\}$ be an extended real valued function. Then its proximal (resp. Fréchet, Mordukovich limiting, Clarke) subdifferential at $x$ with $f(x)<+\infty$ is defined by stating that subgradients $\eta \in \partial_{p} f(x)$ (resp. $\left.\partial_{F} f(x), \partial_{L} f(x), \partial_{C} f(x)\right)$ when $(\eta,-1)$ belongs to the corresponding normal cone (proximal, Fréchet, Mordukhovich, Clarke) to $\operatorname{epi}_{f}(x, f(x))=\left\{(x, y) \in \mathbb{R}^{n} \times \mathbb{R} \mid y \geq f(x)\right\}$ [191, Theorem 5.7] [431]. Thus the rationale behind proximal subdifferentiation is to approximate (locally) a function by a reversed parabola, instead of a straight line as in convex analysis. Similarly to normal cones, one has $\partial_{p} f(x) \subset \partial_{F} f(x) \subset \partial_{L} f(x) \subset \partial_{C} f(x)$. See Figures A.1 and A.3 and Example 11 for illustrating examples. Given a closed subset $K \subseteq \mathbb{R}^{n}$, the Fréchet (resp. basic) subdifferential of its indicator function $\psi_{K}(\cdot)$ at $x \in K$ is the Fréchet 
(resp. basic) normal cone $\widehat{\mathcal{N}}_{K}(x)$ (resp. $\mathcal{N}_{K}^{s}(x)$ ), that is $\partial_{F} \psi_{K}(x)=\widehat{\mathcal{N}}_{K}(x)$ (resp. $\left.\partial_{L} \psi_{K}(x)=\mathcal{N}_{K}^{s}(x)\right)$. Also $\partial_{p} \psi_{K}(x)=\mathcal{N}_{K}^{p}(x)$. These equalities generalize (A.5) to closed non convex sets. A function is normally regular at $x$ if its epigraph is normally regular at $(x, f(x))[143,431,492]$.

Definition A.2 (Prox-regular set [102]). A set $K \subset \mathbb{R}^{n}$ is called uniformly proxregular with constant $\frac{1}{r}$, or simply $r$-prox-regular, if one of the following equivalent properties is satisfied: (i) for each $x \in K$ and each $w \in \widehat{\mathcal{N}}_{K}(x)$ with $\|w\|<1$, it holds that $\operatorname{proj}(K ; x+r w)=\{x\}$, that is, $x$ is the unique nearest vector to $x+r w$ in $K$. (ii) For each $x, y$ in $K$ and each $w \in \widehat{\mathcal{N}}_{K}(x)$ with $\|w\|<1$, one has $\|r w\|^{2}<\| x-$ $y\left\|^{2}+2\langle r w, x-y\rangle+\right\| r w \|^{2}$. (iii) For all $x_{1} \in K, x_{2} \in K$, for each $z_{1} \in \widehat{\mathcal{N}}_{K}\left(x_{1}\right) \cap \mathbb{B}_{\mathbb{R}^{n}}$, $z_{2} \in \widehat{\mathcal{N}}_{K}\left(x_{2}\right) \cap \mathbb{B}_{\mathbb{R}^{n}}:\left\langle z_{1}-z_{2}, x_{1}-x_{2}\right\rangle \geq-\frac{1}{r}\left\|x_{1}-x_{2}\right\|^{2}$. (iv) For each $x \in K$ and each $w \in \widehat{\mathcal{N}}_{K}(x):\left\langle\frac{w}{\|w\|}, x-y\right\rangle \geq-\frac{1}{2 r}\|x-y\|^{2}$ for all $y \in K$.

Property (iii) means that the operator $\widehat{\mathcal{N}}_{K}(x) \cap \mathbb{B}_{\mathbb{R}^{n}}$ is $\frac{1}{r}$-hypomonotone. In view of the equality of normal cones for prox-regular sets (see Section A.1), all results about proxregular sets can be formulated with any of the normal cones $\mathcal{N}_{K}^{p}(x), \widehat{\mathcal{N}}_{K}(x), \mathcal{N}_{K}(x)$, $\mathcal{N}_{K}^{C}(x)$. When $r \rightarrow+\infty$ one recovers the convex case with maximal monotonicity of the normal cone operator, thus $r$ may be seen as a sort of measure of non-convexity (this is very useful in stability analysis [522]). However prox-regular sets are far from being convex since, for instance, intersection of prox-regular sets may fail to be prox-regular. An interesting property of $r$-prox regular sets is as follows: for any $x \in\left\{z \in \mathbb{R}^{n} \mid \inf _{s \in K}\|s-z\|<r\right\}$, the orthogonal projection proj $[K ; x]$ is uniquely defined. In other words, the projection onto an $r$-prox regular set is unique for all points "close enough" (in a "tube" of radius $r$ ) to the set. The sets $K_{3}, K_{4}$, and $K_{6}$ in Section A.1 are non convex, non prox-regular cones. Prox-regular sets may have smooth or non-differentiable boundary. It is of interest to characterize finitely represented sets which are $r$-prox regular, in terms of the functions $k_{i}(\cdot)$, see [41] for several results (the hypomonotonicity of the gradients $\nabla k_{i}(x)$ is a core property), see also [143, Section 4.3]. Finally we recall that one can also define prox-regular functions [102, Definition 2.1]. Then a set $K \subseteq \mathbb{R}^{n}$ is $r$-prox-regular if its indicator function $\psi_{K}(\cdot)$ is prox-regular [102, Definition 2.2], and then $\partial \psi_{K}(x)=\mathcal{N}_{K}(x)$ (the set of Fréchet subgradients of $\left.\psi_{K}(\cdot)\right)$, generalizing (A.5).

REMARK 29. The above material shows that provided some basic assumptions are made on the relevant sets and functions, it is possible to express tangent, normal cones and subdifferentials of the indicators functions in rather simple and practical ways, involving the gradients of the active constraints and complementarity conditions with non negative mutlipliers. However in a more general setting, calculations may not be straightforward. See [81,350] who developed automatic differentiation tools for evaluating generalized derivatives.

EXAMPLE 11. In order to illustrate the importance of differentiability of the "gap" functions $k_{i}(\cdot)$ defining finitely represented sets, let us consider the set $K=\{(x, y) \in$ $\left.\mathbb{R}^{2}|k(x, y)=y+| x \mid \geq 0\right\}[338,295]$. The function $k(x, y)$ is Lipschitz continuous, non differentiable at $x=0 . K$ is the union of two convex sets $K^{+}=\{(x, y) \mid y \geq-x, x \geq 0\}$ and $K^{-}=\{(x, y) \mid y \geq x, x \leq 0\}$, and has a reentrant corner as $K_{6}$ in Figure A.1. The $M F C Q$ is satisfied at $(0,0)$ and thus $\mathcal{N}_{K}^{C}(0,0)$ can be expressed in a linearized form. We note that $K$ is the epigraph of the non convex function $\varphi: \mathbb{R} \rightarrow \mathbb{R}$, such that $y=\varphi(x), x \mapsto-x$ if $x \geq 0$ and $x \mapsto x$ if $x \leq 0$, i.e., $\varphi(x)=-|x|$. This is depicted in Figure A.3 (a). We see that the Fréchet and proximal subdifferentials of $\varphi(\cdot)$ do not exist at $(0,0)$ (since both normal cones are reduced to $\{0\}$ ). The Mordukhovich 
normal cone is $\mathcal{N}_{\mathrm{epi}}^{s}(0,0)=S_{1} \cup S_{2}$, where $S_{i}, i=1,2$, is the half-line emerging from the origin as indicated in Figure A.3 (a). Thus $\partial_{L} \varphi(0,0)=\{-1,1\}$ (two elements). One has $\partial_{C} \varphi(0)=[-1,1]$ and the Clarke normal cone to the epigraph as depicted is such that for all $\eta \in[-1,1]$, the vector $(\eta,-1)$ belongs to it. If instead we consider a function $\varphi(\cdot)$ such that the corner is rounded with positive radius $r$ (dashed curve in Figure A.3 (a)), then its epigraph is $r$-prox-regular and so is $\varphi(\cdot)$. A slightly more complex case is $\varphi: \mathbb{R}^{2} \rightarrow \mathbb{R}$, with $f(x, y)=|x|-|y|$. Then $\partial_{F} \varphi(0,0)=\emptyset$ (Fréchet subdifferential does not exist), $\partial_{L} \varphi(0,0)=\{-1,1\}$, and $\partial_{C} \varphi(0,0)=[-1,1]$.

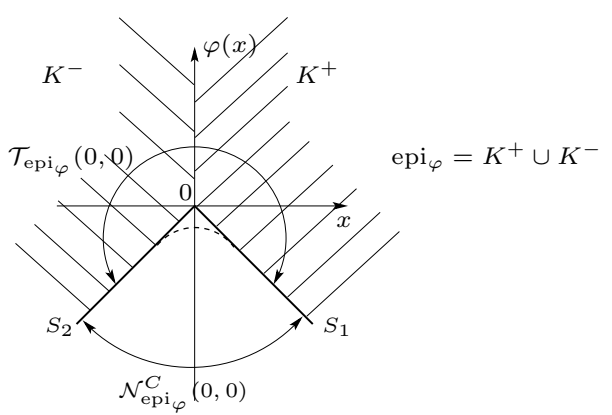

(a)

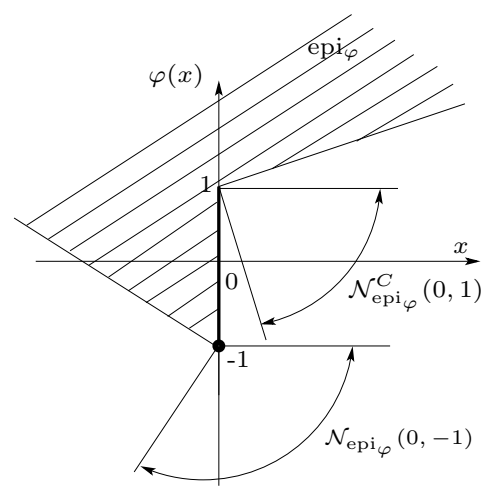

(b)

Fig. A.3: Examples 11 and 12.

EXAMPLE 12. Let us consider the function $\varphi: \mathbb{R} \rightarrow \mathbb{R}$ such that $\varphi(x)=-x-1$ if $x \leq 0, \varphi(x)=a x+1$ if $x>0, a \geq 0$. It is discontinuous at $x=0$ but is $L S C$, equivalently epi $i_{\varphi}$ is closed, see Figure A.3 (b). We have $\partial_{C} \varphi(0,1)=[a,+\infty)$, $\partial_{C} \varphi(0,-1)=[-1,+\infty)$. We see that epi $i_{\varphi}$ has a reentrant corner at $(0,1)$. If we compute the derivative of $\varphi(\cdot)$ in the sense of distributions [503], then we obtain $d \varphi=2 \delta_{0}+g(x) d x$ for some function $g(x) \stackrel{\text { a.e. }}{=} \dot{\varphi}(x)$. In this article we encounter both types of generalized derivatives: subdifferentials and distributions (or measures).

Other types of sets are used (for instance for FOSwP), like uniformly subsmooth and $\alpha$-far sets [339, 338], which are extensions of prox-regular sets, with the hypomonotonicity replaced by $\left\langle z_{1}-z_{2}, x_{1}-x_{2}\right\rangle \geq-\left\|x_{1}-x_{2}\right\|$. We may nevertheless conclude from Sections A.1.1, A.1.2 and A.2.1 that there are four main types of sets which deserve close attention due to their practical usefulness and their mathematical properties: convex, prox-regular, polyhedral and finitely represented closed sets.

A.2.2. Generalized equations, variational inequalities. According to [489] a generalized equation is a nonlinear problem $0 \in G(x, y)+F(x, y)$ where $F: \mathbb{R}^{n \times n} \rightrightarrows$ $\mathbb{R}^{m}$ is a set-valued mapping, $G: \mathbb{R}^{n \times n} \rightarrow \mathbb{R}^{n}$ is a continuously differentiable mapping. Let us now present some classical generalized equations. Let $M=M^{T} \succ 0, K \subseteq \mathbb{R}^{n}$ 
a closed non empty convex set, $x \in \mathbb{R}^{n}, y \in \mathbb{R}^{n}$, then:

$$
\begin{gathered}
M(x-y) \in-\mathcal{N}_{K}(x) \Leftrightarrow x=\operatorname{proj}_{M}[K ; y] \Leftrightarrow x=\arg \min _{z \in K} \frac{1}{2}(z-y)^{T} M(z-y) \\
\Leftrightarrow \text { Find } x \in \mathbb{R}^{n} \text { such that: }\langle M(x-y), y-x\rangle \geq 0 \text { for all } y \in K \\
\Leftrightarrow x=\left(M+\mathcal{N}_{K}\right)^{-1}(M y),
\end{gathered}
$$

where $\operatorname{proj}_{M}[K ; \cdot]$ denotes the orthogonal projection on $K$ in the metric defined by $M$ (in case $M$ is the identity we omit to write it), see Section A.2.4 for inverse mappings. The fourth formulation in (A.6), is a variational inequality (VI) of the first kind. The proof of (A.6) can be found in [240, 279]. An extension uses VI of the second kind. Let $P \succ 0, f(\cdot)$ be proper convex LSC, $r \in \mathbb{R}^{n}$. The $V I$ of second kind is: Find $x \in \mathbb{R}^{n}$ such that $\langle P x-r, \eta-x\rangle+f(\eta)-f(x) \geq 0$ for all $\eta \in \mathbb{R}^{n}$. This is equivalent to the inclusion $P x-r \in-\partial f(x)$, from which we easily recover from the problem in (A.6) if $f(\cdot)=\psi_{K}(\cdot)$ and $r=M y$. Its unique solution is given by $x=\operatorname{Prox}_{\mu f}[(I-\mu P) x+\mu r]$ for some $\mu>0$, where $\operatorname{Prox}_{\mu f}(\cdot)$ is the so-called proximal map of $f(\cdot)$ at $x[97,279]$. For instance if $f(x)=\|x\|_{1}$ then $\operatorname{Prox}_{\mu f}(x)=x-\operatorname{proj}\left[[-\mu, \mu]^{n} ; x\right]$. If $f(x)=\psi_{K}(x)$ with $K$ closed nonempty convex, $\operatorname{Prox}_{f}(x)=\operatorname{proj}[K ; x]$. See [208, Table 10.1] for more examples. Also $p=\operatorname{Prox}_{f}(x) \Leftrightarrow x-p \in \partial f(p) \Leftrightarrow p \in(I+\partial f)^{-1}(x)$ (which is similar to the last equality in (A.6)).

EXAMPLE 13. Consider the scalar DI: $\dot{x} \in-\operatorname{sgn}(x)$ and its implicit Euler discretization $x_{k+1}-x_{k} \in-h \operatorname{sgn}\left(x_{k+1}\right)=-\partial\left(h\left|x_{k+1}\right|\right)$. We have $x_{k+1}=\operatorname{Prox}_{h|\cdot|}\left(x_{k}\right)=$ $x_{k}-\operatorname{proj}\left[[-h, h] ; x_{k}\right]=-\operatorname{Prox}_{h \mu|\cdot|}\left[(\mu-1) x_{k+1}-\mu x_{k}\right]$ for $\mu>0$. Consider now the scalar first-order Moreau's sweeping process: $\dot{x} \in-\mathcal{N}_{K(t)}(x)=-\partial \psi_{K(t)}(x)$. Its implicit Euler discretization reads $x_{k+1}=x_{k}-\mathcal{N}_{K\left(t_{k+1}\right)}\left(x_{k+1}\right)$ (the time step plays no role because the right-hand side is a cone). Then $x_{k+1}=\operatorname{Prox}_{\psi_{K\left(t_{k+1}\right)}}\left(x_{k}\right)=$ $\operatorname{proj}\left[K\left(t_{k+1}\right) ; x_{k}\right]$. Such schemes are used in digital sliding-mode control [422, 421, 424] and contact mechanics [4].

Another type of VIs are the quasi VI (QVI) defined as follows. Let $K: \mathbb{R}^{n} \rightrightarrows \mathbb{R}^{n}$ be a set-valued mapping, and $f: C \rightarrow \mathbb{R}^{n}, C \subseteq \mathbb{R}^{n}$ a closed convex non empty set. The $\mathrm{QVI}(K, f)$ is: Find $x \in K(x)$ such that

$$
\langle f(x), y-x\rangle \geq 0 \text { for all } y \in K(x) .
$$

Again, different variants of QVIs exist [240]. The so-called hemi VI (HVI) are defined as [282]: find $x \in F(x), y \in G(x)$, such that $\langle y, v-x\rangle+J^{0}(x, v-x) \geq\langle f, v-x\rangle$ for all $v \in F(x)$, where $F(\cdot)$ and $G(\cdot)$ are set-valued mappings, $J(\cdot)$ is a locally Lipschitz mapping, $J^{0}(x, \cdot)$ is its Clarke's directional differential, $f$ is arbitrary (a vector in finite dimensions). Other HVI are of the form: find $x \in \mathbb{R}^{n}$ such that $\langle A(x)-f, v-x\rangle+J^{0}(x, v-x) \geq 0$ for all $v \in \mathbb{R}^{n}, A(\cdot)$ a monotone operator. This HVI is equivalently formulated as the GE: $A(x)+\partial J(x) \ni f$, where $\partial J(\cdot)$ is the Clarke's subdifferential [282]. Thus, HVIs extend VIs of the second kind, which are closely linked with the subdifferential of convex analysis, to Clarke's differentiation, see [163] for a complete exposition.

A.2.3. Chain rules. Chain rules for the differentiation of composed nonsmooth functions are commonly used in some parts of the article. We recall that a polyhedral function, is a function whose epigraph is a closed convex polyhedral set. The indicator function $\psi_{K}(\cdot)$ is polyhedral if $K$ is closed convex polyhedral. 
Proposition A.3. [97, 314, 492] Let $f: \mathbb{R}^{m} \rightarrow \mathbb{R} \cup\{+\infty\}$ be a proper convex LSC function, and $A: \mathbb{R}^{n} \rightarrow \mathbb{R}^{m}$ be a linear operator. Assume that either the function $f(\cdot)$ is polyhedral or for some $x_{0}$ with $A x_{0} \in \operatorname{dom}(f)$

$$
\operatorname{Im}(A)-\mathbb{R}_{+}\left(\operatorname{dom}(f)-A x_{0}\right) \quad \text { is a vector subspace of } \mathbb{R}^{m},
$$

where $\operatorname{dom}(f) \triangleq\left\{y \in \mathbb{R}^{m} \mid f(y)<+\infty\right\}$. Then the subdifferential in the sense of convex analysis of the composite functional $f \circ A: \mathbb{R}^{n} \rightarrow \mathbb{R} \cup\{+\infty\}$ is given by

$$
\partial(f \circ A)(x)=A^{T} \partial f(A x), \quad \forall x \in \mathbb{R}^{n} .
$$

Example 14. [62, Proposition 10] Let $\tilde{K}=\left\{x \in \mathbb{R}^{n} \mid H^{-1} x \in K\right\}=H K$, $H \in \mathbb{R}^{n \times m}$ full rank, $K \subseteq \mathbb{R}^{n}$ closed convex. Then $\mathcal{N}_{\tilde{K}}(x)=H^{-T} \mathcal{N}_{K}(x)$. Indeed $\psi_{K}(x)=\psi_{\tilde{K}}(H x)=\left(\psi_{\tilde{K}} \circ H\right)(x)$. By Proposition A.3, $\partial \psi_{\tilde{K}}(H x)=H^{T} \partial \psi_{\tilde{K}}(H x)=$ $H^{T} \mathcal{N}_{\tilde{K}}(H x)=\partial \psi_{K}(x)=\mathcal{N}_{K}(x)$, where $(A .5)$ has been used.

Part (a) of the next proposition is extracted from [430, Theorem 3.41] or [492, Theorem 10.6] and (b) is a consequence of (a). Before this, we need two definitions. A function $f(\cdot)$ is said subdifferentially regular if its epigraph epi ${ }_{f}$ is normally regular, that is $\mathcal{N}_{\text {epi }_{f}}^{s}(x, f(x))=\widehat{\mathcal{N}}_{\mathrm{epi}_{f}}(x, f(x))$ for all $x \in \operatorname{dom}(f)$ (see Section A.1). Thus convex functions are subdifferentially regular, and so are all functions such that their epigraph is prox-regular. The singular subdifferential of $f(\cdot)$ at $x$ is defined as $\partial^{\infty} f(x)=\{v \in$ $\left.\mathbb{R}^{n} \mid(v, 0) \in \mathcal{N}_{\mathrm{epi}_{f}}^{s}(x, f(x))\right\}$.

EXAMPLE 15. Let $f(x)=|x|$, then $\partial^{\infty} f(x)=\{(0,0)\}$ for all $x$. Let $f(\cdot)=\varphi(\cdot)$ in Figure A.3 (a), then epi is not normally regular so $f(\cdot)$ is not subdifferentially regular, and $\partial^{\infty} f(x)=\{(v, 0) \in \mathbb{R} \times \mathbb{R} \mid v=0\}$.

Proposition A.4. Suppose $g=f \circ F$ for a proper LSC function $f: \mathbb{R}^{m} \rightarrow$ $\mathbb{R} \cup\{+\infty\}$, and a mapping $F: \mathbb{R}^{n} \rightarrow \mathbb{R}^{m}$ which is continuously differentiable at a point $x$ where $g(\cdot)$ is finite.

(a) If $f(\cdot)$ is subdifferentially regular at $F(x)$ and if

$$
\partial^{\infty} f(F(x)) \cap \operatorname{Ker} \nabla F(x)=\{0\},
$$

then $g(\cdot)$ is subdifferentially regular at $x$, and

$$
\partial g(x)=\nabla F(x) \partial f(F(x)) .
$$

(b) In particular, the conclusions of (a) hold whenever $f(\cdot)$ is convex and

$$
\operatorname{Im}\left(\nabla F(x)^{T}\right)+\mathbb{R}_{-}(\operatorname{dom} f-F(x))=\mathbb{R}^{m} .
$$

In (A.11) the subdifferential is that of Mordukovich (see Section A.2.1). See [301, Proposition 1] for another variation of the chain rule. From [492, Theorem 10.6] condition (A.10) holds true for convex $f(\cdot)$ if $\operatorname{dom}(f)$ cannot be separated from the range of the affine function $w \mapsto F(x)+\nabla F(x)^{T} w$.

EXAMPLE 16. Let us show one application of Proposition A.4, which is useful to show relationships between complementarity systems and the FOSwP, amongst other applications. Let $K \subseteq \mathbb{R}^{m}$ be a prox-regular set, $\mathcal{S}(t)=\left\{z \in \mathbb{R}^{n} \mid k(z)+h(u(t)) \in\right.$ 
$K\} \subseteq \mathbb{R}^{n}$ and define $\phi_{t}: \mathbb{R}^{n} \rightarrow \mathbb{R}^{m}: z \mapsto k(z)+h(u(t))$, where $u: \mathbb{R} \rightarrow \mathbb{R}^{p}$. Let $k(\cdot)$ and $K$ be such that $\mathcal{S}(t)$ is prox-regular for each $t$. Let $g(\cdot)=\psi_{K} \circ \phi_{t}(\cdot)$, and assume that $\operatorname{ker}(\nabla k(z))=\{0\}$ for all $z \in \mathbb{R}^{n}$. Thus the conditions of Proposition A.4 (a) are satisfied. Then $\partial g(z)=\nabla \phi_{t}(z) \partial \psi_{K}\left(\phi_{t}(z)\right)$. In addition $g(z)=\psi_{\mathcal{S}(t)}(z)$, so that $\partial g(z)=\mathcal{N}_{\mathcal{S}(t)}(z)$, where the normal cone may be Clarke, proximal, or Fréchet normal cone (all equal in this case since the set is prox-regular).

A.2.4. Conjugate functions, inverse set-valued mappings. Let $f: \mathbb{R}^{n} \rightarrow$ $\mathbb{R} \cup\{+\infty\}$ be convex, proper and LSC. Its conjugate (or Legendre, or Legendre-Fenchel transform, or Fenchel conjugate) function $g: \mathbb{R}^{n} \rightarrow \mathbb{R} \cup\{+\infty\}$ is defined as

$$
g(y)=\sup _{x \in \mathbb{R}^{n}}[\langle x, y\rangle-f(x)],
$$

and is usually denoted as $g(y)=f^{*}(y)$. It is proper, convex LSC. One has $g^{*}(\cdot)=$ $f(\cdot)$ (as a corollary of the Fenchel-Moreau's Theorem [97, Theorem 13.32, Corollary 13.33]). One typical example encountered throughout the article, is the indicator $f(x)=\psi_{K}(x), K \subseteq \mathbb{R}^{n}$ a closed convex non empty set, and the support function of $K: \sigma_{K}(y)=\psi_{K}^{*}(y)$. See [97, chapter 13] for a complete presentation of conjugacy. This is quite related to inverse (set-valued) mappings: if $f(\cdot)$ is proper convex LSC, then $(\partial f)^{-1}=\partial f^{*}\left[97\right.$, Corollary 16.24]. In other words, $y \in \partial f(x) \Leftrightarrow x \in \partial f^{*}(y)$. Therefore, $\left(\partial \psi_{K}\right)^{-1}=\left(\mathcal{N}_{K}\right)^{-1}=\partial \psi_{K}^{*}=\partial \sigma_{K}$, where we used (A.5).

B. Some tools from complementarity theory. The reference book for this topic is [210], see also [240, 278].

Definition B.1 (Complementarity Problem). Let $F: \mathbb{R}^{n} \rightarrow \mathbb{R}^{n}$ and $\lambda \in \mathbb{R}^{n}$. The problem $\lambda \geq 0, F(\lambda) \geq 0, \lambda^{T} F(\lambda)=0$ is a complementarity problem (CP) with unknown $\lambda$, written compactly as $0 \leq \lambda \perp F(\lambda) \geq 0$. When $F(\lambda)=M \lambda+q$ for a matrix $M$ and a vector $q$, this is a linear $C P(L C P)$, denoted $L C P(q, M)$. The set of solutions (possibly empty) of $L C P(q, M)$ is denoted $\operatorname{SOL}(\operatorname{LCP}(q, M))$.

Definition B.2. A matrix $M \in \mathbb{R}^{n \times n}$ is positive (semi) definite if for all $x \in \mathbb{R}^{n}$ one has $x^{T} M x>0(\geq 0)$ for all $x \neq 0$. It is denoted $M \succ 0(\succcurlyeq 0)$. It is not necessarily symmetric. A matrix $M \in \mathbb{R}^{n \times n}$ is a P-matrix if all its principal subdeterminants (or principal minors) are positive. It is a $P_{0}$-matrix if its principal minors are non negative. It is a copositive matrix on the set $K$ if $x^{T} M x \geq 0$ for all $x \in K$.

We have $M \succ 0 \Rightarrow M$ is a $\mathrm{P}$-matrix, $M \succcurlyeq 0 \Rightarrow M$ is a $\mathrm{P}_{0}$-matrix and a copositive matrix on $\mathbb{R}_{+}^{n}$. One usually considers copositivity over convex sets [315], even in this case copositivity is hard to characterize. Many more matrix classes which are useful in complementarity theory exist [210]. The following result is central in complementarity theory.

TheOREM B.3. The $\operatorname{LCP}(q, M)$ has a unique solution for any $q$, if and only if $M$ is a P-matrix.

Copositive matrices can guarantee the solvability under some conditions on the homogenous $\operatorname{LCP}(0, M)$ :

Theorem B.4. (i) Suppose $M$ is copositive on $\mathbb{R}_{+}^{n}$. If the implication $[0 \leq \nu \perp$ $\left.M \nu \geq 0 \Rightarrow q^{T} \nu \geq 0\right]$ holds, the $\operatorname{LCP}(q, M)$ has a solution. (ii) The $\operatorname{LCP}(q, M)$ has a unique solution for all non-negative vectors $q$, if and only if $M$ is strictly semicopositive (i.e., $\max _{1 \leq i \leq n} z_{i}(M z)_{i} \geq \sigma\|z\|^{2}$ for all $z$ and some $\sigma>0$ ).

Positive semi definite matrices also possess interesting properties:

Theorem B.5. Suppose that $M=M^{T} \succcurlyeq 0$. If $\lambda_{1}$ and $\lambda_{2}$ are two solutions of $L C P(q, M)$, then $M\left(\lambda_{1}-\lambda_{2}\right)=0$. 
There are several other types of complementariry problems like horizontal, mixed (MLCP), vertical, geometric, generalized, cone (LCCP), nonlinear (NLCP) complementarity problems, see for instance [4, Chapter 12]. An interesting fact is: let $K \subseteq \mathbb{R}^{n}$ be a closed convex cone, then $[278$, p.18]

$$
K^{\star} \ni x \perp y \in K \Leftrightarrow x \in-\mathcal{N}_{K}(y) \Leftrightarrow y \in-\mathcal{N}_{K^{\star}}(x),
$$

for any two vectors $x$ and $y$. This can be used in (A.6) to derive another equivalent formulation. If $y=M x+q$, the left-hand side of (B.1) is a linear cone complementarity problem (LCCP), whose set of solutions is denoted $\operatorname{SOL}(K, q, M)$. Let $M=M^{T} \succ 0$, using (A.6) the LCCP is equivalent to an optimization problem with $x=\arg \min _{z \in K^{\star}} \frac{1}{2}\left(z^{T} M z+q^{T} z\right)$. Solvability and uniqueness of solutions for LCCP with $M$ copositive on $K$, are analysed in [11]. For instance, the next result follows from [11, Corollary 5] [279, Corollary 9 p.105]. Let $\mathcal{B}\left(M, K^{\star}\right) \triangleq\left\{z \in \mathbb{R}^{n} \mid M z \in K, z^{T} M z=\right.$ $0\}$, and assume that $x^{T} M x \geq 0$ for all $x \in K^{\star}$. Then (i) if $\mathcal{B}\left(M, K^{\star}\right)=\{0\}$, or (ii) if $\mathcal{B}\left(M, K^{\star}\right) \neq\{0\}$, and there exists $x_{0}$ such that $v^{T}\left(q-M^{T} x_{0}\right)>0$ for all $v \in \mathcal{B}\left(M, K^{\star}\right), v \neq 0$, the LCCP has at least one solution. Other results may be found in $[11,279]$ for $\mathrm{P}_{0}$ and P-matrices. This allows one to extend partially (A.6) to classes of matrices larger than symmetric positive definite matrices (in case of non uniqueness, one may define the projection operator as $\left.x \in\left(M+\mathcal{N}_{K}\right)^{-1}(M y)\right)$. The results in [11, 279] allow to analyse also the well-posedness of some MLCPs [137]. This could be useful for the study of equilibria sets (Section 6.1) and well-posedness of MLCS (Section 2.4.3).

Let us now provide the expression of the solutions of the LCP: $0 \leq \lambda \perp z=C x+D \lambda+$ $E_{3} u+E_{4} \geq 0$ in (2.22) when $D$ is a P-matrix. To this aim we introduce the index sets $\alpha(x, u)=\left\{i \mid \lambda_{i}>0, z_{i}=0\right\}, \beta(x, u)=\left\{i \mid \lambda_{i}=z_{i}=0\right\}, \gamma(x, u)=\left\{i \mid \lambda_{i}=0, z_{i}>0\right\}$ of the LCP modes, and $\bar{\alpha}(x, u)=\gamma(x, u) \cup \beta(x, u)$. Then $\lambda_{\alpha}(x, u)=-\left(D_{\alpha \alpha}\right)^{-1}\left(C_{\alpha} \bullet x+\right.$ $\left.E_{3 \alpha} \bullet+E_{4 \alpha}\right)$, and $\lambda_{\bar{\alpha}}(x, u)=0[157]$.

C. Relative degree. Roughly speaking, the relative degree of a system with inputs and outputs, is the number of times one has to differentiate the output, in order to recover the input. This is a notion very close to the index of semi-explicit DAEs. Consider the quadruple $(A, B, C, D)$ with constant matrices $A \in \mathbb{R}^{n \times n}, B \in \mathbb{R}^{n \times m}$, $C \in \mathbb{R}^{m \times n}, D \in \mathbb{R}^{m \times m}$. The ( $m$-vector) relative degree $\bar{r} \in \mathbb{N}^{m}$ between the output $y=C x+D u$ and the input $u$, is equal to $(0,0, \ldots, 0)^{T}$ if and only if $D$ has full rank. When $D=0, C A^{i-1} B=0$ for all $1 \leq i<r$, and the leading Markov parameter or decoupling matrix $C A^{r-1} B$ is full rank, then $\bar{r}=(r, r, \ldots, r)^{T}$ with $r \leq n$. This extends to nonlinear systems $\dot{x}=f(x)+g(x) u, y=h(x)$ [553]. The relative degree is fundamental in Automatic Control to derive the so-called normal form and the zero dynamics. The existence of a relative degree implies the existence of a diffeomorphic change of state space which transforms the controlled dynamics with output into the canonical form: $\dot{z}_{1}=z_{2}, \dot{z}_{2}=z_{3}, \ldots, \dot{z}_{r-1}=z_{r}, \dot{z}_{r}=a(z)+b(z) u$, $\dot{\zeta}=c\left(\zeta, z_{1}\right)$. The $\zeta$-dynamics is the zero dynamics, which represents the system's dynamics on the submanifold $z_{1}=0$. In the linear invariant case $b(z)=C A^{r-1} B$ and $a(z)=C A^{r} x=C A^{r} W^{-1} z$, and the zero-dynamics is linear as in (2.8c).

The notion of index of a transfer matrix $H(s) \in \mathbb{C}^{m \times m}$ is close to the relative degree and is defined as follows [306, Definition 3.5].

Definition C.1. A rational matrix $\mathcal{H}(s) \in \mathbb{R}^{l \times l}(s)$ is said to be of index $r$, if it is invertible as a rational matrix and $s^{-r} \mathcal{H}^{-1}(s)$ is proper. It is said to be totally of index $r$, if all its principal submatrices $\mathcal{H}_{J J}(s)$ for $J \subseteq\{1, \ldots, l\}$ are of index $r$. 
Consequently, in case $l=1$ the index is the usual relative degree of a rational transfer function. Principal submatrices are obtained by deleting rows and columns of equal index [104, p.41]. It is noteworthy that Definition C.1 does not necessarily define a unique $r$.

D. Dissipative systems. A complete exposition of dissipative systems can be found in [139].

Definition D.1. Let $A, B, C, D$ be constant matrices with appropriate dimensions. The quadruple $(A, B, C, D)$ is said to be passive if there exist matrices $L \in \mathbb{R}^{n \times m}, W \in \mathbb{R}^{m \times m}$, and a $n \times n$ matrix $P=P^{\top} \succcurlyeq 0$, such that:

$$
\left\{\begin{array}{l}
A^{\top} P+P A=-L L^{\top} \\
B^{\top} P-C=-W^{\top} L^{\top} \\
D+D^{\top}=W^{\top} W .
\end{array}\right.
$$

In this case, let $V(x)=\frac{1}{2} x^{\top} P x$ denote the corresponding energy storage function. The dissipation equality

$V\left(x\left(t_{1}\right)\right)-V(x(0))=\int_{0}^{t_{1}} \lambda(t)^{\top} z(t) d t-\frac{1}{2} \int_{0}^{t_{1}}\left(x^{\top}(t), \lambda^{\top}(t)\right) \mathcal{Q}\left(\begin{array}{c}x(t) \\ \lambda(t)\end{array}\right) d t, \quad \forall t_{1} \geq 0$

in terms of the matrix

$$
\mathcal{Q} \triangleq\left(\begin{array}{cc}
L L^{\top} & L W \\
W^{\top} L^{\top} & W^{\top} W
\end{array}\right) \succcurlyeq 0,
$$

then implies that

$$
V\left(x\left(t_{1}\right)\right)-V(x(0)) \leq \int_{0}^{t_{1}} \lambda(t)^{\top} z(t) d t
$$

For an LCS (2.22) one has $\lambda(t)^{\top} z(t)=0$ for all times outside state jumps. The system is said to be strictly passive when $\mathcal{Q}$ is positive definite, and lossless when $\mathcal{Q}=0$. The system is said to be state lossless when $L=0$ and input lossless when $W=0$. The system is dissipative, state dissipative, and input dissipative when $\mathcal{Q} \neq 0$, $L \neq 0$, or $W \neq 0$, respectively. Passivity is a particular case of dissipativity, and is closely related to positive real transfer functions through the Kalman-YakubovichPopov Lemma [139, Chapter 3]. We have the following [306, Theorem 3.14]:

Theorem D.2. Consider that $(A, B, C, D)$ is passive with a positive definite storage function and $\left(\begin{array}{c}B \\ D+D^{\top}\end{array}\right)$ has full column rank. Then $H(s)=C(s I-A)^{-1} B+D$ is totally of index 1 (in the sense of Definition C.1).

We see using (D.1) that a passive system with $D+D^{\top}=0 \Rightarrow W=0$ satisfies $P B=C^{\top} \Rightarrow C B=B^{\top} P B \succcurlyeq 0$. If $P \succ 0$ and $B$ has rank $m$, then $C B \succ 0$.

REMARK 30. The notion of monotonicity of a set-valued operator is ubiquitous in this article. In Systems and Control, an input/output operator $T: \mathcal{L}^{2}\left(\mathbb{R}^{m}\right) \rightarrow \mathcal{L}^{2}\left(\mathbb{R}^{m}\right)$ is said incrementally passive if for any $u_{1}$ and $u_{2}$ one has $\left\langle u_{1}-u_{2}, T\left(u_{1}\right)-T\left(u_{2}\right)\right\rangle \geq 0$. Thus both notions are the same: one applies to static operators (like feedback setvalued nonlinearities in Lur'e systems), the other one is for dynamical systems with inputs and outputs. 
E. Basic definitions, notation, and results. For a vector $x \in \mathbb{R}^{n}$, the lexicographical inequality $x \succ 0$ means that the first non zero entry of $x$ is $>0$, $x \succcurlyeq 0$ means that the first non zero entry of $x$ is $\geq 0$. Let $M \in \mathbb{R}^{n \times n}$, and $\alpha=\left\{\alpha_{1}, \alpha_{2}, \ldots, \alpha_{p}\right\} \subseteq\{1,2, \ldots, n\}$. Then $M_{\alpha \alpha} \in \mathbb{R}^{p \times p}$ is the principal submatrix of $M$ obtained by deleting rows and columns indexed in $\bar{\alpha}=\{1,2, \ldots, n\} \backslash \alpha$, $x_{\alpha}=\left(x_{\alpha_{1}}, \ldots, x_{\alpha_{p}}\right)^{T}, M_{\alpha} \in \mathbb{R}^{p \times n}$ is the submatrix obtained by deleting all rows in $\bar{\alpha}$. The principal subdeterminants or principal minors of $M$ are the determinants $\operatorname{det}\left(M_{\alpha \alpha}\right)$ of its principal submatrices.

Let an interval $I \subseteq \mathbb{R}$, and a function $f: I \rightarrow \mathbb{R}^{n}$ be given. The variation of $f(\cdot)$ over the interval $I$ is the supremum of $\sum_{i=1}^{k}\left|f\left(s_{i}\right)-f\left(s_{i-1}\right)\right|$ over the set of all finite sets of points $s_{0}<s_{1}<\cdots<s_{k}$ (called partitions) of $I$. When this supremum is finite, the mapping $f(\cdot)$ is said to be of bounded variation (BV) on $I$. We say that $f(\cdot)$ is of locally bounded variation (LBV), if it is of bounded variation on each compact subinterval of $I$. For a BV function $f(\cdot)$, it holds that the right and left limits of $f(\cdot)$ are defined everywhere, and we use the notation $f\left(t^{+}\right) \triangleq \lim _{s \searrow t} f(s)$ and $f\left(t^{-}\right) \triangleq$ $\lim _{s \nearrow t} f(s)$. RCLBV denotes right-continuous LBV functions. One associates with a BV function $f(\cdot)$ its differential measure $d f$ [427], which satisfies for any $a \leq b$ : $d f([a, b])=f\left(b^{+}\right)-f\left(a^{-}\right), d f([a, b))=f\left(b^{-}\right)-f\left(a^{-}\right), d f((a, b])=f\left(b^{+}\right)-f\left(a^{+}\right)$, $d f((a, b))=f\left(b^{-}\right)-f\left(a^{+}\right)$, and $d f\left(\{a\}=f\left(a^{+}\right)-f\left(a^{-}\right)\right.$(the jump of $f(\cdot)$ at $\left.a\right)$.

We denote by $\mathcal{L}^{1}\left(I, \mathbb{R}^{n} ; d \nu\right)$ the space of integrable functions from the interval $I$ to $\mathbb{R}^{n}$ with respect to the measure $d \nu$. If the measure is not specified then the integration is with respect to the Lebesgue measure. An absolutely continuous (AC) function $f: I \rightarrow \mathbb{R}^{n}$ is a function that can be written as $f(t)-f\left(t_{0}\right)=\int_{t_{0}}^{t} \dot{f}(s) d s$ for any $t_{0}, t \in I, t_{0} \leq t$, and some $\dot{f} \in \mathcal{L}^{1}\left(I, \mathbb{R}^{n}\right)$, which is considered as its derivative. The definition of an AC set is given in (A1), Section 5.1. A possibly discontinuous function is lower semicontinuous (LSC) if its epigraph is closed. A set-valued map $F: \mathbb{R}^{n} \rightrightarrows \mathbb{R}^{m}$ is upper semicontinuous (another denomination is outer semicontinuous when local boundedness holds) at $x_{0} \in \mathbb{R}^{n}$, if for any open neighborhood $N$ containing $F\left(x_{0}\right)$, there exists an open neighborhood $M$ of $x_{0}$ such that $F(M) \subset N$. The subdifferential of a convex proper function $\varphi: \operatorname{dom}(\varphi)=\mathbb{R}^{n} \rightarrow \mathbb{R}$ is locally bounded and takes values in a compact set, and it defines an outer semicontinuous mapping [314, Theorem 6.2.4]. A measure $d \mu$ is absolutely continuous (AC) with respect to another measure $d \nu$ if $d \nu(I)=0$ implies $d \mu(I)=0$. The measures $d \nu$ and $d \mu$ are absolutely equivalent if they are AC with respect to each other. By the Radon-Nikodym theorem, the density of Lebesgue measure $d t$ relative to $d \nu$, defined as $\frac{d t}{d \nu}(s)=\lim _{\varepsilon \rightarrow 0} \frac{d t((s-\varepsilon, s+\varepsilon))}{d \nu((s-\varepsilon, s+\varepsilon))}$, is then well-defined.

For a set-valued mapping $\mathcal{S}:\left[t_{0}, \infty\right) \rightrightarrows \mathbb{R}^{l}$, for some fixed $t_{0} \in \mathbb{R}$, the variation of $\mathcal{S}(\cdot)$ over an interval $\left[t_{0}, t\right]$ denoted by $v_{\mathcal{S}}(t)$, is defined as,

$$
v_{\mathcal{S}}(t) \triangleq \sup _{t_{0}=s_{0}<s_{1}<\cdots<s_{k}=t} \sum_{i=1}^{k} d_{\text {Haus }}\left(\mathcal{S}\left(s_{i}\right), \mathcal{S}\left(s_{i-1}\right)\right)
$$

where the supremum is taken over the set of all partitions of $\left[t_{0}, t\right]$.

E.1. Mazur's Lemma. Let $(X,\|\cdot\|)$ be a Banach space and let $\left\{x_{n}\right\}_{n \in \mathbb{N}}$ be a sequence in $X$ that converges weakly to some $x_{0} \in X$. There exists a function $N: \mathbb{N} \rightarrow \mathbb{N}$ and a sequence of sets of positive numbers $\left\{\alpha_{n}^{n}, \cdots, \alpha_{n}^{N(n)}\right\}_{n \in \mathbb{N}}$, with $\sum_{k=n}^{N(n)} \alpha_{n}^{k}=1$, such that the sequence $\left\{y_{n}\right\}_{n \in \mathbb{N}}$ defined by the convex combination $y_{n}=\sum_{k=n}^{N(n)} \alpha_{n}^{k} x_{k}$, converges strongly to $x \in X$, i.e., $\left\|y_{n}-x_{0}\right\| \rightarrow 0$ as $n \rightarrow \infty$. 
E.2. Discrete-time Gronwall-Bellman lemma. If $\left\{y_{k}\right\}_{k \in \mathbb{N}},\left\{f_{k}\right\}_{k \in \mathbb{N}}$, and $\left\{g_{k}\right\}_{k \in \mathbb{N}}$ are nonnegative sequences and $y_{k} \leq f_{k}+\sum_{1 \leq j<k} g_{j} y_{j}, \quad k \in \mathbb{N}$. Then

$$
y_{k} \leq f_{k}+\sum_{1 \leq j<k} f_{j} g_{j} \exp \left(\sum_{j \leq i<k} g_{i}\right), \quad k \in \mathbb{N} .
$$

If $f_{k}=f_{0}$ is constant for each $k \in \mathbb{N}$, it can be shown that $y_{k} \leq f_{0} \exp \left(\sum_{1 \leq i<k} g_{i}\right)$, $k \in \mathbb{N}$.

\section{REFERENCES}

[1] NI AWR Design Environment, Microwave Office Element Catalog, tech. report, National Instruments, El Segundo, CA, USA, 2019.

[2] V. Acary, O. Bonnefon, and B. Brogliato, Nonsmooth Modeling and Simulation for Switched Circuits, vol. 69 of Lecture Notes in Electrical Engineering, Springer, Dordrecht Heidelberg, 2011. Erratum at https://hal.inria.fr/hal-01311078.

[3] V. ACARY, M. Brémond, AND O. Huber, On solving contact problems with Coulomb friction: formulations and numerical comparisons, in Advanced Topics in Nonsmooth Dynamics, R.-I. Leine, V. Acary, and O. Bruls, eds., Transactions of the European Network for Nonsmooth Dynamics, Springer Int. Publishing AG, 2018, pp. 375-457.

[4] V. Acary and B. Brogliato, Numerical Methods for Nonsmooth Dynamical Systems, vol. 35 of Lecture Notes in Applied and Computational Mechanics, Springer Verlag, Berlin Heidelberg, 2008

[5] V. Acary and B. Brogliato, Implicit Euler numerical scheme and chattering-free implementation of sliding mode systems, Systems and Control Letters, 59 (2010), pp. 284-293.

[6] V. Acary, B. Brogliato, and D. Goeleven, Higher order Moreau's sweeping process: Mathematical formulation and numerical simulation, Mathematical Programming A, 113 (2008), pp. 133-217.

[7] V. Acary, H. De Jong, and B. Brogliato, Numerical simulation of piecewise-linear models of gene regulatory networks using complementarity systems, Physica D: Nonlinear Phenomena, 269 (2014), pp. 103-119.

[8] L. AdAm AND J. Outrata, On optimal control of a sweeping process coupled with an ordinary differential equation, Discrete and Continuous Dynamical Systems - Series B, 19 (2014), pp. 2709-2738.

[9] K. Addi, S. Adly, B. Brogliato, and D. Goeleven, A method using the appproach of Moreau and Panagiotopoulos for the mathematical formulation of non-regular circuits in electronics, Nonlinear Analysis: Hybrid Systems, 1 (2007), pp. 30-43.

[10] K. Add, S. AdLY, AND H. SAOUd, Finite-time Lyapunov stability analysis of evolution variational inequalities, Discrete and Continuous Dynamical Systems - Series A, 31 (2011), pp. 1023-1038.

[11] K. Addi, B. Brogliato, And D. Goeleven, A qualitative mathematical analysis of a class of linear variational inequalities via semi-complementarity problems. Applications in electronics, Mathematical Programming A, 126 (2011), pp. 31-67.

[12] K. Addi AND D. Goeleven, Complementarity and variational inequalities in electronics, in Operations Research, Engineering, and Cyber Security, N. Daras and T. Rassias, eds., vol. 113 of Springer Optimization and Its Applications, Springer International Publishing, 2017, pp. 1-43.

[13] S. ADLY, Attractivity theory for second order nonsmooth dynamical systems with application to dry friction, Journal of Mathematical Analysis and Applications, 322 (2006), pp. 10551070.

[14] S. AdLy, A Variational Approach to Nonsmooth Dynamics. Applications in Unilateral Mechanics and Electronics, Springer Briefs in Mathematics, Springer International Publishing, Cham, 2017.

[15] S. AdLY, A coderivative approach to the robust stability of composite parametric variational systems: applications in nonsmooth mechanics, Journal of Optimization Theory and Applications, 180 (2019), pp. 62-90.

[16] S. Adly, H. Attouch, and A. Савот, Nonsmooth Mechanics and Analysis, Adv. in Math. and Mech., Kluwer, 2006, ch. Finite time stabilization of nonlinear oscillators subject to dry friction, pp. 289-304. 
[17] S. Adly, B. Brogliato, And B.-K. Le, Well-posedness, robustness and stability analysis of a set-valued controller for Lagrangian systems, SIAM Journal on Control and Optimization, 51 (2013), pp. 1592-1614.

[18] S. AdLY AND R. CibulKa, Quantitative stability of a generalized equation. application to nonregular electrical circuits, Journal of Optimization Theory and Applications, 160 (2014), pp. $90-110$.

[19] S. Adly, R. Cibulka, And H. Massias, Variational analysis and generalized equations in electronics. Stability and simulation issues, Set Valued and Variational Analysis, 21 (2013), pp. 333-358.

[20] S. AdLy AND D. Goeleven, A stability theory for second-order nonsmooth dynamical systems with application to friction problems, Journal de Mathématiques Pures et Appliquées, 83 (2004), pp. 17-51.

[21] S. Adly, D. Goeleven, And B.-K. Le, Stability analysis and attractivity results of a DC-DC buck converter, Set Valued and Variational Analysis, 20 (2012), pp. 331-353.

[22] S. Adly, D. Goeleven, And M. ThérA, Periodic solutions of evolution variational inequalities-a method of guiding functions, Chinese Annals of Mathematics, Series B, 30 (2009), pp. 261-272.

[23] S. Adly AND T. HAdDAD, An implicit sweeping process approach to quasistatic evolution variational inequalities, SIAM Journal on Mathematical Analysis, 50 (2018), pp. 761778.

[24] S. Adly AND T. HAdDAD, On evolution quasi-variational inequalities and implicit statedependent sweeping processes, Discrete and Continuous Dynamical Systems Series S, (2019). DoI: 10.3934/dcdss.2020105.

[25] S. Adly AND T. HADDAD, Well-posedness of nonconvex degenerate sweeping process via unconstrained evolution problems, Nonlinear Analysis: Hybrid Systems, 36 (2020). paper 100832.

[26] S. Adly, T. Haddad, AND B.-K. LE, State-dependent implicit sweeping process in the framework of quasistatic evolution quasi-variational inequalities, Journal of Optimization Theory and Applications, 182 (2019), pp. 473-493.

[27] S. Adly, T. HADdad, AND L. Thibault, Convex sweeping process in the framework of measure differential inclusions and evolution variational inequalities, Mathematical Programming B, 148 (2014), pp. 5-47.

[28] S. Adly, A. Hantoute, And B.-K. Le, Nonsmooth Lur'e dynamical systems in Hilbert spaces, Set-Valued and Variational Analysis, 24 (2016), pp. 13-35.

[29] S. Adly, A. Hantoute, AND B.-K. Le, Maximal monotonicity and cyclic-monotonicity arising in nonsmooth Lur'e dynamical systems, Journal of Mathematical Analysis and Applications, 448 (2017), pp. 691-706.

[30] S. Adly, A. Hantoute, And B. NGuyen, Invariant sets and Lyapunov pairs for differential inclusions with maximal monotone operators, Journal of Mathematical Analysis and Applications, 457 (2018), pp. 1017-1037.

[31] S. Adly, A. Hantoute, and B.-T. NGUyen, Equivalence between differential inclusions involving prox-regular sets and maximal monotone operators. arXiv:1704.04913v2, 2018.

[32] S. Adly, A. Hantoute, And B.-T. NGUYen, Lyapunov stability of differential inclusions with Lipschitz Cusco perturbations of maximal monotone operators, Set-Valued and Variational Analysis, (2018). https://doi.org/10.1007/s11228-019-00513-4.

[33] S. Adly, A. Hantoute, and B.-T. NGuyen, Lyapunov stability of differential inclusions involving prox-regular sets via maximal monotone operators, Journal of Optimization Theory and Applications, 182 (2019), pp. 906-934.

[34] S. Adly, A. Hantoute, And M. ThÉRA, Nonsmooth Lyapunov pairs for infinite-dimensional first-order differential inclusions, Nonlinear Analysis, 75 (2012), pp. 985-1008.

[35] S. Adly, A. Hantoute, AND M. ThÉRA, Nonsmooth Lyapunov pairs for differential inclusions governed by operators with nonempty interior domain, Mathematical Programming, 157 (2016), pp. 349-374.

[36] S. AdLy AND B.-K. LE, Stability and invariance results for a class of non-monotone set-valued Lur'e dynamical systems, Applicable Analysis, 5 (2014), pp. 1087-1105.

[37] S. AdLY AND B.-K. LE, Unbounded second-order state-dependent Moreau's sweeping processes in Hilbert spaces, Journal of Optimization Theory and Applications, 169 (2016), pp. 407423.

[38] S. Adly And B.-K. Le, Non-convex sweeping processes involving maximal monotone operators, Optimization, 66 (2017), pp. 1465-1486.

[39] S. Adly AND B.-K. Le, On semicoercive sweeping process with velocity constraint, Optimization Letters, 12 (2018), pp. 831-843. 
[40] S. AdLy AND F. NACRY, An existence result for discontinuous second-order nonconvex statedependent sweeping processes, Applied Mathematics and Optimization, (2019).

[41] S. Adly, F. Nacry, And L. ThiBault, Preservation of prox-regularity of sets and application to constrained optimization, SIAM Journal on Optimization, 26 (2016), pp. 448-473.

[42] S. Adly, F. Nacry, And L. Thibault, Discontinuous sweeping process with prox-regular sets, ESAIM: Control, Optimisation and Calculus of Variations, 23 (2017), pp. 1293-1329.

[43] D. Affane And M.-F. Yarou, Unbouded perturbation for a class of variational inequalities, Discussiones Mathematicae, Differential Inclusions, Control and Optimization, 37 (2017), pp. 83-99.

[44] M.-A. Aizerman And E.-S. Pyatnitskit, Foundation of a theory of discontinuous systems I, Automation and Remote Control (Avtom. Telemekh.), 7 (1974), pp. 33-47.

[45] M.-A. Aizerman And E.-S. Pyatnitskil, Foundation of a theory of discontinuous systems II, Automation and Remote Control (Avtom. Telemekh.), 8 (1974), pp. 39-61.

[46] F. Aliouane, D. Azzam-Laouir, C. Castaing, and M. Monteiro-Marques, Second-order and state-dependent sweeping process in Hilbert space, Journal of Optimization Theory and Applications, 182 (2019), pp. 153-188.

[47] F. Aliouane And F. Azzam-LaOuir, Second order sweeping process with a Lipschitz perturbation, Journal of Mathematical Analysis and Applications, 452 (2017), pp. 729-746.

[48] F. Alvarez, On the minimizing property of a second order dissipative system in Hilbert spaces, SIAM Journal on Control and Optimization, 38 (2000), pp. 1102-1119.

[49] F. Alvarez and H. Attouch, An inertial proximal method for maximal monotone operators via discretization of a nonlinear oscillator with damping, Set-Valued Analysis, 9 (2001), pp. 3-11.

[50] J. Alvarez, I. Orlov, , AND L. ACHO, An invariance principle for discontinuous dynamic systems with applications to a Coulomb friction oscillator, Transactions ASME J. Dynam. Systems Measurement Control, 122 (2000), pp. 687-690.

[51] N.-R. Amundson, A. Caboussat, J. he, C. Landry, and J.-H. Seinfled, A dynamic optimization problem related to organic aerosols, C.R. Acad. Scio. paris Ser. I, 344 (2007), pp. 519-522.

[52] B.-D.-O. Anderson And P.-J. Moylan, Synthesis of linear time-varying passive networks, IEEE Transactions on Circuits and Systems, 21 (1974), pp. 678-687.

[53] A.-S. Antipin, Minimization of convex functions on convex sets by means of differential equations, Russian Differentsial'nye Uravneniya, 30 (1994), pp. 1475-1486. Translated in Differential Equations 30(9), 1365-1375, 1994.

[54] C.-E. Arroud And G. Colombo, A maximum principle for the controlled sweeping process, Set-Valued Var. Anal., 26 (2018), pp. 607-629.

[55] H. Attouch, A. CABot, AND M. CZARnecki, Asymptotic behavior of nonautonomous monotone and subgradient evolution equations, Transactions of the American Mathematical Society, 370 (2018), pp. 755-790.

[56] H. Attouch And M.-O. Czarnecki, Asymptotic behaviour of coupled dynamical systems with multiscale aspects, J. of Differential Equations, 248 (2010), pp. 1315-1344.

[57] H. Attouch And J. Peypouquet, Convergence of inertial dynamics and proximal algorithms governed by maximally monotone operators, Mathematical Programming series B, 174 (2019).

[58] H. Attouch, J. Peypouquet, And P. Redont, A dynamical approach to an inertial forwardbackward algorithm for convex minimization, SIAM Journal on Optimization, 24 (2014), pp. 232-256.

[59] H. Attouch, J. Peypouquet, And P. Redont, Fast convex optimization via inertial dynamics with Hessian driven damping, Journal of Differential Equations, 261 (2016), pp. 57345783.

[60] H. Attouch And B.-F. Svaiter, A continuous dynamical Newton-like approach to solving monotone inclusions, SIAM Journal on Control and Optimization, 49 (2011), pp. 574598.

[61] J.-P. Aubin, Viability Theory, Systems and Control: Foundations and Applications, Springer Science Business Media, 1991.

[62] J.-P. Aubin And A. Cellina, Differential Inclusions: Set-Valued Maps and Viability, Springer Verlag, Berlin, 1984.

[63] J.-P. Aubin and H. Frankowska, Set-Valued Analysis, Birkhäuser, Boston Basel Berlin, 1990.

[64] D. Azzam-Laouir, W. Belhoula, C. Castaing, and M. Monteiro-Marques, Multi-valued perturbation to evolution problems involving time dependent maximal monotone operators, Evolution Equations and Control Theory, 9 (2020), pp. 219-254. 
[65] D. Azzam-Laouir, C. Castaing, and M.-D.-P. Monteiro-Marques, Perturbed evolution problems with continuous bounded variation in time and applications, Set-Valued Var. Anal., 26 (2018), pp. 693-728.

[66] D. Azzam-Laouir and S. IzzA, Existence of solutions for second-order perturbed nonconvex sweeping process, Computers in Mathematics with Applications, 62 (2011), pp. 17361744 .

[67] D. Azzam-Laouir, S. Izza, and L. Thibault, Mixed semicontinuous perturbation of nonconvex state-dependent sweeping process, Set-Valued and Variational Analysis, 22 (2017), pp. 271-283.

[68] V.-I. BABitsky, Theory of Vibro-Impact Systems and Applications, Foundations of Engineering Mechanics, Springer-Verlag, Berlin Heidelberg, 1998.

[69] A. Bacciotti, F. Ceragioli, And L. Mazzi, Differential inclusions and monotonicity conditions for nonsmooth Liapunov functions, Set-Valued Analysis, 8 (2000), pp. 299-309.

[70] A. Bacciotti and L. Rosier, Liapunov Functions and Stability in Control Theory, Communications and Control Engineering, Springer Verlag, Berlin Heidelberg, 2nd ed., 2005.

[71] F. Bagagiolo, Dynamic programming for some optimal control problems with hysteresis, Nonlinear Differential Equations and Applications NoDEA, 9 (2002), pp. 149-174.

[72] B. BAJI AND A. САВOT, An inertial proximal algorithm with dry friction: finite convergence results, Set Valued Analysis, 14 (2006), pp. 1-23.

[73] P. BALlaRD, The dynamics of discrete mechanical systems with perfect unilateral constraints, Archive for Rational Mechanics and Analysis, 154 (2000), pp. 199-274.

[74] P. Ballard, Formulation and well-posedness of the dynamics of rigid body systems with perfect unilateral constraints, Phil. Trans. R. Soc. Lond. A, 359 (2001), pp. 2327-2346.

[75] P. BALlard AND S. BASSEville, Existence and uniqueness for dynamical unilateral contact with Coulomb friction: a model problem, ESAIM: Mathematical Modelling and Numerical Analysis (M2AN), 39 (2005), pp. 59-77.

[76] P. Ballard, A. LÉGer, And E. Pratt, Stability of discrete systems involving shocks and friction, in Analysis and Simulation of Contact Problems, P. Wriggers and U. Nackenhorst, eds., Lecture Notes in Applied and Computational Mechanics, Springer Verlag, 2006, pp. 343-350.

[77] X. BAN, H.-X. LiU, M.-C. FERris, AND B. RAN, A link-node complementarity model and solution algorithm for dynamic user equilibria with exact propagations, Transportation Research Part B, 42 (2008), pp. 823-842.

[78] X. BAn, J.-S. PANG, H.-X. LIU, AND R. MA, Continuous-time point-queue models in dynamic network loading, Transportation Research Part B, 46 (2012), pp. 360-380.

[79] X. BAN, J.-S. PANG, H.-X. LiU, AND R. MA, Modeling and solving continuous-time instantaneous dynamic user equilibria: A differential complementarity systems approach, Transportation Research Part B, 46 (2012), pp. 389-408.

[80] N.-A. Barabanov and V.-A. Yakubovich, Absolute stability of control systems with one hysteresis nonlinearity, Automation and Remote Control, 12 (1979), pp. 5-12.

[81] P.-I. Barton, K.-A. Khan, P. Stechlinski, And H.-J. Watson, Computationally relevant generalized derivatives: theory, evaluation and applications, Optimization Methods and Software, 33 (2018), pp. 1030-1072.

[82] S. BASSEVIlle AND A. LÉGER, Stability of equilibrium states in a simple system with unilateral contact and Coulomb friction, Archive of Applied Mechanics, 76 (2006), pp. 403-428.

[83] S. Basseville, A. LÉGer, And E. Pratt, Investigation of the equilibrium states and their stability for a simple model with unilateral contact and Coulomb friction, Archive of Applied Mechanics, 73 (2003), pp. 409-420.

[84] J. BASTIEn, Convergence order of implict Euler numerical scheme for maximal monotone differential inclusions, Z. Angew. Math. Phys., 64 (2013), pp. 955-966.

[85] J. BASTIEN, Study of a driven and braked wheel using maximal monotone differential inclusions: applications to the nonlinear dynamics of wheeled vehicles, Archive of Applied Mechanics, 84 (2014), pp. 851-880.

[86] J. Bastien, F. Bernardin, and C.-H. Lamarque, Systèmes Dynamiques Discrets Non Réguliers Déterministes ou Stochastiques, Lavoisier, Paris, France, 2012.

[87] J. Bastien and C. Lamarque, Persoz's gephyroidal model described by a maximal monotone differential inclusion, Archive of Applied Mechanics, 78 (2008), pp. 393-407.

[88] J. Bastien and C.-H. Lamarque, Maximal monotone model with history term, Nonlinear Analysis, 63 (2005), pp. e199-e207.

[89] J. Bastien and C.-H. Lamarque, Theoretical study of a chain sliding on a fixed support, Mathematical Problems in Engineering, (2009), pp. 1-19. Article ID 361296.

[90] J. Bastien, G. Michon, L. Manin, And R. Dufour, An analysis of the modified Dahl and 
Masing models: application to a belt tensioner, Journal of Sound and Vibration, 302 (2007), pp. 841-864.

[91] J. Bastien, M. Schatzman, And C.-H. Lamarque, Study of some rheological models with a finite number of degrees of freedom, European Journal of Mechanics A/Solids, 19 (2000), pp. 277-307.

[92] J. Bastien, M. Schatzman, And C.-H. Lamarque, Study of an elastoplastic model with an infinite number of internal degrees of freedom, European Journal of Mechanics A/Solids, 21 (2002), pp. 199-222.

[93] C. Batlle, E. Fossas, I. Merillas, and A. Miralles, Generalized discontinuous conduction modes in the complementarity formalism, IEEE Transactions on Circuits and Systems-II: Express Briefs, 52 (2005), pp. 447-451.

[94] M. Baumann, J.-J.-B. Biemond, R.-I. Leine, And N. van DE Wouw, Synchronization of impacting systems with a single constraint, Physica D, 362 (2018), pp. 9-23.

[95] M. Baumann And R.-I. Leine, A synchronization-based state observer for impact oscillators using only collision time information, Int. Journal of Robust and Nonlinear Control, 26 (2016), pp. 2542-2563.

[96] B.-T. BAUMRUCKer AND L.-T. BIEGLER, MPEC strategies for optimization of a class of hybrid dynamic systems, Journal of Process Control, 19 (2009), pp. 1248-1256.

[97] H.-H. Bauschke and P.-L. Combettes, Convex Analysis and Monotone Operator Theory in Hilbert Spaces, Canadian Mathematical Society Books in Mathematics, Springer, 2011.

[98] C. BeHn, Adaptive control of straight worms without derivative measurement, Multibody System Dynamics, 26 (2011), pp. 213-243.

[99] H. Benabdellah, Existence of solutions to the nonconvex sweeping process, Journal of Differential Equations, 164 (2000), pp. 286-295.

[100] H. Benabdellah And A. Faik, Perturbations convexes et non convexes des équations d'évolution, Portugaliae Mathematica, 53 (1996), pp. 187-208.

[101] A. Benveniste, T. Bourke, B. Caillaud, and M. Pouzet, Non-standard semantics of hybrid systems modelers, Journal of Computer and System Sciences, (2012).

[102] F. Bernard And L. Thibault, Uniform prox-regularity of functions and epigraphs in Hilbert spaces, Nonlinear Analysis: Theory, Methods and Applications, 60 (2005), pp. 187-207.

[103] F. BERnicot AND J. Venel, Stochastic perturbation of sweeping process and a convergence result for an associated numerical scheme, Journal of Differential Equations, 251 (2011), pp. 1195-1224.

[104] D.-S. Bernstein, Matrix Mathematics. Theory, facts, and Formulas with Application to Linear Systems Theory, Princeton University Press, Princeton, NJ, USA, 2005.

[105] P. Bettiol, A deterministic approach to the Skorokhod problem, Control and Cybernetics, 35 (2006), pp. 787-801.

[106] W. BIAN AND X. XUE, Evolution differential inclusion with projection for solving constrained nonsmooth convex optimization in Hilbert space, Set-Valued Var. Anal., 20 (2012), pp. 203-227.

[107] J.-J.-B. Biemond, W. Michiels, AND N. VAn DE Wouw, Stability analysis of equilibria of linear delay complementarity systems, IEEE Control Systems Letters, 1 (2017), pp. 158163.

[108] A. Bisoffi, M. D. Lio, A.-R. Teel, And L. Zaccarian, Global asymptotic stability of a PID control system with Coulomb friction, IEEE Transactions on Automatic Control, 63 (2018), pp. 2654-2661.

[109] A. Blumentals, B. Brogliato, and F. Bertalls-Descoubes, The contact problem in Lagrangian systems subject to bilateral and unilateral constraints, with or without sliding Coulomb's friction: A tutorial, Multibody System Dynamics, 38 (2016), pp. 43-76.

[110] I. BoIKO, Oscillations and transfer properties of relay servo systems-the locus of a perturbed relay system approach, Automatica, 41 (2005), pp. 677-683.

[111] J. BolTE, Continuous gradient projection method in Hilbert spaces, Journal of Optimizaton Theory and Applications, 119 (2003), pp. 235-259.

[112] R.-I. Bot And E.-R. Csetnek, Approaching nonsmooth nonconvex optimization problems thourgh first order dynamical systems with hidden acceleration and Hessian damping terms, Set-Valued Var. Anal., 26 (2018), pp. 227-245.

[113] M. Bounkhel, Existence results for nonconvex differential inclusions, Portugaliae Matematica, 59 (2002), pp. 283-309.

[114] M. BounKHEL, Existence and uniqueness of some variants of nonconvex sweeping processes, Journal of Nonlinear and Convex Analysis, 8 (2007), pp. 311-323.

[115] M. Bounkhel And D. Azzam-Laouir, Existence results on the second-order nonconvex sweeping processes with perturbations, Set-Valued Var. Anal., 12 (2004), pp. 291-318. 
[116] M. Bounkhel And L. ThiBault, Nonconcex sweeping process and prox-regularity in Hilbert space, Journal of Nonlinear and Convex Analysis, 6 (2001), pp. 359-374.

[117] M. Bounkhel And M. Yarou, Existence results for first and second order nonconvex sweeping process with delay, Portugaliae Mathematica, 61 (2004), pp. 207-230.

[118] J.-M. Bourgeot And B. BRogliato, Tracking control of Lagrangian complementarity systems, International Journal of Bifurcation and Chaos, 15 (2005), pp. 1839-1866.

[119] Y. Brenier, W. Gangbo, G. Savaré, and M. Westdickenberg, Sticky particle dynamics with interactions, Journal de Mathématiques Pures et Appliquées, 99 (2013), pp. 577-617.

[120] A. Bressan, Incompatbilita dei teoremi di esistenza e di unicita del moto per un tipo molto comune e regolare di sistemi meccanici, Annali della Scuola Normale Superiore di Pisa, Classe di Scienze 3e série, 14 (1959), pp. 333-348.

[121] A. Bressan, A. Cellina, And G. Colombo, Upper semicontinuous differential inclusions without convexity, Proceedings of the American Mathematcal Society, 186 (1989), pp. $771-775$.

[122] A. Bressan, M. Mazzola, and K. NGuyen, Approximation of sweeping processes and controllability for a set-valued evolution, SIAM Journal on Control and Optimization, 57 (2019), pp. 2487-2514.

[123] H. Brezis, Opérateurs Maximaux Monotones et Semi-Groupes de Contraction dans les Espaces de Hilbert, North Holland, Amsterdam, 1973.

[124] L.-M. Briceno-Arias, N.-D. HoAng, And J. Peypouquet, Existence, stability and optimality for optimal control problems governed by maximal monotone operators, Journal of Differential Equations, 260 (2016), pp. 733-757.

[125] B. Brogliato, Some perspectives on the analysis and control of complementarity systems, IEEE Transactions on Automatic Control, 48 (2003), pp. 918-935.

[126] B. Brogliato, Absolute stability and the Lagrange-Dirichlet theorem with monotone multivalued mappings, Systems and Control Letters, 51 (2004), pp. 343-353.

[127] B. Brogliato, Some results on the controllability of planar evolution variational inequalities, Systems and Control Letters, 54 (2005), pp. 65-71.

[128] B. BROGLiAto, Some results on the optimal control with unilateral state constraints, Nonlinear Analysis: Theory, Methods and Applications, 70 (2009), pp. 3626-3657.

[129] B. Brogliato, Inertial couplings between unilateral and bilateral holonomic constraints in frictionless Lagrangian systems, Multibody System Dynamics, 29 (2013), pp. 289-325.

[130] B. Brogliato, Nonsmooth Mechanics. Models, Dynamics and Control, Springer International Publishing Switzerland, 3rd ed., 2016. Erratum/addendum at https://hal.inria.fr/hal01331565.

[131] B. Brogliato, Feedback control of multibody systems with joint clearance and dynamic backlash: a tutorial, Multibody System Dynamics, 42 (2018), pp. 283-315.

[132] B. Brogliato, Non-autonomous higher-order Moreau's sweeping process: Well-posedness, stability and Zeno trajectories, European Journal of Applied Mathematics, 29 (2018), pp. 941-968. Extended version at https://hal.inria.fr/hal-01722772.

[133] B. Brogliato, A. Danillidis, C. Lemaréchal, and V. ACAry, On the equivalence between complementarity systems, projected systems and differential inclusions, Systems and Control Letters, 55 (2006), pp. 45-51.

[134] B. Brogliato and D. Goeleven, The Krasovskii-LaSalle invariance principle for a class of unilateral dynamical systems, Mathematics of Control, Signals and Systems, 17 (2005), pp. $57-76$.

[135] B. Brogliato and D. Goeleven, Well-posedness, stability and invariance results for a class of multivalued Lur'e dynamical systems, Nonlinear Analysis: Theory, Methods and Applications, 74 (2011), pp. 195-212.

[136] B. Brogliato And D. Goeleven, Existence, uniqueness of solutions and stability of nonsmooth multivalued Lur'e dynamical systems, Journal of Convex Analysis, 20 (2013), pp. 881-900.

[137] B. Brogliato And D. Goeleven, Singular mass matrix and redundant constraints in unilaterally constrained Lagrangian and Hamiltonian systems, Multibody System Dynamics, 35 (2015), pp. 39-61.

[138] B. Brogliato AND W.-P.-M.-H. HeEmels, Observer design for Lur'e systems with multivalued mappings: a passivity approach, IEEE Transactions on Automatic Control, 54 (2009), pp. 1996-2001.

[139] B. Brogliato, R. Lozano, B. Maschke, and O. Egeland, Dissipative Systems Analysis and Control, Communications and Control Engineering, Springer Nature Switzerland AG, London, third ed., 2020.

[140] B. Brogliato, M. Mabrouk, and A. Z. Rio, On the controllability of linear juggling me- 
chanical systems, Systems and Control Letters, 55 (2006), pp. 350-367.

[141] B. Brogliato, S.-I. Niculescu, and M.-D.-P. Monteiro-Marques, On tracking control of a class of complementary-slackness hybrid mechanical systems, Systems and Control Letters, 39 (2000), pp. 255-266.

[142] B. Brogliato, S.-I. Niculescu, and P. Orhant, On the control of finite-dimensional mechanical systems with unilateral constraints, IEEE Transactions on Automatic Control, 42 (1997), pp. 200-215.

[143] B. Brogliato And L. ThiBault, Existence and uniqueness of solutions for non-autonomous complementarity dynamical systems, Journal of Convex Analysis, 17 (2010), pp. 961-990.

[144] M. Brokate AND P. KReJCI, Optimal control of ODE systems involving a rate independent variational inequality, Discrete and Continuous Dynamical Systems - Series B, 18 (2013), pp. 331-348.

[145] C.-I. Byrnes And A. Isidori, Asymptotic stabilization of minimum phase nonlinear systems, IEEE Transactions on Automatic Control, 36 (1991), pp. 1122-1337.

[146] A. САвот, Stabilization of oscillators subject to dry friction: finite time convergence versus exponential decay results, Transactions of the American Mathematical Society, 360 (2008), pp. 103-121.

[147] A. CABOt And L. PaOli, Asymptotics for some vibro-impact problem with linear dissipation term, Journal de Mathématiques Pures et Appliquées, 87 (2007), pp. 291-323.

[148] A. CABoussat And C. LANDRY, A second order scheme for solving optimization-constrained differential equations with discontinuities, in Proceedings of ENUMATH07, the 7th European Conference on Numerical Mathematics and Advanced Applications, Graz, Austria, 2008, Springer Verlag, pp. 761-768.

[149] M.-K. Camlibel, Complementarity Methods in the Analysis of Piecewise Linear Dynamical Systems, PhD thesis, Katholieke Universiteit Brabant, The Netherlands, 2001. ISBN 90 $5668073 \mathrm{X}$.

[150] M.-K. Camlibel, Popov-Belevitch-Hautus type tests for the controllability of linear complementarity systems, Systems and Control Letters, 56 (2007), pp. 381-387.

[151] M.-K. Camlibel, W.-P.-M.-H. Heemels, and J.-M. Schumacher, On linear passive complementarity systems, European Journal of Control, 8 (2002), pp. 220-237.

[152] M.-K. Camlibel, W.-P.-M.-H. Heemels, A.-J. van der Schaft, and J.-M. Schumacher, Switched networks and complementarity, IEEE Transactions on Circuits and Systems I, 50 (2003), pp. 1036-1048.

[153] M.-K. Camlibel, L. Iannelli, and A. TAnwani, Convergence of proximal solutions for evolution inclusions with time-dependent maximal monotone operators. https://hal. archives-ouvertes.fr/hal-02144478/, 2019.

[154] M.-K. Camlibel, L. IAnnelli, A. Tanwani, and S. Trenn, Differential-algebraic inclusions with maximal monotone operators, in 55th IEEE Conference on Decision and Control, Las Vegas, USA, December 2016, pp. 610-615.

[155] M.-K. Camlibel, L. Iannelli, and F. Vasca, Passivity and complementarity, Mathematical Programming A, 145 (2014), pp. 531-563.

[156] M.-K. Camlibel, J.-S. Pang, and J. Shen, Conewise linear systems: non-Zenoness and observability, SIAM Journal on Optimization, 17 (2006), pp. 1056-1101.

[157] M.-K. Camlibel, J.-S. Pang, and J. Shen, Lyapunov stability of complementarity and extended systems, SIAM Journal on Optimization, 17 (2006), pp. 1056-1101.

[158] M.-K. Camlibel and J.-M. Schumacher, Copositive Lyapunov functions, in Unsolved Problems in Mathematical Systems and Control Theory, Princeton Univ. Press, 2004, pp. 189193.

[159] M.-K. Camlibel And J.-M. Schumacher, Linear passive systems and maximal monotone mappings, Mathematical Programming B, 157 (2016), pp. 397-420.

[160] T.-H. CAO And B.-S. Mordukhovich, Optimal control of a perturbed sweeping process via discrete approximations,, Disc. Cont. Dyn. Syst. Ser. B, 21 (2016), pp. 3331-3358.

[161] T.-H. CAO AND B.-S. Mordukhovich, Optimal control of a nonconvex perturbed sweeping process, arXiv:1711.02267v1, (2017).

[162] T.-H. CAO AND B.-S. MordukHOVICH, Optimality conditions for a controlled sweeping process with applications to the crowd motion model, Disc. Cont. Dyn. Syst. Ser. B, 22 (2017), pp. 267-306.

[163] S. Carl, V.-K. Le, And D. Motreanu, Nonsmooth Variational Problems and Their Inequalities. Comparison Principles and Applications, Springer Monographs in Mathematics, Springer Science+Business Media, New York, USA, 2007.

[164] A. Carta, M. Chaves, And J.-L. Gouzé, Continuous-switch piecewise quadratic models of biological networks: Application to bacterial growth, Automatica, 61 (2015), pp. 164-172. 
[165] R. CASey, H. D. Jong, And J.-L. Gouzé, Piecewise-linear models of genetic regulatory networks: Equilibria and their stability, Journal of Mathematical Biology, 52 (2006), pp. $27-56$.

[166] C. Castaing, Version aléatoire de problème de rafle par un convexe variable, C.R. Acad. Sci. Paris, Série A, 277 (1973), pp. 1057-1059.

[167] C. Castaing, Equations différentielles. Rafle par un convexe aléatoire à variation continue à droite, C.R. Acad. Sci. Paris, Série A, 282 (1976), pp. 515-518.

[168] C. Castaing, Quelques problèmes d'évolution du second ordre, in Séminaire d'Analyse Convexe, 1988.

[169] C. Castaing, T.-X.-D. HA, And M. VAladier, Evolution equations governed by the sweeping process, Set-Valued Analysis, 1 (1993), pp. 109-139.

[170] C. Castaing, A.-G. Ibrahim, and M. Yarou, Some contributions to nonconvex sweeping process, Journal of Nonlinear and Convex Analysis, 10 (2009), pp. 1-20.

[171] C. Castaing, A.-G. Ibrahim, and M.-F. Yarou, Existence problems in second order evolution inclusions: discretization and variational approach, Taiwanese Journal of Mathematics, 12 (2008), pp. 1435-1477.

[172] C. Castaing and M.-D.-P. Monteiro-Marques, BV periodic solutions of an evolution problem associated with continuous moving convex sets, Set-Valued Analysis, 3 (1995), pp. 381-399.

[173] C. Castaing and M.-D.-P. Monteiro-Marques, Periodic solutions of an evolution problems associated with a moving convex sets, C.R. Acad.Sci. Paris Ser. A, 321 (1995), pp. 531536.

[174] C. Castaing and M.-D.-P. Monteiro-Marques, Topological properties of solution sets for sweeping processes with delay, Portugaliae Mathematica, 54 (1997), pp. 485-507.

[175] C. Castaing, M.-D.-P. Monteiro-Marques, and P. R. De Fitte, Some problems in optimal control governed by the sweeping process, Journal of Nonlinear and Convex Analysis, 15 (2014), pp. 1043-1070.

[176] C. Castaing, M.-D.-P. Monteiro-Marques, and P. R. De Fitte, A Skorokhod problem governed by a closed convex moving set, Journal of Convex Analysis, 23 (2016), pp. 387423.

[177] C. Castaing, A. Salvadori, and L. Thibault, Functional evolution equations governed by nonconvex sweeping process, Journal of Nonlinear and Convex Analysis, 2 (2001), pp. $217-241$.

[178] E. Cavazzuti, M. Pappalardo, and M. Passacantando, Nash equilibria, variational inequalities, and dynamical systems, Journal of Optimization Theory and Applications, 114 (2002), pp. 491-506.

[179] A. Cellina AND V. STAICU, On evolution equations having monotonicities of opposite sign, Journal of Differential Equations, 90 (1991), pp. 71-80.

[180] F. Ceragioli, C. De Persis, and P. Frasca, Discontinuities and hysteresis in quantized average consensus, Automatica, 47 (2011), pp. 1916-1928.

[181] F. Ceragioli And P. Frasca, Consensus and disagreement: The role of quantized behaviors in opinion dynamics, SIAM Journal on Control and Optimization, 56 (2018), pp. 10561080 .

[182] A. Champneys and P. Varkonyi, The Painlevé paradox in contact mechanics, IMA Journal of Applied Mathematics, 81 (2016), pp. 538-588.

[183] P. Champsaur, J. Drèze, And C. Henry, Stability theorems with economic applications, Econometrica, 45 (1977), pp. 273-294.

[184] X. Chateau And Q.-S. NGUYen, Buckling of elastic structures in unilateral contact, European Journal of Mechanics, A/Solids, 10 (1991), pp. 71-89.

[185] M. Chaves AND J.-L. GouzÉ, Exact control of genetic networks in a qualitative framework: The bistable switch example, Automatica, 47 (2011), pp. 1105-1112.

[186] N. Chemetov and M.-D.-P. Monteiro-Marques, Non-convex quasi-variational differential inclusions, Set-Valued and Variational Analysis, 15 (2007), pp. 209-221.

[187] D. Chen, G. YANG, AND Z. HAN, Impulsive observer for input-to-state stability based synchronization of Lur'e differential inclusion system, Communications in Nonlinear Science and Numerical Simulation, 17 (2012), pp. 2990-2996.

[188] X. Chen and Z. WAng, Differential variational inequality approach to dynamic games with shared constraints, Mathematical Programming A, 146 (2014), pp. 379-408.

[189] A. Cherukuri, E. Mallada, and J. Cortès, Asymptotic convergence of constrained primaldual dynamics, Systems and Control Letters, 87 (2016), pp. 10-15.

[190] A. Cherukuri, E. Mallada, S. Low, and J. Cortès, The role of convexity in saddle-point dynamics: Lyapunov function and robustness, IEEE Transactions on Automatic Control, 
63 (2018), pp. 2449-2464.

[191] F.-H. Clarke, Y.-S. Ledyaev, R.-J. Stern, and P.-R. Wolenski, Nonsmooth Analysis and Control Theory, Springer, New York, 1998.

[192] M.-G. Cojocaru, Monotonicity and existence of periodic orbits for projected dynamical systems on Hilbert spaces, Proceedings of the American Mathematical Society, 134 (2005), pp. 793-804.

[193] M.-G. Cojocaru, Dynamic equilibria of group vaccination strategies in a heterogeneous population, J. Global Optimization, 40 (2008), pp. 51-63.

[194] M.-G. Cojocaru, C.-T. Bauch, And M.-D. Johnston, Dynamics of vaccination strategies via projected dynamical systems, Bulletin of Mathematical Biology, 69 (2007), pp. 14531476 .

[195] M.-G. Cojocaru, P. Daniele, and A. Nagurney, Projected dynamical systems and evolutionary variational inequalities via Hilbert spaces with applications, Journal of Optimization Theory and Applications, 127 (2005), pp. 549-563.

[196] M.-G. Cojocaru, P. Daniele, And A. Nagurney, Double-layered dynamics: A unified theory of projected dynamical systems and evolutionary variational inequalities, European Journal of Operational Research, 175 (2006), pp. 494-507.

[197] M.-G. Cojocaru AND L.-B. Jonker, Existence of solutions to projected differential equations in Hilbert spaces, Proceedings of the American Mathematical Society, 132 (2004), pp. 183193.

[198] P. Colli, P. Krejci, E. Rocca, And J. Sprekels, Nonlinear evolution inclusions arising from phase change models, Czechoslovak Mathematical Journal, 57 (2007), pp. 1067-1098.

[199] G. Colombo and V.-V. Goncharov, The sweeping process without convexity, Set-Valued Analysis, 7 (1999), pp. 357-374.

[200] G. Colombo, R. Henrion, D.-H. Hoang, and S.-B. Mordukhovich, Optimal control of the sweeping process, Dyn. Contin. Discrete Impuls. Syst. Ser. B Appl. Algorithms, 19 (2012), pp. 117-159.

[201] G. Colombo, R. Henrion, D.-H. Hoang, and S.-B. Mordukhovich, Discrete approximations of a controlled sweeping process, Set-Valued and Variational Analysis, 23 (2015), pp. 69-86.

[202] G. Colombo, R. Henrion, D.-H. Hoang, and S.-B. Mordukhovich, Optimal control of the sweeping process over polyhedral controlled sets, Journal of Differential Equations, 260 (2016), pp. 3397-3447.

[203] G. Colombo and C. Kozaily, Existence and uniqueness of solutions for an integral perturbation of Moreau's sweeping process, Journal of Convex Analysis, 27 (2020).

[204] G. Colombo and M.-D.-P. Monteiro-Marques, Sweeping by a continuous prox-regular set, Journal of Differential Equations, 187 (2003), pp. 46-62.

[205] G. Colombo, B. Mordukhovich, and D. NGuyen, Optimal control of sweeping processes in robotics and traffic flow models, Journal of Optimization Theory and Applications, 182 (2019), pp. 439-472.

[206] G. Colombo, B.-S. Mordukhovich, And D. Nguyen, Optimization of a perturbed sweeping process by constrained discontinuous controls, SIAM Journal on Control and Optimization, (2020).

[207] G. Colombo and M. Palladino, The minimum time function for the controlled Moreau's sweeping process, SIAM Journal on Control and Optimization, 54 (2016), pp. 2036-2052.

[208] P.-L. Combettes And J.-C. Pesquet, Fixed-Point Algorithms for Inverse Problems in Science and Engineering, vol. 49 of Springer Optimization and Its Applications, Springer, New York, 2011, ch. Proximal splitting methods in signal processing, pp. 185-212.

[209] B. CoRnet, Existence of slow solutions for a class of differential inclusions, Journal of Mathematical Analysis and Applications, 96 (1983), pp. 130-147.

[210] R.-W. Cottle, J.-S. Pang, and R.-E. Stone, The Linear Complementarity Problem, Computer Science and Scientific Computing, Academic Press, 1992.

[211] P. Daniele, Innovations in Financial and Economic Networks, New Dimensions in Networks, Edward Elgar Publishing, 2003, ch. Variational inequalities for evolutionary financial equilibrium, pp. 84-108.

[212] P. Daniele, Dynamic Networks and Evolutionary Variational Inequalities, New Dimensions in Networks, Edward Elgar Publishing, 2006.

[213] P. Daniele, A. Maugeri, And W. Oettli, Variational inequalities and time-dependent traffic equilibria, Comptes Rendus Académie des Sciences Paris, 326 (1998), pp. 1059-1062.

[214] P. Daniele, A. Maugeri, And W. Oettli, Time-dependent variational inequalities, Journal of Optimization Theory and Applications, 104 (1999), pp. 543-555.

[215] J.-C.-A. de Bruin, A. Doris, N. van de Wouw, W.-P.-M.-H. Heemels, And H. NiJmeiJer, 
Control of mechanical motion systems with non-collocation of actuation and friction: a Popov criterion approach for input-to-state stability and set-valued nonlinearities, Automatica, 45 (2009), pp. 405-415.

[216] H. de Jong, J.-L. Gouzé, C. Hernandez, M. Page, T. Sari, and J. Geiselmann, Qualitative simulation of genetic regulatory networks using piecewise-linear models, Bulletin of Mathematical Biology, 66 (2004), pp. 301-340.

[217] M. De Pinho, M. Ferreira, And G. Smirnov, Optimal control involving sweeping processes, Set-Valued and Variational Analysis, 27 (2019), pp. 523-548.

[218] G. De Sandre, M. Forti, P. Nistri, And A. Premoli, Dynamical analysis of full-range cellular neural networks by exploiting differential variational inequalities, IEEE Transactions on Circuits and Systems-I: Regular Papers, 54 (2007), pp. 1736-1749.

[219] G. DE SAXCÉ AND Z.-Q. Feng, The bipotential method: a constructive approach to design the complete contact law with friction and improved numerical algorithm, Math. Comput. Modelling, 28 (1998), pp. 225-245.

[220] K. Deimling, Multivalued Differential Equations, Nonlinear Analysis and Applications, De Gruyter, 1992.

[221] E. Delassus, Mémoire sur la théorie des liaisons finies unilatérales, Annales Scientifiques de l' Ecole Normale Supèrieure, Paris, 34 (1917), pp. 95-179.

[222] Z. Deng, X. NiAn, And C. Hu, Distributed algorithm design for nonsmooth resource allocation problems, IEEE Transactions on Cybernetics, (2019). DoI: 10.1109/TCYB.2019.2901256.

[223] N. Dhingra, S. Khong, And M. Jovanovic, The proximal augmented Lagrangian method for nonsmooth composite optimization, IEEE Transactions on Automatic Control, 64 (2019), pp. 2861-2868.

[224] M. Di Bernardo, C.-J. Budd, A.-R. Champneys, and P. Kowalczyk, Piecewise-smooth Dynamical Systems. Theory and Applications, vol. 163 of Applied Mathematical Sciences, Springer Verlag, London, 2008.

[225] S. di Marino, B. Maury, and F. Santambrogio, Measure sweeping processes, Journal of Convex Analysis, 23 (2016), pp. 1923-2014.

[226] L. Dieci ANd L. Lopez, Sliding motion on discontinuity surfaces of high co-dimension. A construction for selecting a Filippov vector field, Numerische Mathematik, 117 (2011), pp. $779-811$.

[227] M.-V. Dolgopolik And A.-L. Fradkov, Nonsmooth and discontinuous speed-gradient algorithms, Nonlinear Analysis: Hybrid Systems, 25 (2017), pp. 99-113.

[228] J. Dong, D. Zhang, And A. Nagurney, A projected dynamical systems model of general financial equilibrium with stability analysis, Mathematical and Computer Modelling, 24 (1996), pp. 35-44.

[229] X. Dong AND Y. GAO, The equivalence between a complementarity control system and a differential inclusion, International Journal of Nonlinear Science, 5 (2008), pp. 106-110.

[230] A. Doris, A.-L. Juloski, N. Mihajlovic, W.-P.-M.-H. Heemels, N. van De Wouw, And H. NiJMeIJER, Observer designs for experimental non-smooth and discontinuous systems, IEEE Transactions on Control Systems Technology, 16 (2008), pp. 1323-1332.

[231] B. Du AND D.-Z.-W. WANG, Continuum modelling of park-and-ride services considering travel time reliability and heterogeneous commuters-a linear complementarity system approach, Transportation Research Part E, 71 (2014), pp. 58-81.

[232] P. Dupuis And A. Nagurney, Dynamical systems and variational inequalities, Annals of Operations Research, 44 (1993), pp. 9-42.

[233] R. Dzonou And M.-D.-P. Monteiro-Marques, A sweeping process approach to inelastic contact problems with general inertia operators, European Journal of Mechanics A/Solids, 26 (2007), pp. 474-490.

[234] R. Dzonou, M.-D.-P. Monteiro-Marques, and L. Paoli, A convergence result for a vibroimpact problem with a general inertia operator, Nonlinear Dynamics, 58 (2009), pp. 361384.

[235] J.-F. Edmond, Delay perturbed sweeping process, Set-Valued Analysis, 14 (2006), pp. 295-317.

[236] J.-F. Edmond, Relaxation of a Bolza problem governed by a time-delay sweeping process, Set-Valued Analysis, 16 (2008), pp. 563-580.

[237] J.-F. Edmond And L. Thibault, Relaxation of an optimal control problem involving a perturbed sweeping process, Mathematical Programming A, 104 (2005), pp. 347-373.

[238] J.-F. Edmond and L. Thibault, BV solutions of nonconvex sweeping process differential inclusion with perturbation, Journal of Differential Equations, 226 (2006), pp. 135-179.

[239] G. Eichfelder and G. Jahn, Set-semidefinite optimization, Journal of Convex Analysis, 15 (2008), pp. 767-801.

[240] F. FACChinei And J.-S. PANG, Finite-Dimensional Variational Inequalities and Complemen- 
tarity Problems. Volume I, Springer Series in Operations Research, Springer Verlag, New York, 2003.

[241] L. A. FAIK AND A. Syam, Differential inclusions governed by a nonconvex sweeping process, Journal of Nonlinear and Convex Analysis, 2 (2001), pp. 381-392.

[242] M. Falcone and P. Saint-Pierre, Slow and quasi-slow solutions of differential inclusions, Nonlinear Analysis: Theory, Methods and Applications, 11 (1987), pp. 367-377.

[243] H. Fatoorehchi, H. Abolghasemi, and R. Zarghami, Analytical approximate solutions for a general nonlinear resistor-nonlinear capacitor circuit model, Applied Mathematical Modelling, 39 (2015), pp. 6021-6031.

[244] D. Feijer AND F. PAGANini, Stability of primal-dual gradient dynamics and applications to network optimization, Automatica, 46 (2010), pp. 1974-1981.

[245] G. Feng And X. TAN, Absolute stability of a class of singular nonlinear systems with setvalued mappings, Journal of Circuits, Systems, and Computers, 24 (2015), pp. 1550015-1.

[246] A. Ferrara and V. UtKin, Sliding mode optimization in dynamic LTI systems, Journal of Optimization Theory and Applications, 115 (2002), pp. 727-740.

[247] L. Ferreira, E. Kaszkurewicz, And A. Bhaya, Solving systems of linear equations via gradient systems with discontinuous righthand sides: application to LS-SVM, IEEE Transactions on Neural Networks, 16 (2005), pp. 501-505.

[248] A.-F. FILIPpov, Differential equations with discontinuous right-hand side, Matematicheskii Sbornik, 51 (1960), pp. 99-128.

[249] A.-F. Filippov, Differential Equations with Discontinuous Righthand Sides, Mathematics and Its Applications, Kluwer Academic Publishers, Dordrecht, 1988.

[250] N. Fischer, R. Kamalapurkar, and W.-E. Dixon, LaSalle-Yoshizawa corollaries for nonsmooth systems, IEEE Transactions on Automatic Control, 58 (2013), pp. 2333-2338.

[251] S.-D. Flam And A. Ben-Israel, A continuous approach to oligopolistic market equilibrium, Operations Research, 38 (1990), pp. 1045-1051.

[252] F. Forni, A. Tell, And L. ZaCCARIAn, Follow the bouncing ball: Global results on tracking and state estimation with impacts, IEEE Transactions on Automatic Control, 58 (2013), pp. $1470-1485$.

[253] M. Forti, M. Grazzini, P. Nistri, and L. Pancioni, Generalized Lyapunov approach for convergence of neural networks with discontinuous or non-Lipschitz activations, Physica D: Nonlinear Phenomena, 214 (2006), pp. 88-99.

[254] M. Forti, P. Nistri, AND M. QuincamPoix, Generalized neural network for nonsmooth nonlinear programming problems, IEEE Transactions on Circuits and Systems I: Regular Papers, 51 (2004), pp. 1741-1754.

[255] M. ForTi, P. NistRI, AND M. QuincAmPoix, Convergence of neural networks for programming problems via a nonsmooth Lojasiewicz inequality, IEEE Transactions on Neural Networks, 17 (2006), pp. 1471-1486.

[256] R. Frasca, M.-K. Camlibel, I.-C. Goknar, L. IAnnelli, and F. VAsca, Linear passive networks with ideal switches: Consistent initial conditions and state discontinuities, IEEE Transactions on Circuits and Systems, 57 (2010), pp. 3138-3151.

[257] E. Fridman, L. Fridman, And E. Shustin, Steady modes in relay control systems with time delay and periodic disturbances, ASME Journal of Dynamic Systems, Measurement and Control, 122 (2000), pp. 732-737.

[258] T.-L. Friesz, D. Bernstein, N.-J. Mehta, and R.-L. T. S. Ganjalizadeh, Day-to-day dynamic network disequilibria and idealized traveler information systems, Operations Research, 42 (1994), pp. 1120-1136.

[259] T.-L. Friesz, M.-A. Rigdon, And R. MookherJee, Differential variational inequalities and shipper dynamic oligopolistic network competition, Transportation Research Part B: Methodological, 40 (2006), pp. 480-503.

[260] S.-A. Gabriel, S.-A. Kydes, and P. Whitman, The national energy modeling system: A large-scale energy-economic equilibrium model, Operations Research, 49 (2001), pp. 1425.

[261] D. Gadjov and L. Pavel, A passivity-based approach to Nash equilibrium seeking over networks, IEEE Transactions on Automatic Control, 64 (2018), pp. 1077-1092.

[262] S. Galeani, L. Menini, And A. Tornambé, A high gain observer for the estimation of velocity and coefficient of restitution in non-smooth mechanical systems, International Journal of Modelling, Identification and Control, 4 (2008), pp. 44-58.

[263] K. Garg, M. Baranwal, R. Gupta, R. Vasudevan, and D. Panagou, Fixed-time stable proximal dynamical system for solving mixed variational inequality problems. arXiv:1908.03517v2, August 2019.

[264] A.-K. Gelig And G.-A. LeOnov, Monostability of multicoupled systems with discontinuous 
monotonic nonlinearities and non-unique equilibrium position, Automation and Remote Control, 6 (1973), pp. 158-161.

[265] F. GÉnot and B. Brogliato, New results on Painlevé paradoxes, European Journal of Mechanics A/Solids, 18 (1999), pp. 653-678.

[266] C. Georgescu, B. Brogliato, and V. Acary, Switching, relay and complementarity systems: a tutorial on their well-posedness and relationships, Physica D: Nonlinear Phenomena, 241 (2012), pp. 1985-2002.

[267] H. GFrerer and J.-V. OUtrata, On computation of generalized derivatives of the normalcone mapping and their applications, Mathematics of Operations Research, 41 (2016), pp. 1161-1207.

[268] B. Gharesifard and J. Cortès, Distributed convergence to Nash equilibria in two-network zero-sum games, Automatica, 49 (2013), pp. 1683-1692.

[269] P. Gidoni, Rate-independent soft crawlers, The Quarterly Journal of Mechanics and Applied Mathematics, 71 (2018), pp. 369-409.

[270] M.-P. Glazos, S. HuI, AND S.-H. ZaK, Sliding modes in solving convex programming problems, SIAM Journal on Control and Optimization, 36 (1998), pp. 680-697.

[271] C. Glocker, Impacts with global dissipation index at reentrant corners, in Proc. 3rd Contact Mechanics International Symposium. Solid Mechanics and its Applications, M. J.-A.-C. and M.-M. M.-D-P., eds., vol. 103, Dordrecht, 2001, Kluwer Academic, pp. 45-52.

[272] C. Glocker, Set-Valued Force Laws. Dynamics of Non-Smooth Systems, vol. 1 of Lecture Notes in Applied Mechanics, Springer-Verlag, 2001.

[273] C. Glocker, An introduction to impacts, in Nonsmooth Mechanics of Solids, J. Haslinger and G. Stavroulakis, eds., vol. 485 of CISM Courses and Lectures, Wien, 2006, Springer Verlag.

[274] R. Goebel, Lyapunov functions and duality for convex processes, SIAM journal on Control and Optimization, 51 (2013), pp. 3332-3350.

[275] R. Goebel, Stability and robustness for saddle-point dynamics through monotone mappings, Systems and Control Letters, 108 (2017), pp. 16-22.

[276] R. Goebel And R.-G. SANFelice, A unifying convex analysis and switching system approach to consensus with undirected communication graphs, Automatica, 111 (2020). Paper 108598.

[277] D. Goeleven, Existence and uniqueness for a linear mixed variational inequality arising in electrical circuits with transistors, Journal of Optimization Theory and Applications, 138 (2008), pp. 397-406.

[278] D. Goeleven, Complementarity and Variational Inequalities in Electronics, Mathematical Analysis and its Applications, Academic Press, 2017.

[279] D. Goeleven, On the spider that spits the solution of a nonsmooth oscillator, Mathematical Biosciences, 283 (2017), pp. 7-12.

[280] D. Goeleven AND B. BRogliato, Stability and instability matrices for linear evolution variational inequalities, IEEE Transactions on Automatic Control, 49 (2004), pp. 521-534.

[281] D. Goeleven and B. Brogliato, Necessary conditions of asymptotic stability for unilateral dynamical systems, Nonlinear Analysis: Theory, Methods and Applications, 61 (2005), pp. 961-1004.

[282] D. Goeleven, D. Motreanu, Y. Dumont, And M. Rochdi, Variational and Hemivariational Inequalities, Theory, Methods and Applications, I: Unilateral Analysis and Unilateral Mechanics, vol. 69 of Nonconvex Optimization and Its Applications, Kluwer Academic Press, Dordrecht, NL, 2003.

[283] D. Goeleven, D. Motreanu, and V. Motreanu, On the stability of stationary solutions of first-order evolution variational inequalities, Advances in Nonlinear Variational Inequalities, 6 (2003), pp. 1-30.

[284] S. Greenhalgh, V. Acary, and B. Brogliato, Preservation of the dissipativity properties of a class of nonsmooth dynamical systems with the $(\theta, \gamma)$-algorithm, Numerische Mathematik, 125 (2013), pp. 601-637.

[285] S. Greenhalgh and M.-G. Cojocaru, Operations Research, Engineering, and Cyber Security, vol. 113 of Springer Optimization and Its Applications, Springer International Publishing, 2017, ch. Non-equilibrium solutions of dynamic networks: a hybrid system approach, pp. 299-315.

[286] O. GüLER, On the convergence of the proximal point algorithm for convex minimization, SIAM Journal on Control and Optimization, 29 (1991), p. 403-419.

[287] L. Guo AND J. Ye, Necessary optimality conditions for optimal control problems with equilibrium constraints, SIAM Journal on Control and Optimization, 54 (2016), pp. 2710-2733.

[288] L. Gurvits, R. Shorten, And O. Mason, On the stability of switched positive linear systems, 
IEEE Transactions on Automatic Control, 52 (2007), pp. 1099-1103.

[289] J. Gwinner, On differential variational inequalities and projected dynamical systemsequivalence and a stability result, Discrete and Continuous Dynamical Systems, Supplement, (2007), pp. 467-476.

[290] J. Gwinner, On a new class of differential variational inequalities and a stability result, Mathematical Programming B, 139 (2013), pp. 205-221.

[291] N.-T.-T. Ha, J.-J. Strodiot, And P.-T. VuOng, On the global exponential stability of a projected dynamical system for strongly pseudomonotone variational inequalities, Optimization Letters, 12 (2018), pp. 1625-1638.

[292] T.-X.-D. HA AND M.-D.-P. MonTEIRO-Marques, Nonconvex second-order differential inclusions with memory, Set-Valued Analysis, 3 (1995), pp. 71-86.

[293] T. HADDAD, Nonconvex differential variational inequality and state-dependent sweeping process, Journal of Optimization Theory and Applications, 159 (2013), pp. 386-398.

[294] T. Haddad and T. Haddad, Delay perturbed state-dependent sweeping process, Applicable Analysis, 95 (2016), pp. 270-282.

[295] T. Haddad, A. Jourani, ANd L. Thibault, Reduction of sweeping process to unconstrained differential inclusion, Pacific Journal of Optimization, 4 (2008), pp. 493-512.

[296] T. Haddad, J. Noel, And L. Thibault, Perturbed sweeping process with a submooth set depending on the state, Linear and Nonlinear Analysis, 2 (2016), pp. 155-174.

[297] L. Han, M.-K. Camlibel, J.-S. Pang, and W.-P.-M.-H. Heemels, A unified numerical scheme for linear-quadratic optimal control problems with joint control and state constraints, Optimization Methods and Software, 27 (2012), pp. 761-799.

[298] L. HAN AND J.-S. PANG, Non-Zenoness of a class of differential quasi-variational inequalities, Mathematical Programming A, 121 (2010), pp. 171-199.

[299] L. Han, A. Tiwari, M.-K. Camlibel, and J. Pang, Convergence of time-stepping schemes for passive and extended linear complementarity systems, SIAM Journal on Numerical Analysis, 47 (2009), pp. 3768-3796.

[300] A. Hantoute ANd M. Mazade, Lyapunov functions for evolution variational inequalities with uniformly prox-regular sets, Positivity, 21 (2017), pp. 423-448.

[301] A. Hantoute And E. Vilches, Lyapunov pairs for perturbed sweeping processes, Optimization Letters, (2018). https://doi.org/10.1007/s11590-018-1231-4.

[302] T. Hatanaka, N. Chopra, T. Ishizaki, and N. Li, passivity-based distributed optimization with communication delays using PI consensus algorithm, IEEE Transactions on Automatic Control, 63 (2018), pp. 4421-4428.

[303] A. Hauswirth, S. Bolognani, And F. Dorfler, Projected dynamical systems on irregular, non-euclidean domains for nonlinear optimization. https://arxiv.org/abs/1809.04831, 2018.

[304] W.-P.-M.-H. Heemels And B. Brogliato, The complementarity class of hybrid dynamical systems, European Journal of Control, 9 (2003), pp. 322-360.

[305] W.-P.-M.-H. Heemels, M.-K. Camlibel, and J.-M. Schumacher, A time-stepping method for relay systems, in Proc. IEEE 39th Conference on Decision and Control, Sydney, Australia, December 2000, pp. 4461-4466.

[306] W.-P.-M.-H. Heemels, M.-K. Camlibel, J.-M. Schumacher, and B. Brogliato, Observerbased control of linear complementarity systems, Int. J. of Robust and Nonlinear Control, 21 (2011), pp. 1193-1218.

[307] W.-P.-M.-H. Heemels, J.-M. Schumacher, and S. Weiland, Linear complementarity systems, SIAM Journal on Applied Mathematics, 60 (2000), pp. 1234-1269.

[308] W.-P.-M.-H. Heemels, J.-M. Schumacher, and S. Weiland, Projected dynamical systems in a complementarity formalism, Oper. Res. Lett., 27 (2000), pp. 83-91.

[309] A. Hempel, Control of Piecewise Affine Systems Through Inverse Optimization, PhD thesis, ETH Zurich, 2016.

[310] C. Henry, Differential equations with discontinuous right-hand side for planning procedures, Journal of Economic Theory, 4 (1972), pp. 545-551.

[311] C. Henry, An existence theorem for a class of differential equations with multivalued righthand side, Journal of Mathematical Analysis and Applications, 41 (1973), pp. 179-186.

[312] D. Henry, Geometric Theory of Semilinear Parabolic Equations, vol. 840 of Lecture Notes in Mathematics, Springer-Verlag, Berlin Heidelberg New-York, 1981.

[313] J.-B. Hiriart-Urruty and C. Lemaréchal, Convex Analysis and Minimization Algorithms, vol. I and II, Springer Verlag, Heidelberg, 1993.

[314] J.-B. Hiriart-Urruty and C. Lemaréchal, Fundamentals of Convex Analysis, Grundlehren Text Editions, Springer Verlag, 2001.

[315] J.-B. Hiriart-Urruty And A. SEeger, A variational approach to copositive matrices, SiAM 
Review, 52 (2010), pp. 593-629.

[316] N.-D. Hoang AND B.-S. Mordukovich, Extended Euler-Lagrange and Hamiltonian conditions in optimal control of sweeping processes with controlled moving sets, Journal of Optimization Theory and Applications, 180 (2019), pp. 256-289.

[317] S. Hu, Differential equations with discontinuous right-hand sides, Journal of Mathematical Analysis and Applications, 154 (1991), pp. 377-390.

[318] J. Huang, Y. Yang, Z. Han, And J. Zhang, Robust absolute stability criterion for uncertain Lur'e differential inclusion systems with time delay, Circuits, Systems, and Signal Processing, 31 (2012), pp. 2001-2017.

[319] J. Huang, W. Zhang, M. Shi, L. Chen, and L. Yu, Ho observer design for singular onesided lur'e differential inclusion system, Journal of the Franklin Institute, 354 (2017), pp. 3305-3321.

[320] O. Huber, V. ACARY, And B. Brogliato, Lyapunov stability and performance analysis of the implicit discrete sliding mode control, IEEE Transactions on Automatic Control, 61 (2016), pp. 3016-3030.

[321] L. IANnelli, R. Iervolino, And F. VASCA, Linear complementarity systems and conecopositive Lyapunov stability, IEEE Control Systems Letters, 3 (2019), pp. 799-804.

[322] L. IAnnelli, F. VASCA, AND G. Angelone, Computation of steady-state oscillations in power converters through complementarity, IEEE Transactions on Circuits and Systems-I: Regular Papers, 58 (2011), pp. 1421-1432.

[323] R. IERvolino, D. TANGREd, AND F. VASCA, Lyapunov stability for piecewise affine systems via cone-copositivity, Automatica, 81 (2017), pp. 22-29.

[324] R. Iervolino, F. VASCA, And L. IAnNelli, Cone-copositive piecewise quadratic Lyapunov functions for conewise linear systems, IEEE Transactions on Automatic Control, 60 (2015), pp. 3077-3082.

[325] J. ImURA, Well-posedness analysis of switch-driven piecewise affine systems, IEEE Transactions on Automatic Control, 48 (2003), pp. 1926-1935.

[326] J. ImURA AND A.-J. VAN DER SCHAFT, Characterization of well-posedness of piecewise-linear systems, IEEE Transactions on Automatic Control, 45 (2002), pp. 1600-1619.

[327] G. ISAC AND M.-G. CojocARU, The projection operator in a Hilbert space and its directional derivative. Consequences for the theory of projected dynamical systems, Journal of Functions Spaces and Applications, 2 (2004), pp. 71-95.

[328] T. Iто, A Filippov solution of a system of differential equations with discontinuous right-hand sides, Economics Letters, 4 (1979), pp. 359-364.

[329] A.-P. Ivanov, Analytical methods in the theory of vibro-impact systems, J. of Applied Mathematics and Mechanics, 57 (1993), pp. 221-236.

[330] A.-P. Ivanov, The stability of equilibrium in systems with friction, Journal of Applied Mathematics and Mechanics, 71 (2007), pp. 385-395.

[331] A.-P. Ivanov, The equilibrium of systems with dry friction, Journal of Applied Mathematics and Mechanics, (2015). Appeared in Prikl. Mat. Mekh., vol.79, no 3, pp.317-333, 2015.

[332] A.-P. Ivanov And A.-P. MarkeEv, The dynamics of systems with unilateral constraints, J. of Applied Mathematics and Mechanics, 48 (1984), pp. 448-451.

[333] B. Jayawardhana, H. Logemann, and E.-P. Ryan, Input-to-state stability of differential inclusions with applications to hysteretic and quantized feedback systems, SIAM Journal on Control and Optimization, 48 (2009), pp. 1031-1054.

[334] B. Jayawardhana, H. Logemann, and E.-P. Ryan, The circle criterion and input-to-state stability: New perspectives on a classical result, IEEE Control Systems Magazine, 31 (2011), pp. 32-67.

[335] B. Jayawardhana, R. Ouyang, and V. Andrieu, Stability of systems with the Duhem hysteresis operator: the dissipativity approach, Automatica, 48 (2012), pp. 2657-2662.

[336] Z.-P. JiANG AND T. LIU, Small-gain theory for stability and control of dynamical networks: a survey, Annual Reviews in Control, 46 (2018), pp. 58-79.

[337] A. JOKIC, M. LAZAR, AND P.-J. VAN DEN BOSCH, On constrained steady-state regulation: dynamic KKT controllers, IEEE Transactions on Automatic Control, 54 (2009), pp. 22502254 .

[338] A. Jourani And E. Vilches, Positively $\alpha$-far sets and existence results for generalized perturbed sweeping processes, Journal of Convex Analysis, 23 (2016), pp. 775-821.

[339] A. Jourani And E. Vilches, Galerkin-like method and generalized perturbed sweeping process with nonregular sets, SIAM Journal on Control and Optimization, 55 (2017), pp. 24122436.

[340] A. Jourani And E. Vilches, Moreau-Yosida regularization of state-dependent sweeping processes with nonregular sets, Journal of Optimization Theory and Applications, 173 (2017), 
pp. 91-116.

[341] A. Jourani and E. Vilches, A differential equation approach to implicit sweeping processes, Journal of Differential Equations, 266 (2019), pp. 5168-5184.

[342] R.-S. Kamath, L.-T. Biegler, and I.-E. Grossmann, An equation-oriented approach for handling thermodynamics based on cubic equation of state in process optimization, Computers and Chemical Engineering, 34 (2010), pp. 2085-2096.

[343] M. Kamenskil and O. MaKarenkov, On the response of autonomous sweeping processes to periodic perturbations, Set-Valued and Variational Analysis, 24 (2016), pp. 551-563.

[344] M. Kamenskit, O. Makarenkov, L.-N. Wadippuli, and P. R. De Fittec, Global stability of almost periodic solutions to monotone sweeping processes and their response to nonmonotone perturbations, Nonlinear Analysis Hybrid Systems, 30 (2018), pp. 213-224.

[345] C. KANZOW AND D. STECK, On error bounds and multiplier methods for variational problems in Banach spaces, SIAM Journal on Control and Optimization, 56 (2018), pp. 1716-1738.

[346] T. Kato, Nonlinear semigroups and evolution equations, J. Math. Soc. Japan, 19 (1967), pp. $508-520$.

[347] T.-D. Ke, N.-V. LOI, And V. OBukhovskil, Decay solutions for a class of fractional differential variational inequalities, Fractional Calculus and Applied Analysis, 16 (2015), pp. 531-553.

[348] N. Kenmochi, Solvability of nonlinear evolution equations with time-dependent constraints and applications, Bulletin of the Faculty of Education, Chiba University, 30 (1981), pp. 187.

[349] H.-K. Khalil, Nonlinear Systems, Prentice Hall, 3rd ed., 2002.

[350] K.-A. Khan AND P.-I. BARTON, A vector forward mode of automatic differentiation for generalized derivative evaluation, Optimization Methods and Software, 30 (2015), pp. 11851212 .

[351] R. Kikuuwe and B. Brogliato, A new representation of systems with frictional unilateral constraints and its Baumgarte-like relaxation, Multibody System Dynamics, 39 (2017), pp. 267-290.

[352] E. KIM, J. YANG, H. Hwang, AND C.-W. Shul, Impact and blast mitigation using locally bresonant woodpile metamaterials, International Journal of Impact Engineering, 101 (2017), pp. 24-31.

[353] O. Klein And V. Recupero, Hausdorff metric BV discontinuity of sweeping processes, Journal of Physics: conference Series, 727 (2016), p. 012006.

[354] J. Kopfova And V. ReCupero, BV-norm continuity of sweeping processes driven by a set with constant shape, Journal of Differential Equations, 261 (2016), pp. 5875-5899.

[355] S.-K. Korovin And V.-I. UtKin, Use of the slip mode in problems of static optimization, Automation and Remote Control, 33 (1972), pp. 570-579.

[356] S.-K. Korovin AND V.-I. UTKIn, Sliding mode based solution of static optimization and mathematical programming problems. applied aspects, in Preprints of IFAC-IFORS Symposium, Varnia, Bulgaria, 1974, pp. 1-8.

[357] S.-K. Korovin AND V.-I. UTKIN, Using sliding modes in static optimization and nonlinear programming problems, Automatica, 10 (1974), pp. 525-532.

[358] S.-K. Korovin AND V.-I. UtKin, Method of piecewise-smooth penalty functions, Automation and Remote Control, 37 (1976), pp. 39-48.

[359] A.-M. Krasnosel'Skit And A.-V. Pokrovskil, Dissipativity of a resonant pendulum with ferromagnetic friction, Automation and Remote Control, 67 (2002), pp. 221-232.

[360] P. KREJCI, Evolution variational inequalities and multidimensional hysteresis operators, in Nonlinear Differential Equations, vol. 404 of Research Notes in Mathematics, Chapman \& Hall CRC, 1999, pp. 47-111.

[361] P. Krejci And V. Recupero, BV solutions of rate independent differential inclusions, Mathematica Bohemica, 139 (2014), pp. 607-619.

[362] P. Krejci And V. Recupero, Comparing BV solutions of rate independent processes, Journal of Convex Analysis, 21 (2014), pp. 121-146.

[363] P. KReJci And T. Roche, Lipschitz continuous data dependence of sweeping processes in BV spaces, Discrete and Continuous Dynamical Systems series B, 15 (2011), pp. 637-350.

[364] P. Krejci And A. Vladimirov, Lipschitz continuity of polyhedral Skorokhod maps, Journal for Analysis and its Applications (Zeitschrift für Analysis und ihre Anwendungen), 20 (2001), pp. 817-844.

[365] P. KRejci and A. Vladimirov, Polyhedral sweeping processes with oblique reflection in the space of regulated functions, Set-Valued Analysis, 11 (2003), pp. 91-110.

[366] K. Kuhnen and P. KrejcI, Compensation of complex hysteresis and creep effects in piezoelectrically actuated systems-a new Preisach modeling approach, IEEE Transactions on 
Automatic Control, 54 (2009), pp. 537-550.

[367] M. Kunze And M.-D.-P. Monteiro-Marques, Yosida-Moreau regularization of sweeping processes with unbounded variation, Journal of Differential Equations, 130 (1996), pp. 292-306.

[368] M. KunZe and M.-D.-P. Monteiro-Marques, BV solutions to evolution problems with timedependent domains, Set-Valued Analysis, 5 (1997), pp. 57-72.

[369] M. KunZe And M.-D.-P. Monteiro-Marques, Existence of solutions for degenerate sweeping processes, Journal of Convex Analysis, 4 (1997), pp. 165-176.

[370] M. Kunze And M.-D.-P. Monteiro-Marques, On parabolic quasi-variational inequalities and state-dependent sweeping processes, Topol. Methods Nonlinear Anal., Journal of the Juliusz Schauder Center, 12 (1998), pp. 179-191.

[371] M. Kunze and M.-D.-P. Monteiro-Marques, On the discretization of degenerate sweeping processes, Portugaliae Mathematica, 55 (1998), pp. 219-232.

[372] M. Kunze And M.-D.-P. Monteiro-Marques, An introduction to Moreau's sweeping process, in Impacts in Mechanical Systems. Analysis and Modelling, B. Brogliato, ed., vol. 551 of Lecture Notes in Physics, Berlin Heidelberg, 2000, Springer-Verlag, pp. 1-60.

[373] C. Landry, A. Caboussat, and E. Hairer., Solving optimization-constrained differential equations with discontinuity points, with application to atmospheric chemistry, SIAM Journal on Scientific Computing, 31 (2009), pp. 3806-3826.

[374] B.-K. LE, On properties of differential inclusions with prox-regular sets, Pacific Journal of Optimization, 13 (2017), pp. 17-27.

[375] B.-K. LE, Existence of solutions for sweeping processes with local conditions, Journal of Convex Analysis, 27 (2020).

[376] B.-K. LE, Well-posedness and nonsmooth Lyapunov pairs for state-dependent maximal monotone differential inclusions, Optimization, (2020). DoI: 10.1080/02331934.2019.1686504.

[377] D.-M.-W. Leenaerts, On linear dynamic complementary systems, IEEE Transactions on Circuits and Systems I: Fundamental Theory and Applications, 46 (1999), pp. 1022-1026.

[378] D.-M.-W. Leenaerts And W.-M.-G. van Bokhoven, Piecewise Linear Modeling and Analysis, Springer Verlag, 1998.

[379] D.-B. LeEson AND S. WeinReB, Frequency multiplication with nonlinear capacitors - A circuit analysis, Proceedings of the IRE, 47 (1959), pp. 2076-2084.

[380] R.-I. LEINE, Non-smooth stability analysis of the parametrically excited impact oscillator, International Journal of Non-Linear Mechanics, 47 (2012), pp. 1020-1032.

[381] R.-I. Leine, U. Aeberhard, and C. Glocker, Hamilton's principle as variational inequality for mechanical systems with impact, Journal of Nonlinear Science, 19 (2009), pp. 633-664.

[382] R.-I. Leine and C. Glocker, A set-valued force law for spatial Coulomb-Contensou friction, European Journal of Mechanics, 22 (2003), pp. 193-216.

[383] R.-I. Leine AND T.-F. HeImSCH, Global uniform symptotic attractive stability of the nonautonomous bouncing ball system, Physica D, 241 (2012), pp. 2029-2041.

[384] R.-I. Leine and H. Nijmeijer, Dynamics and Bifurcations of Non-Smooth Mechanical Systems, vol. 18 of Lecture Notes in Applied and Computational Mechanics, Springer Verlag, Berlin Heidelberg, 2004.

[385] R.-I. Leine AND N. VAN DE Wouw, Stability and Convergence of Mechanical Systems with Unilateral Constraints, vol. 36 of Lecture Notes in Applied and Computational Mechanics, Springer-Verlag, Berlin Heidelberg, 2008.

[386] R.-I. LEINE AND N. VAN DE WOUW, Uniform convergence of monotone measure differential inclusions: with application to the control of mechanical systems with unilateral constraints, International Journal of Bifurcation and Chaos, 15 (2008), pp. 1435-1457.

[387] G.-A. LEONOv, Stability of nonlinear controllable systems having a nonunique equilibrium position, Automation and Remote Control, 10 (1971), pp. 23-28.

[388] W. Li, X. WANG, AND N.-J. HuAng, A system of differential set-valued variational inequalities in finite dimensional spaces, Journal of Function Spaces, (2014). Article ID 918796, 8 pages, doi:10.1155/2014/918796.

[389] X. Li, N.-J. Huang, And D. O'Regan, Differential mixed variational inequalities in finite dimensional spaces, Nonlinear Analysis: Theory, Methods and Applications, 72 (2010), pp. $3875-3886$.

[390] X. Li AND Z. LiU, Sensitivity analysis of optimal control problems described by differential hemivariational inequalities, SIAM Journal on Control and Optimization, 56 (2018), pp. 3569-3597.

[391] D. Liberzon and S. Trenn, Switched nonlinear differential algebraic equations: Solution theory, Lyapunov functions, and stability, Automatica, 58 (2012), pp. 954-963.

[392] M.-R. Liberzon, Essays on the absolute stability theory, Automation and Remote Control, 
67 (2006), pp. 1610-1644.

[393] T. LiU, Z.-P. JiAng, AND D.-J. Hill, A sector bound approach to feedback control of nonlinear systems with state quantization, Automatica, 48 (2012), pp. 145-152.

[394] Z. Liu, D. Motreanu, And S. Zeng, Nonlinear evolutionary systems driven by mixed variational inequalities and its applications, Nonlinear Analysis: Real World Applications, 42 (2018), pp. 409-421.

[395] Z. LiU, S. ZENG, AND D. Motreanu, Evolutionary problems driven by variational inequalities, Journal of Differential Equations, 260 (2016), pp. 6787-6799.

[396] N.-V. Loi, T.-D. Ke, V. Obukhovskir, And P. ZeCCA, Topological methods for some classes of differential variational inequalities, Journal of Nonlinear and Convex Analysis, 17 (2016), pp. 403-419.

[397] Y.-J. Lootsma, A.-J. van DeR Schaft, and M.-K. CAmlibel, Uniqueness of solutions of relay systems, Automatica, 35 (1999), pp. 467-478.

[398] P. LÖtSTEDT, Mechanical systems of rigid bodies subject to unilateral constraints, SIAM Journal on Applied Mathematics, 42 (1984), pp. 281-296.

[399] S. Lounis, T. HADDAD, AND M. Sene, Non-convex second-order Moreau's sweeping process in Hilbert spaces, Journal of Fixed Point Theory and Applications, 19 (2017), pp. 2895-2908.

[400] K. LU, G. JING, AND L. WANG, A distributed algorithm for solving mixed equilibrium problems, Automatica, 105 (2019), pp. 246-253.

[401] A.-I. Lure And V.-N. Postnikov, On the stability theory of control systems, Sov. Appl. Math., 8 (1944), pp. 246-248.

[402] R. MA, X. BAN, AND J.-S. PANG, Continuous-time dynamic system optimum for singledestination traffic networks with queue spillbacks, Transportation Research Part B, 68 (2014), pp. 98-122.

[403] M. Mabrouk, A unified variational model for the dynamics of perfect unilateral constraints, European Journal of Mechanics A/Solids, 17 (1998), pp. 819-842.

[404] A. Machina And A. Ponosov, Filippov solutions in the analysis of piecewise linear models describing gene regulatory networks, Nonlinear Analysis: Theory, Methods and Applications, 74 (2011), pp. 882-900.

[405] A. Machina And A. Ponosov, Stability of stationary solutions of piecewise affine differential equations describing gene regulatory networks, Nonlinear Analysis: Theory, Methods and Applications, 380 (2011), pp. 736-749.

[406] A. Machina, A. Ponosov, and E.-O. Voit, Automated piecewise power-law modeling of biological systems, Journal of Biotechnology, 149 (2010), pp. 154-165.

[407] P. Maffezzoni, L. Codecasa, And D. D'Amore, Event-driven time-domain simulation of closed-loop switched circuits, IEEE Transactions on Computer-Aided design of Integrated Circuits and Systems, 25 (2006), pp. 2413-2426.

[408] S. Manfredi AND D. ANGeli, Necessary and sufficient conditions for consensus in nonlinear monotone networks with unilateral interactions, Automatica, 77 (2017), pp. 51-60.

[409] J.-M. Manley And H.-E. Rowe, Some general properties of nonlinear elements - Part I. General energy relations, Proceedings of the IRE, 44 (1956), pp. 904-913.

[410] S. Marcellin and L. Thibault, Evolution problems associated with primal lower nice functions, Journal of Convex Analysis, 13 (2006), pp. 385-421.

[411] F. Martinelli, L. Menini, And A. Tornambé, Observability, reconstructibility and observer design for linear mechanical systems unobservable in absence of impacts, ASME J. Dyn. Sys., Meas., Control, 125 (2004), pp. 549-562.

[412] B. MaRTinet, Régularisation d'inéquations variationnelles par approximations successives, Revue Française d'Informatique et Recherche Opérationnelle, 4 (1970), pp. 154-158.

[413] B. Maury and J. Venel, A discrete contact model for crowd motion, ESAIM: Mathematical Modelling and Numerical Analysis (ESAIM: M2AN), 45 (2011), pp. 145-168.

[414] M. Mazade And L. Thibault, Differential variational inequalities with locally prox-regular sets, Journal of Convex Analysis, 19 (2012), pp. 1109-1139.

[415] M. Mazade and L. Thibault, Regularization of differential variational inequalities with locally prox-regular sets, Mathematical Programming B, 139 (2013), pp. 243-269.

[416] P. Mehlitz and G. Wachsmuth, The limiting normal cone to pointwise defined sets in Lebesgue spaces, Set-Valued and Variational Analysis, 26 (2018), pp. 449-467.

[417] L. Menini and A. Tornambé, Asymptotic tracking of periodic trajectories for a simple mechanical system subject to nonsmooth impacts, IEEE Transactions on Automatic Control, 46 (2001), pp. 1122-1126.

[418] L. Menini And A. ToRnambé, State estimation of (otherwise unobservable) linear mechanical systems through the use of non-smooth impacts: the case of two mating gears, Automatica, 38 (2002), pp. 1823-1826. 
[419] L. Menini And A. Tornambé, Velocity observers for non-linear mechanical systems subject to non-smooth impacts, Automatica, 38 (2002), pp. 2169-2175.

[420] S. Migorski AND S. ZENG, A class of generalized evolutionary problems driven by variational inequalities and fractional operators, Set-Valued and Variational Analysis, 27 (2019), pp. 949-970.

[421] F.-A. Miranda-Villatoro, B. Brogliato, and F. Castanos, Multivalued robust tracking control of Lagrange systems: Continuous and discrete-time algorithms, IEEE Transactions on Automatic Control, 62 (2017), pp. 4436-4450.

[422] F.-A. Miranda-Villatoro, B. Brogliato, and F. Castanos, Set-valued sliding mode control of uncertain linear systems: Continuous and discrete-time analysis, SIAM Journal on Control and Optimization, 56 (2018), p. 1756-1793.

[423] F.-A. Miranda-Villatoro and F. CAstanos, Robust output regulation of strongly passive linear systems with multivalued maximally monotone controls, IEEE Transactions on Automatic Control, 62 (2017), pp. 238-249.

[424] F.-A. Miranda-Villatoro, F. Castanos, and B. Brogliato, Continuous and discrete-time stability of a robust set-valued nested controller, Automatica, 107 (2019), pp. 406-417.

[425] M.-D.-P. Monteiro-Marques, Perturbations convexes semi-continues supérieurement de problèmes d'évolution dans les espaces de Hilbert, in Travaux du Séminaire d'Analyse Convexe de Montpellier, Exposé 2, University of Montpellier, France, 1984.

[426] M.-D.-P. Monteiro-Marques, Chocs inélastiques standards: un résultat d'existence, in Séminaire d'Analyse Convexe, exposé 4, preprint 85-3, Université de Montpellier, France, 1985.

[427] M.-D.-P. Monteiro-Marques, Differential Inclusions in Nonsmooth Mechanical Problems. Shocks and Dry Friction, vol. 9, Birkhäuser, Basel, 1993.

[428] C.-I. Morarescu and B. Brogliato, Passivity-based switching control of flexible-joint complementarity mechanical systems, Automatica, 46 (2010), pp. 160-166.

[429] C.-I. Morarescu And B. Brogliato, Trajectory tracking control of multiconstraint complementarity lagrangian systems, IEEE Transactions on Automatic Control, 55 (2010), pp. 1300-1313.

[430] B.-S. Mordukhovich, Variational Analysis and Generalized Differentiation I: Basic Theory, vol. 330 of Comprehensive Studies in Mathematics, Springer Verlag, Berlin, 2006.

[431] B.-S. Mordukhovich, Variational Analysis and Applications, Springer Monographs in Mathematics, Springer International Publishing AG, Cham, CH, 2018.

[432] J.-J. Moreau, Les liaisons unilatérales et le principe de Gauss, C.R. Acad. Sci. Paris, 256 (1963), pp. 871-874.

[433] J.-J. Moreau, Quadratic programming in mechanics: dynamics of one sided constraints, J. SIAM Control series A, 4 (1966), pp. 153-158.

[434] J.-J. Moreau, Rafle par un convexe variable I. Sém. Anal. Convexe Montpellier, France, exposé $15,1971$.

[435] J.-J. Moreau, Rafle par un convexe mobile dans un espace hilbertien, in Colloque d'Analyse Non-linéaire, Pau, France, 1972. https://hal.inria.fr/hal-01795265.

[436] J.-J. Moreau, Rafle par un convexe variable II. Sém. Anal. Convexe Montpellier, France, exposé 3, 1972.

[437] J.-J. Moreau, On unilateral constraints, friction and plasticity, in New Variational Techniques in Mathematical Physics, G. Capriz and G. Stampacchia, eds., CIME II Ciclo, Roma, 1974, Ediz. Cremonese, pp. 173-222. https://hal.inria.fr/hal-01793413.

[438] J.-J. Moreau, Application of convex analysis to the treatment of elastoplastic systems, in Applications of Methods of Functional Analysis to Problems of Mechanics, Joint Symposium IUTAM/IMU, P. Germain and B. Nayroles, eds., Lecture Notes in Mathematics, Marseille, France, 1976, Springer Verlag, pp. 56-89.

[439] J.-J. MoREAU, Evolution problem associated with a moving convex set in a Hilbert space, Journal of Differential Equations, 26 (1977), pp. 347-374. Preliminary version in: Problème d'évolution associé à un convexe mobile d'un espace Hilbertien. Comptes Rendus de l'Académie des Sciences. Série IIb, Mécanique, Elsevier, 1973, 276, pp.791-794.

[440] J.-J. Moreau, Application of convex analysis to some problems of dry friction, in Trends in Applications of Pure Mathematics to Mechanics, H. Zorski, ed., vol. 2, London, 1979, Pitman publishing Ltd, pp. 263-280. https://hal.inria.fr/hal-01864012.

[441] J.-J. Moreau, Liaisons unilatérales sans frottement et chocs inélastiques, C.R. Acad. Sci. Paris, 296 (1983), pp. 1473-1476.

[442] J.-J. Moreau, Unilateral contact and dry friction in finite freedom dynamics, in Nonsmooth Mechanics and Applications, J.-J. Moreau and P.-D. Panagiotopoulos, eds., vol. 302, Wien New York, 1988, CISM Courses and Lectures, Springer Verlag, pp. 1-82. 
[443] J.-J. Moreau, Numerical aspects of the sweeping process, Computer Methods in Applied Mechanics and Engineering, 177 (1999), pp. 329-349.

[444] F. NACRY, Preturbed BV sweeping process involving prox-regular sets, Journal of Nonlinear and Convex Analysis, 18 (2017), pp. 1619-1651.

[445] F. NACRY, Truncated nonconvex state-dependent sweeping process: implicit and semi-implicit adapted Moreau's catching-up algorithms, Journal of Fixed Point Theory and Applications, 20 (2018), p. 121.

[446] F. NACRY AND L. ThiBAult, BV prox-regular sweeping process with bounded truncated variation, Optimization, (2018). https://doi.org/10.1080/02331934.2018.1514039.

[447] A. Nagurney, J. CRuz, AND D. MatsyPura, Dynamics of global supply chain supernetworks, Mathematical and Computer Modelling, 37 (2003), pp. 963-983.

[448] A. NAGURNEY AND J. PAN, Evolution variational inequalities and projected dynamical systems with application to human migration, Mathematical and Computer Modelling, 43 (2006), pp. 646-657.

[449] A. Nagurney and D. Zhang, Projected Dynamical Systems and variational Inequalities, Kluwer, Dordrecht, 1995.

[450] J. NoEL, Second order general perturbed sweeping process differential inclusion, Journal of Fixed Point Theory and Applications, 20 (2018), p. 133.

[451] M.-N. OGuzTöreli, Relay type control systems with retardation and switching delay, J. SIAM Control series A, 1 (1963), pp. 275-289.

[452] Y. Or And A.-D. Ames, Stability and completion of Zeno equilibria in lagrangian hybrid systems, IEEE Transactions on Automatic Control, 56 (2011), pp. 1322-1336.

[453] Y. OR And A. Teel, Zeno stability of the set-valued bouncing ball, IEEE Transactions on Automatic Control, 56 (2011), pp. 447-452.

[454] Y. ORLOV, Finite time stability and robust control synthesis of uncertain switched systems, SIAM Journal on Control and Optimization, 43 (2005), pp. 1253-1271.

[455] Y.-V. Orlov, Discontinuous Systems. Lyapunov Analysis and Robust Synthesis under Uncertainty Conditions, Springer-Verlag, London, UK, 2009.

[456] M. Otani, On existence of strong solutions for $\frac{d u}{d t}(t)+\partial \varphi^{1}(u(t))-\partial \varphi^{2}(u(t))$ э $f(t)$, Journal of the Faculty of Science, the University of Tokyo. Sect. 1 A, Mathematics, 24 (1977), pp. 575-605.

[457] R. Ouyang and B. Jayawardhana, Absolute stability analysis of linear systems with Duhem hysteresis operator, Automatica, 50 (2014), pp. 1860-1866.

[458] H.-B. Oza, Y.-V. Orlov, And S.-K. Spurgeon, Finite time stabilization of a perturbed double integrator with unilateral constraints, Mathematics and Computers in Simulation, 95 (2013), pp. 200-212.

[459] H.-B. Oza, Y.-V. Orlov, And S.-K. Spurgeon, A unified Lyapunov function for finite time stabilization of continuous and variable structure systems with resets, in Proc. IEEE 55th Conference on Decision and Control, Las Vegas USA, 2016, pp. 2201-2206.

[460] J.-S. Pang, L. Han, G. Ramadurai, And S. UkKusuri, A continuous-time linear complementarity system for dynamic user equilibria in single bottleneck traffic flows, Mathematical Programming A, 133 (2012), pp. 437-460.

[461] J.-S. PANG AND J. Shen, Strongly regular differential variational systems, IEEE Transactions on Automatic Control, 52 (2007), pp. 242-255.

[462] J.-S. PAng And D. Stewart, Differential variational inequalities, Mathematical Programming A, 113 (2008), pp. 345-424.

[463] J.-S. PANG AND D. STEWART, Solution dependence on initial conditions in differential variational inequalities, Mathematical Programming B, 116 (2009), pp. 429-460.

[464] L. PAoli, Continuous dependence on data for vibro-impact problems, Math. Models Methods Appl. Sci. (M3AS), 35 (2005), pp. 1-41.

[465] L. PAOLI, Vibro-impact problems with dry friction-part I: Existence result, SIAM Journal on Mathematical Analysis, 47 (2015), pp. 3285-3313.

[466] L. PAOLI, Vibro-impact problems with dry friction - part II: Tangential contacts and frictional catastrophes, SIAM Journal on Mathematical Analysis, 48 (2016), pp. 1272-1296.

[467] L. Paoli and M. Schatzman, Mouvement à un nombre fini de degrés de liberté avec contraintes unilatérales : cas avec perte d'énergie, RAIRO-Modélisation mathématique et analyse numérique, 27 (1993), pp. 673-717.

[468] M. Pappalardo And M. Passacantando, Stability for equilibrium problems: from variational inequalities to dynamical systems, Journal of Optimization Theory and Applications, 113 (2002), pp. 567-582.

[469] T. PARÉ, A. Hassibi, AND J. How, A KYP lemma and invariance principle for systems with multiple hysteresis non-linearities, International Journal of Control, 74 (2001), pp. 1140- 
1157.

[470] N.-H. PAvel, Nonlinear Evolution Operators and Semigroups: Applications to Partial Differential Equations, vol. 1260 of Lecture Notes in Mathematics, Springer, Berlin, 1987.

[471] P. PÉcol, P. Argoul, S. D. Pont, And S. Erlicher, The non-smooth view for contact dynamics by Michel Frémond extended to the modelling of crowd movements, Discrete and Continuous Dynamical Systems Series S, 6 (2013), pp. 547-565.

[472] P. Pécol, S. D. Pont, S. Erlicher, And P. Argoul, Smooth/non-smooth contact modeling of human crowds movement: numerical aspects and application to emergency evacuations, Ann. Solid Struct. Mech., 2 (2011), pp. 69-85.

[473] D. Percivale, Uniqueness in the elastic bounce problem, Journal of Differential Equations, 56 (1985), pp. 206-215.

[474] D. Percivale, Uniqueness in the elastic bounce problem, II, Journal of Differential Equations, 90 (1991), pp. 304-315.

[475] C. D. Persis and S. Grammatico, Continuous-time integral dynamics for monotone aggregative games with coupling constraints, IEEE Transactions on Automatic Control, (2019). DoI: 10.1109/TAC.2019.2939639.

[476] C. D. Persis and S. Grammatico, Distributed averaging integral Nash equilibrium seeking on networks, Automatica, 110 (2019). Article 108548.

[477] J. Peypouquet And S. SoRIn, Evolution equations for maximal monotone operators: asymptotic analysis in continuous and discrete time, Journal of Convex Analysis, 17 (2010), pp. 1113-1163.

[478] F. Pfeiffer and C. Glocker, Multibody Dynamics with Unilateral Contacts, Nonlinear Science, Wiley, 1996.

[479] L.-A. PIPES, Analysis of electric circuits containing noninear resistance, Journal of the Franklin Institute, 263 (1957), pp. 47-55.

[480] A.-Y. Pogromsky, W.-P.-M.-H. Heemels, And H. NiJmeiJer, On solution concepts and well-posedness of linear relay systems, Automatica, 39 (2003), pp. 2139-2147.

[481] A. Polyakov, D. Efimov, and B. Brogliato, Consistent discretization of finite-time and fixed-time stable systems, SIAM Journal on Control and Optimization, 57 (2019), pp. 78 103.

[482] E. Pratt, A. LÉGer, ANd M. Jean, About a stability conjecture concerning unilateral contact with friction, Nonlinear Dynamics, 59 (2010), pp. 73-94.

[483] A.-U. Raghunathan, J.-R. Perez-Correa, E. Agosin, and L.-T. Biegler, Parameter estimation in metabolic flux balance models for batch fermentation-formulation and solution using differential variational inequalities, Ann. Oper. Res., 148 (2006), pp. 251-270.

[484] A.-U. Raghunathan, M. Soledad-Diaz, and L.-T. Biegler, An MPEC formulation for dynamic optimization of distillation operations, Computers and Chemical Engineering, 28 (2009), pp. 2037-2052.

[485] V. Recupero, A continuity method for sweeping process, Journal of Differential Equations, 251 (2011), pp. 2125-2142.

[486] V. Recupero, BV continuous sweeping processes, Journal of Differential Equations, 259 (2015), pp. 4253-4272.

[487] V. RECUPERO, Convex valued geodesics and applications to sweeping processes with bounded retraction, Journal of Convex Analysis, 27 (2020).

[488] V. Recupero And F. SAntambrogio, Sweeping processes with prescribed behaviour on jumps, Annali di Matematica, 197 (2018), pp. 1311-1332.

[489] S. Robinson, Generalized equations and their solutions. I Basic theory, Mathematical Programming Study, 10 (1979), pp. 128-141.

[490] R. Rockafellar, Monotone Processes of Convex and Concave Type, vol. 77 of Memoirs of the American Mathematical Society, American Mathematical Society, 1967. ISBN: 978-1-4704-0025-5.

[491] R.-T. Rockafellar, Convex Analysis, Princeton Landmarks in Mathematics, Princeton University Press, 1970.

[492] R.-T. Rockafellar and R.-J.-B. Wets, Variational Analysis, vol. 317 of Comprehensive Studies in Mathematics, Springer Verlag, Berlin Heidelberg, 1998.

[493] A. Rodriguez-Vasquez, R. Dominguez-Castro, A. Rueda, J.-L. Huertas, and E. SANChEZ-Sinencio, Nonlinear switched-capacitor "neural" networks for optimization problems, IEEE Transactions on Circuits and Systems, 37 (1990), pp. 384-398.

[494] N. Rouche and J. Mawhin, Equations Différentielles Ordinaires, Tome 2, Masson et Cie, Paris, France, 1973.

[495] E.-P. RYAn, An integral invariance principle for differential inclusions with applications in adaptive control, SIAM Journal on Control and Optimization, 36 (1998), pp. 960-980. 
[496] A.-M. Sahlodin, H.-A.-J. Watson, And P.-I. Barton, Nonsmooth model for dynamic simulation of phase changes, AIChE Journal, 62 (2016), pp. 3334-3351.

[497] D. Salas, L. Thibault, and E. Vilches, On smoothness of solutions to projected differential equations, Discrete and Continuous Dynamical Systems, 39 (2019), pp. 2255-2283.

[498] M. Schatzman, A class of nonlinear differential equations of second order in time, Nonlinear Analysis: Theory, Methods and Applications, 2 (1978), pp. 355-373.

[499] M. Schatzman, Uniqueness and continuous dependence on data for one-dimensional impact problems, Mathematical and Computer Modelling, 28 (1998), pp. 1-18. Issue 4-8.

[500] C.-W. Scherer AND T. Holicki, An IQC theorem for relations: towards stability analysis of data-integrated systems, in 9th IFAC Symposium on Robust Control Design, Florianopolis, Brazil, 2018.

[501] M. Schulz And S. Pellegrino, Equilibrium paths of mechanical systems with unilateral constraints I. Theory, Proceedings Royal Soc. London A: Mathematical, Physical and Engineeriong Sciences, 456 (2000), pp. 2223-2242.

[502] J.-M. SCHUMACHeR, Complementarity systems in optimization, Mathematical Programming B, 101 (2004), pp. 263-295.

[503] L. Schwartz, Théorie des Distributions, Publications de l'Institut de Mathématique de l'université de Strasbourg, Hermann, Paris, 1966.

[504] M. Sene and L. Thibault, Regularization of dynamical systems associated with prox-regular moving sets, Journal of Nonlinear and Convex Analysis, 15 (2014), pp. 647-663.

[505] O.-S. SEREA, On reflecting boundary problem for optimal control problem, SIAM Journal on Control and Optimization, 42 (2003), pp. 559-575.

[506] O.-S. SEREA, Optimality conditions for reflecting boundary control problems, Nonlinear Differential Equations and Applications NoDEA, 20 (2013), pp. 1225-1242.

[507] V. Sessa, L. Iannelli, and F. Vasca, A complementarity model for closed-loop power converters, IEEE Transactions on Power Electronics, 29 (2014), pp. 6821-6835.

[508] V. Sessa, L. IAnnelli, F. VAsCa, And V. ACARY, A complementarity approach for the computation of periodic oscillations in piecewise linear systems, Nonlinear Dynamics, 85 (2016), pp. $1255-1273$.

[509] J. Shen And J.-S. PAng, Linear complementarity systems: Zeno states, SIAM Journal on Control and Optimization, 44 (2005), pp. 1040-1066.

[510] J. Shen And J.-S. PAng, Semicopositive linear complementarity systems, Int. Journal of Robust and Nonlinear Control, 17 (2007), pp. 1367-1386.

[511] M.-J. Shi, J. Huang, L. Chen, And L. Yu, Adaptive full-order and reduced-order observers for one-sided Lur'e systems with set-valued mappings, IMA Journal of Mathematical Control and Information, 35 (2016), pp. 569-589.

[512] A.-H. Siddiqi, P. Manchanda, AND M. BRokate, On some recent developments concerning Moreau's sweeping process, in Proceedings of the 1st International Conference on Industrial and Applied Mathematics of the Indian Subcontinent: Trends in Industrial and Applied Mathematics, A. Siddiqi and M. Kocvara, eds., Applied Optimization, Kluwer Academic Publishers, 2002, pp. 339-354.

[513] G.-V. Smirnov, Introduction to the Theory of Differential Inclusions, vol. 41 of Graduate Studies in Mathematics, American Mathematical Society, Provicence, Rhode Island, 2002.

[514] J. Sponsel, S. Bundfuss, And M. Dür, An improved algorithm to test copositivity, Journal of Global Optimization, 52 (2012), pp. 537-551.

[515] J.-S. Spraker And D.-C. Biles, A comparison of the Carathéodory and Filippov solution sets, Journal of Mathematical Analysis and Applications, 198 (1996), pp. 571-580.

[516] P. Stechlinski, M. Patrascu, And P.-I. Barton, Nonsmooth differential-algebraic equations in chemical engineering, Computers and Chemical Engineering, 114 (2018), pp. 52-68.

[517] T. Stegink, T. van Damme, and C. De Persis, Convergence of projected primal-dual dynamics with applications in data centers, in 7th IFAC Workshop on Distributed Estimation and Control in Networked Systems NECSYS, vol. 51 of IFAC PapersOnLine, Groningen, The Netherlands, August 2018, pp. 88-93.

[518] D. Stewart, A high accuracy method for solving ODEs with discontinuity right-hand side, Numerische Mathematik, 58 (1990), pp. 299-328.

[519] D. Stewart, Convergence of a time-stepping scheme for rigid-body dynamics and resolution of Painlevés problem, Archive for Rational Mechanics and Analysis, 145 (1998), pp. 215260.

[520] D.-E. Stewart, Uniqueness for index-one differential variational inequalities, Nonlinear Analysis: Hybrid Systems, 2 (2008), pp. 812-818.

[521] D.-E. Stewart and J.-C. Trinkle, An implicit time-stepping scheme rigid body dynamics with inelastic collisions and Coulomb friction, Int. J. Numer. Methods Eng., 39 (1996), 
pp. 2673-2691.

[522] A. Tanwani, B. Brogliato, and C. Prieur, Stability and observer design for Lur'e systems with multivalued, non-monotone, time-varying nonlinearities and state jumps, SIAM Journal on Control and Optimization, 56 (2014), pp. 3639-3672.

[523] A. Tanwani, B. Brogliato, and C. Prieur, Observer design for unilaterally constrained Lagrangian systems: A passivity-based approach, IEEE Transactions on Automatic Control, 61 (2016), pp. 2386-2401.

[524] A. Tanwani, B. Brogliato, and C. Prieur, Well-posedness and output regulation for implicit time-varying evolution variational inequalities, SIAM Journal on Control and Optimization, 56 (2018), pp. 751-781.

[525] A. Tanwani and S. Trenn, Determinability and state estimation for switched differentialalgebraic equations, Automatica, 76 (2017), pp. 17-31.

[526] A. Tanwani and Q. Zhu, Feedback Nash equilibrium for randomly switching differentialalgebraic games, IEEE Transactions on Automatic Control, (2020). DOI: 10.1109/TAC.2019.2943577.

[527] A. Tasora, M. Anitescu, S. Negrini, and D. Negrut, A compliant visco-plastic particle contact model based on differential variational inequalities, International Journal of NonLinear Mechanics, 53 (2013), pp. 2-12.

[528] A.-A. ten Dam, K.-F. Dwarshuis, and J.-C. Willems, The contact problem for linear continuous-time dynamical systems: a geometric approach, IEEE Transactions on Automatic Control, 42 (1997), pp. 458-472.

[529] L. Thibault, Propriétés des sous-différentiels de fonctions localement lipschitziennes definies sur un espace de Banach séparable. Applications, PhD thesis, Département de Mathématiques, Université de Montpellier, Montpellier, France, 1975.

[530] L. Thibault, Sweeping process with regular and nonregular sets, Journal of Differential Equations, 193 (2003), pp. 1-26.

[531] L. Thibault, Moreau sweeping process with bounded truncated retraction, Journal of Convex Analysis, 23 (2016), pp. 1051-1098.

[532] L.-Q. Thuan And M.-K. Camlibel, Continuous piecewise affine dynamical systems do not exhibit Zeno behavior, IEEE Transactions on Automatic Control, 56 (2011), pp. 19321936.

[533] L.-Q. Thuan and M.-K. Camlibel, Controllability and stabilizability of a class of continuous piecewise affine dynamical systems, SIAM Journal on Control and Optimization, 52 (2014), pp. 1914-1934.

[534] L.-Q. Thuan And M.-K. Camlibel, On the existence, uniqueness and nature of Carathéodory and Filippov solutions for bimodal piecewise affine dynamical systems, Systems and Control Letters, 68 (2014), pp. 76-85.

[535] A.-A. Tolstogonov, Sweeping process with unbounded nonconvex perturbation, Nonlinear Analysis, 108 (2014), pp. 291-301.

[536] A.-A. Tolstogonov, Control sweeping processes, Journal of Convex Analysis, 23 (2016), pp. 1099-1123.

[537] A.-A. Tolstogonov, Relaxation in nonconvex optimal control problems containing the difference of two subdifferentials, SIAM Journal on Control and Optimization, 54 (2016), pp. 175-197.

[538] A.-A. TOlSTOGONov, Existence and relaxation of solutions for a subdifferential inclusion with unbounded perturbation, Journal of Mathematical Analysis and Applications, 447 (2017), pp. 269-288.

[539] A.-A. Tolstogonov, Polyhedral sweeping processes with unbounded nonconvex-valued perturbation, Journal of Differential Equations, 263 (2017), pp. 7965-7983.

[540] A.-A. Tolstogonov, Filippov-Wazewski theorem for subdifferential inclusions with an unbounded perturbation, SIAM Journal on Control and Optimization, 56 (2018), pp. 28782900.

[541] N.-W. Tschoegl, The Phenomenological Theory of Linear Viscoelastic Behaviour. An Introduction, Springer, Berlin, 1989.

[542] V.-I. UtKin, Sliding Modes in Control and Optimization, Communications and Control Engineering, Springer Verlag, Berlin Heidelberg, 1992.

[543] M. VALADIER, Quelques problèmes d'entrainement unilatéral en dimension finie, in Séminaire d'Analyse Convexe, vol. Exposé 8, Montpellier, France, 1988.

[544] M. VALADIER, Lipschitz approximation of the sweeping (or Moreau) process, Journal of Differentila Equations, 88 (1990), pp. 248-264.

[545] M. Valadier, Rafle et viabilité, in Séminaire d'Analyse Convexe, vol. Exposé 17, Montpellier, France, 1992. 
[546] W.-M.-G. van Bokhoven And D.-M.-W. Leenaerts, Explicit formulas for the solutions of piecewise linear networks, IEEE Transactions on Circuits and Systems. I, Fundamental Theory and Applications, 46 (1999), pp. 1110-1117.

[547] N. VAN DE WOuW AND R.-I. LeINE, Attractivity of equilibrium sets of systems with dry friction, Nonlinear Dynamics, 35 (2004), pp. 19-39.

[548] A.-J. VAN DER SCHAFt AND J.-M. SCHUMACHER, The complementary-slackness class of hybrid systems, Mathematics of Control, Signals and Systems, 9 (1996), pp. 266-301.

[549] A.-J. van Der SChaft AND J.-M. Schumacher, Complementarity modeling of hybrid systems, IEEE Transactions on Automatic Control, 43 (1998), pp. 483-490.

[550] N. van Loi, M.-Q. VU, ANd P.-T. CuOng, Nonlocal problem for differential complementarity systems, Applied Mathematics and Computation, 265 (2015), pp. 799-806.

[551] L. Vandenberghe, B.-L. De Moor, and J. VAndewalle, The generalized linear complementarity problem applied to the complete analysis of resistive piecewise-linear circuits, IEEE Transactions on Circuits and Systems, 36 (1989), pp. 1382-1391.

[552] F. Vasca, L. Iannelli, M.-K. Camlibel, and R. Frasca, A new perspective for modeling power electronics converters: complementarity framework, IEEE Transactions on Power Electronics, 24 (2009), pp. 456-468.

[553] M. Vidyasagar, Nonlinear Systems Analysis, Prentice-Hall International Editions, 2nd ed., 1993.

[554] A. Vieira, B. Brogliato, and C. Prieur, Optimality conditions for the minimal time problem for complementarity systems, in Proceedings of 11th IFAC Nonlinear Control Systems NOLCOS, Vienna, AT, 2019, pp. 325-330.

[555] A. Vieira, B. Brogliato, And C. Prieur, Quadratic optimal control of linear complementarity systems: first order necessary conditions and numerical analysis, IEEE Transactions on Automatic Control, (2020). Extended version at https://hal.inria.fr/hal-01690400/.

[556] E. VILChES, Existence and Lyapunov pairs for the perturbed sweeping process governed by a fixed set, Set-Valued Var. Anal., 27 (2019), pp. 569-583.

[557] A. Visintin, Differential Models of Hysteresis, vol. 111 of Applied Mathematical Sciences, Springer Verlag, Berlin Heidelberg, 1994.

[558] A. Visintin, Mathematical models of hysteresis, in The Science of Hysteresis, I.-D. Mayergoyz and G. Bertotti, eds., vol. 1, Elsevier, 2005, pp. 1-123.

[559] A. Vladimirov, Equicontinuous sweeping processes, Discrete and Continuous Dynamical Systems B, 18 (2013), pp. 565-573.

[560] A.-A. Vladimirov, Nonstationary dissipative evolution equations in a Hilbert space, Nonlinear Analysis, 17 (1991), pp. 499-518.

[561] T. Vromen, N. van de Wouw, A. Doris, P. Astrid, And H. NiJmeiJer, Nonlinear outputfeedback control of torsional vibrations in drilling systems, Int. Journal of Robust and Nonlinear Control, 27 (2017), pp. 3659-3684.

[562] L.-N. Wadippuli, I. Gudoshnikov, And O. Makarenkov, Global asymptotic stability of nonconvex sweeping processes, Discrete and Continuous Dynamical Systems Series B, (2020). DoI: 10.3934/dcdsb.2019212.

[563] D.-Z.-W. WANG AND B. Du, Continuum modelling of spatial and dynamic equilibrium in a travel corridor with hererogeneous commuters-a partial differential complementarity system approach, Transportation Research Part B, 85 (2016), pp. 1-18.

[564] X. WAng AND N.-J. HuAng, Differential vector variational inequalities in finite-dimensional spaces, Journal of Optimization Theory and Applications, 158 (2013), pp. 109-129.

[565] X. WANG AND N.-J. HuAng, A class of differential vector variational inequalities in finite dimensional spaces, Journal of Optimization Theory and Applications, 162 (2014), pp. 633648.

[566] X. Wang, Y.-W. QI, C.-Q. TAO, And Y.-B. XiaO, A class of delay differential variational inequalities, Journal of Optimization Theory and Applications, 172 (2017), pp. 56-69.

[567] Y. WANG, Dynamic modeling and stability analysis of mechanical systems with time-varying topologies, ASME Journal of Mechanical Design, 115 (1993), pp. 808-816.

[568] Y. WANG, Global analysis and simulation of mechanical systems with time-varying topologies, ASME Journal of Mechanical Design, 115 (1993), pp. 817-821.

[569] C.-Z. Wu, K.-L.Teo, V. Rehbock, And G.-G. Liv, Existence and uniqueness of solutions of piecewise nonlinear systems, Nonlinear Analysis Theory Methods and Applications, 12 (2009), pp. 6109-6115.

[570] Y. Xia, H. Leung, And J. Wang, A projection neural network and its application to constrained optimization problems, IEEE Transactions on Circuits and Systems-I: Fundamental Theory and Applications, 49 (2002), pp. 447-458.

[571] Y.-S. XIA AND J. WANG, On the stability of globally projected dynamical systems, Journal of 
Optimization Theory and Applications, 106 (2000), pp. 129-150.

[572] V. YAKUBOVICH, The method of matrix inequalities in the stability theory of nonlinear control systems III. absolute stability of systems with hysteresis nonlinearities, Automation and Remote Control, 26 (1967), pp. 753-763.

[573] V.-A. YAKUBOVICH, The conditions for absolute stability of a control system with a hysteresistype nonlinearity, Soviet. Phys. Dokl., 8 (1963), pp. 235-237.

[574] V.-A. Yakubovich, Frequency conditions for the absolute stability of control systems with several nonlinear or linear nonstationary blocks, Avtomat. i Telemekh., 6 (1967), pp. 530.

[575] V.-A. Yakubovich, G.-A. Leonov, And A.-K. Gelig, Stability of Stationary Sets in Control Systems with Discontinuous Nonlinearities, vol. 14 of Stability, Vibration and Control Systems, World Scientific, 2004.

[576] Z. Yazdaniyan, M. Shamsi, Z. Foroozandeh, and M. de Pinho, A numerical method based on the complementarity and optimal control formulations for solving a family of zero-sum pursuit-evasion differential games, Journal of Computational and Applied Mathematics, (2020). paper 112535, https://doi.org/10.1016/j.cam.2019.112535.

[577] Z. Ye, Q.-G. WANG, C. Lin, C.-C. Hang, AND A.-E. Barabanov, Relay feedback analysis for a class of servo plants, Journal of Mathematical Analysis and Applications, 334 (2007), pp. $28-42$.

[578] P. YI, Y. Hong, AND F. LiU, Initialization-free distrubuted algorithms for optimal resource allocation with feasibility constraints and application to economic dispatch of power systems, Automatica, 74 (2016), pp. 259-269.

[579] K. Yoshida, M. Inoue, and T. Hatanaka, Instant MPC for linear systems and dissipativitybased stability analysis, IEEE Control Systems Letters, (2019). DOI: 10.1109/LCSYS.2019.2918095.

[580] Y. Yuan, M. Li, J. LiU, And C. Tomlin, On the powerball method: variants of descent methods for accelerated optimization, IEEE Control Systems Letters, 3 (2019), pp. 601606.

[581] G. ZAMES, On the input-output stability of nonlinear time-varying feedback systems-part I, IEEE Transactions on Automatic Control, 11 (1966), pp. 228-238.

[582] G. ZAMES, On the input-output stability of nonlinear time-varying feedback systems-part II, IEEE Transactions on Automatic Control, 11 (1966), pp. 465-477.

[583] X. Zeng, J. Chen, S. Liang, and Y. Hong, Generalized Nash equilibrium seeking strategy for distributed nonsmooth multi-cluster game, Automatica, 103 (2019), pp. 20-26.

[584] D. Zhang And A. Nagurney, On the stability of projected dynamical systems, Journal of Optimization Theory and Applications, 85 (1995), pp. 97-124.

[585] H. Zhang, J. Wei, P. YI, AND X. Hu, Projected primal-dual gradient flow of augmented Lagrangian with application to distributed maximization of the algebraic connectivity of a network, Automatica, 98 (2018), pp. 34-41.

[586] T. ZhaO, Z. LI, AND Z. Ding, Consensus-based distributed optimal energy management with less communication in a microgrid, IEEE Transactions on Industrial Informatics, 15 (2019), pp. 3356-3367.

[587] R. Zhong, F. YuAn, ANd T. PAN, A linear complementarity system approach to macroscopic freeway traffic modeling, in Proc. IEEE Int. Conference on Cyber Technology in Automation, Control and Intelligent Systems, Nanjing, China, May 2013, pp. 18-23.

[588] R.-X. Zhong, A. Sumalee, T.-L. Friesz, And W.-H.-K. Lam, Dynamic user equilibrium with side constraints for a traffic network: Theoretical development and numerical solution algorithm, Transportation Research Part B: Methodological, 45 (2011), pp. 1035-1061.

[589] R.-X. Zhong, F.-F. Yuan, T.-L. Pan, A.-H.-F. Chow, C.-J. Chen, and Z. Yang, Linear complementarity system approach to macroscopic freeway traffic modelling: uniqueness and convexity, Transportmetrica A: Transport Science, 12 (2016), pp. 142-174.

[590] V.-F. ZHURAVLEV, A method for analysing vibration-impact systems by means of special functions, Mekhanika Tverdogo Tela (Mechanics of Solids), 11 (1976), pp. 23-27.

[591] T. ZolezzI, Differential inclusions and sliding mode control, in Sliding Mode Control in Engineering, W. Perruquetti and J.-P. Barbot, eds., New York Basel, 2002, Marcel Dekker, pp. 29-52. 